Solution ${ }^{31} \mathrm{P}$ NMR study of the acid-catalyzed formation of a highly charged $\left\{U_{24} \mathrm{Pp}_{12}\right\}$ nanocluster $\left[\left(\mathrm{UO}_{2}\right)_{24}\left(\mathrm{O}_{2}\right)_{24}\left(\mathrm{P}_{2} \mathrm{O}_{7}\right)_{12}\right]^{48-}$ and its structural characterization in the solid state using single crystal neutron diffraction

Mateusz Dembowski, ${ }^{1}$ Travis A. Olds, ${ }^{2}$ Kristi L. Pellegrini, ${ }^{2}$ Christina Hoffmann, ${ }^{3}$ Xiaoping Wang, ${ }^{3}$ Sarah Hickam, ${ }^{2}$ Junhong He, ${ }^{4}$ Allen G. Oliver, ${ }^{1}$ and Peter C. Burns ${ }^{1,2}$

${ }^{1}$ Department of Chemistry and Biochemistry, University of Notre Dame, Notre Dame, Indiana 46556, United States

${ }^{2}$ Department of Civil and Environmental Engineering and Earth Sciences, University of Notre Dame, Notre Dame, Indiana 46556, United States

${ }^{3}$ Chemical and Engineering Materials Division, Oak Ridge National Laboratory, Oak Ridge, Tennessee 37831, United States

${ }^{4}$ Instrument and Source Division, Oak Ridge National Laboratory, Oak Ridge, Tennessee 37831, United States

\title{
Table of contents
}

I. Characterization of Li/Na U24Pp12
a. Raman spectroscopy
p. $S 2$
b. Infrared spectroscopy
p. S3
i. Assignment of Raman and IR peaks
p. 53
c. ICP-OES
p. 54
d. TGA
p. 54
e. ESI-MS
p. 55
f. Additional crystallographic information
p. S6-S61 


\section{Supporting information}

Raman spectroscopy measurements were performed on a Bruker Sentinel system equipped with a fiber optic probe, thermoelectric cooled CCD detector, using a $785 \mathrm{~nm}$ excitation source. Spectra of solid samples were acquired in the range from 80 to $3200 \mathrm{~cm}^{-1}$ using three $15 \mathrm{~s}$ exposures at $200 \mathrm{~mW}$ laser power on powders obtained by grinding crystalline material.

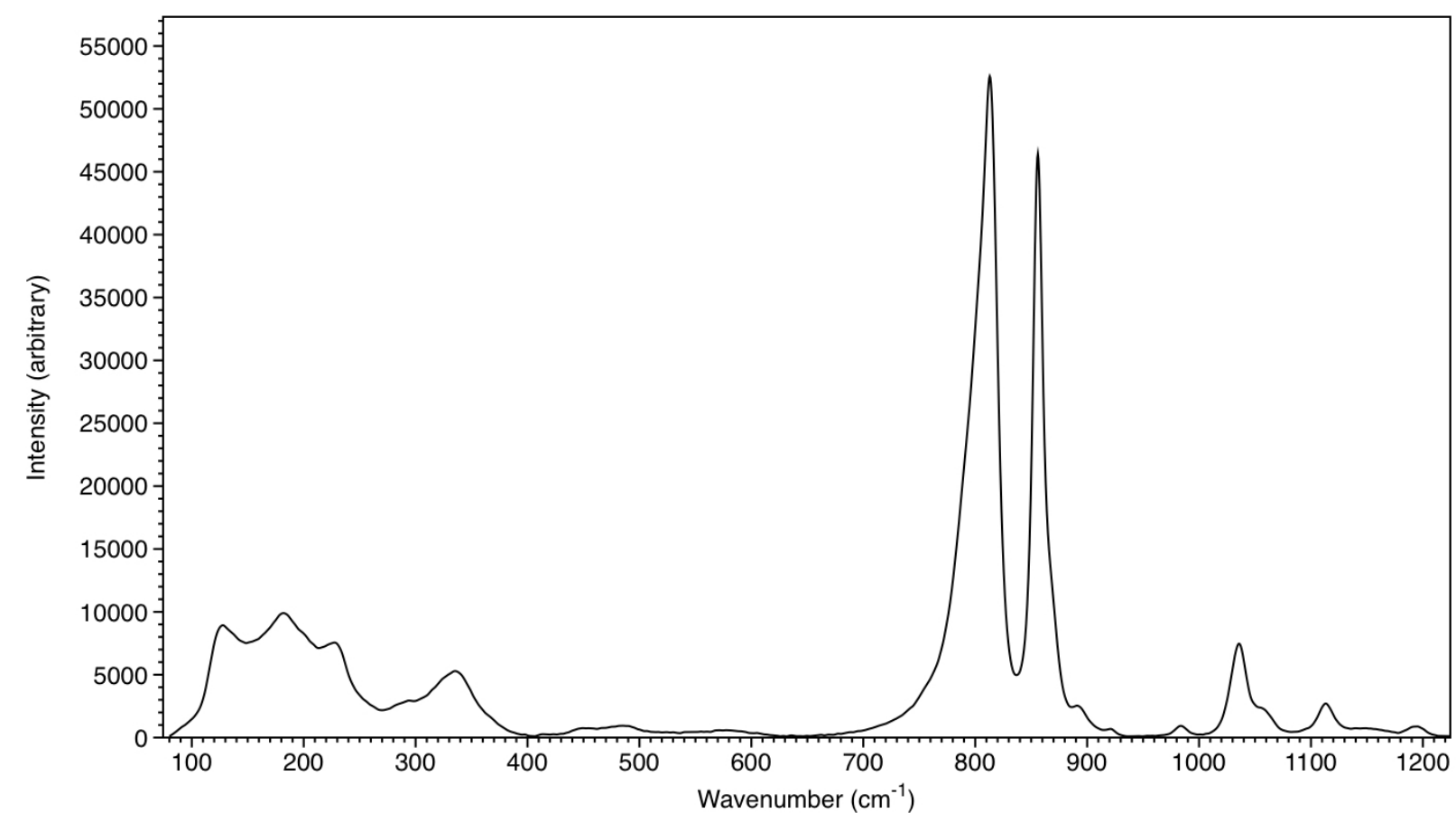

Figure S1: Raman spectrum of crystalline $\mathrm{Na} / \mathrm{K}$ salt of $\left\{\mathrm{U}_{24} \mathrm{Pp}_{12}\right\}$ 
Infrared spectroscopy measurements were performed on a SensIR Technology IlluminatIR FT-IR microspectrometer. A single crystal of $\mathbf{1}$ was placed on a glass slide, and the spectrum was collected with a diamond ATR objective in the range from 750 to $4000 \mathrm{~cm}^{-1}$.

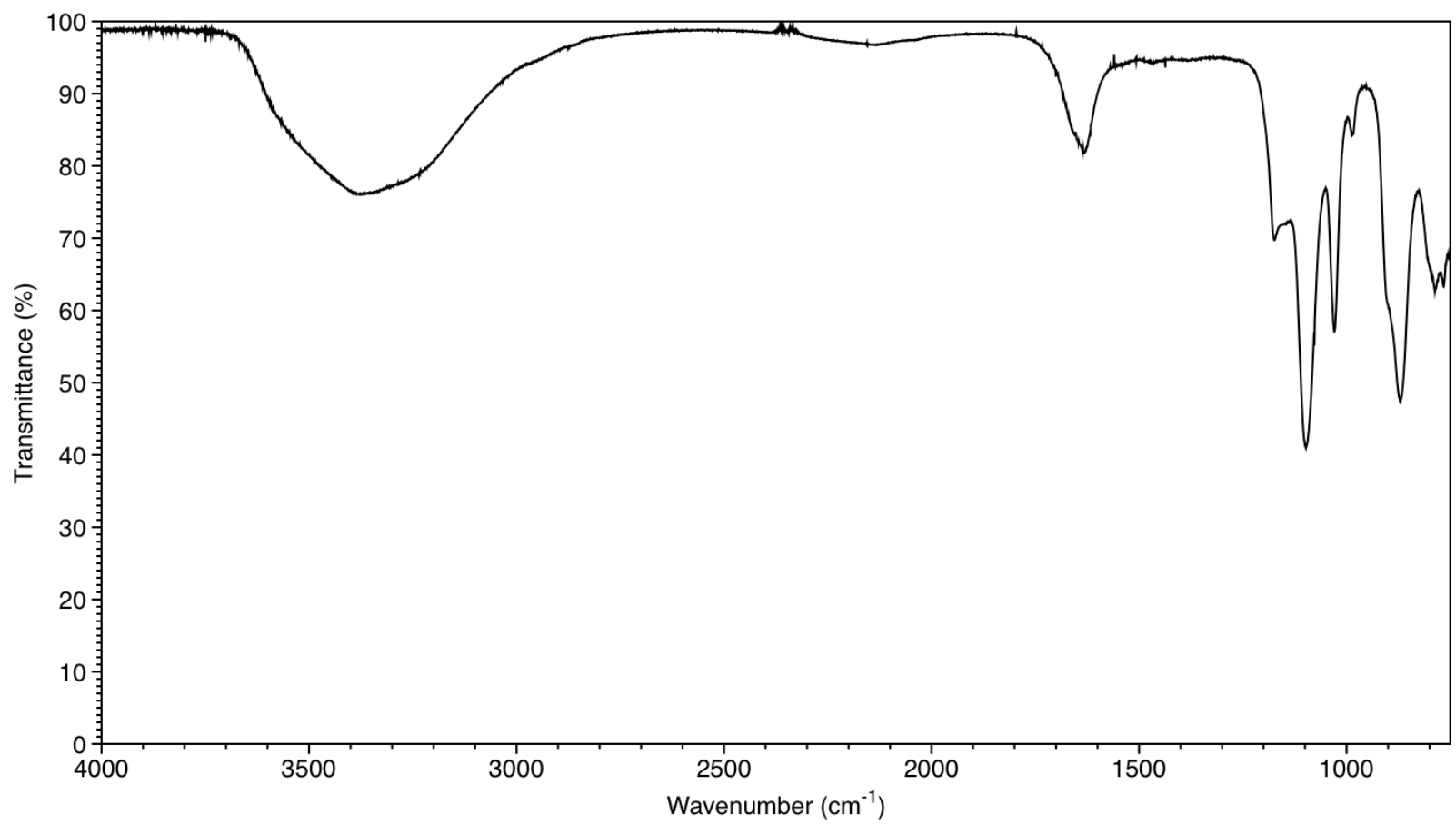

Figure S2: IR spectrum of crystalline $\mathrm{Na} / \mathrm{K}$ salt of $\left\{\mathrm{U}_{24} \mathrm{Pp}_{12}\right\}$.

Table S1: Tentative IR and Raman peak assignments

\begin{tabular}{|c|c|c|}
\hline IR & Raman & Assignment \\
\hline 3386 & & $\mathrm{H}_{2} \mathrm{O}, v_{3}$ \\
\hline \multirow[t]{2}{*}{1630} & & $\mathrm{H}_{2} \mathrm{O}, \mathrm{V}_{2}$ \\
\hline & 1195 & $\mathrm{PO}_{3}, V_{\mathrm{as}}$ \\
\hline \multirow[t]{2}{*}{1172} & & $\mathrm{PO}_{3}, V_{\text {as }}$ \\
\hline & 1114 & $\mathrm{PO}_{3}, V_{\text {as }}$ \\
\hline \multirow[t]{3}{*}{1098} & & $\mathrm{PO}, v$ \\
\hline & 1059 & $\mathrm{PO}_{3}, V_{\text {as }}$ \\
\hline & 1036 & $\mathrm{PO}_{3}, V_{\text {as }}$ \\
\hline 1026 & & $\mathrm{PO}_{3}, V_{\mathrm{as}}$ \\
\hline \multirow[t]{3}{*}{984} & 984 & $P O P, v_{a s}$ \\
\hline & 922 & $\mathrm{PO}, v$ \\
\hline & 891 & $\mathrm{PO}, v$ \\
\hline \multirow[t]{3}{*}{870} & & $-y l, v_{a s}$ \\
\hline & 856 & $\mathrm{O}_{2}, \mathrm{v}_{\mathrm{s}}$ \\
\hline & 813 & $-y l, v_{s}$ \\
\hline 787 & & $\mathrm{POP}, v_{\mathrm{s}}$ \\
\hline
\end{tabular}


Inductively Coupled Plasma Optical Emission Spectroscopy. Elemental concentrations were evaluated using a PerkinElmer Optima 8000 DV ICP-OES instrument with 165-800 nm coverage and resolution of approximately $0.01 \mathrm{~nm}$ for multielemental analysis. Samples were prepared by dilution into $5 \%$ nitric acid solutions. Standards were prepared in concentrations ranging from 0.5 to $10 \mathrm{ppm}$ for $\mathrm{Na}, \mathrm{K}$ and $\mathrm{P}$ and from 18 to $60 \mathrm{ppm}$ for U. Each standard, blank, and sample was spiked with a $0.5 \mathrm{ppm} Y$ internal standard used to monitor matrix effects.

Element concentration in ppm concentration in $\mathrm{mol} / \mathrm{L}$

\begin{tabular}{ccc}
\hline $\mathrm{Na}$ & 704 & 0.03 \\
$\mathrm{~K}$ & 7057 & 0.18 \\
\hline
\end{tabular}

Table S2: ICP-OES of 40\% TEAOH solution (Sigma-Aldrich)

Thermogravimetric Analysis measurements we performed on a Mettler Toledo TGA/DSC-1 Thermal Gravimetric Analyzer in the range from 25 to $800^{\circ} \mathrm{C}$ with $5^{\circ} \mathrm{C} / \mathrm{min}$ ramp rate. 13.5410 $\mathrm{mg}$ of crystalline $\mathrm{Na} / \mathrm{K}$ salt of $\left\{U_{24} \mathrm{Pp}_{12}\right\}$ were placed in a $70 \mu \mathrm{L}$ alumina crucible. The measurements were conducted under $\mathrm{N}_{2}$ atmosphere with $\mathrm{N}_{2}$ flow rate of $50 \mathrm{~mL} / \mathrm{min}$.

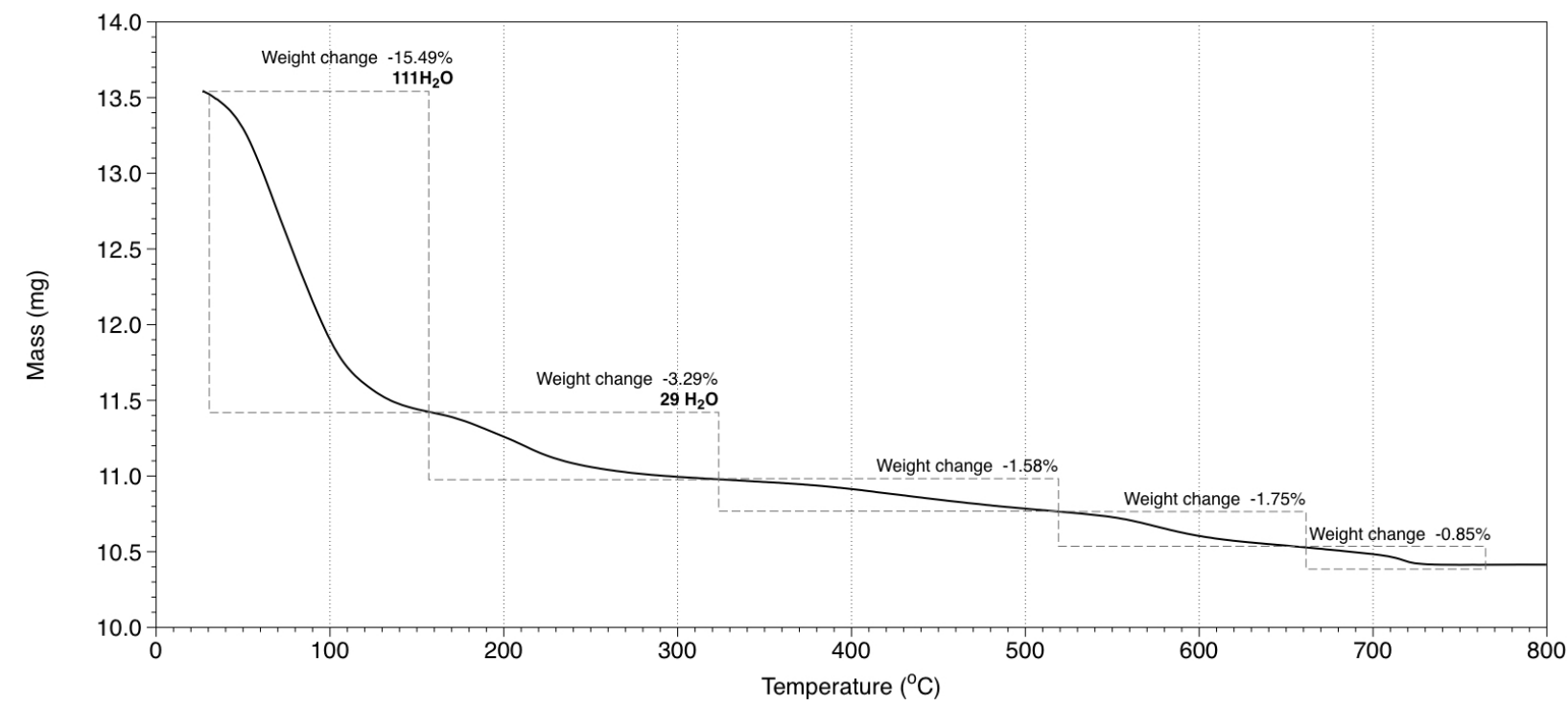

Figure S3: TGA of crystalline $\mathrm{Na} / \mathrm{K}$ salt of $\{\mathrm{U} 24 \mathrm{Pp} 12\}$ showing initial weight loss up to $157^{\circ} \mathrm{C}(-15.49 \%)$ corresponding to $111 \mathrm{H}_{2} \mathrm{O}$ molecules located in the interstitial space. Weight loss from $157^{\circ} \mathrm{C}$ to $324^{\circ} \mathrm{C}(-3.29 \%)$ corresponds to 29 internal $\mathrm{H}_{2} \mathrm{O}$ molecules. 
Electrospray Ionization-Mass Spectrometry. ESI mass spectra were acquired using a Bruker qTOF time-of-flight spectrometer. Samples were prepared to 10-20 $\mu \mathrm{M}$ concentration and directly infused into the ionization sources using syringe pump at flow rate of $4 \mu \mathrm{L} / \mathrm{min}$. TOF data were acquired in negative-ion mode at a capillary voltage of $3600 \mathrm{~V}$, and end plate offset of $-500 \mathrm{~V}, 0.8$ bar dry gas, $1.2 \mathrm{~L} / \mathrm{min}$ desolvation gas, and $180^{\circ} \mathrm{C}$ dry gas temperature.

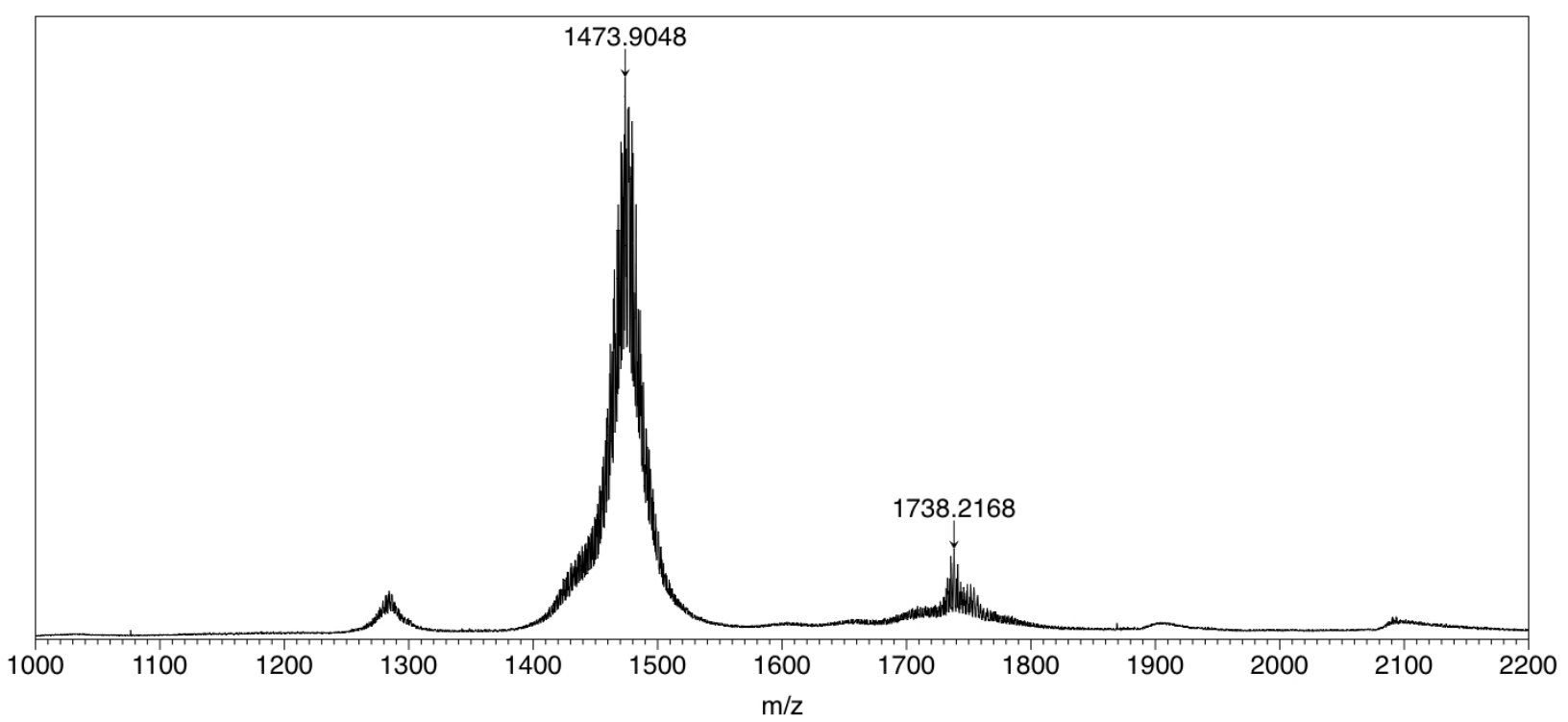

Figure S4: $\mathrm{ESI}-\mathrm{MS}$ spectrum of $\mathrm{Na} / \mathrm{K}$ salt of $\left\{\mathrm{U}_{24} \mathrm{Pp}_{12}\right\}$ in water

Table S3: ESI-MS peak deconvolution with tentative peak assignments.

\begin{tabular}{|c|c|c|c|}
\hline $\mathrm{m} / \mathbf{z}$ & charge & Formula & calculated $\mathrm{m} / \mathrm{z}$ \\
\hline \multirow[t]{9}{*}{1473.9048} & -8 & $\mathrm{~K}_{0} \mathrm{Na}_{42}\left[\left(\mathrm{UO}_{2}\right)_{24}\left(\mathrm{O}_{2}\right)_{24}\left(\mathrm{P}_{2} \mathrm{O}_{7}\right)_{12}\right]\left[\left(\left(\mathrm{O}_{3}\right)_{2}\right] \cdot \mathbf{6 2} \mathrm{H}_{2} \mathrm{O}\right.$ & 1471.0343 \\
\hline & & $\mathrm{K}_{0} \mathrm{Na}_{42}\left[\left(\mathrm{UO}_{2}\right)_{24}\left(\mathrm{O}_{2}\right)_{24}\left(\mathrm{P}_{2} \mathrm{O}_{7}\right)_{12}\right]\left[\left(\left(\mathrm{O}_{3}\right)_{2}\right] \cdot 63 \mathrm{H}_{2} \mathrm{O}\right.$ & 1473.2862 \\
\hline & & $\mathrm{K}_{0} \mathrm{Na}_{42}\left[\left(\mathrm{UO}_{2}\right)_{24}\left(\mathrm{O}_{2}\right)_{24}\left(\mathrm{P}_{2} \mathrm{O}_{7}\right)_{12}\right]\left[\left(\left(\mathrm{O}_{3}\right)_{2}\right] \cdot \mathbf{6 4} \mathrm{H}_{2} \mathrm{O}\right.$ & 1475.5381 \\
\hline & & $\mathrm{K}_{1} \mathrm{Na}_{41}\left[\left(\mathrm{UO}_{2}\right)_{24}\left(\mathrm{O}_{2}\right)_{24}\left(\mathrm{P}_{2} \mathrm{O}_{7}\right)_{12}\right]\left[\left(\left(\mathrm{O}_{3}\right)_{2}\right] \cdot \mathbf{6 1} \mathrm{H}_{2} \mathrm{O}\right.$ & 1470.7960 \\
\hline & & $\mathrm{K}_{1} \mathrm{Na}_{41}\left[\left(\mathrm{UO}_{2}\right)_{24}\left(\mathrm{O}_{2}\right)_{24}\left(\mathrm{P}_{2} \mathrm{O}_{7}\right)_{12}\right]\left[\left(\left(\mathrm{IO}_{3}\right)_{2}\right] \cdot \mathbf{6 2} \mathrm{H}_{2} \mathrm{O}\right.$ & 1473.0479 \\
\hline & & $\mathrm{K}_{1} \mathrm{Na}_{41}\left[\left(\mathrm{UO}_{2}\right)_{24}\left(\mathrm{O}_{2}\right)_{24}\left(\mathrm{P}_{2} \mathrm{O}_{7}\right)_{12}\right]\left[\left(\mathrm{IO}_{3}\right)_{2}\right] \cdot 63 \mathrm{H}_{2} \mathrm{O}$ & 1475.2998 \\
\hline & & $\mathrm{K}_{2} \mathrm{Na}_{40}\left[\left(\mathrm{UO}_{2}\right)_{24}\left(\mathrm{O}_{2}\right)_{24}\left(\mathrm{P}_{2} \mathrm{O}_{7}\right)_{12}\right]\left[\left(\left(\mathrm{O}_{3}\right)_{2}\right] \cdot \mathbf{6 1} \mathbf{H}_{2} \mathrm{O}\right.$ & 1472.8095 \\
\hline & & $\mathrm{K}_{2} \mathrm{Na}_{40}\left[\left(\mathrm{UO}_{2}\right)_{24}\left(\mathrm{O}_{2}\right)_{24}\left(\mathrm{P}_{2} \mathrm{O}_{7}\right)_{12}\right]\left[\left(\left(\mathrm{O}_{3}\right)_{2}\right] \cdot \mathbf{6 2} \mathrm{H}_{2} \mathrm{O}\right.$ & 1475.0614 \\
\hline & & $\mathrm{K}_{2} \mathrm{Na}_{40}\left[\left(\mathrm{UO}_{2}\right)_{24}\left(\mathrm{O}_{2}\right)_{24}\left(\mathrm{P}_{2} \mathrm{O}_{7}\right)_{12}\right]\left[\left(\left(\mathrm{O}_{3}\right)_{2}\right] \cdot 63 \mathrm{H}_{2} \mathrm{O}\right.$ & 1477.3134 \\
\hline \multirow[t]{5}{*}{1738.2168} & -7 & $\mathbf{K}_{0} \mathbf{N a}_{43}\left[\left(\mathrm{UO}_{2}\right)_{24}\left(\mathrm{O}_{2}\right)_{24}\left(\mathrm{P}_{2} \mathrm{O}_{7}\right)_{12}\right]\left[\left(\left(\mathrm{O}_{3}\right)_{2}\right] \cdot \mathbf{8 2} \mathbf{H}_{2} \mathrm{O}\right.$ & 1735.9385 \\
\hline & & $\mathrm{K}_{0} \mathrm{Na}_{43}\left[\left(\mathrm{UO}_{2}\right)_{24}\left(\mathrm{O}_{2}\right)_{24}\left(\mathrm{P}_{2} \mathrm{O}_{7}\right)_{12}\right]\left[\left(\left(\mathrm{IO}_{3}\right)_{2}\right] \cdot \mathbf{8 3} \mathrm{H}_{2} \mathrm{O}\right.$ & 1738.5121 \\
\hline & & $\mathbf{K}_{1} \mathbf{N a}_{42}\left[\left(\mathrm{UO}_{2}\right)_{24}\left(\mathrm{O}_{2}\right)_{24}\left(\mathrm{P}_{2} \mathrm{O}_{7}\right)_{12}\right]\left[\left(\left(\mathrm{O}_{3}\right)_{2}\right] \cdot \mathbf{8 1} \mathbf{H}_{2} \mathbf{O}\right.$ & 1735.6661 \\
\hline & & $\mathrm{K}_{1} \mathrm{Na}_{42}\left[\left(\mathrm{UO}_{2}\right)_{24}\left(\mathrm{O}_{2}\right)_{24}\left(\mathrm{P}_{2} \mathrm{O}_{7}\right)_{12}\right]\left[\left(\left(\mathrm{O}_{3}\right)_{2}\right] \cdot \mathbf{8 2} \mathbf{H}_{\mathbf{2}} \mathrm{O}\right.$ & 1738.2398 \\
\hline & & $\mathrm{K}_{1} \mathrm{Na}_{42}\left[\left(\mathrm{UO}_{2}\right)_{24}\left(\mathrm{O}_{2}\right)_{24}\left(\mathrm{P}_{2} \mathrm{O}_{7}\right)_{12}\right]\left[\left(\left(\mathrm{O}_{3}\right)_{2}\right] \cdot \mathbf{8 3} \mathrm{H}_{2} \mathrm{O}\right.$ & 1740.8134 \\
\hline
\end{tabular}


Table S4: Crystal data and structure refinement for $\mathrm{Na}_{44} \mathrm{~K}_{6}\left[\left(\mathrm{UO}_{2}\right)_{24}\left(\mathrm{O}_{2}\right)_{24}\left(\mathrm{P}_{2} \mathrm{O}_{7}\right)_{12}\right]\left[1 \mathrm{IO}_{3}\right]_{2} \cdot 140 \mathrm{H}_{2} \mathrm{O}$

\begin{tabular}{|c|c|c|}
\hline Identification code & \multicolumn{2}{|l|}{$\mathrm{Na} / \mathrm{K}\{\mathrm{U} 24 \mathrm{Pp} 12\}$} \\
\hline Empirical formula & \multicolumn{2}{|c|}{$\mathrm{H}_{120.75} \mathrm{IK}_{3} \mathrm{Na}_{19.50} \mathrm{O}_{167.25} \mathrm{P}_{12} \mathrm{U}_{12}$} \\
\hline Formula weight & \multicolumn{2}{|l|}{6718.21} \\
\hline Temperature & \multicolumn{2}{|l|}{$100(2) \mathrm{K}$} \\
\hline Wavelength & \multicolumn{2}{|l|}{$0.40-3.40 \AA$} \\
\hline Crystal system & \multicolumn{2}{|l|}{ Triclinic } \\
\hline Space group & \multicolumn{2}{|l|}{$\mathrm{P}-1$} \\
\hline \multirow[t]{3}{*}{ Unit cell dimensions } & $a=21.886(4) \AA$ & $\alpha=62.227(2)^{\circ}$ \\
\hline & $b=22.379(4) \AA$ & $\beta=87.715(2)^{\circ}$ \\
\hline & $c=22.717(4) \AA$ & $\gamma=61.278(2)^{\circ}$ \\
\hline Volume & \multicolumn{2}{|l|}{$8387(2) \AA^{3}$} \\
\hline Z & \multicolumn{2}{|l|}{2} \\
\hline Density (calculated) & \multicolumn{2}{|l|}{$2.660 \mathrm{~g} \cdot \mathrm{cm}^{-3}$} \\
\hline Absorption coefficient $(\mu)$ & \multicolumn{2}{|l|}{$0.2172 \mathrm{~mm}^{-1}$} \\
\hline$F(000)$ & \multicolumn{2}{|l|}{6134} \\
\hline Crystal size & \multicolumn{2}{|c|}{$1.5 \times 1.25 \times 0.75 \mathrm{~mm}^{3}$} \\
\hline$\theta$ range for data collection & \multicolumn{2}{|l|}{7.462 to $78.554^{\circ}$} \\
\hline Index ranges & \multicolumn{2}{|c|}{$-28 \leq h \leq 29,-29 \leq k \leq 29,-30 \leq 1 \leq 30$} \\
\hline Reflections collected & \multicolumn{2}{|l|}{31629} \\
\hline Independent reflections & \multicolumn{2}{|c|}{$14914\left[\mathrm{R}_{\mathrm{int}}=0.1111\right]$} \\
\hline Completeness to $\theta=25.242^{\circ}$ & \multicolumn{2}{|l|}{$42.7 \%$} \\
\hline Absorption correction & \multicolumn{2}{|l|}{ Sphere } \\
\hline Max. and min. transmission & \multicolumn{2}{|c|}{0.8364 and 0.5933} \\
\hline Refinement method & \multicolumn{2}{|c|}{ Full-matrix least-squares on $\mathrm{F}^{2}$} \\
\hline Data / restraints / parameters & \multicolumn{2}{|c|}{14914 / 100 / 2633} \\
\hline Goodness-of-fit on $\mathrm{F}^{2}$ & \multicolumn{2}{|l|}{1.100} \\
\hline Final $R$ indices $[1>2 \sigma(I)]$ & \multicolumn{2}{|c|}{$R_{1}=0.1061, w R_{2}=0.2625$} \\
\hline $\mathrm{R}$ indices (all data) & \multicolumn{2}{|c|}{$R_{1}=0.1063, w R_{2}=0.2626$} \\
\hline Extinction coefficient & \multicolumn{2}{|l|}{$\mathrm{n} / \mathrm{a}$} \\
\hline Largest diff. peak and hole & \multicolumn{2}{|c|}{4.082 and $-2.908 e^{-} . \AA^{-3}$} \\
\hline
\end{tabular}


Table S5: Atomic coordinates and equivalent isotropic displacement parameters $\left(\AA^{2}\right)$ for $\mathrm{Na} / \mathrm{K}\left\{\mathrm{U}_{24} \mathrm{Pp}_{12}\right\}$. $\mathrm{U}(\mathrm{eq})$ is defined as one third of the trace of the orthogonalized $\mathrm{U}_{\mathrm{ij}}$ tensor.

\begin{tabular}{|c|c|c|c|c|}
\hline & $x$ & $y$ & $z$ & $U(e q)$ \\
\hline$U(1)$ & $1.3963(3)$ & $1.1024(3)$ & $-0.3044(2)$ & $0.011(1)$ \\
\hline$U(2)$ & $1.0012(3)$ & $1.6993(3)$ & $-0.2756(2)$ & $0.011(1)$ \\
\hline$U(3)$ & $0.6982(3)$ & $1.6505(3)$ & $-0.4480(2)$ & $0.010(1)$ \\
\hline$U(4)$ & $0.8497(3)$ & $1.5810(3)$ & $-0.2842(2)$ & $0.011(1)$ \\
\hline$U(5)$ & $0.7798(3)$ & $1.4097(3)$ & $-0.3786(2)$ & $0.010(1)$ \\
\hline$U(6)$ & $1.2466(3)$ & $1.0309(3)$ & $-0.2683(3)$ & $0.012(1)$ \\
\hline$U(7)$ & $0.9335(3)$ & $1.3414(3)$ & $-0.2205(2)$ & $0.011(1)$ \\
\hline$U(8)$ & $1.2112(3)$ & $1.5441(3)$ & $-0.2660(2)$ & $0.011(1)$ \\
\hline$U(9)$ & $1.0212(3)$ & $1.1435(3)$ & $-0.5174(2)$ & $0.011(1)$ \\
\hline$U(10)$ & $1.1944(3)$ & $1.7062(3)$ & $-0.4655(3)$ & $0.012(1)$ \\
\hline$U(11)$ & $1.3307(3)$ & $1.1855(3)$ & $-0.1678(3)$ & $0.012(1)$ \\
\hline$U(12)$ & $1.1946(3)$ & $1.0979(3)$ & $-0.1209(3)$ & $0.012(1)$ \\
\hline $\mathrm{I}(1)$ & $0.8142(5)$ & $1.4309(6)$ & $-0.0992(4)$ & $0.023(2)$ \\
\hline $\mathrm{K}(1)$ & $1.0559(8)$ & $1.4844(7)$ & $-0.2274(7)$ & $0.025(3)$ \\
\hline$K(2)$ & $1.2704(8)$ & $1.4860(7)$ & $-0.4210(7)$ & $0.025(3)$ \\
\hline$K(3)$ & $1.0440(8)$ & $1.1779(11)$ & $-0.2700(8)$ & $0.035(4)$ \\
\hline $\mathrm{Na}(1)$ & $1.2296(7)$ & $1.3249(7)$ & $-0.3752(7)$ & $0.019(3)$ \\
\hline $\mathrm{Na}(2)$ & $1.0257(7)$ & $1.8142(7)$ & $-0.1918(6)$ & $0.018(3)$ \\
\hline $\mathrm{Na}(3)$ & $0.7183(7)$ & $1.4966(8)$ & $-0.2558(7)$ & $0.022(3)$ \\
\hline $\mathrm{Na}(4)$ & $0.4205(6)$ & $2.0447(7)$ & $-0.5568(6)$ & $0.014(2)$ \\
\hline $\mathrm{Na}(5)$ & $0.8609(7)$ & $1.5127(7)$ & $-0.0078(7)$ & $0.021(3)$ \\
\hline $\mathrm{Na}(6)$ & $0.9621(7)$ & $1.5914(7)$ & $0.0008(6)$ & $0.019(3)$ \\
\hline $\mathrm{Na}(7)$ & $1.1223(7)$ & $1.3234(7)$ & $-0.2678(7)$ & $0.021(3)$ \\
\hline $\mathrm{Na}(8)$ & $0.9227(7)$ & $1.0699(7)$ & $0.0127(6)$ & $0.018(3)$ \\
\hline $\mathrm{Na}(9)$ & $0.8815(7)$ & $1.9903(8)$ & $-0.3254(7)$ & $0.020(3)$ \\
\hline $\mathrm{Na}(10)$ & $1.5005(8)$ & $1.5783(8)$ & $-0.5782(7)$ & $0.024(3)$ \\
\hline $\mathrm{Na}(11)$ & $0.9411(8)$ & $1.6838(9)$ & $-0.4264(8)$ & $0.025(3)$ \\
\hline $\mathrm{Na}(12)$ & $1.1798(8)$ & $0.8246(8)$ & $-0.2817(7)$ & $0.023(3)$ \\
\hline $\mathrm{Na}(13)$ & $1.2582(8)$ & $1.8583(8)$ & $-0.5207(7)$ & $0.025(3)$ \\
\hline $\mathrm{Na}(14)$ & $0.4826(10)$ & $1.8685(9)$ & $-0.3931(8)$ & $0.033(4)$ \\
\hline $\mathrm{Na}(15)$ & $1.4676(8)$ & $1.5941(8)$ & $-0.4347(7)$ & $0.022(3)$ \\
\hline
\end{tabular}




\begin{tabular}{|c|c|c|c|c|}
\hline $\mathrm{Na}(16)$ & 1.5000 & 1.0000 & 0.0000 & $0.053(8)$ \\
\hline $\mathrm{Na}(17)$ & $0.9244(14)$ & $1.0591(11)$ & $-0.1354(10)$ & $0.054(5)$ \\
\hline $\mathrm{Na}(18)$ & $1.4085(13)$ & $1.7233(15)$ & $-0.3891(12)$ & $0.062(6)$ \\
\hline $\mathrm{Na}(19)$ & $0.7280(13)$ & $1.8106(13)$ & $-0.4662(12)$ & $0.056(5)$ \\
\hline $\mathrm{Na}(20)$ & $0.6758(16)$ & $1.6206(17)$ & $-0.0923(15)$ & $0.051(6)$ \\
\hline $\mathrm{Na}(21)$ & $0.655(4)$ & $1.575(4)$ & $-0.053(3)$ & $0.033(14)$ \\
\hline$P(1)$ & $0.8193(4)$ & $1.7392(5)$ & $-0.2585(4)$ & $0.012(2)$ \\
\hline$P(2)$ & $1.3411(4)$ & $1.6038(5)$ & $-0.5297(4)$ & $0.013(2)$ \\
\hline$P(3)$ & $1.2665(5)$ & $1.3473(5)$ & $-0.1291(5)$ & $0.019(2)$ \\
\hline$P(4)$ & $1.4697(4)$ & $1.2165(5)$ & $-0.4264(4)$ & $0.012(2)$ \\
\hline$P(5)$ & $1.1085(5)$ & $1.0152(5)$ & $-0.3322(4)$ & $0.013(2)$ \\
\hline$P(6)$ & $1.3597(4)$ & $1.3516(5)$ & $-0.2181(4)$ & $0.013(2)$ \\
\hline$P(7)$ & $0.9275(4)$ & $1.5894(5)$ & $-0.1485(4)$ & $0.012(2)$ \\
\hline$P(8)$ & $1.2286(5)$ & $1.7542(5)$ & $-0.6375(4)$ & $0.014(2)$ \\
\hline$P(9)$ & $1.0571(5)$ & $1.2065(5)$ & $-0.0532(4)$ & $0.015(2)$ \\
\hline$P(10)$ & $1.2074(4)$ & $1.0231(4)$ & $-0.4154(4)$ & $0.012(2)$ \\
\hline$P(11)$ & $0.5639(4)$ & $1.8643(5)$ & $-0.5255(4)$ & $0.013(2)$ \\
\hline$P(12)$ & $1.0168(5)$ & $1.1294(5)$ & $-0.1002(4)$ & $0.013(2)$ \\
\hline $\mathrm{O}(1)$ & $1.3572(4)$ & $1.0158(5)$ & $-0.2424(4)$ & $0.017(2)$ \\
\hline$O(2)$ & $1.4122(4)$ & $1.1504(4)$ & $-0.2355(4)$ & $0.015(2)$ \\
\hline$O(3)$ & $1.3050(4)$ & $1.1844(4)$ & $-0.3408(4)$ & $0.016(2)$ \\
\hline $\mathrm{O}(4)$ & $1.3972(4)$ & $1.0879(4)$ & $-0.1951(4)$ & $0.016(2)$ \\
\hline$O(5)$ & $1.4876(4)$ & $1.0207(5)$ & $-0.2702(4)$ & $0.019(2)$ \\
\hline$O(6)$ & $1.4346(4)$ & $1.1934(4)$ & $-0.3679(4)$ & $0.016(2)$ \\
\hline $\mathrm{O}(7)$ & $0.5875(4)$ & $1.9024(5)$ & $-0.5902(4)$ & $0.018(2)$ \\
\hline $\mathrm{O}(8)$ & $1.3497(4)$ & $1.0263(5)$ & $-0.3107(4)$ & $0.017(2)$ \\
\hline $\mathrm{O}(9)$ & $0.8762(4)$ & $1.7464(4)$ & $-0.2944(4)$ & $0.014(1)$ \\
\hline $\mathrm{O}(10)$ & $1.0134(4)$ & $1.8003(4)$ & $-0.3641(4)$ & $0.015(2)$ \\
\hline $\mathrm{O}(11)$ & $0.9883(4)$ & $1.5994(4)$ & $-0.1772(4)$ & $0.016(2)$ \\
\hline $\mathrm{O}(12)$ & $1.0093(4)$ & $1.6528(4)$ & $-0.3243(4)$ & $0.013(1)$ \\
\hline $\mathrm{O}(13)$ & $1.1232(4)$ & $1.6547(4)$ & $-0.2607(4)$ & $0.015(2)$ \\
\hline $\mathrm{O}(14)$ & $0.9918(4)$ & $1.7435(4)$ & $-0.2245(4)$ & $0.017(2)$ \\
\hline $\mathrm{O}(15)$ & $1.1181(4)$ & $1.5878(4)$ & $-0.2077(4)$ & $0.018(2)$ \\
\hline $\mathrm{O}(16)$ & $0.7794(4)$ & $1.5268(4)$ & $-0.4307(4)$ & $0.013(1)$ \\
\hline $\mathrm{O}(17)$ & $0.7644(4)$ & $1.6781(4)$ & $-0.3924(4)$ & $0.014(2)$ \\
\hline $\mathrm{O}(18)$ & $0.8101(4)$ & $1.6014(4)$ & $-0.3881(4)$ & $0.012(1)$ \\
\hline
\end{tabular}




\begin{tabular}{|c|c|c|c|c|}
\hline $\mathrm{O}(19)$ & $0.7106(4)$ & $1.5482(4)$ & $-0.4648(4)$ & $0.014(2)$ \\
\hline $\mathrm{O}(20)$ & $1.4173(4)$ & $1.2973(4)$ & $-0.4891(4)$ & $0.015(2)$ \\
\hline $\mathrm{O}(21)$ & $0.6259(4)$ & $1.7837(4)$ & $-0.4725(4)$ & $0.016(2)$ \\
\hline$O(22)$ & $0.6690(4)$ & $1.6134(5)$ & $-0.3694(4)$ & $0.016(2)$ \\
\hline$O(23)$ & $0.9068(5)$ & $1.5509(4)$ & $-0.1780(4)$ & $0.018(2)$ \\
\hline$O(24)$ & $0.9132(4)$ & $1.4680(4)$ & $-0.2912(4)$ & $0.013(1)$ \\
\hline$O(25)$ & $0.9148(4)$ & $1.6048(4)$ & $-0.3210(4)$ & $0.014(1)$ \\
\hline$O(26)$ & $0.8851(4)$ & $1.3912(4)$ & $-0.3333(4)$ & $0.015(2)$ \\
\hline $\mathrm{O}(27)$ & $1.3279(4)$ & $1.5646(4)$ & $-0.5641(4)$ & $0.014(2)$ \\
\hline $\mathrm{O}(28)$ & $1.2159(4)$ & $1.7125(4)$ & $-0.6686(4)$ & $0.015(2)$ \\
\hline$O(29)$ & $0.8218(4)$ & $1.3926(4)$ & $-0.4426(4)$ & $0.015(2)$ \\
\hline$O(30)$ & $0.8925(4)$ & $1.3130(4)$ & $-0.2942(4)$ & $0.014(2)$ \\
\hline $\mathrm{O}(31)$ & $1.2232(4)$ & $1.0627(5)$ & $-0.3846(4)$ & $0.018(2)$ \\
\hline $\mathrm{O}(32)$ & $1.1348(4)$ & $1.0385(5)$ & $-0.2900(4)$ & $0.017(2)$ \\
\hline $\mathrm{O}(33)$ & $1.2668(4)$ & $1.0123(5)$ & $-0.1597(4)$ & $0.016(2)$ \\
\hline $\mathrm{O}(34)$ & $0.9925(4)$ & $1.2876(4)$ & $-0.1054(4)$ & $0.017(2)$ \\
\hline $\mathrm{O}(35)$ & $1.2663(4)$ & $1.5940(4)$ & $-0.3545(4)$ & $0.014(2)$ \\
\hline $\mathrm{O}(36)$ & $1.0200(4)$ & $1.3018(4)$ & $-0.2407(4)$ & $0.016(2)$ \\
\hline $\mathrm{O}(37)$ & $0.9531(4)$ & $1.2108(4)$ & $-0.1538(4)$ & $0.016(2)$ \\
\hline $\mathrm{O}(38)$ & $0.8482(4)$ & $1.3795(4)$ & $-0.1997(4)$ & $0.016(2)$ \\
\hline $\mathrm{O}(39)$ & $1.2085(4)$ & $1.6583(4)$ & $-0.3475(4)$ & $0.015(2)$ \\
\hline $\mathrm{O}(40)$ & $1.2751(4)$ & $1.5279(4)$ & $-0.2036(4)$ & $0.017(2)$ \\
\hline $\mathrm{O}(41)$ & $1.1502(4)$ & $1.5529(5)$ & $-0.3256(4)$ & $0.020(2)$ \\
\hline $\mathrm{O}(42)$ & $0.9372(4)$ & $1.8262(4)$ & $-0.3796(4)$ & $0.014(2)$ \\
\hline $\mathrm{O}(43)$ & $0.7263(4)$ & $1.6892(4)$ & $-0.5258(4)$ & $0.016(2)$ \\
\hline $\mathrm{O}(44)$ & $0.7888(4)$ & $1.7015(4)$ & $-0.2796(4)$ & $0.016(2)$ \\
\hline $\mathrm{O}(45)$ & $0.7848(4)$ & $1.5564(4)$ & $-0.2456(4)$ & $0.016(2)$ \\
\hline $\mathrm{O}(46)$ & $0.7344(4)$ & $1.4285(4)$ & $-0.3154(4)$ & $0.015(2)$ \\
\hline $\mathrm{O}(47)$ & $1.1967(4)$ & $1.1373(4)$ & $-0.3068(4)$ & $0.016(2)$ \\
\hline $\mathrm{O}(48)$ & $1.2921(4)$ & $0.9250(4)$ & $-0.2334(4)$ & $0.017(2)$ \\
\hline $\mathrm{O}(49)$ & $1.1965(4)$ & $1.0221(5)$ & $-0.1701(4)$ & $0.017(2)$ \\
\hline $\mathrm{O}(50)$ & $1.2064(5)$ & $1.4298(5)$ & $-0.1835(5)$ & $0.029(2)$ \\
\hline $\mathrm{O}(51)$ & $1.0497(4)$ & $1.0896(5)$ & $-0.3954(4)$ & $0.020(2)$ \\
\hline $\mathrm{O}(52)$ & $1.1004(4)$ & $1.8009(4)$ & $-0.4463(4)$ & $0.013(1)$ \\
\hline $\mathrm{O}(53)$ & $1.1491(4)$ & $1.0831(4)$ & $-0.4824(4)$ & $0.016(2)$ \\
\hline$O(54)$ & $1.0078(4)$ & $1.2336(4)$ & $-0.5271(4)$ & $0.015(2)$ \\
\hline
\end{tabular}




\begin{tabular}{|c|c|c|c|c|}
\hline $\mathrm{O}(55)$ & $1.0866(4)$ & $1.8357(4)$ & $-0.5212(4)$ & $0.015(2)$ \\
\hline $\mathrm{O}(56)$ & $1.0389(4)$ & $1.0501(4)$ & $-0.5048(4)$ & $0.013(1)$ \\
\hline $\mathrm{O}(57)$ & $1.2951(4)$ & $1.6093(4)$ & $-0.4784(4)$ & $0.017(2)$ \\
\hline $\mathrm{O}(58)$ & $1.1801(4)$ & $1.7666(4)$ & $-0.5885(4)$ & $0.015(2)$ \\
\hline $\mathrm{O}(59)$ & $1.1444(4)$ & $1.6620(4)$ & $-0.4647(4)$ & $0.015(2)$ \\
\hline $\mathrm{O}(60)$ & $1.2468(4)$ & $1.7484(4)$ & $-0.4661(4)$ & $0.017(2)$ \\
\hline$O(61)$ & $1.2577(4)$ & $1.2406(4)$ & $-0.2404(4)$ & $0.019(2)$ \\
\hline$O(62)$ & $1.2635(5)$ & $1.2839(5)$ & $-0.1352(4)$ & $0.020(2)$ \\
\hline$O(63)$ & $1.3082(4)$ & $1.0826(5)$ & $-0.1113(4)$ & $0.018(2)$ \\
\hline$O(64)$ & $1.2622(4)$ & $1.1430(5)$ & $-0.0935(4)$ & $0.018(2)$ \\
\hline$O(65)$ & $1.3553(5)$ & $1.2894(5)$ & $-0.2265(4)$ & $0.025(2)$ \\
\hline$O(66)$ & $1.1623(4)$ & $1.1801(4)$ & $-0.2057(4)$ & $0.016(2)$ \\
\hline $\mathrm{O}(67)$ & $1.1227(4)$ & $1.1869(5)$ & $-0.0825(4)$ & $0.017(2)$ \\
\hline $\mathrm{O}(68)$ & $1.0830(4)$ & $1.1065(5)$ & $-0.1285(4)$ & $0.019(2)$ \\
\hline O(69) & $1.2255(4)$ & $1.0140(5)$ & $-0.0333(4)$ & $0.019(2)$ \\
\hline $\mathrm{O}(70)$ & $0.7617(4)$ & $1.8152(5)$ & $-0.2631(4)$ & $0.021(2)$ \\
\hline $\mathrm{O}(71)$ & $0.8585(4)$ & $1.6774(4)$ & $-0.1774(4)$ & $0.014(2)$ \\
\hline $\mathrm{O}(72)$ & $1.4183(4)$ & $1.5708(4)$ & $-0.5047(4)$ & $0.013(1)$ \\
\hline $\mathrm{O}(73)$ & $1.3100(4)$ & $1.6950(4)$ & $-0.5907(4)$ & $0.013(1)$ \\
\hline $\mathrm{O}(74)$ & $0.5071(4)$ & $1.8474(4)$ & $-0.5496(4)$ & $0.013(1)$ \\
\hline $\mathrm{O}(75)$ & $1.5390(4)$ & $1.2090(4)$ & $-0.4063(4)$ & $0.017(2)$ \\
\hline $\mathrm{O}(76)$ & $0.9406(4)$ & $1.5492(4)$ & $-0.0726(4)$ & $0.014(2)$ \\
\hline $\mathrm{O}(77)$ & $0.5228(4)$ & $1.9169(4)$ & $-0.4975(4)$ & $0.014(1)$ \\
\hline $\mathrm{O}(78)$ & $1.0694(4)$ & $1.1980(4)$ & $0.0158(4)$ & $0.017(2)$ \\
\hline $\mathrm{O}(79)$ & $1.0360(4)$ & $1.1422(4)$ & $-0.0406(4)$ & $0.016(2)$ \\
\hline $\mathrm{O}(80)$ & $0.9971(4)$ & $1.0683(4)$ & $-0.0678(4)$ & $0.016(2)$ \\
\hline $\mathrm{O}(81)$ & $1.2272(5)$ & $1.8272(4)$ & $-0.6907(4)$ & $0.017(2)$ \\
\hline $\mathrm{O}(82)$ & $1.1756(4)$ & $0.9739(4)$ & $-0.3618(4)$ & $0.016(2)$ \\
\hline $\mathrm{O}(83)$ & $1.2742(4)$ & $0.9630(4)$ & $-0.4226(4)$ & $0.015(2)$ \\
\hline $\mathrm{O}(84)$ & $1.3410(4)$ & $1.3402(5)$ & $-0.1458(4)$ & $0.020(2)$ \\
\hline $\mathrm{O}(85)$ & $0.9438(4)$ & $1.4444(4)$ & $-0.2217(4)$ & $0.016(2)$ \\
\hline $\mathrm{O}(86)$ & $1.3010(5)$ & $1.4329(5)$ & $-0.2730(5)$ & $0.028(2)$ \\
\hline $\mathrm{O}(87)$ & $1.4044(4)$ & $1.1339(4)$ & $-0.0957(4)$ & $0.017(2)$ \\
\hline $\mathrm{O}(88)$ & $1.2695(7)$ & $1.3352(7)$ & $-0.0586(5)$ & $0.039(3)$ \\
\hline $\mathrm{O}(89)$ & $1.0902(5)$ & $0.9535(5)$ & $-0.2926(4)$ & $0.021(2)$ \\
\hline $\mathrm{O}(90)$ & $1.4321(6)$ & $1.3385(8)$ & $-0.2149(6)$ & $0.041(3)$ \\
\hline
\end{tabular}




\begin{tabular}{|c|c|c|c|c|}
\hline $\mathrm{O}(1 \mathrm{I})$ & $0.8374(6)$ & $1.3378(5)$ & $-0.0245(5)$ & $0.029(2)$ \\
\hline $\mathrm{O}(2 \mathrm{I})$ & $0.7903(5)$ & $1.4946(6)$ & $-0.0644(5)$ & $0.031(2)$ \\
\hline $\mathrm{O}(3 \mathrm{I})$ & $0.7271(5)$ & $1.4592(5)$ & $-0.1375(5)$ & $0.026(2)$ \\
\hline $\mathrm{O}(\mathrm{OW})$ & $1.1179(6)$ & $0.8829(6)$ & $-0.3919(5)$ & $0.029(2)$ \\
\hline $\mathrm{O}(1 \mathrm{~W})$ & $1.1434(5)$ & $0.7371(6)$ & $-0.2063(5)$ & $0.026(2)$ \\
\hline $\mathrm{O}(2 \mathrm{~W})$ & $1.2640(6)$ & $0.7209(6)$ & $-0.3007(6)$ & $0.033(2)$ \\
\hline $\mathrm{O}(3 \mathrm{~W})$ & $1.2700(8)$ & $0.8011(7)$ & $-0.2076(6)$ & $0.045(3)$ \\
\hline $\mathrm{O}(4 \mathrm{~W})$ & $1.1245(4)$ & $1.3265(4)$ & $-0.1672(4)$ & $0.017(2)$ \\
\hline$O(5 W)$ & $1.3343(4)$ & $1.3278(5)$ & $-0.3610(4)$ & $0.017(2)$ \\
\hline $\mathrm{O}(6 \mathrm{~W})$ & $0.8347(5)$ & $1.7925(5)$ & $-0.4320(5)$ & $0.024(2)$ \\
\hline$O(7 W)$ & $0.9588(6)$ & $1.2177(5)$ & $-0.3790(5)$ & $0.034(3)$ \\
\hline $\mathrm{O}(8 \mathrm{~W})$ & $1.5555(5)$ & $1.5899(5)$ & $-0.5020(4)$ & $0.021(2)$ \\
\hline $\mathrm{O}(9 \mathrm{~W})$ & $1.0409(5)$ & $1.6150(5)$ & $-0.0687(4)$ & $0.023(2)$ \\
\hline$O(10 W)$ & $1.0379(5)$ & $1.7426(5)$ & $-0.0745(4)$ & $0.021(2)$ \\
\hline$O(11 W)$ & $1.1289(5)$ & $1.9428(5)$ & $-0.5524(4)$ & $0.020(2)$ \\
\hline $\mathrm{O}(12 \mathrm{~W})$ & $1.1334(5)$ & $1.2994(5)$ & $-0.3615(4)$ & $0.020(2)$ \\
\hline$O(13 W)$ & $1.2419(4)$ & $1.9100(5)$ & $-0.6414(5)$ & $0.023(2)$ \\
\hline$O(14 W)$ & $1.0010(5)$ & $1.8990(5)$ & $-0.3137(4)$ & $0.020(2)$ \\
\hline$O(15 W)$ & $0.8917(5)$ & $1.5498(5)$ & $0.0723(5)$ & $0.025(2)$ \\
\hline$O(16 W)$ & $0.5220(4)$ & $1.9265(5)$ & $-0.3506(5)$ & $0.022(2)$ \\
\hline$O(17 W)$ & $1.5213(5)$ & $1.6422(7)$ & $-0.3910(5)$ & $0.034(2)$ \\
\hline $\mathrm{O}(18 \mathrm{~W})$ & $0.3428(5)$ & $2.0383(5)$ & $-0.6219(5)$ & $0.023(2)$ \\
\hline$O(19 W)$ & $0.8366(5)$ & $1.9184(5)$ & $-0.3403(5)$ & $0.025(2)$ \\
\hline$O(20 W)$ & $0.3384(6)$ & $2.1836(5)$ & $-0.6219(5)$ & $0.032(2)$ \\
\hline$O(21 W)$ & $1.4430(5)$ & $1.5452(5)$ & $-0.6362(5)$ & $0.025(2)$ \\
\hline $\mathrm{O}(22 \mathrm{~W})$ & $1.4591(6)$ & $1.7193(6)$ & $-0.6643(6)$ & $0.034(2)$ \\
\hline$O(23 W)$ & $1.2695(5)$ & $1.9684(5)$ & $-0.5448(5)$ & $0.023(2)$ \\
\hline$O(24 W)$ & $0.9946(5)$ & $1.0763(5)$ & $0.0839(4)$ & $0.024(2)$ \\
\hline$O(25 W)$ & $1.0822(5)$ & $1.8712(6)$ & $-0.1659(5)$ & $0.029(2)$ \\
\hline$O(26 W)$ & $0.9293(5)$ & $2.0671(7)$ & $-0.3227(7)$ & $0.044(3)$ \\
\hline$O(27 W)$ & $0.3625(8)$ & $2.0292(10)$ & $-0.4640(7)$ & $0.054(4)$ \\
\hline $\mathrm{O}(28 \mathrm{~W})$ & $0.8583(5)$ & $1.2181(5)$ & $-0.0564(5)$ & $0.032(2)$ \\
\hline$O(29 W)$ & $1.3804(5)$ & $1.7421(5)$ & $-0.5120(5)$ & $0.027(2)$ \\
\hline$O(30 W)$ & $1.2955(7)$ & $1.8226(6)$ & $-0.4065(5)$ & $0.036(2)$ \\
\hline$O(31 W)$ & $1.5926(6)$ & $1.5488(5)$ & $-0.6368(5)$ & $0.035(2)$ \\
\hline$O(32 W)$ & $0.8887(6)$ & $1.6447(7)$ & $-0.4785(4)$ & $0.037(2)$ \\
\hline
\end{tabular}




\begin{tabular}{|c|c|c|c|c|}
\hline$O(33 W)$ & $1.1597(8)$ & $1.4576(8)$ & $-0.4611(7)$ & $0.064(4)$ \\
\hline$O(34 W)$ & $0.7954(5)$ & $1.4784(7)$ & $0.0783(5)$ & $0.039(3)$ \\
\hline$O(35 W)$ & $0.8208(7)$ & $1.0959(8)$ & $0.0648(7)$ & $0.045(3)$ \\
\hline$O(36 W)$ & $0.9053(5)$ & $1.9086(5)$ & $-0.2065(5)$ & $0.031(2)$ \\
\hline$O(37 W)$ & $0.4186(6)$ & $1.8396(9)$ & $-0.4516(7)$ & $0.055(4)$ \\
\hline $\mathrm{O}(38 \mathrm{~W})$ & $1.3962(5)$ & $1.6029(9)$ & $-0.3540(6)$ & $0.059(4)$ \\
\hline$O(39 w)$ & $1.1847(6)$ & $1.4047(6)$ & $-0.3161(8)$ & $0.058(4)$ \\
\hline$O(40 W)$ & $1.5258(6)$ & $0.9876(7)$ & $-0.0971(7)$ & $0.051(3)$ \\
\hline$O(41 W)$ & $0.8412(6)$ & $1.0726(7)$ & $-0.0598(6)$ & $0.048(3)$ \\
\hline $\mathrm{O}(42 W)$ & $0.5730(8)$ & $1.7348(9)$ & $-0.3287(8)$ & $0.072(5)$ \\
\hline$O(43 W)$ & $0.7666(7)$ & $1.6516(6)$ & $-0.0742(7)$ & $0.058(4)$ \\
\hline $\mathrm{O}(44 \mathrm{~W})$ & $0.8065(7)$ & $1.8546(6)$ & $-0.1797(7)$ & $0.049(3)$ \\
\hline$O(45 W)$ & $1.2589(8)$ & $0.2267(10)$ & $0.0455(8)$ & $0.068(4)$ \\
\hline$O(46 W)$ & $1.0361(5)$ & $1.6009(5)$ & $0.0698(4)$ & $0.024(2)$ \\
\hline$O(47 W)$ & $1.5826(6)$ & $1.0426(6)$ & $-0.0058(5)$ & $0.039(2)$ \\
\hline $\mathrm{O}(48 \mathrm{~W})$ & $1.6481(5)$ & $1.0570(5)$ & $-0.3243(5)$ & $0.025(2)$ \\
\hline$O(49 W)$ & $0.8451(5)$ & $1.0380(6)$ & $-0.1850(5)$ & $0.039(2)$ \\
\hline $\mathrm{O}(50 \mathrm{~W})$ & $0.8506(8)$ & $1.1940(8)$ & $-0.1954(5)$ & $0.049(3)$ \\
\hline$O(51 W)$ & $0.7700(8)$ & $1.3705(7)$ & $0.0752(6)$ & $0.045(3)$ \\
\hline$O(52 W)$ & $0.7766(8)$ & $0.2208(7)$ & $0.0850(7)$ & $0.058(4)$ \\
\hline$O(53 W)$ & $1.5142(6)$ & $1.1855(8)$ & $-0.2127(9)$ & $0.058(4)$ \\
\hline$O(54 W)$ & $0.3331(7)$ & $0.7868(13)$ & $0.2447(7)$ & $0.072(5)$ \\
\hline$O(55 W)$ & $1.6983(10)$ & $0.9131(9)$ & $-0.0422(7)$ & $0.073(5)$ \\
\hline$O(56 W)$ & $0.7764(9)$ & $1.7987(9)$ & $-0.5523(8)$ & $0.047(4)$ \\
\hline$O(57 W)$ & $0.5215(9)$ & $1.1194(10)$ & $0.1409(10)$ & $0.089(6)$ \\
\hline $\mathrm{O}(58 \mathrm{~W})$ & $0.3676(6)$ & $1.1694(8)$ & $0.2332(6)$ & $0.057(4)$ \\
\hline $\mathrm{O}(59 W)$ & $0.9814(10)$ & $1.3779(10)$ & $-0.4098(9)$ & $0.021(4)$ \\
\hline $\mathrm{O}(59 \mathrm{~V})$ & $0.9895(9)$ & $1.3388(11)$ & $-0.4094(9)$ & $0.025(4)$ \\
\hline $\mathrm{O}(60 \mathrm{~W})$ & $0.6639(10)$ & $1.6089(11)$ & $0.0182(10)$ & $0.090(7)$ \\
\hline$O(61 W)$ & $1.0785(10)$ & $1.2272(10)$ & $-0.4253(11)$ & $0.082(5)$ \\
\hline $\mathrm{O}(62 \mathrm{~W})$ & $0.6002(11)$ & $1.5267(11)$ & $-0.2585(9)$ & $0.031(4)$ \\
\hline$O(63 W)$ & $0.1991(9)$ & $1.0526(10)$ & $0.0816(14)$ & $0.030(5)$ \\
\hline $\mathrm{O}(63 \mathrm{~V})$ & $0.1983(14)$ & $1.0641(14)$ & $0.1095(13)$ & $0.041(7)$ \\
\hline $\mathrm{O}(64 \mathrm{~W})$ & $1.0539(6)$ & $1.5880(5)$ & $-0.4343(6)$ & $0.054(4)$ \\
\hline $\mathrm{O}(65 \mathrm{~W})$ & $0.4667(13)$ & $0.7548(19)$ & $0.0943(15)$ & $0.137(11)$ \\
\hline$O(66 W)$ & $1.6054(19)$ & $1.0204(19)$ & $-0.2066(13)$ & $0.065(9)$ \\
\hline
\end{tabular}




\begin{tabular}{|c|c|c|c|c|}
\hline$O(67 W)$ & $0.8765(6)$ & $1.7302(6)$ & $-0.0479(7)$ & $0.045(3)$ \\
\hline $\mathrm{O}(68 \mathrm{~W})$ & $0.3920(11)$ & $1.1219(12)$ & $0.1456(10)$ & $0.101(8)$ \\
\hline$O(69 W)$ & $0.4007(10)$ & $1.8857(11)$ & $-0.3237(10)$ & $0.024(4)$ \\
\hline $\mathrm{O}(69 \mathrm{~V})$ & $0.4047(12)$ & $1.8513(14)$ & $-0.3167(11)$ & $0.034(5)$ \\
\hline$O(70 W)$ & $0.6202(11)$ & $1.5512(15)$ & $-0.0935(16)$ & $0.051(7)$ \\
\hline$O(71 W)$ & 1.0000 & 0.5000 & 0.5000 & $0.078(7)$ \\
\hline $\mathrm{O}(72 \mathrm{~W})$ & $1.6820(11)$ & $1.0916(12)$ & $-0.1164(12)$ & $0.046(6)$ \\
\hline$O(73 W)$ & $0.6778(9)$ & $1.6807(10)$ & $-0.2063(10)$ & $0.087(6)$ \\
\hline $\mathrm{O}(74 \mathrm{~W})$ & $0.6739(14)$ & $1.3379(16)$ & $-0.2305(15)$ & $0.058(7)$ \\
\hline$O(75 W)$ & $0.6490(15)$ & $1.1373(12)$ & $0.0503(11)$ & $0.055(6)$ \\
\hline $\mathrm{O}(76 \mathrm{~W})$ & $1.0287(6)$ & $1.4596(5)$ & $-0.3470(7)$ & $0.068(6)$ \\
\hline$O(77 W)$ & $0.9948(7)$ & $1.0664(7)$ & $-0.2180(6)$ & $0.051(3)$ \\
\hline $\mathrm{H}(\mathrm{OA})$ & $1.1146(10)$ & $0.8559(10)$ & $-0.4130(9)$ & $0.038(4)$ \\
\hline $\mathrm{H}(\mathrm{OB})$ & $1.0926(11)$ & $0.9347(13)$ & $-0.4235(11)$ & $0.046(4)$ \\
\hline$H(1 A)$ & $1.1410(10)$ & $0.7059(11)$ & $-0.2226(9)$ & $0.039(4)$ \\
\hline$H(1 B)$ & $1.1752(10)$ & $0.7009(11)$ & $-0.1625(10)$ & $0.040(4)$ \\
\hline$H(2 A)$ & $1.2469(10)$ & $0.6977(11)$ & $-0.3148(10)$ & $0.042(4)$ \\
\hline $\mathrm{H}(2 \mathrm{~B})$ & $1.3036(18)$ & $0.6791(19)$ & $-0.2626(16)$ & $0.030(6)$ \\
\hline $\mathrm{H}(3 \mathrm{~A})$ & $1.2748(15)$ & $0.8451(17)$ & $-0.2189(14)$ & $0.069(6)$ \\
\hline$H(3 B)$ & $1.316(3)$ & $0.759(3)$ & $-0.196(2)$ & $0.049(9)$ \\
\hline$H(4 A)$ & $1.1746(9)$ & $1.3051(9)$ & $-0.1489(8)$ & $0.031(3)$ \\
\hline $\mathrm{H}(4 \mathrm{~B})$ & $1.1179(10)$ & $1.2817(11)$ & $-0.1371(9)$ & $0.040(4)$ \\
\hline$H(5 A)$ & $1.3669(9)$ & $1.2864(10)$ & $-0.3677(8)$ & $0.033(3)$ \\
\hline$H(5 B)$ & $1.3480(9)$ & $1.3077(9)$ & $-0.3126(9)$ & $0.034(3)$ \\
\hline$H(6 A)$ & $0.8407(10)$ & $1.8385(10)$ & $-0.4594(9)$ & $0.038(4)$ \\
\hline$H(6 B)$ & $0.8467(11)$ & $1.7781(11)$ & $-0.3853(11)$ & $0.044(4)$ \\
\hline$H(7 A)$ & $0.9869(11)$ & $1.1761(11)$ & $-0.3883(10)$ & $0.044(4)$ \\
\hline$H(7 B)$ & $0.9102(16)$ & $1.2334(16)$ & $-0.3888(14)$ & $0.065(6)$ \\
\hline$H(8 A)$ & $1.6004(9)$ & $1.5390(10)$ & $-0.4772(8)$ & $0.033(3)$ \\
\hline $\mathrm{H}(8 \mathrm{~B})$ & $1.5673(10)$ & $1.6279(11)$ & $-0.5099(9)$ & $0.039(4)$ \\
\hline$H(9 A)$ & $1.0400(10)$ & $1.6598(11)$ & $-0.0693(9)$ & $0.039(4)$ \\
\hline$H(9 B)$ & $1.0250(10)$ & $1.6308(10)$ & $-0.1147(6)$ & $0.042(4)$ \\
\hline$H(10 A)$ & $0.9987(9)$ & $1.7658(9)$ & $-0.0551(8)$ & $0.032(3)$ \\
\hline $\mathrm{H}(10 \mathrm{~B})$ & $1.0803(9)$ & $1.7165(9)$ & $-0.0395(8)$ & $0.032(3)$ \\
\hline$H(11 A)$ & $1.0991(8)$ & $1.9798(9)$ & $-0.5388(8)$ & $0.037(4)$ \\
\hline$H(11 B)$ & $1.1095(9)$ & $1.9077(10)$ & $-0.5418(8)$ & $0.035(3)$ \\
\hline
\end{tabular}




\begin{tabular}{|c|c|c|c|c|}
\hline$H(12 A)$ & $1.0853(10)$ & $1.3195(10)$ & $-0.3805(9)$ & $0.037(4)$ \\
\hline $\mathrm{H}(12 \mathrm{~B})$ & 1.1593(10) & $1.2422(11)$ & $-0.3379(9)$ & $0.037(4)$ \\
\hline$H(13 A)$ & $1.2440(9)$ & $1.8769(9)$ & $-0.6577(8)$ & $0.032(3)$ \\
\hline$H(13 B)$ & $1.2765(7)$ & $1.9244(9)$ & $-0.6575(8)$ & $0.033(3)$ \\
\hline$H(14 A)$ & $1.0112(10)$ & $1.8684(10)$ & $-0.3357(9)$ & $0.038(4)$ \\
\hline$H(14 B)$ & $1.0317(11)$ & $1.9198(11)$ & $-0.3243(10)$ & $0.042(4)$ \\
\hline$H(15 A)$ & $0.9206(11)$ & $1.4960(12)$ & $0.1110(10)$ & $0.045(4)$ \\
\hline$H(15 B)$ & $0.8458(7)$ & $1.5746(11)$ & $0.0813(10)$ & $0.049(4)$ \\
\hline$H(16 A)$ & $0.5590(12)$ & $1.8935(12)$ & $-0.3086(11)$ & $0.048(4)$ \\
\hline$H(16 B)$ & $0.4904(9)$ & $1.9772(8)$ & $-0.3556(10)$ & $0.045(4)$ \\
\hline$H(17 A)$ & $1.5494(15)$ & $1.6561(15)$ & $-0.4238(14)$ & $0.065(6)$ \\
\hline$H(17 B)$ & $1.5549(16)$ & $1.5992(16)$ & $-0.3475(11)$ & $0.040(8)$ \\
\hline$H(18 A)$ & $0.3068(9)$ & $2.0899(10)$ & $-0.6567(9)$ & $0.036(4)$ \\
\hline $\mathrm{H}(18 \mathrm{~B})$ & $0.3174(11)$ & $2.0175(12)$ & $-0.5915(11)$ & $0.046(4)$ \\
\hline$H(19 A)$ & $0.8751(10)$ & $1.8826(10)$ & $-0.3524(9)$ & $0.038(4)$ \\
\hline$H(19 B)$ & $0.8202(15)$ & $1.8845(17)$ & $-0.3086(14)$ & $0.069(6)$ \\
\hline$H(20 A)$ & $0.2958(11)$ & $2.2223(11)$ & $-0.6597(10)$ & $0.042(4)$ \\
\hline $\mathrm{H}(20 \mathrm{~B})$ & $0.342(2)$ & $2.205(2)$ & $-0.595(2)$ & $0.048(10)$ \\
\hline$H(20 C)$ & $0.360(2)$ & $2.211(2)$ & $-0.618(2)$ & $0.042(8)$ \\
\hline$H(21 A)$ & $1.3987(14)$ & $1.5550(13)$ & $-0.6230(12)$ & $0.055(5)$ \\
\hline$H(21 B)$ & $1.4328(17)$ & $1.5698(18)$ & $-0.6859(17)$ & $0.029(6)$ \\
\hline$H(22 A)$ & $1.4581(10)$ & $1.7439(11)$ & $-0.6379(10)$ & $0.043(4)$ \\
\hline$H(22 B)$ & $1.4782(15)$ & $1.7407(16)$ & $-0.7008(15)$ & $0.065(6)$ \\
\hline$H(23 A)$ & $1.2286(9)$ & $2.0102(10)$ & $-0.5837(9)$ & $0.034(3)$ \\
\hline$H(23 B)$ & $1.2608(10)$ & $1.9769(10)$ & $-0.5076(10)$ & $0.038(4)$ \\
\hline$H(24 A)$ & $0.9689(10)$ & $1.0979(10)$ & $0.1118(9)$ & $0.039(4)$ \\
\hline$H(24 B)$ & $1.0097(10)$ & $1.1099(10)$ & $0.0519(9)$ & $0.047(4)$ \\
\hline$H(25 A)$ & $1.1298(8)$ & $1.8326(12)$ & $-0.1370(11)$ & $0.058(5)$ \\
\hline$H(25 B)$ & $1.0877(10)$ & $1.9047(10)$ & $-0.2086(10)$ & $0.038(4)$ \\
\hline$H(26 A)$ & $0.8939(12)$ & $2.1225(13)$ & $-0.3555(10)$ & $0.048(4)$ \\
\hline$H(26 B)$ & $0.9685(14)$ & $2.060(2)$ & $-0.3449(17)$ & $0.040(8)$ \\
\hline$H(27 A)$ & $0.3321(9)$ & $2.0083(10)$ & $-0.4471(9)$ & $0.043(4)$ \\
\hline$H(27 B)$ & $0.3766(11)$ & $2.0385(12)$ & $-0.4312(9)$ & $0.049(5)$ \\
\hline$H(28 A)$ & $0.8806(11)$ & $1.2293(13)$ & $-0.0939(8)$ & $0.053(5)$ \\
\hline$H(28 B)$ & $0.8535(11)$ & $1.2533(13)$ & $-0.0422(10)$ & $0.048(4)$ \\
\hline$H(29 A)$ & $1.4078(9)$ & $1.7597(9)$ & $-0.5405(9)$ & $0.033(3)$ \\
\hline
\end{tabular}




\begin{tabular}{|c|c|c|c|c|}
\hline$H(29 B)$ & $1.3530(13)$ & $1.7356(13)$ & $-0.5386(12)$ & $0.053(5)$ \\
\hline$H(30 A)$ & $1.2828(10)$ & $1.8689(11)$ & $-0.4052(9)$ & $0.040(4)$ \\
\hline $\mathrm{H}(30 \mathrm{~B})$ & $1.2802(14)$ & $1.7905(15)$ & $-0.3703(14)$ & $0.064(6)$ \\
\hline$H(31 A)$ & $1.5847(14)$ & $1.5813(13)$ & $-0.6851(7)$ & $0.063(6)$ \\
\hline$H(31 B)$ & $1.6179(14)$ & $1.4947(8)$ & $-0.6250(14)$ & $0.071(7)$ \\
\hline$H(32 A)$ & $0.8637(10)$ & $1.6252(11)$ & $-0.4473(9)$ & $0.047(4)$ \\
\hline$H(32 B)$ & $0.881(2)$ & $1.654(2)$ & $-0.5240(10)$ & $0.050(9)$ \\
\hline $\mathrm{H}(32 \mathrm{C})$ & $0.8542(16)$ & $1.6976(11)$ & $-0.5133(14)$ & $0.037(7)$ \\
\hline$H(33 A)$ & $1.1715(11)$ & $1.4677(11)$ & $-0.5043(7)$ & $0.046(4)$ \\
\hline $\mathrm{H}(33 \mathrm{~B})$ & $1.1268(19)$ & $1.5042(14)$ & $-0.4603(18)$ & $0.035(8)$ \\
\hline $\mathrm{H}(33 \mathrm{C})$ & $1.1113(14)$ & $1.490(3)$ & $-0.461(3)$ & $0.063(14)$ \\
\hline$H(34 A)$ & $0.8229(10)$ & $1.4541(11)$ & $0.1232(7)$ & $0.047(4)$ \\
\hline$H(34 B)$ & $0.7467(8)$ & $1.5150(15)$ & $0.0737(15)$ & $0.077(7)$ \\
\hline$H(35 A)$ & $0.8173(12)$ & $1.0547(14)$ & $0.1021(12)$ & $0.052(5)$ \\
\hline$H(35 B)$ & $0.8078(15)$ & $1.1365(16)$ & $0.0750(14)$ & $0.021(5)$ \\
\hline$H(36 A)$ & $0.8725(10)$ & $1.8901(13)$ & $-0.1961(11)$ & $0.055(5)$ \\
\hline$H(36 B)$ & $0.8881(11)$ & $1.9470(9)$ & $-0.1926(10)$ & $0.046(4)$ \\
\hline$H(37 A)$ & $0.4397(12)$ & $1.8456(13)$ & $-0.4903(9)$ & $0.015(5)$ \\
\hline$H(37 B)$ & $0.3708(10)$ & $1.8820(15)$ & $-0.4786(15)$ & $0.033(7)$ \\
\hline$H(38 A)$ & $1.3551(16)$ & $1.597(3)$ & $-0.351(2)$ & $0.043(9)$ \\
\hline$H(38 B)$ & $1.4179(18)$ & $1.5605(16)$ & $-0.3076(9)$ & $0.039(7)$ \\
\hline$H(38 C)$ & $1.3463(9)$ & $1.629(3)$ & $-0.374(2)$ & $0.051(11)$ \\
\hline$H(39 A)$ & $1.1651(15)$ & $1.4573(9)$ & $-0.3249(14)$ & $0.026(6)$ \\
\hline$H(39 B)$ & $1.2233(14)$ & $1.3739(18)$ & $-0.2763(12)$ & $0.037(7)$ \\
\hline$H(40 A)$ & $1.4819(9)$ & $1.0174(12)$ & $-0.1309(9)$ & $0.053(5)$ \\
\hline$H(40 B)$ & $1.555(3)$ & $0.9370(18)$ & $-0.094(3)$ & $0.078(15)$ \\
\hline$H(41 A)$ & $0.826(2)$ & $1.0385(19)$ & $-0.0271(16)$ & $0.043(8)$ \\
\hline $\mathrm{H}(41 \mathrm{~B})$ & $0.813(3)$ & $1.085(4)$ & $-0.100(2)$ & $0.042(16)$ \\
\hline $\mathrm{H}(41 \mathrm{C})$ & $0.7941(19)$ & $1.119(3)$ & $-0.078(4)$ & $0.042(16)$ \\
\hline $\mathrm{H}(42 \mathrm{~A})$ & $0.5974(15)$ & $1.6981(14)$ & $-0.3442(15)$ & $0.030(6)$ \\
\hline $\mathrm{H}(42 \mathrm{~B})$ & $0.604(2)$ & $1.709(2)$ & $-0.2848(14)$ & $0.057(10)$ \\
\hline$H(43 A)$ & $0.790(2)$ & $1.669(2)$ & $-0.1091(16)$ & $0.047(9)$ \\
\hline $\mathrm{H}(43 \mathrm{~B})$ & $0.752(2)$ & $1.6944(17)$ & $-0.066(2)$ & $0.049(9)$ \\
\hline $\mathrm{H}(44 \mathrm{~A})$ & $0.7892(12)$ & $1.8406(12)$ & $-0.2067(11)$ & $0.051(5)$ \\
\hline $\mathrm{H}(44 \mathrm{~B})$ & $0.768(2)$ & $1.900(2)$ & $-0.180(3)$ & $0.079(15)$ \\
\hline$H(45 A)$ & $1.2616(18)$ & $0.2633(16)$ & $0.0028(11)$ & $0.035(7)$ \\
\hline
\end{tabular}




\begin{tabular}{|c|c|c|c|c|}
\hline$H(45 B)$ & $1.2092(10)$ & $0.245(2)$ & $0.045(2)$ & $0.047(9)$ \\
\hline$H(46 A)$ & $1.0207(9)$ & $1.6409(10)$ & $0.0816(8)$ & $0.033(3)$ \\
\hline$H(46 B)$ & $1.0539(9)$ & $1.5513(9)$ & $0.1117(8)$ & $0.030(3)$ \\
\hline$H(47 A)$ & $1.6227(10)$ & $1.0011(11)$ & $0.0327(9)$ & $0.058(5)$ \\
\hline$H(47 B)$ & $1.551(3)$ & $1.0932(18)$ & $-0.010(3)$ & $0.076(14)$ \\
\hline$H(47 C)$ & $1.604(3)$ & $1.049(3)$ & $-0.0441(17)$ & $0.064(12)$ \\
\hline$H(48 A)$ & $1.6088(10)$ & $1.1100(10)$ & $-0.3520(9)$ & $0.035(4)$ \\
\hline $\mathrm{H}(48 \mathrm{~B})$ & $1.6462(10)$ & $1.0267(11)$ & $-0.3422(9)$ & $0.038(4)$ \\
\hline$H(49 A)$ & $0.8169(11)$ & $1.0869(9)$ & $-0.2267(8)$ & $0.054(5)$ \\
\hline$H(49 B)$ & $0.8163(10)$ & $1.0202(12)$ & $-0.1580(10)$ & $0.052(5)$ \\
\hline$H(50 A)$ & $0.8305(11)$ & $1.2237(11)$ & $-0.2438(6)$ & $0.048(4)$ \\
\hline$H(50 B)$ & $0.8847(13)$ & $1.2064(13)$ & $-0.1879(11)$ & $0.052(5)$ \\
\hline$H(51 A)$ & $0.7921(14)$ & $1.3539(15)$ & $0.0431(13)$ & $0.019(5)$ \\
\hline$H(51 B)$ & $0.7805(14)$ & $1.4101(12)$ & $0.0688(14)$ & $0.023(5)$ \\
\hline$H(52 A)$ & $0.7489(16)$ & $0.2672(13)$ & $0.0413(10)$ & $0.033(7)$ \\
\hline$H(52 B)$ & $0.7518(18)$ & $0.2229(19)$ & $0.1201(17)$ & $0.033(6)$ \\
\hline$H(53 A)$ & $1.4829(12)$ & $1.1701(15)$ & $-0.2211(14)$ & $0.023(5)$ \\
\hline$H(53 B)$ & $1.4856(15)$ & $1.2290(13)$ & $-0.2055(15)$ & $0.029(6)$ \\
\hline$H(54 A)$ & $0.2950(12)$ & $0.7992(13)$ & $0.2692(11)$ & $0.013(4)$ \\
\hline$H(54 B)$ & $0.3777(14)$ & $0.7625(14)$ & $0.2783(13)$ & $0.019(5)$ \\
\hline$H(55 A)$ & $1.7129(18)$ & $0.8940(19)$ & $0.0053(9)$ & $0.036(7)$ \\
\hline$H(55 B)$ & $1.7046(18)$ & $0.8691(14)$ & $-0.0444(16)$ & $0.032(6)$ \\
\hline$H(56 A)$ & $0.7496(16)$ & $1.7988(19)$ & $-0.5848(14)$ & $0.035(7)$ \\
\hline$H(56 B)$ & $0.7821(17)$ & $1.8436(13)$ & $-0.5732(15)$ & $0.034(7)$ \\
\hline$H(57 A)$ & $0.5614(12)$ & $1.0736(12)$ & $0.1781(11)$ & $0.026(6)$ \\
\hline$H(57 B)$ & $0.4820(17)$ & $1.112(3)$ & $0.144(2)$ & $0.052(10)$ \\
\hline$H(58 A)$ & $0.3224(9)$ & $1.1770(16)$ & $0.2431(14)$ & $0.024(5)$ \\
\hline$H(58 B)$ & $0.381(2)$ & $1.152(3)$ & $0.2007(18)$ & $0.053(10)$ \\
\hline$H(59 A)$ & $0.9485(18)$ & $1.388(2)$ & $-0.3816(17)$ & $0.041(9)$ \\
\hline$H(59 B)$ & $0.9818(19)$ & $1.4242(15)$ & $-0.4430(15)$ & $0.036(7)$ \\
\hline $\mathrm{H}(59 \mathrm{C})$ & $0.9589(19)$ & $1.365(2)$ & $-0.3870(18)$ & $0.033(9)$ \\
\hline$H(59 D)$ & $0.9799(17)$ & $1.2995(15)$ & $-0.4054(16)$ & $0.030(6)$ \\
\hline$H(60 A)$ & $0.6187(16)$ & $1.614(3)$ & $0.026(3)$ & $0.060(12)$ \\
\hline$H(60 B)$ & $0.680(2)$ & $1.636(2)$ & $0.030(2)$ & $0.050(9)$ \\
\hline$H(61 A)$ & $1.081(3)$ & $1.1818(19)$ & $-0.422(2)$ & $0.060(11)$ \\
\hline $\mathrm{H}(61 \mathrm{~B})$ & $1.1243(17)$ & $1.205(3)$ & $-0.398(3)$ & $0.071(14)$ \\
\hline
\end{tabular}




\begin{tabular}{|c|c|c|c|c|}
\hline$H(62 A)$ & $0.575(3)$ & $1.541(4)$ & $-0.227(2)$ & $0.080(15)$ \\
\hline$H(62 B)$ & $0.587(4)$ & $1.492(4)$ & $-0.224(3)$ & $0.095(19)$ \\
\hline$H(63 A)$ & $0.1553(12)$ & $1.1026(12)$ & $0.0673(14)$ & $0.024(6)$ \\
\hline$H(63 B)$ & $0.2347(19)$ & $1.065(3)$ & $0.086(2)$ & $0.055(10)$ \\
\hline$H(63 C)$ & $0.214(3)$ & $1.042(3)$ & $0.041(2)$ & $0.057(10)$ \\
\hline$H(64 A)$ & $1.044(2)$ & $1.5481(17)$ & $-0.4022(16)$ & $0.042(8)$ \\
\hline$H(64 B)$ & $1.0840(16)$ & $1.6098(19)$ & $-0.4392(19)$ & $0.040(8)$ \\
\hline$H(64 C)$ & $1.045(2)$ & $1.610(2)$ & $-0.4836(8)$ & $0.053(10)$ \\
\hline$H(64 D)$ & $1.0778(16)$ & $1.5813(18)$ & $-0.3947(12)$ & $0.031(6)$ \\
\hline$H(65 A)$ & $0.500(2)$ & $0.723(3)$ & $0.1378(18)$ & $0.066(12)$ \\
\hline$H(65 B)$ & $0.422(2)$ & $0.781(4)$ & $0.105(3)$ & $0.035(14)$ \\
\hline$H(66 A)$ & $1.637(2)$ & $1.015(3)$ & $-0.2371(19)$ & $0.053(10)$ \\
\hline$H(66 B)$ & $1.554(3)$ & $1.048(3)$ & $-0.228(3)$ & $0.020(10)$ \\
\hline$H(67 A)$ & $0.8966(10)$ & $1.7509(11)$ & $-0.0306(9)$ & $0.040(4)$ \\
\hline$H(67 B)$ & $0.864(3)$ & $1.766(3)$ & $-0.0971(9)$ & $0.066(12)$ \\
\hline$H(68 A)$ & $0.3484(16)$ & $1.159(2)$ & $0.1107(18)$ & $0.046(9)$ \\
\hline$H(68 B)$ & $0.393(5)$ & $1.073(3)$ & $0.172(4)$ & $0.06(2)$ \\
\hline$H(69 A)$ & $0.3566(14)$ & $1.9005(14)$ & $-0.3469(12)$ & $0.060(5)$ \\
\hline$H(69 B)$ & $0.392(2)$ & $1.9243(17)$ & $-0.3116(18)$ & $0.040(8)$ \\
\hline$H(69 C)$ & $0.410(3)$ & $1.863(4)$ & $-0.279(4)$ & $0.081(15)$ \\
\hline$H(70 A)$ & $0.657(2)$ & $1.517(2)$ & $-0.107(2)$ & $0.017(9)$ \\
\hline $\mathrm{H}(70 \mathrm{~B})$ & $0.575(4)$ & $1.573(8)$ & $-0.122(6)$ & $0.09(4)$ \\
\hline$H(71 A)$ & $1.0414(14)$ & $0.503(2)$ & $0.502(2)$ & $0.053(10)$ \\
\hline$H(71 B)$ & $1.037(3)$ & $0.454(3)$ & $0.498(4)$ & $0.040(15)$ \\
\hline $\mathrm{H}(72 \mathrm{~A})$ & $1.680(2)$ & $1.127(2)$ & $-0.162(2)$ & $0.044(8)$ \\
\hline$H(72 B)$ & $1.730(2)$ & $1.048(4)$ & $-0.093(4)$ & $0.06(2)$ \\
\hline$H(73 A)$ & $0.7212(16)$ & $1.674(3)$ & $-0.221(2)$ & $0.061(11)$ \\
\hline$H(73 B)$ & $0.646(5)$ & $1.735(2)$ & $-0.220(6)$ & $0.07(3)$ \\
\hline$H(74 A)$ & $0.641(2)$ & $1.347(3)$ & $-0.202(2)$ & $0.020(10)$ \\
\hline$H(74 B)$ & $0.704(3)$ & $1.351(4)$ & $-0.258(3)$ & $0.046(17)$ \\
\hline $\mathrm{H}(74 \mathrm{C})$ & $0.666(5)$ & $1.305(4)$ & $-0.241(4)$ & $0.11(2)$ \\
\hline$H(75 A)$ & $0.6733(15)$ & $1.0890(13)$ & $0.0934(11)$ & $0.027(6)$ \\
\hline$H(75 B)$ & $0.633(4)$ & $1.183(3)$ & $0.054(4)$ & $0.044(16)$ \\
\hline$H(76 A)$ & $0.9825(11)$ & $1.4697(11)$ & $-0.3343(10)$ & $0.045(4)$ \\
\hline$H(76 B)$ & $1.021(2)$ & $1.458(2)$ & $-0.3876(13)$ & $0.038(8)$ \\
\hline$H(77 A)$ & $1.0392(13)$ & $1.0165(14)$ & $-0.1970(18)$ & $0.042(8)$ \\
\hline
\end{tabular}


$H(77 B)$

$0.9725(16)$

1.0674(19)

$-0.2542(13)$

$0.033(7)$ 
Table S6: Anisotropic displacement parameters $\left(\AA^{2}\right)$ for $\mathrm{Na} / \mathrm{K}\left\{\mathrm{U}_{24} \mathrm{Pp} \mathrm{p}_{12}\right\}$.

The anisotropic displacement factor exponent takes the form:

$-2 \pi^{2}\left[h^{2} a * 2 U_{11}+\ldots+2 h k a * b * U_{12}\right]$

$\begin{array}{llllll}\mathrm{U}_{11} & \mathrm{U}_{22} & \mathrm{U}_{33} & \mathrm{U}_{23} & \mathrm{U}_{13} & \mathrm{U}_{12}\end{array}$

$\mathrm{U}(1) \quad 0.011(2) \quad 0.012(2) \quad 0.011(2) \quad-0.0071(18) \quad 0.0024(17)-0.0063(18)$

$\mathrm{U}(2) \quad 0.013(2) \quad 0.010(2) \quad 0.010(2) \quad-0.0048(17) \quad 0.0017(17)-0.0056(18)$

$\mathrm{U}(3) \quad 0.010(2) \quad 0.0081(19) \quad 0.012(2) \quad-0.0052(17) \quad 0.0022(17)-0.0041(17)$

$\mathrm{U}(4) \quad 0.011(2) \quad 0.011(2) \quad 0.013(2) \quad-0.0073(18) \quad 0.0029(17)-0.0059(18)$

$\mathrm{U}(5) \quad 0.008(2) \quad 0.009(2) \quad 0.014(2) \quad-0.0074(17) \quad 0.0052(17)-0.0041(17)$

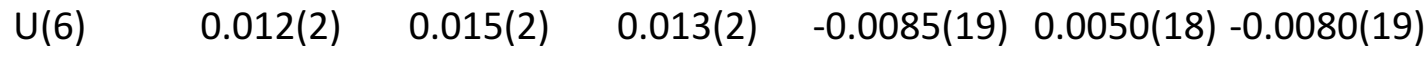

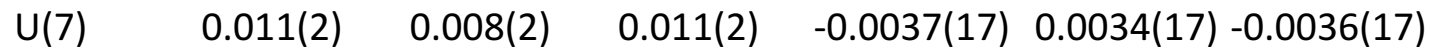

$\mathrm{U}(8) \quad 0.011(2) \quad 0.012(2) \quad 0.010(2) \quad-0.0043(18) \quad 0.0013(17)-0.0061(18)$

$\mathrm{U}(9) \quad 0.011(2) \quad 0.009(2) \quad 0.013(2) \quad-0.0064(18) \quad 0.0050(17)-0.0048(17)$

$\mathrm{U}(10) \quad 0.012(2) \quad 0.008(2) \quad 0.018(2) \quad-0.0076(18) \quad 0.0062(18)-0.0054(18)$

$\mathrm{U}(11) \quad 0.009(2) \quad 0.012(2) \quad 0.013(2) \quad-0.0068(18) \quad 0.0018(17)-0.0048(18)$

$\begin{array}{lllllll}\mathrm{U}(12) & 0.013(2) & 0.015(2) & 0.013(2) & -0.0091(19) & 0.0054(18) & -0.0088(19)\end{array}$

$\begin{array}{lllllll}\mathrm{I}(1) & 0.028(5) & 0.027(4) & 0.012(4) & -0.010(3) & 0.004(3) & -0.014(4)\end{array}$

$\begin{array}{lllllll}K(1) & 0.024(7) & 0.014(6) & 0.028(7) & -0.009(5) & 0.005(5) & -0.006(5)\end{array}$

$\begin{array}{lllllll}K(2) & 0.039(8) & 0.014(6) & 0.017(6) & -0.007(5) & 0.012(6) & -0.013(6)\end{array}$

$\begin{array}{lllllll}K(3) & 0.024(7) & 0.054(10) & 0.035(8) & -0.037(8) & 0.010(6) & -0.013(7)\end{array}$

$\begin{array}{lllllll}\mathrm{Na}(1) & 0.013(6) & 0.019(6) & 0.027(7) & -0.014(5) & 0.000(5) & -0.007(5)\end{array}$

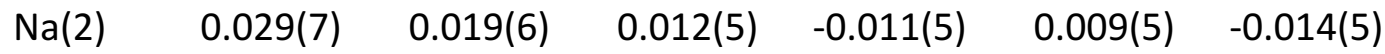

$\begin{array}{lllllll}\mathrm{Na}(3) & 0.020(6) & 0.017(6) & 0.023(7) & -0.013(5) & 0.006(5) & -0.004(5)\end{array}$

$\begin{array}{lllllll}\mathrm{Na}(4) & 0.012(5) & 0.010(5) & 0.019(6) & -0.009(4) & 0.002(4) & -0.003(4)\end{array}$

$\begin{array}{lllllll}\mathrm{Na}(5) & 0.020(6) & 0.019(6) & 0.025(7) & -0.007(5) & 0.006(5) & -0.014(5)\end{array}$

$\begin{array}{lllllll}\mathrm{Na}(6) & 0.022(6) & 0.022(6) & 0.014(6) & -0.011(5) & 0.009(5) & -0.011(5)\end{array}$

$\begin{array}{lllllll}\mathrm{Na}(7) & 0.024(7) & 0.015(5) & 0.016(6) & -0.006(5) & 0.004(5) & -0.007(5)\end{array}$

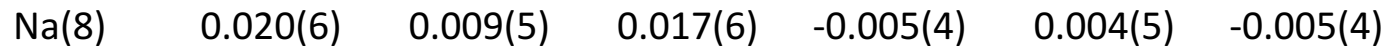

$\begin{array}{lllllll}\mathrm{Na}(9) & 0.019(6) & 0.020(6) & 0.022(6) & -0.012(5) & 0.002(5) & -0.009(5)\end{array}$

$\begin{array}{lllllll}\mathrm{Na}(10) & 0.021(7) & 0.029(7) & 0.023(7) & -0.017(6) & 0.007(5) & -0.011(6)\end{array}$

$\begin{array}{lllllll}\mathrm{Na}(11) & 0.024(7) & 0.033(7) & 0.045(9) & -0.031(7) & 0.016(6) & -0.023(6)\end{array}$

$\begin{array}{lllllll}\mathrm{Na}(12) & 0.028(7) & 0.020(6) & 0.021(7) & -0.011(5) & 0.009(5) & -0.013(5)\end{array}$

$\begin{array}{lllllll}\mathrm{Na}(13) & 0.034(8) & 0.028(7) & 0.016(6) & -0.006(5) & 0.002(5) & -0.022(6)\end{array}$

$\begin{array}{lllllll}\mathrm{Na}(14) & 0.050(10) & 0.033(8) & 0.031(8) & -0.024(7) & 0.023(7) & -0.025(7)\end{array}$ 


\begin{tabular}{|c|c|c|c|c|c|c|}
\hline ) & 7) & $0.029(7)$ & 7) & 5) & 5) & ( \\
\hline $\mathrm{Na}(16)$ & 061(18) & $.047(14)$ & $0.037(14)$ & $0.006(11)$ & $-0.012(12)$ & $-0.043(1$ \\
\hline (17) & $080(15)$ & $035(9)$ & 033(9) & $-0.011(8)$ & $.003(9)$ & $-0.028(10)$ \\
\hline$a(1$ & $58(13)$ & ) & 3) & 2) & 1) & - \\
\hline $\mathrm{Na}(19)$ & 060(13) & .051 & $0.055(12)$ & $-0.030(10)$ & $0.018(10)$ & $-0.024(10)$ \\
\hline 1) & $013(4)$ & $.014(3)$ & $0.015(4)$ & $-0.008(3)$ & $0.009(3)$ & $-0.010(3)$ \\
\hline 2) & 0 & & & -0 & & \\
\hline 3) & 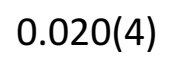 & ) & & $-c$ & & \\
\hline (4) & 011(4) & $0.013(4)$ & $0.018(4)$ & $-0.010(3)$ & $0.005(3)$ & $-0.009(3)$ \\
\hline 5) & & ) & & -0. & & \\
\hline 6) & 01 & 0.01 & & & 3) & 7(3) \\
\hline 7) & 012 & 01 & 4) & $-0 .($ & 0.00 & $8(3)$ \\
\hline 8) & 014 & .0 & & $-0 .($ & & \\
\hline (9) & 020(4) & $0.010(3)$ & 0.0 & $-0.004(3)$ & $0.007(3)$ & 7(3) \\
\hline 10 & 4) & 0.00 & 0.0 & -0.0 & -0.0 & (3) \\
\hline (11) & & & & & & \\
\hline 12 & 015 & $.012(3)$ & $0.009(4)$ & $-0.005(3)$ & $0.0 c$ & 5(3) \\
\hline (1) & ) & 0.0 & 4) & -0.0 & 3) & (3) \\
\hline (2) & & & & & & \\
\hline (3 & 01 & ) & & -0 . & 0.0 & (3) \\
\hline (4) & 016(4) & $0.018(3)$ & $0.017(4)$ & $-0.013(3)$ & $0.007(3)$ & $-0.007(3)$ \\
\hline$(5$ & 0 & 01 & & -0. & (3) & (3) \\
\hline$O(6)$ & 3) & 0.017 & 0.0 & -0.0 & (3) & $5(3)$ \\
\hline $\mathrm{O}(7)$ & 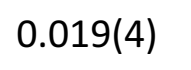 & 0.023 & 4) & -0.0 & (3) & $4(3)$ \\
\hline$(8$ & & & & -0.01 & & \\
\hline$O(9)$ & 014(3) & $0.015(3)$ & $0.012(3)$ & $-0.005(3)$ & $0.004(3)$ & $-0.009(3)$ \\
\hline $\mathrm{O}(1 \mathrm{C}$ & $.015(4)$ & $0.015(3)$ & $0.014(3)$ & $-0.005(3)$ & $0.004(3)$ & $-0.009(3)$ \\
\hline $\mathrm{O}(1$ & $.016(4)$ & $0.013(3)$ & & -0.00 & 0.00 & \\
\hline $\mathrm{O}(12)$ & $0.017(3)$ & $0.014(3)$ & $0.011(3)$ & $-0.007(3)$ & $0.004(3)$ & $-0.008(3)$ \\
\hline $\mathrm{O}(13)$ & $0.014(3)$ & $0.013(3)$ & $0.013(3)$ & $-0.006(3)$ & $0.004(3)$ & $-0.005(3)$ \\
\hline $\mathrm{O}(14)$ & $0.021(4)$ & $0.016(3)$ & $0.010(3)$ & $-0.008(3)$ & $0.005(3)$ & $-0.007(3)$ \\
\hline$O(15)$ & $0.022(4)$ & $0.017(3)$ & $0.011(3)$ & $-0.006(3)$ & $0.005(3)$ & $-0.008(3)$ \\
\hline $\mathrm{O}(16$ & $11(3)$ & 011(3) & 015(3) & $-0.007(3)$ & 0.00 & $-0.004(3)$ \\
\hline $\mathrm{O}(17)$ & $0.012(3)$ & $0.012(3)$ & $0.019(4)$ & $-0.010(3)$ & $0.004(3)$ & $-0.005(3)$ \\
\hline $\mathrm{O}(18)$ & $0.011(3)$ & $0.010(3)$ & $0.016(3)$ & $-0.007(3)$ & $0.005(3)$ & $-0.007(3)$ \\
\hline O(19) & $0.011(3)$ & $0.015(3)$ & $0.019(4)$ & $-0.012(3)$ & $0.003(3)$ & $-0.004(3)$ \\
\hline
\end{tabular}




\begin{tabular}{|c|c|c|c|c|c|c|}
\hline ) & 008(3) & 3) & 4) & $-0 .($ & & \\
\hline (21) & ) & ) & 4) & ח & 3) & - 0 \\
\hline (22) & $017(4)$ & $021(4)$ & 014(3) & $-0.011(3)$ & $.007(3)$ & $-0.010(3)$ \\
\hline (23) & $.026(4)$ & $.012(3)$ & $.012(3)$ & $-0.003(3)$ & $0.001(3)$ & $0.009(3)$ \\
\hline 24 & 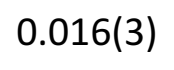 & & & & & \\
\hline (25) & 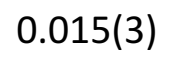 & ) & 3) & 3) & 3) & $0(3)$ \\
\hline$(26$ & $.016(4)$ & .01 & 4) & -0.0 & 3) & 7(3) \\
\hline (27) & 01 & 0.00 & 0.02 & & & \\
\hline (28) & $.014(3)$ & $0.014(3)$ & $0.016(4)$ & $-0.008(3)$ & $0.001(3)$ & $-0.005(3)$ \\
\hline$(29$ & 01 & $0 . c$ & 0.0 & -0 & 3) & (3) \\
\hline$(30$ & & & & & & \\
\hline $\mathrm{O}(31)$ & $0.021(4)$ & $0.021(4)$ & $0.016(4)$ & $-0.014(3)$ & $0.006(3)$ & $-0.011(3)$ \\
\hline (32) & $.014(4)$ & $0.024(4)$ & 0.0 & -0 & 3) & (3) \\
\hline $\mathrm{O}(33$ & 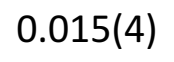 & ) & & & & \\
\hline (34) & .018 & .01 & .01 & -0.0 & $-0.0 c$ & $-0.004(3)$ \\
\hline (35) & .01 & 0.0 & 0.0 & $-0 .($ & $0.003(3)$ & $-0.004(3)$ \\
\hline$O(36)$ & $.016(4)$ & $0.015(3)$ & $0.016(4)$ & $-0.008(3)$ & $0.006(3)$ & $8(3)$ \\
\hline $\mathrm{O}(37)$ & $.016(4)$ & $0.012(3)$ & $0.016(4)$ & $-0.004(3)$ & $0.000(3)$ & $-0.007(3)$ \\
\hline $\mathrm{O}(38$ & & & & & & \\
\hline $\mathrm{O}(39$ & 017 & .005 & 3) & -0.0 & (3) & 2(3) \\
\hline $\mathrm{O}(40)$ & $0.014(3)$ & $0.014(3)$ & $0.021(4)$ & $-0.008(3)$ & $0.001(3)$ & $-0.008(3)$ \\
\hline (41 & 015 & & (4) & (3) & $.005(3)$ & 9(3) \\
\hline$(42$ & 01 & ) & & & & $6(3)$ \\
\hline $\mathrm{O}(43)$ & $.016(4)$ & $0.016(3)$ & $0.014(4)$ & $-0.007(3)$ & $0.005(3)$ & $-0.007(3)$ \\
\hline (44) & $014(3)$ & $.014(3)$ & $0.017(4)$ & $-0.007(3)$ & $-0.001(3)$ & $-0.007(3)$ \\
\hline (45) & 013(3) & .018 & 0.02 & -0.01 & & $8(3)$ \\
\hline $\mathrm{O}(46)$ & $0.012(3)$ & $0.019(3)$ & $0.018(4)$ & $-0.012(3)$ & $.009(3)$ & $-0.009(3)$ \\
\hline 4 & & ) & & 3) & & \\
\hline $\mathrm{O}(48)$ & $0.013(3)$ & $0.013(3)$ & $0.025(4)$ & $-0.011(3)$ & $0.007(3)$ & $-0.006(3)$ \\
\hline $\mathrm{O}(49)$ & $0.018(4)$ & $0.021(4)$ & $0.019(4)$ & $-0.016(3)$ & $0.010(3)$ & $-0.011(3)$ \\
\hline O(50) & & $.013(4)$ & $0.042(5)$ & $-0.006(4)$ & $.007(4)$ & $-0.004(3)$ \\
\hline $\mathrm{O}(51)$ & $0.013(4)$ & $0.024(4)$ & $0.020(4)$ & $-0.015(3)$ & $.003(3)$ & $-0.004(3)$ \\
\hline $\mathrm{O}(52)$ & $018(4)$ & $0.011(3)$ & $0.011(3)$ & $-0.004(3)$ & $0.003(3)$ & $-0.008(3)$ \\
\hline $\mathrm{O}(53)$ & $.009(3)$ & $0.019(3)$ & $0.013(3)$ & $-0.004(3)$ & $0.002(3)$ & $-0.006(3)$ \\
\hline O(54) & $0.017(4)$ & $0.013(3)$ & $0.018(4)$ & $-0.008(3)$ & $0.009(3)$ & $-0.010(3)$ \\
\hline O(55) & $0.014(3)$ & $0.013(3)$ & $0.015(4)$ & $-0.006(3)$ & $0.002(3)$ & $-0.005(3)$ \\
\hline
\end{tabular}




\begin{tabular}{|c|c|c|c|c|c|c|}
\hline (56) & ) & 3) & & & & \\
\hline (57) & 014(4) & 01 & $.020(4)$ & $.006(3)$ & 006(3) & 3) \\
\hline (58) & 01 & 013(3) & $020(4)$ & (3) & $006(3)$ & $-0.004(3)$ \\
\hline (59) & $.017(4)$ & $.013(3)$ & $.014(3)$ & $0.008(3)$ & $.002(3)$ & $.0 .005(3)$ \\
\hline$O(60)$ & & & & & & \\
\hline (61 & ) & 3) & (4) & 3) & & $5(3)$ \\
\hline (62) & $026(4)$ & $.018(3)$ & $.021(4)$ & -0 . & 3) & \\
\hline (63 & . & 4) & l(4) & & & \\
\hline (64) & $.022(4)$ & $.018(3)$ & $0.017(4)$ & $1(3)$ & $0.006(3)$ & -0.0 \\
\hline (65 & 04 & 0.0 & 0.1 & 3) & ) & \\
\hline$(66$ & & 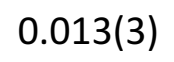 & & & & \\
\hline O(67) & $0.020(4)$ & $0.022(4)$ & $0.015(4)$ & $-0.013(3)$ & $0.010(3)$ & $-0.013(3)$ \\
\hline 68 & 01 & (4) & $3(4)$ & 3) & & (3) \\
\hline T & 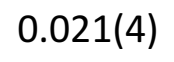 & ) & & & & $O(3)$ \\
\hline (70) & .01 & $.017(4)$ & $.022(4)$ & -0.0 & 0.00 & $-0.004(3)$ \\
\hline (71) & 01 & $0.013(3)$ & $0.012(3)$ & (3) & $0.004(3)$ & $-0.005(3)$ \\
\hline$(72)$ & $.008(3)$ & $0.019(3)$ & $0.017(3)$ & 1(3) & $0.005(3)$ & $-0.008(3)$ \\
\hline$O(73)$ & 014(3) & $0.013(3)$ & $0.017(3)$ & $-0.007(3)$ & $0.007(3)$ & -0.0 \\
\hline $\mathrm{O}(74$ & & & & & & \\
\hline (75 & 016 & $.014(3)$ & $023(4)$ & 2(3) & $0.002(3)$ & $-0 . c$ \\
\hline $\mathrm{O}(76)$ & $0.014(3)$ & $0.014(3)$ & $0.011(3)$ & $-0.006(3)$ & $0.004(3)$ & $-0.005(3)$ \\
\hline (77) & 011 & $.013(3)$ & $.016(4)$ & $3(3)$ & $0.005(3)$ & $-0.005(3)$ \\
\hline (78) & 01 & .01 & (3) & 3) & & -0.0 \\
\hline O(79) & $.021(4)$ & $0.011(3)$ & $0.014(3)$ & $-0.007(3)$ & $0.004(3)$ & $-0.007(3)$ \\
\hline $\mathrm{O}(80)$ & $.019(4)$ & $.015(3)$ & $0.017(4)$ & $-0.008(3)$ & $0.007(3)$ & $-0.011(3)$ \\
\hline $\mathrm{O}(81)$ & $.024(4)$ & $0.012(3)$ & $0.013(4)$ & & & \\
\hline $\mathrm{O}(82)$ & $0.015(3)$ & $0.013(3)$ & $0.017(4)$ & $-0.011(3)$ & $0.003(3)$ & $-0.002(3)$ \\
\hline 83 & 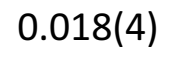 & 3) & & (3) & & -0.0 \\
\hline O(84) & $0.020(4)$ & $0.017(3)$ & $0.017(4)$ & $-0.008(3)$ & $0.001(3)$ & $-0.006(3)$ \\
\hline $\mathrm{O}(85)$ & $.015(3)$ & $0.012(3)$ & $0.015(4)$ & $-0.004(3)$ & $0.001(3)$ & $-0.006(3)$ \\
\hline O(86) & $33(5)$ & $.015(4)$ & $022(4)$ & $-0.008(3)$ & $0.006(4)$ & $-0.004(3)$ \\
\hline $\mathrm{O}(87)$ & $0.018(4)$ & $0.016(3)$ & $0.014(4)$ & $-0.006(3)$ & $-0.004(3)$ & $-0.008(3)$ \\
\hline O(88) & 063 & $0.062(7)$ & $0.022(5)$ & $-0.030(5)$ & $0.022(5)$ & $-0.045(6)$ \\
\hline O(89) & $.031(5)$ & $0.027(4)$ & $0.022(4)$ & $-0.014(3)$ & $0.016(3)$ & $-0.025(4)$ \\
\hline O(90) & $0.022(5)$ & $0.080(8)$ & $0.041(6)$ & $-0.038(6)$ & $0.015(4)$ & $-0.033(5)$ \\
\hline $\mathrm{O}(1 \mathrm{l})$ & $0.036(5)$ & $0.028(4)$ & $0.020(4)$ & $-0.009(3)$ & $0.001(4)$ & $-0.019(4)$ \\
\hline
\end{tabular}




\begin{tabular}{|c|c|c|c|c|c|c|}
\hline 21) & ) & ) & & & & \\
\hline & 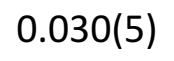 & & & & & \\
\hline & & & & & & \\
\hline 11 & & & & & & \\
\hline $2 W)$ & 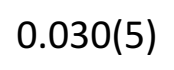 & & & & & \\
\hline (f) & & & & & & \\
\hline $4 \mathrm{n}$ & 01 & & & & 3) & \\
\hline 51 & & & & & & \\
\hline $6 \mathrm{~W}$ & & & & & & \\
\hline (7W) & $035(6)$ & $023(4)$ & $0.023(5)$ & 4) & (4) & $.006(4)$ \\
\hline $8 \mathrm{n}$ & & & & & & \\
\hline$(9 n$ & & & & & & \\
\hline 10 & 02 & t) & & & & \\
\hline 11 & 1 & & & & & \\
\hline 12 & 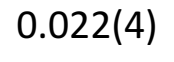 & & & & & \\
\hline 13 & 02 & & & & & \\
\hline 14 & & & & & & \\
\hline 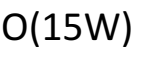 & ) & 5) & & & & \\
\hline$(16$ & 01 & & & & & \\
\hline (17) & & & & & & \\
\hline $18 \quad$ & & & & & & \\
\hline (19) & 023(4) & 4) & $0.029(5)$ & $-0 . c$ & (4) & -0.0 \\
\hline 20 & ) & & & & & \\
\hline (21 & & & & & & \\
\hline$(22)$ & 03 & 5) & & & & \\
\hline 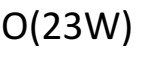 & . & & & 1 & & \\
\hline 24 & & & 4) & & & \\
\hline$O(25 \mathrm{~V}$ & ) & ) & & & & (5) \\
\hline 20 & & & & & & \\
\hline$O(27 \mathrm{~W}$ & $.061(8)$ & (110(11) & $0.063(8)$ & $-0.066(8)$ & $0.042(7)$ & (9) \\
\hline $\mathrm{O}(28 \mathrm{~V}$ & $.032(5)$ & $.021(4)$ & $0.031(5)$ & $-0.010(4)$ & (4) & $-0.008(4)$ \\
\hline te & & & & & & \\
\hline $\mathrm{O}(30 \mathrm{~W})$ & & & & & & \\
\hline 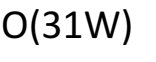 & $0.033(5)$ & & & ) & 4) & $-0.025(5)$ \\
\hline$O(32 W)$ & ) & (7) & 0. & (5) & $0.023(5)$ & $-0.050(6)$ \\
\hline & & & & & $0.018(4)$ & 0.0 \\
\hline
\end{tabular}




\begin{tabular}{|c|c|c|c|c|c|c|}
\hline (35W) & & & 7) & ) & & \\
\hline$(36 W)$ & 03 & ) & 4) & .0 & 4) & -0 \\
\hline V) & & & 0) & & & \\
\hline V) & & & & & & \\
\hline ) & & & & & & 5) \\
\hline (40W) & 03 & 5) & 7) & $-0.009(5)$ & & (5) \\
\hline (41 & & & & & & \\
\hline & & & & & & \\
\hline $43 W)$ & & 027 & & (6) & & \\
\hline 1. & & & & & & \\
\hline$(45$ & ) & & 0.0 & & & \\
\hline 46 & 5) & ) & 0.0 & & & 4) \\
\hline$(47 W)$ & & & & & & \\
\hline $48 \mathrm{~W}$ & 4) & & & & & (3) \\
\hline 49 & & & & 4) & & $-c$ \\
\hline & & & & & & \\
\hline 51 & 8) & 5) & 0.02 & & & $5(6)$ \\
\hline$(52 W)$ & 9) & & & & & $-c$ \\
\hline (53) & & & & & & \\
\hline$(54$ & 5) & & & & & \\
\hline (55W) & 072 & 0.08 & 0.06 & -0.0 & (8) & (9) \\
\hline 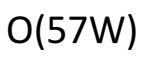 & 1 & & & & & \\
\hline$(58 W)$ & 5) & & & & & $3(6)$ \\
\hline (59W & 0 & & & & & (7) \\
\hline & & & & & & \\
\hline (60W) & 7) & 0.06 & 0.065 & -0.04 & $0.026(11)$ & $-0 . c$ \\
\hline (61W) & $.080(12)$ & $0.066(10)$ & $0.105(15)$ & $-0.048(1$ & 0.0 & -0.0 \\
\hline (63 & & & & & & \\
\hline $\mathrm{O}(63 \mathrm{~V})$ & .038 & 0.02 & 0.017 & (9) & .01 & $3(9)$ \\
\hline$(64 W)$ & $0.027(6)$ & $0.017(5)$ & $0.091(11)$ & $-0.020(6)$ & $-0.016(6)$ & $0.001(4)$ \\
\hline & 20 & & & $-0.09(2)$ & $-0.011(18)$ & $-0.041(1$ \\
\hline $\mathrm{O}(66 \mathrm{~W})$ & $0.12(3)$ & 0.08 & $0.02 \varepsilon$ & -0.02 & 0035 & -0.0 \\
\hline & & & & -0. & -0. & $-0.009(4)$ \\
\hline & $0.14(2)$ & $0.121(18)$ & $0.112(18)$ & $-0.099(16)$ & $0.075(16)$ & $-0.081(1$ \\
\hline & 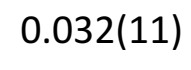 & 0.0 & 0.1 & -0 & $0 . c$ & -0.033 \\
\hline$(72 W)$ & $0.026(10)$ & $0.026(9)$ & $0.031(11)$ & $0.010(8)$ & $-0.015(8)$ & $0.000(8)$ \\
\hline
\end{tabular}




$\begin{array}{lllllll}\mathrm{O}(73 \mathrm{~W}) & 0.067(10) & 0.070(10) & 0.085(12) & -0.027(9) & 0.043(9) & -0.022(9) \\ \mathrm{O}(74 \mathrm{~W}) & 0.041(14) & 0.055(15) & 0.078(19) & -0.028(14) & 0.019(13) & -0.030(12) \\ \mathrm{O}(76 \mathrm{~W}) & 0.026(5) & 0.007(4) & 0.095(11) & 0.013(5) & 0.033(7) & 0.002(4) \\ \mathrm{O}(77 \mathrm{~W}) & 0.072(9) & 0.041(6) & 0.038(6) & -0.018(5) & 0.020(6) & -0.029(6)\end{array}$


Table S7: Bond lengths $[\AA ̊]$ for $\mathrm{Na} / \mathrm{K}\left\{\mathrm{U}_{24} \mathrm{Pp}_{12}\right\}$.

\begin{tabular}{|c|c|c|c|}
\hline atom-atom & distance & atom-atom & distance \\
\hline$U(1)-O(5)$ & $1.796(9)$ & $U(1)-O(3)$ & $1.800(9)$ \\
\hline$U(1)-O(1)$ & $2.330(10)$ & $U(1)-O(4)$ & $2.351(9)$ \\
\hline$U(1)-O(2)$ & $2.389(9)$ & $U(1)-O(6)$ & $2.395(10)$ \\
\hline $\mathrm{U}(1)-\mathrm{O}(8)$ & $2.421(10)$ & $\mathrm{U}(1)-\mathrm{O}(7) \# 1$ & $2.451(9)$ \\
\hline $\mathrm{U}(1)-\mathrm{Na}(1)$ & $4.030(13)$ & $U(2)-O(12)$ & $1.794(9)$ \\
\hline$U(2)-O(14)$ & $1.797(9)$ & $U(2)-O(13)$ & $2.331(9)$ \\
\hline$U(2)-O(10)$ & $2.350(9)$ & $U(2)-O(9)$ & $2.384(9)$ \\
\hline$U(2)-O(15)$ & $2.397(9)$ & $U(2)-O(42)$ & $2.407(8)$ \\
\hline$U(2)-O(11)$ & $2.443(9)$ & $U(2)-K(1)$ & $3.928(14)$ \\
\hline $\mathrm{U}(2)-\mathrm{Na}(11)$ & $3.935(15)$ & $\mathrm{U}(2)-\mathrm{Na}(2)$ & $4.062(13)$ \\
\hline$U(3)-O(43)$ & $1.804(9)$ & $\mathrm{U}(3)-\mathrm{O}(22)$ & $1.822(9)$ \\
\hline$U(3)-O(16)$ & $2.302(8)$ & $U(3)-O(18)$ & $2.311(9)$ \\
\hline$U(3)-O(19)$ & $2.384(9)$ & $U(3)-O(21)$ & $2.384(9)$ \\
\hline$U(3)-O(20) \# 1$ & $2.387(9)$ & $\mathrm{U}(3)-\mathrm{O}(17)$ & $2.410(9)$ \\
\hline $\mathrm{U}(3)-\mathrm{Na}(19)$ & $3.78(3)$ & $\mathrm{U}(3)-\mathrm{Na}(3)$ & $3.974(15)$ \\
\hline $\mathrm{U}(3)-\mathrm{Na}(1) \# 1$ & $4.149(15)$ & $U(4)-O(25)$ & $1.797(10)$ \\
\hline$U(4)-O(45)$ & $1.814(10)$ & $U(4)-O(18)$ & $2.302(9)$ \\
\hline$U(4)-O(24)$ & $2.309(8)$ & $\mathrm{U}(4)-\mathrm{O}(17)$ & $2.394(9)$ \\
\hline$U(4)-O(85)$ & $2.396(8)$ & $\mathrm{U}(4)-\mathrm{O}(23)$ & $2.399(9)$ \\
\hline$U(4)-O(44)$ & $2.421(9)$ & $U(4)-K(1)$ & $3.899(16)$ \\
\hline $\mathrm{U}(4)-\mathrm{Na}(3)$ & $4.022(17)$ & $\mathrm{U}(4)-\mathrm{Na}(11)$ & $4.089(16)$ \\
\hline$U(5)-O(29)$ & $1.784(9)$ & $U(5)-O(46)$ & $1.808(9)$ \\
\hline$U(5)-O(16)$ & $2.312(9)$ & $\mathrm{U}(5)-\mathrm{O}(26)$ & $2.326(10)$ \\
\hline $\mathrm{U}(5)-\mathrm{O}(28) \# 1$ & $2.381(9)$ & $U(5)-O(19)$ & $2.409(9)$ \\
\hline$U(5)-O(30)$ & $2.412(9)$ & $\mathrm{U}(5)-\mathrm{O}(27) \# 1$ & $2.416(9)$ \\
\hline$U(5)-K(2) \# 1$ & $3.926(14)$ & $\mathrm{U}(5)-\mathrm{Na}(3)$ & $3.950(14)$ \\
\hline$U(6)-O(47)$ & $1.806(9)$ & $U(6)-O(48)$ & $1.812(9)$ \\
\hline$U(6)-O(33)$ & $2.326(9)$ & $U(6)-O(1)$ & $2.337(10)$ \\
\hline $\mathrm{U}(6)-\mathrm{O}(8)$ & $2.393(10)$ & $U(6)-O(31)$ & $2.400(9)$ \\
\hline$U(6)-O(49)$ & $2.411(9)$ & $U(6)-O(32)$ & $2.424(10)$ \\
\hline$U(6)-K(3)$ & $4.044(16)$ & $\mathrm{U}(7)-\mathrm{O}(38)$ & $1.792(9)$ \\
\hline$U(7)-O(36)$ & $1.810(9)$ & $U(7)-O(26)$ & $2.312(9)$ \\
\hline$U(7)-O(24)$ & $2.326(9)$ & $\mathrm{U}(7)-\mathrm{O}(30)$ & $2.380(9)$ \\
\hline$U(7)-O(37)$ & $2.396(9)$ & $U(7)-O(34)$ & $2.408(9)$ \\
\hline$U(7)-O(85)$ & $2.411(9)$ & $U(7)-K(3)$ & $3.951(15)$ \\
\hline $\mathrm{U}(7)-\mathrm{Na}(7)$ & $4.103(16)$ & $\mathrm{U}(7)-\mathrm{Na}(3)$ & $4.108(14)$ \\
\hline$U(8)-O(40)$ & $1.813(10)$ & $U(8)-O(41)$ & $1.816(10)$ \\
\hline$U(8)-O(39)$ & $2.326(9)$ & $U(8)-O(13)$ & $2.353(9)$ \\
\hline$U(8)-O(86)$ & $2.392(10)$ & $U(8)-O(35)$ & $2.399(10)$ \\
\hline
\end{tabular}




\begin{tabular}{|c|c|c|c|}
\hline$U(8)-O(50)$ & $2.418(10)$ & $U(8)-O(15)$ & $2.437(10)$ \\
\hline$U(8)-K(1)$ & $4.141(17)$ & $U(8)-K(2)$ & $4.248(14)$ \\
\hline$U(9)-O(54)$ & $1.794(9)$ & $U(9)-O(56)$ & $1.808(9)$ \\
\hline$U(9)-O(52) \# 1$ & $2.314(9)$ & $\mathrm{U}(9)-\mathrm{O}(10) \# 1$ & $2.344(9)$ \\
\hline$U(9)-O(42) \# 1$ & $2.389(9)$ & $\mathrm{U}(9)-\mathrm{O}(55) \# 1$ & $2.390(10)$ \\
\hline$U(9)-O(51)$ & $2.412(9)$ & $U(9)-O(53)$ & $2.415(9)$ \\
\hline $\mathrm{U}(9)-\mathrm{Na}(11) \# 1$ & $3.951(15)$ & $U(10)-O(59)$ & $1.788(10)$ \\
\hline$U(10)-O(60)$ & $1.799(10)$ & $U(10)-O(39)$ & $2.345(9)$ \\
\hline$U(10)-O(52)$ & $2.347(9)$ & $U(10)-O(57)$ & $2.366(9)$ \\
\hline$U(10)-O(35)$ & $2.399(9)$ & $U(10)-O(55)$ & $2.411(8)$ \\
\hline$U(10)-O(58)$ & $2.425(9)$ & $\mathrm{U}(10)-\mathrm{Na}(13)$ & $3.945(16)$ \\
\hline$U(10)-K(2)$ & $3.961(14)$ & $U(11)-O(61)$ & $1.794(9)$ \\
\hline $\mathrm{U}(11)-\mathrm{O}(87)$ & $1.810(9)$ & $U(11)-O(4)$ & $2.324(9)$ \\
\hline$U(11)-O(63)$ & $2.340(10)$ & $U(11)-O(64)$ & $2.382(10)$ \\
\hline$U(11)-O(2)$ & $2.392(9)$ & $U(11)-O(65)$ & $2.392(11)$ \\
\hline$U(11)-O(62)$ & $2.425(9)$ & $\mathrm{U}(11)-\mathrm{Na}(7)$ & $4.033(14)$ \\
\hline$U(11)-\mathrm{Na}(16)$ & $4.133(5)$ & $U(12)-O(66)$ & $1.775(9)$ \\
\hline$U(12)-O(69)$ & $1.829(9)$ & $U(12)-O(33)$ & $2.345(9)$ \\
\hline$U(12)-O(63)$ & $2.347(10)$ & $U(12)-O(68)$ & $2.363(10)$ \\
\hline$U(12)-O(64)$ & $2.386(10)$ & $U(12)-O(67)$ & $2.386(9)$ \\
\hline$U(12)-O(49)$ & $2.413(9)$ & $U(12)-K(3)$ & $3.887(16)$ \\
\hline $\mathrm{U}(12)-\mathrm{Na}(7)$ & $4.020(14)$ & $\mathrm{I}(1)-\mathrm{O}(2 \mathrm{I})$ & $1.798(13)$ \\
\hline $\mathrm{I}(1)-\mathrm{O}(1 \mathrm{I})$ & $1.799(13)$ & $I(1)-O(3 I)$ & $1.804(14)$ \\
\hline $\mathrm{I}(1)-\mathrm{Na}(3)$ & $3.460(16)$ & $\mathrm{K}(1)-\mathrm{O}(4 \mathrm{~W})$ & $2.678(15)$ \\
\hline $\mathrm{K}(1)-\mathrm{O}(25)$ & $2.917(15)$ & $\mathrm{K}(1)-\mathrm{O}(50)$ & $2.930(18)$ \\
\hline $\mathrm{K}(1)-\mathrm{O}(85)$ & $2.968(18)$ & $\mathrm{K}(1)-\mathrm{O}(12)$ & $2.973(15)$ \\
\hline $\mathrm{K}(1)-\mathrm{H}(15 \mathrm{~A}) \# 2$ & $2.98(2)$ & $\mathrm{K}(1)-\mathrm{O}(11)$ & $3.009(16)$ \\
\hline $\mathrm{K}(1)-\mathrm{O}(76 \mathrm{~W})$ & $3.15(2)$ & $\mathrm{K}(1)-\mathrm{O}(23)$ & $3.275(17)$ \\
\hline $\mathrm{K}(1)-\mathrm{O}(41)$ & $3.327(18)$ & $\mathrm{K}(1)-\mathrm{O}(15 \mathrm{~W}) \# 2$ & $3.360(17)$ \\
\hline $\mathrm{K}(1)-\mathrm{O}(15)$ & $3.384(18)$ & $\mathrm{K}(2)-\mathrm{O}(5 \mathrm{~W})$ & $2.691(15)$ \\
\hline $\mathrm{K}(2)-\mathrm{O}(57)$ & $2.761(16)$ & $\mathrm{K}(2)-\mathrm{O}(29) \# 1$ & $2.905(16)$ \\
\hline $\mathrm{K}(2)-\mathrm{O}(31 \mathrm{~W}) \# 3$ & $2.90(2)$ & $\mathrm{K}(2)-\mathrm{O}(86)$ & $2.976(16)$ \\
\hline $\mathrm{K}(2)-\mathrm{H}(31 \mathrm{~B}) \# 3$ & $2.98(3)$ & $\mathrm{K}(2)-\mathrm{O}(19) \# 1$ & $2.990(15)$ \\
\hline $\mathrm{K}(2)-\mathrm{O}(33 \mathrm{~W})$ & $3.05(2)$ & $\mathrm{K}(2)-\mathrm{H}(33 \mathrm{~B})$ & $3.08(5)$ \\
\hline $\mathrm{K}(2)-\mathrm{H}(5 \mathrm{~B})$ & $3.09(2)$ & $\mathrm{K}(2)-\mathrm{O}(59)$ & $3.200(15)$ \\
\hline $\mathrm{K}(2)-\mathrm{O}(35)$ & $3.365(16)$ & $\mathrm{K}(3)-\mathrm{O}(7 \mathrm{~W})$ & $2.667(18)$ \\
\hline $\mathrm{K}(3)-\mathrm{H}(59 \mathrm{D})$ & $2.78(3)$ & $\mathrm{K}(3)-\mathrm{O}(68)$ & $2.781(18)$ \\
\hline $\mathrm{K}(3)-\mathrm{O}(77 \mathrm{~W})$ & $2.92(2)$ & $\mathrm{K}(3)-\mathrm{O}(36)$ & $2.951(18)$ \\
\hline $\mathrm{K}(3)-\mathrm{O}(32)$ & $3.028(17)$ & $K(3)-H(7 A)$ & $3.03(3)$ \\
\hline $\mathrm{K}(3)-\mathrm{O}(66)$ & $3.054(19)$ & $\mathrm{K}(3)-\mathrm{O}(30)$ & $3.067(17)$ \\
\hline $\mathrm{K}(3)-\mathrm{O}(59 \mathrm{~V})$ & $3.16(3)$ & $\mathrm{K}(3)-\mathrm{O}(47)$ & $3.203(18)$ \\
\hline $\mathrm{K}(3)-\mathrm{O}(49)$ & $3.239(18)$ & $\mathrm{Na}(1)-\mathrm{O}(33 \mathrm{~W})$ & $2.332(18)$ \\
\hline $\mathrm{Na}(1)-\mathrm{O}(5 \mathrm{~W})$ & $2.362(16)$ & $\mathrm{Na}(1)-\mathrm{O}(12 \mathrm{~W})$ & $2.406(17)$ \\
\hline $\mathrm{Na}(1)-\mathrm{O}(3)$ & $2.459(14)$ & $\mathrm{Na}(1)-\mathrm{O}(43) \# 1$ & $2.507(16)$ \\
\hline
\end{tabular}




\begin{tabular}{|c|c|c|c|}
\hline $\mathrm{Na}(1)-\mathrm{O}(39 \mathrm{~W})$ & $2.529(18)$ & $\mathrm{Na}(1)-\mathrm{O}(61)$ & $2.639(16)$ \\
\hline $\mathrm{Na}(1)-\mathrm{Na}(7)$ & $3.32(2)$ & $\mathrm{Na}(1)-\mathrm{U}(3) \# 1$ & $4.149(15)$ \\
\hline $\mathrm{Na}(2)-\mathrm{O}(10 \mathrm{~W})$ & $2.325(15)$ & $\mathrm{Na}(2)-\mathrm{O}(36 \mathrm{~W})$ & $2.362(16)$ \\
\hline $\mathrm{Na}(2)-\mathrm{O}(25 \mathrm{~W})$ & $2.402(17)$ & $\mathrm{Na}(2)-\mathrm{O}(14 \mathrm{~W})$ & $2.408(14)$ \\
\hline $\mathrm{Na}(2)-\mathrm{O}(14)$ & $2.424(16)$ & $\mathrm{Na}(2)-\mathrm{O}(1 \mathrm{~W}) \# 4$ & $2.431(16)$ \\
\hline $\mathrm{Na}(2)-\mathrm{Na}(9)$ & $3.516(18)$ & $\mathrm{Na}(2)-\mathrm{Na}(12) \# 4$ & $3.92(2)$ \\
\hline $\mathrm{Na}(3)-\mathrm{O}(62 \mathrm{~W})$ & $2.33(3)$ & $\mathrm{Na}(3)-\mathrm{O}(46)$ & $2.377(15)$ \\
\hline $\mathrm{Na}(3)-\mathrm{O}(3 \mathrm{I})$ & $2.398(16)$ & $\mathrm{Na}(3)-\mathrm{O}(22)$ & $2.425(15)$ \\
\hline $\mathrm{Na}(3)-\mathrm{O}(45)$ & $2.479(18)$ & $\mathrm{Na}(3)-\mathrm{O}(38)$ & $2.567(15)$ \\
\hline $\mathrm{Na}(4)-\mathrm{O}(77)$ & $2.347(13)$ & $\mathrm{Na}(4)-\mathrm{O}(20 \mathrm{~W})$ & $2.374(14)$ \\
\hline $\mathrm{Na}(4)-\mathrm{O}(27 \mathrm{~W})$ & $2.388(17)$ & $\mathrm{Na}(4)-\mathrm{O}(18 \mathrm{~W})$ & $2.395(15)$ \\
\hline $\mathrm{Na}(4)-\mathrm{O}(16 \mathrm{~W}) \# 5$ & $2.395(16)$ & $\mathrm{Na}(4)-\mathrm{O}(77) \# 5$ & $2.437(15)$ \\
\hline $\mathrm{Na}(4)-\mathrm{Na}(14) \# 5$ & $3.36(2)$ & $\mathrm{Na}(4)-\mathrm{Na}(4) \# 5$ & $3.46(2)$ \\
\hline $\mathrm{Na}(4)-\mathrm{Na}(19) \# 5$ & $3.56(2)$ & $\mathrm{Na}(4)-\mathrm{Na}(14)$ & $3.58(2)$ \\
\hline $\mathrm{Na}(5)-\mathrm{O}(2 \mathrm{I})$ & $2.337(18)$ & $\mathrm{Na}(5)-\mathrm{O}(9 \mathrm{~W}) \# 2$ & $2.359(15)$ \\
\hline $\mathrm{Na}(5)-\mathrm{O}(34 \mathrm{~W})$ & $2.430(17)$ & $\mathrm{Na}(5)-\mathrm{O}(43 \mathrm{~W})$ & $2.431(17)$ \\
\hline $\mathrm{Na}(5)-\mathrm{O}(76)$ & $2.433(16)$ & $\mathrm{Na}(5)-\mathrm{O}(15 \mathrm{~W})$ & $2.547(18)$ \\
\hline $\mathrm{Na}(5)-\mathrm{Na}(6) \# 2$ & $3.389(19)$ & $\mathrm{Na}(5)-\mathrm{Na}(6)$ & $3.48(2)$ \\
\hline $\mathrm{Na}(5)-\mathrm{Na}(20)$ & $3.58(3)$ & $\mathrm{Na}(5)-\mathrm{Na}(21)$ & $4.03(7)$ \\
\hline $\mathrm{Na}(6)-\mathrm{O}(9 \mathrm{~W})$ & $2.372(16)$ & $\mathrm{Na}(6)-\mathrm{O}(15 \mathrm{~W})$ & $2.376(17)$ \\
\hline $\mathrm{Na}(6)-\mathrm{O}(67 \mathrm{~W})$ & $2.398(16)$ & $\mathrm{Na}(6)-\mathrm{O}(76)$ & $2.417(15)$ \\
\hline $\mathrm{Na}(6)-\mathrm{O}(46 \mathrm{~W})$ & $2.444(17)$ & $\mathrm{Na}(6)-\mathrm{O}(76) \# 2$ & $2.480(14)$ \\
\hline $\mathrm{Na}(6)-\mathrm{Na}(5) \# 2$ & $3.389(19)$ & $\mathrm{Na}(6)-\mathrm{Na}(6) \# 2$ & $3.62(3)$ \\
\hline $\mathrm{Na}(7)-\mathrm{O}(4 \mathrm{~W})$ & $2.322(15)$ & $\mathrm{Na}(7)-\mathrm{O}(12 \mathrm{~W})$ & $2.404(16)$ \\
\hline $\mathrm{Na}(7)-\mathrm{O}(76 \mathrm{~W})$ & $2.430(15)$ & $\mathrm{Na}(7)-\mathrm{O}(66)$ & $2.495(15)$ \\
\hline $\mathrm{Na}(7)-\mathrm{O}(36)$ & $2.503(17)$ & $\mathrm{Na}(7)-\mathrm{O}(61)$ & $2.530(16)$ \\
\hline $\mathrm{Na}(7)-\mathrm{O}(39 \mathrm{~W})$ & $2.60(2)$ & $\mathrm{Na}(8)-\mathrm{O}(80) \# 6$ & $2.358(13)$ \\
\hline $\mathrm{Na}(8)-\mathrm{O}(24 \mathrm{~W})$ & $2.396(17)$ & $\mathrm{Na}(8)-\mathrm{O}(80)$ & $2.402(16)$ \\
\hline $\mathrm{Na}(8)-\mathrm{O}(35 \mathrm{~W})$ & $2.428(19)$ & $\mathrm{Na}(8)-\mathrm{O}(41 \mathrm{~W})$ & $2.437(18)$ \\
\hline $\mathrm{Na}(8)-\mathrm{O}(28 \mathrm{~W})$ & $2.506(15)$ & $\mathrm{Na}(8)-\mathrm{Na}(17) \# 6$ & $3.36(3)$ \\
\hline $\mathrm{Na}(8)-\mathrm{P}(12)$ & $3.410(16)$ & $\mathrm{Na}(8)-\mathrm{P}(12) \# 6$ & $3.445(14)$ \\
\hline $\mathrm{Na}(8)-\mathrm{Na}(17)$ & $3.48(2)$ & $\mathrm{Na}(8)-\mathrm{Na}(8) \# 6$ & $3.60(2)$ \\
\hline $\mathrm{Na}(9)-\mathrm{O}(14 \mathrm{~W})$ & $2.345(15)$ & $\mathrm{Na}(9)-\mathrm{O}(36 \mathrm{~W})$ & $2.345(16)$ \\
\hline $\mathrm{Na}(9)-\mathrm{O}(13 \mathrm{~W}) \# 7$ & $2.377(15)$ & $\mathrm{Na}(9)-\mathrm{O}(19 \mathrm{~W})$ & $2.392(17)$ \\
\hline $\mathrm{Na}(9)-\mathrm{O}(11 \mathrm{~W}) \# 7$ & $2.418(16)$ & $\mathrm{Na}(9)-\mathrm{O}(26 \mathrm{~W})$ & $2.425(19)$ \\
\hline $\mathrm{Na}(9)-\mathrm{Na}(13) \# 7$ & $3.593(19)$ & $\mathrm{Na}(10)-\mathrm{O}(8 \mathrm{~W})$ & $2.324(17)$ \\
\hline $\mathrm{Na}(10)-\mathrm{O}(31 \mathrm{~W})$ & $2.364(17)$ & $\mathrm{Na}(10)-\mathrm{O}(21 \mathrm{~W})$ & $2.408(18)$ \\
\hline $\mathrm{Na}(10)-\mathrm{O}(72)$ & $2.417(17)$ & $\mathrm{Na}(10)-\mathrm{O}(22 \mathrm{~W})$ & $2.499(18)$ \\
\hline $\mathrm{Na}(10)-\mathrm{O}(72) \# 3$ & $2.559(16)$ & $\mathrm{Na}(10)-\mathrm{Na}(15) \# 3$ & $3.44(2)$ \\
\hline $\mathrm{Na}(10)-\mathrm{Na}(15)$ & $3.453(19)$ & $\mathrm{Na}(10)-\mathrm{Na}(10) \# 3$ & $3.66(3)$ \\
\hline $\mathrm{Na}(10)-\mathrm{K}(2) \# 3$ & $4.50(2)$ & $\mathrm{Na}(11)-\mathrm{O}(32 \mathrm{~W})$ & $2.351(17)$ \\
\hline $\mathrm{Na}(11)-\mathrm{O}(6 \mathrm{~W})$ & $2.377(16)$ & $\mathrm{Na}(11)-\mathrm{O}(54) \# 1$ & $2.418(17)$ \\
\hline $\mathrm{Na}(11)-\mathrm{O}(12)$ & $2.422(17)$ & $\mathrm{Na}(11)-\mathrm{O}(64 \mathrm{~W})$ & $2.441(18)$ \\
\hline $\mathrm{Na}(11)-\mathrm{O}(25)$ & $2.474(18)$ & $\mathrm{Na}(11)-\mathrm{H}(64 \mathrm{~A})$ & $2.56(4)$ \\
\hline
\end{tabular}




\begin{tabular}{|c|c|c|c|}
\hline $\mathrm{Na}(11)-\mathrm{H}(32 \mathrm{C})$ & $2.60(4)$ & $\mathrm{Na}(11)-\mathrm{H}(64 \mathrm{D})$ & $2.60(3)$ \\
\hline $\mathrm{Na}(11)-\mathrm{H}(6 \mathrm{~B})$ & $2.66(2)$ & $\mathrm{Na}(11)-\mathrm{O}(61 \mathrm{~W}) \# 1$ & $2.92(3)$ \\
\hline $\mathrm{Na}(12)-\mathrm{O}(0 \mathrm{~W})$ & $2.309(17)$ & $\mathrm{Na}(12)-\mathrm{O}(3 \mathrm{~W})$ & $2.34(2)$ \\
\hline $\mathrm{Na}(12)-\mathrm{O}(1 \mathrm{~W})$ & $2.381(17)$ & $\mathrm{Na}(12)-\mathrm{O}(2 \mathrm{~W})$ & $2.393(17)$ \\
\hline $\mathrm{Na}(12)-\mathrm{O}(89)$ & $2.483(16)$ & $\mathrm{Na}(12)-\mathrm{O}(82)$ & $2.922(16)$ \\
\hline $\mathrm{Na}(12)-\mathrm{P}(5)$ & $3.336(16)$ & $\mathrm{Na}(12)-\mathrm{Na}(2) \# 8$ & $3.92(2)$ \\
\hline $\mathrm{Na}(13)-\mathrm{O}(60)$ & $2.318(17)$ & $\mathrm{Na}(13)-\mathrm{O}(30 \mathrm{~W})$ & $2.375(17)$ \\
\hline $\mathrm{Na}(13)-\mathrm{O}(13 \mathrm{~W})$ & $2.390(16)$ & $\mathrm{Na}(13)-\mathrm{O}(23 \mathrm{~W})$ & $2.398(17)$ \\
\hline $\mathrm{Na}(13)-\mathrm{O}(11 \mathrm{~W})$ & $2.419(18)$ & $\mathrm{Na}(13)-\mathrm{O}(29 \mathrm{~W})$ & $2.615(18)$ \\
\hline $\mathrm{Na}(13)-\mathrm{Na}(18)$ & $3.42(3)$ & $\mathrm{Na}(13)-\mathrm{Na}(9) \# 7$ & $3.593(19)$ \\
\hline $\mathrm{Na}(14)-\mathrm{O}(42 \mathrm{~W})$ & $2.34(2)$ & $\mathrm{Na}(14)-\mathrm{O}(69 \mathrm{~W})$ & $2.35(3)$ \\
\hline $\mathrm{Na}(14)-\mathrm{O}(16 \mathrm{~W})$ & $2.381(19)$ & $\mathrm{Na}(14)-\mathrm{O}(69 \mathrm{~V})$ & $2.42(3)$ \\
\hline $\mathrm{Na}(14)-\mathrm{O}(77)$ & $2.434(18)$ & $\mathrm{Na}(14)-\mathrm{O}(37 \mathrm{~W})$ & $2.44(2)$ \\
\hline $\mathrm{Na}(14)-\mathrm{O}(27 \mathrm{~W})$ & $2.88(2)$ & $\mathrm{Na}(14)-\mathrm{Na}(4) \# 5$ & $3.36(2)$ \\
\hline $\mathrm{Na}(14)-\mathrm{P}(11)$ & $3.448(17)$ & $\mathrm{Na}(15)-\mathrm{O}(72)$ & $2.326(15)$ \\
\hline $\mathrm{Na}(15)-\mathrm{O}(38 \mathrm{~W})$ & $2.376(18)$ & $\mathrm{Na}(15)-\mathrm{O}(8 \mathrm{~W})$ & $2.402(17)$ \\
\hline $\mathrm{Na}(15)-\mathrm{O}(17 \mathrm{~W})$ & $2.415(17)$ & $\mathrm{Na}(15)-\mathrm{O}(21 \mathrm{~W}) \# 3$ & $2.418(16)$ \\
\hline $\mathrm{Na}(15)-\mathrm{O}(29 \mathrm{~W})$ & $2.543(16)$ & $\mathrm{Na}(15)-\mathrm{Na}(18)$ & $3.19(3)$ \\
\hline $\mathrm{Na}(15)-\mathrm{Na}(10) \# 3$ & $3.44(2)$ & $\mathrm{Na}(15)-\mathrm{P}(2)$ & $3.440(16)$ \\
\hline $\mathrm{Na}(16)-\mathrm{O}(40 \mathrm{~W})$ & $2.363(15)$ & $\mathrm{Na}(16)-\mathrm{O}(40 \mathrm{~W}) \# 9$ & $2.363(15)$ \\
\hline $\mathrm{Na}(16)-\mathrm{O}(47 \mathrm{~W})$ & $2.392(12)$ & $\mathrm{Na}(16)-\mathrm{O}(47 \mathrm{~W}) \# 9$ & $2.392(12)$ \\
\hline $\mathrm{Na}(16)-\mathrm{O}(87)$ & $2.520(7)$ & $\mathrm{Na}(16)-\mathrm{O}(87) \# 9$ & $2.520(7)$ \\
\hline $\mathrm{Na}(16)-\mathrm{U}(11) \# 9$ & $4.133(5)$ & $\mathrm{Na}(17)-\mathrm{O}(50 \mathrm{~W})$ & $2.29(2)$ \\
\hline $\mathrm{Na}(17)-\mathrm{O}(24 \mathrm{~W}) \# 6$ & $2.32(2)$ & $\mathrm{Na}(17)-\mathrm{O}(77 \mathrm{~W})$ & $2.38(3)$ \\
\hline $\mathrm{Na}(17)-\mathrm{O}(80)$ & $2.40(2)$ & $\mathrm{Na}(17)-\mathrm{H}(41 \mathrm{~B})$ & $2.42(8)$ \\
\hline $\mathrm{Na}(17)-\mathrm{O}(49 \mathrm{~W})$ & $2.44(3)$ & $\mathrm{Na}(17)-\mathrm{O}(41 \mathrm{~W})$ & $2.46(3)$ \\
\hline $\mathrm{Na}(17)-\mathrm{H}(50 \mathrm{~B})$ & $2.59(3)$ & $\mathrm{Na}(17)-\mathrm{Na}(8) \# 6$ & $3.36(3)$ \\
\hline $\mathrm{Na}(17)-\mathrm{P}(12)$ & $3.40(3)$ & $\mathrm{Na}(18)-\mathrm{O}(17 \mathrm{~W})$ & $2.27(2)$ \\
\hline $\mathrm{Na}(18)-\mathrm{O}(30 \mathrm{~W})$ & $2.28(2)$ & $\mathrm{Na}(18)-\mathrm{O}(37 \mathrm{~W}) \# 10$ & $2.43(3)$ \\
\hline $\mathrm{Na}(18)-\mathrm{O}(38 \mathrm{~W})$ & $2.58(3)$ & $\mathrm{Na}(18)-\mathrm{H}(30 \mathrm{~B})$ & $2.60(3)$ \\
\hline $\mathrm{Na}(18)-\mathrm{O}(29 \mathrm{~W})$ & $2.67(3)$ & $\mathrm{Na}(19)-\mathrm{H}(20 \mathrm{~B}) \# 5$ & $2.08(6)$ \\
\hline $\mathrm{Na}(19)-\mathrm{O}(56 \mathrm{~W})$ & $2.25(3)$ & $\mathrm{Na}(19)-\mathrm{O}(6 \mathrm{~W})$ & $2.27(3)$ \\
\hline $\mathrm{Na}(19)-\mathrm{O}(17)$ & $2.33(2)$ & $\mathrm{Na}(19)-\mathrm{O}(20 \mathrm{~W}) \# 5$ & $2.44(3)$ \\
\hline $\mathrm{Na}(19)-\mathrm{O}(21)$ & $2.60(3)$ & $\mathrm{Na}(19)-\mathrm{H}(56 \mathrm{~B})$ & $2.61(4)$ \\
\hline $\mathrm{Na}(19)-\mathrm{O}(27 \mathrm{~W}) \# 5$ & $2.72(3)$ & $\mathrm{Na}(19)-\mathrm{P}(11)$ & $3.30(3)$ \\
\hline $\mathrm{Na}(19)-\mathrm{Na}(4) \# 5$ & $3.56(2)$ & $\mathrm{Na}(20)-\mathrm{Na}(21)$ & $1.26(8)$ \\
\hline $\mathrm{Na}(20)-\mathrm{O}(73 \mathrm{~W})$ & $2.31(3)$ & $\mathrm{Na}(20)-\mathrm{O}(70 \mathrm{~W})$ & $2.40(4)$ \\
\hline $\mathrm{Na}(20)-\mathrm{O}(60 \mathrm{~W})$ & $2.41(3)$ & $\mathrm{Na}(20)-\mathrm{O}(43 \mathrm{~W})$ & $2.50(4)$ \\
\hline $\mathrm{Na}(20)-\mathrm{O}(2 \mathrm{I})$ & $2.51(3)$ & $\mathrm{Na}(20)-\mathrm{H}(73 \mathrm{~B})$ & $2.66(10)$ \\
\hline $\mathrm{Na}(21)-\mathrm{O}(70 \mathrm{~W})$ & $1.63(7)$ & $\mathrm{Na}(21)-\mathrm{O}(60 \mathrm{~W})$ & $2.12(7)$ \\
\hline $\mathrm{Na}(21)-\mathrm{H}(70 \mathrm{~A})$ & $2.15(8)$ & $\mathrm{Na}(21)-\mathrm{H}(60 \mathrm{~A})$ & $2.31(8)$ \\
\hline $\mathrm{Na}(21)-\mathrm{H}(70 \mathrm{~B})$ & $2.43(10)$ & $\mathrm{Na}(21)-\mathrm{O}(2 \mathrm{I})$ & $2.71(7)$ \\
\hline$P(1)-O(9)$ & $1.493(11)$ & $P(1)-O(70)$ & $1.508(11)$ \\
\hline$P(1)-O(44)$ & $1.526(12)$ & $P(1)-O(71)$ & $1.629(11)$ \\
\hline
\end{tabular}




\begin{tabular}{|c|c|c|c|}
\hline$P(2)-O(72)$ & $1.487(11)$ & $P(2)-O(57)$ & $1.520(12)$ \\
\hline$P(2)-O(27)$ & $1.537(11)$ & $P(2)-O(73)$ & $1.628(11)$ \\
\hline$P(3)-O(88)$ & $1.494(13)$ & $P(3)-O(62)$ & $1.520(12)$ \\
\hline$P(3)-O(50)$ & $1.529(12)$ & $P(3)-O(84)$ & $1.608(14)$ \\
\hline$P(4)-O(75)$ & $1.509(12)$ & $\mathrm{P}(4)-\mathrm{O}(6)$ & $1.511(12)$ \\
\hline$P(4)-O(20)$ & $1.533(11)$ & $\mathrm{P}(4)-\mathrm{O}(74) \# 1$ & $1.614(11)$ \\
\hline$P(5)-O(89)$ & $1.491(12)$ & $P(5)-O(32)$ & $1.524(12)$ \\
\hline$P(5)-O(51)$ & $1.533(11)$ & $P(5)-O(82)$ & $1.630(11)$ \\
\hline$P(6)-O(90)$ & $1.465(13)$ & $P(6)-O(86)$ & $1.511(11)$ \\
\hline$P(6)-O(65)$ & $1.538(12)$ & $P(6)-O(84)$ & $1.608(12)$ \\
\hline $\mathrm{P}(7)-\mathrm{O}(76)$ & $1.486(11)$ & $P(7)-O(11)$ & $1.528(12)$ \\
\hline$P(7)-O(23)$ & $1.528(12)$ & $\mathrm{P}(7)-\mathrm{O}(71)$ & $1.615(10)$ \\
\hline$P(8)-O(81)$ & $1.498(11)$ & $\mathrm{P}(8)-\mathrm{O}(28)$ & $1.526(12)$ \\
\hline$P(8)-O(58)$ & $1.533(11)$ & $P(8)-O(73)$ & $1.621(11)$ \\
\hline$P(9)-O(78)$ & $1.508(11)$ & $P(9)-O(67)$ & $1.513(12)$ \\
\hline$P(9)-O(34)$ & $1.536(11)$ & $P(9)-O(79)$ & $1.615(12)$ \\
\hline $\mathrm{P}(10)-\mathrm{O}(83)$ & $1.506(10)$ & $\mathrm{P}(10)-\mathrm{O}(31)$ & $1.510(11)$ \\
\hline$P(10)-O(53)$ & $1.534(10)$ & $\mathrm{P}(10)-\mathrm{O}(82)$ & $1.612(12)$ \\
\hline $\mathrm{P}(11)-\mathrm{O}(77)$ & $1.493(10)$ & $\mathrm{P}(11)-\mathrm{O}(21)$ & $1.517(11)$ \\
\hline$P(11)-O(7)$ & $1.532(12)$ & $\mathrm{P}(11)-\mathrm{O}(74)$ & $1.640(12)$ \\
\hline$P(12)-O(80)$ & $1.484(12)$ & $P(12)-O(68)$ & $1.515(11)$ \\
\hline $\mathrm{P}(12)-\mathrm{O}(37)$ & $1.541(10)$ & $\mathrm{P}(12)-\mathrm{O}(79)$ & $1.616(11)$ \\
\hline $\mathrm{P}(12)-\mathrm{Na}(8) \# 6$ & $3.445(14)$ & $\mathrm{O}(1)-\mathrm{O}(8)$ & $1.458(11)$ \\
\hline $\mathrm{O}(2)-\mathrm{O}(4)$ & $1.460(12)$ & $\mathrm{O}(7)-U(1) \# 1$ & $2.451(9)$ \\
\hline $\mathrm{O}(10)-\mathrm{O}(42)$ & $1.472(11)$ & $\mathrm{O}(10)-\mathrm{U}(9) \# 1$ & $2.344(9)$ \\
\hline $\mathrm{O}(13)-\mathrm{O}(15)$ & $1.475(11)$ & $\mathrm{O}(16)-\mathrm{O}(19)$ & $1.466(11)$ \\
\hline $\mathrm{O}(17)-\mathrm{O}(18)$ & $1.470(10)$ & $\mathrm{O}(19)-\mathrm{K}(2) \# 1$ & $2.990(15)$ \\
\hline$O(20)-U(3) \# 1$ & $2.387(9)$ & $\mathrm{O}(24)-\mathrm{O}(85)$ & $1.480(11)$ \\
\hline $\mathrm{O}(26)-\mathrm{O}(30)$ & $1.473(11)$ & $\mathrm{O}(27)-\mathrm{U}(5) \# 1$ & $2.416(9)$ \\
\hline $\mathrm{O}(28)-\mathrm{U}(5) \# 1$ & $2.381(9)$ & O(29)-К(2)\#1 & $2.905(16)$ \\
\hline $\mathrm{O}(33)-\mathrm{O}(49)$ & $1.459(12)$ & $\mathrm{O}(35)-\mathrm{O}(39)$ & $1.464(10)$ \\
\hline $\mathrm{O}(42)-\mathrm{U}(9) \# 1$ & $2.389(9)$ & $\mathrm{O}(43)-\mathrm{Na}(1) \# 1$ & $2.508(16)$ \\
\hline $\mathrm{O}(52)-\mathrm{O}(55)$ & $1.474(10)$ & $\mathrm{O}(52)-\mathrm{U}(9) \# 1$ & $2.314(9)$ \\
\hline $\mathrm{O}(54)-\mathrm{Na}(11) \# 1$ & $2.418(17)$ & $\mathrm{O}(55)-\mathrm{U}(9) \# 1$ & $2.390(10)$ \\
\hline $\mathrm{O}(63)-\mathrm{O}(64)$ & $1.474(11)$ & $\mathrm{O}(72)-\mathrm{Na}(10) \# 3$ & $2.559(16)$ \\
\hline $\mathrm{O}(74)-\mathrm{P}(4) \# 1$ & $1.614(11)$ & $\mathrm{O}(76)-\mathrm{Na}(6) \# 2$ & $2.480(14)$ \\
\hline $\mathrm{O}(77)-\mathrm{Na}(4) \# 5$ & $2.437(15)$ & $\mathrm{O}(80)-\mathrm{Na}(8) \# 6$ & $2.358(13)$ \\
\hline $\mathrm{O}(\mathrm{OW})-\mathrm{H}(\mathrm{OB})$ & $0.90(3)$ & $\mathrm{O}(\mathrm{OW})-\mathrm{H}(\mathrm{OA})$ & $0.96(2)$ \\
\hline $\mathrm{O}(1 \mathrm{~W})-\mathrm{H}(1 \mathrm{~B})$ & $0.94(2)$ & $\mathrm{O}(1 \mathrm{~W})-\mathrm{H}(1 \mathrm{~A})$ & $0.95(2)$ \\
\hline $\mathrm{O}(1 \mathrm{~W})-\mathrm{Na}(2) \# 8$ & $2.431(16)$ & $\mathrm{O}(2 \mathrm{~W})-\mathrm{H}(2 \mathrm{~A})$ & $0.93(3)$ \\
\hline $\mathrm{O}(2 \mathrm{~W})-\mathrm{H}(2 \mathrm{~B})$ & $0.95(4)$ & $\mathrm{O}(3 \mathrm{~W})-\mathrm{H}(3 \mathrm{~B})$ & $0.92(5)$ \\
\hline $\mathrm{O}(3 \mathrm{~W})-\mathrm{H}(3 \mathrm{~A})$ & $0.95(4)$ & $\mathrm{O}(4 \mathrm{~W})-\mathrm{H}(4 \mathrm{~A})$ & $0.98(2)$ \\
\hline $\mathrm{O}(4 \mathrm{~W})-\mathrm{H}(4 \mathrm{~B})$ & $0.99(2)$ & $\mathrm{O}(5 \mathrm{~W})-\mathrm{H}(5 \mathrm{~A})$ & $0.94(2)$ \\
\hline $\mathrm{O}(5 \mathrm{~W})-\mathrm{H}(5 \mathrm{~B})$ & $0.97(2)$ & $\mathrm{O}(6 \mathrm{~W})-\mathrm{H}(6 \mathrm{~B})$ & $0.96(3)$ \\
\hline
\end{tabular}




\begin{tabular}{|c|c|c|c|}
\hline $\mathrm{O}(6 \mathrm{~W})-\mathrm{H}(6 \mathrm{~A})$ & $1.00(2)$ & $\mathrm{O}(7 \mathrm{~W})-\mathrm{H}(7 \mathrm{~B})$ & $0.95(4)$ \\
\hline $\mathrm{O}(7 \mathrm{~W})-\mathrm{H}(7 \mathrm{~A})$ & $0.96(2)$ & $\mathrm{O}(8 \mathrm{~W})-\mathrm{H}(8 \mathrm{~B})$ & $0.94(3)$ \\
\hline $\mathrm{O}(8 \mathrm{~W})-\mathrm{H}(8 \mathrm{~A})$ & $0.976(19)$ & $\mathrm{O}(9 \mathrm{~W})-\mathrm{H}(9 \mathrm{~B})$ & $0.951(10)$ \\
\hline $\mathrm{O}(9 \mathrm{~W})-\mathrm{H}(9 \mathrm{~A})$ & $0.99(2)$ & $\mathrm{O}(9 \mathrm{~W})-\mathrm{Na}(5) \# 2$ & $2.359(15)$ \\
\hline $\mathrm{O}(10 \mathrm{~W})-\mathrm{H}(10 \mathrm{~B})$ & $0.97(2)$ & $\mathrm{O}(10 \mathrm{~W})-\mathrm{H}(10 \mathrm{~A})$ & $0.98(2)$ \\
\hline $\mathrm{O}(11 \mathrm{~W})-\mathrm{H}(11 \mathrm{~A})$ & $0.955(10)$ & $\mathrm{O}(11 \mathrm{~W})-\mathrm{H}(11 \mathrm{~B})$ & $0.99(2)$ \\
\hline $\mathrm{O}(11 \mathrm{~W})-\mathrm{Na}(9) \# 7$ & $2.418(16)$ & $\mathrm{O}(12 \mathrm{~W})-\mathrm{H}(12 \mathrm{~A})$ & $0.95(2)$ \\
\hline $\mathrm{O}(12 \mathrm{~W})-\mathrm{H}(12 \mathrm{~B})$ & $0.97(2)$ & $\mathrm{O}(13 \mathrm{~W})-\mathrm{H}(13 \mathrm{~A})$ & $0.95(2)$ \\
\hline $\mathrm{O}(13 \mathrm{~W})-\mathrm{H}(13 \mathrm{~B})$ & $0.954(10)$ & $\mathrm{O}(13 \mathrm{~W})-\mathrm{Na}(9) \# 7$ & $2.377(15)$ \\
\hline $\mathrm{O}(14 \mathrm{~W})-\mathrm{H}(14 \mathrm{~B})$ & $0.95(3)$ & $\mathrm{O}(14 \mathrm{~W})-\mathrm{H}(14 \mathrm{~A})$ & $0.96(2)$ \\
\hline $\mathrm{O}(15 \mathrm{~W})-\mathrm{H}(15 \mathrm{~B})$ & $0.955(10)$ & $\mathrm{O}(15 \mathrm{~W})-\mathrm{H}(15 \mathrm{~A})$ & $0.97(2)$ \\
\hline $\mathrm{O}(15 \mathrm{~W})-\mathrm{K}(1) \# 2$ & $3.360(17)$ & $H(15 A)-K(1) \# 2$ & $2.98(2)$ \\
\hline $\mathrm{O}(16 \mathrm{~W})-\mathrm{H}(16 \mathrm{~B})$ & $0.954(10)$ & $\mathrm{O}(16 \mathrm{~W})-\mathrm{H}(16 \mathrm{~A})$ & $0.97(3)$ \\
\hline $\mathrm{O}(16 \mathrm{~W})-\mathrm{Na}(4) \# 5$ & $2.395(16)$ & $\mathrm{O}(17 \mathrm{~W})-\mathrm{H}(17 \mathrm{~B})$ & $0.967(11)$ \\
\hline $\mathrm{O}(17 \mathrm{~W})-\mathrm{H}(17 \mathrm{~A})$ & $0.98(4)$ & $\mathrm{O}(18 \mathrm{~W})-\mathrm{H}(18 \mathrm{~A})$ & $0.95(2)$ \\
\hline $\mathrm{O}(18 \mathrm{~W})-\mathrm{H}(18 \mathrm{~B})$ & $0.96(3)$ & $\mathrm{O}(19 \mathrm{~W})-\mathrm{H}(19 \mathrm{~B})$ & $0.98(4)$ \\
\hline $\mathrm{O}(19 \mathrm{~W})-\mathrm{H}(19 \mathrm{~A})$ & $0.99(2)$ & $\mathrm{O}(20 \mathrm{~W})-\mathrm{H}(20 \mathrm{~B})$ & $0.963(11)$ \\
\hline $\mathrm{O}(20 \mathrm{~W})-\mathrm{H}(20 \mathrm{C})$ & $0.97(5)$ & $\mathrm{O}(20 \mathrm{~W})-\mathrm{H}(20 \mathrm{~A})$ & $0.98(2)$ \\
\hline $\mathrm{O}(20 \mathrm{~W})-\mathrm{Na}(19) \# 5$ & $2.44(3)$ & $H(20 B)-H(20 C)$ & $0.64(5)$ \\
\hline $\mathrm{H}(20 \mathrm{~B})-\mathrm{Na}(19) \# 5$ & $2.08(6)$ & $H(20 C)-H(20 B)$ & $0.64(5)$ \\
\hline $\mathrm{O}(21 \mathrm{~W})-\mathrm{H}(21 \mathrm{~A})$ & $0.96(3)$ & $\mathrm{O}(21 \mathrm{~W})-\mathrm{H}(21 \mathrm{~B})$ & $0.98(4)$ \\
\hline $\mathrm{O}(21 \mathrm{~W})-\mathrm{Na}(15) \# 3$ & $2.418(16)$ & $\mathrm{O}(22 \mathrm{~W})-\mathrm{H}(22 \mathrm{~B})$ & $0.94(4)$ \\
\hline $\mathrm{O}(22 \mathrm{~W})-\mathrm{H}(22 \mathrm{~A})$ & $0.98(3)$ & $\mathrm{O}(23 \mathrm{~W})-\mathrm{H}(23 \mathrm{~B})$ & $0.94(2)$ \\
\hline $\mathrm{O}(23 \mathrm{~W})-\mathrm{H}(23 \mathrm{~A})$ & $0.97(2)$ & $\mathrm{O}(24 \mathrm{~W})-\mathrm{H}(24 \mathrm{~B})$ & $0.955(10)$ \\
\hline $\mathrm{O}(24 \mathrm{~W})-\mathrm{H}(24 \mathrm{~A})$ & $0.97(2)$ & $\mathrm{O}(24 \mathrm{~W})-\mathrm{Na}(17) \# 6$ & $2.32(2)$ \\
\hline $\mathrm{O}(25 \mathrm{~W})-\mathrm{H}(25 \mathrm{~B})$ & $0.96(2)$ & $\mathrm{O}(25 \mathrm{~W})-\mathrm{H}(25 \mathrm{~A})$ & $0.961(11)$ \\
\hline $\mathrm{O}(26 \mathrm{~W})-\mathrm{H}(26 \mathrm{~B})$ & $0.960(11)$ & $\mathrm{O}(26 \mathrm{~W})-\mathrm{H}(26 \mathrm{~A})$ & $0.98(3)$ \\
\hline $\mathrm{O}(27 \mathrm{~W})-\mathrm{H}(27 \mathrm{~B})$ & $0.957(10)$ & $\mathrm{O}(27 \mathrm{~W})-\mathrm{H}(27 \mathrm{~A})$ & $0.958(10)$ \\
\hline $\mathrm{O}(27 \mathrm{~W})-\mathrm{Na}(19) \# 5$ & $2.72(3)$ & $\mathrm{O}(28 \mathrm{~W})-\mathrm{H}(28 \mathrm{~B})$ & $0.94(3)$ \\
\hline $\mathrm{O}(28 \mathrm{~W})-\mathrm{H}(28 \mathrm{~A})$ & $0.956(10)$ & $\mathrm{O}(29 W)-\mathrm{H}(29 A)$ & $0.94(2)$ \\
\hline $\mathrm{O}(29 \mathrm{~W})-\mathrm{H}(29 \mathrm{~B})$ & $0.97(3)$ & $\mathrm{O}(30 \mathrm{~W})-\mathrm{H}(30 \mathrm{~A})$ & $0.95(2)$ \\
\hline $\mathrm{O}(30 \mathrm{~W})-\mathrm{H}(30 \mathrm{~B})$ & $0.98(3)$ & $\mathrm{O}(31 \mathrm{~W})-\mathrm{H}(31 \mathrm{~B})$ & $0.955(11)$ \\
\hline $\mathrm{O}(31 \mathrm{~W})-\mathrm{H}(31 \mathrm{~A})$ & $0.957(10)$ & $\mathrm{O}(31 W)-K(2) \# 3$ & $2.90(2)$ \\
\hline$H(31 B)-K(2) \# 3$ & $2.98(3)$ & $\mathrm{O}(32 \mathrm{~W})-\mathrm{H}(32 \mathrm{~A})$ & $0.949(10)$ \\
\hline $\mathrm{O}(32 \mathrm{~W})-\mathrm{H}(32 \mathrm{C})$ & $0.956(11)$ & $\mathrm{O}(32 \mathrm{~W})-\mathrm{H}(32 \mathrm{~B})$ & $0.959(11)$ \\
\hline$H(32 B)-H(32 C)$ & $1.01(4)$ & $H(32 C)-H(32 B)$ & $1.01(4)$ \\
\hline $\mathrm{O}(33 \mathrm{~W})-\mathrm{H}(33 \mathrm{~B})$ & $0.950(11)$ & $\mathrm{O}(33 \mathrm{~W})-\mathrm{H}(33 \mathrm{~A})$ & $0.953(10)$ \\
\hline $\mathrm{O}(33 \mathrm{~W})-\mathrm{H}(33 \mathrm{C})$ & $0.954(11)$ & $H(33 B)-H(33 C)$ & $0.56(6)$ \\
\hline$H(33 C)-H(33 B)$ & $0.56(6)$ & $\mathrm{O}(34 \mathrm{~W})-\mathrm{H}(34 \mathrm{~B})$ & $0.952(11)$ \\
\hline $\mathrm{O}(34 \mathrm{~W})-\mathrm{H}(34 \mathrm{~A})$ & $0.955(10)$ & $\mathrm{O}(35 \mathrm{~W})-\mathrm{H}(35 \mathrm{~B})$ & $0.95(3)$ \\
\hline $\mathrm{O}(35 \mathrm{~W})-\mathrm{H}(35 \mathrm{~A})$ & $0.95(3)$ & $\mathrm{O}(36 \mathrm{~W})-\mathrm{H}(36 \mathrm{~B})$ & $0.955(10)$ \\
\hline $\mathrm{O}(36 \mathrm{~W})-\mathrm{H}(36 \mathrm{~A})$ & $0.962(11)$ & $\mathrm{O}(37 \mathrm{~W})-\mathrm{H}(37 \mathrm{~A})$ & $0.960(10)$ \\
\hline $\mathrm{O}(37 \mathrm{~W})-\mathrm{H}(37 \mathrm{~B})$ & $0.963(11)$ & $\mathrm{O}(37 \mathrm{~W})-\mathrm{Na}(18) \# 11$ & $2.43(3)$ \\
\hline $\mathrm{O}(38 \mathrm{~W})-\mathrm{H}(38 \mathrm{~B})$ & $0.956(11)$ & $\mathrm{O}(38 \mathrm{~W})-\mathrm{H}(38 \mathrm{C})$ & $0.959(11)$ \\
\hline
\end{tabular}




\begin{tabular}{|c|c|c|c|}
\hline $\mathrm{O}(38 \mathrm{~W})-\mathrm{H}(38 \mathrm{~A})$ & $0.962(11)$ & $H(38 \mathrm{~A})-\mathrm{H}(38 \mathrm{C})$ & $0.59(5)$ \\
\hline$H(38 C)-H(38 A)$ & $0.59(5)$ & $\mathrm{O}(39 \mathrm{~W})-\mathrm{H}(39 \mathrm{~A})$ & $0.956(10)$ \\
\hline $\mathrm{O}(39 \mathrm{~W})-\mathrm{H}(39 \mathrm{~B})$ & $0.963(11)$ & $\mathrm{O}(40 \mathrm{~W})-\mathrm{H}(40 \mathrm{~B})$ & $0.959(11)$ \\
\hline $\mathrm{O}(40 \mathrm{~W})-\mathrm{H}(40 \mathrm{~A})$ & $0.960(10)$ & $\mathrm{O}(41 \mathrm{~W})-\mathrm{H}(41 \mathrm{C})$ & $0.957(11)$ \\
\hline $\mathrm{O}(41 \mathrm{~W})-\mathrm{H}(41 \mathrm{~B})$ & $0.958(11)$ & $\mathrm{O}(41 \mathrm{~W})-\mathrm{H}(41 \mathrm{~A})$ & $0.963(11)$ \\
\hline $\mathrm{H}(41 \mathrm{~B})-\mathrm{H}(41 \mathrm{C})$ & $1.00(9)$ & $H(41 C)-H(41 B)$ & $1.00(9)$ \\
\hline $\mathrm{O}(42 \mathrm{~W})-\mathrm{H}(42 \mathrm{~A})$ & $0.959(11)$ & $\mathrm{O}(42 \mathrm{~W})-\mathrm{H}(42 \mathrm{~B})$ & $0.961(11)$ \\
\hline $\mathrm{O}(43 \mathrm{~W})-\mathrm{H}(43 \mathrm{~B})$ & $0.957(11)$ & $\mathrm{O}(43 \mathrm{~W})-\mathrm{H}(43 \mathrm{~A})$ & $0.962(11)$ \\
\hline $\mathrm{O}(44 \mathrm{~W})-\mathrm{H}(44 \mathrm{~B})$ & $0.957(11)$ & $\mathrm{O}(44 \mathrm{~W})-\mathrm{H}(44 \mathrm{~A})$ & $0.96(3)$ \\
\hline $\mathrm{O}(45 \mathrm{~W})-\mathrm{H}(45 \mathrm{~A})$ & $0.958(11)$ & $\mathrm{O}(45 \mathrm{~W})-\mathrm{H}(45 \mathrm{~B})$ & $0.960(11)$ \\
\hline $\mathrm{O}(46 \mathrm{~W})-\mathrm{H}(46 \mathrm{~A})$ & $0.96(2)$ & $\mathrm{O}(46 \mathrm{~W})-\mathrm{H}(46 \mathrm{~B})$ & $0.959(19)$ \\
\hline $\mathrm{O}(47 \mathrm{~W})-\mathrm{H}(47 \mathrm{~A})$ & $0.957(10)$ & $\mathrm{O}(47 \mathrm{~W})-\mathrm{H}(47 \mathrm{~B})$ & $0.958(11)$ \\
\hline $\mathrm{O}(47 \mathrm{~W})-\mathrm{H}(47 \mathrm{C})$ & $0.959(11)$ & $\mathrm{O}(48 \mathrm{~W})-\mathrm{H}(48 \mathrm{~A})$ & $0.95(2)$ \\
\hline $\mathrm{O}(48 \mathrm{~W})-\mathrm{H}(48 \mathrm{~B})$ & $0.96(2)$ & $\mathrm{O}(49 \mathrm{~W})-\mathrm{H}(49 \mathrm{~A})$ & $0.953(10)$ \\
\hline $\mathrm{O}(49 \mathrm{~W})-\mathrm{H}(49 \mathrm{~B})$ & $0.957(10)$ & $\mathrm{O}(50 \mathrm{~W})-\mathrm{H}(50 \mathrm{~B})$ & $0.96(3)$ \\
\hline $\mathrm{O}(50 \mathrm{~W})-\mathrm{H}(50 \mathrm{~A})$ & $0.959(10)$ & $\mathrm{O}(51 \mathrm{~W})-\mathrm{H}(51 \mathrm{~B})$ & $0.965(11)$ \\
\hline $\mathrm{O}(51 \mathrm{~W})-\mathrm{H}(51 \mathrm{~A})$ & $0.98(3)$ & $\mathrm{O}(52 \mathrm{~W})-\mathrm{H}(52 \mathrm{~B})$ & $0.95(4)$ \\
\hline $\mathrm{O}(52 \mathrm{~W})-\mathrm{H}(52 \mathrm{~A})$ & $0.956(11)$ & $\mathrm{O}(53 \mathrm{~W})-\mathrm{H}(53 \mathrm{~A})$ & $0.960(11)$ \\
\hline $\mathrm{O}(53 \mathrm{~W})-\mathrm{H}(53 \mathrm{~B})$ & $0.960(11)$ & $\mathrm{O}(54 \mathrm{~W})-\mathrm{H}(54 \mathrm{~A})$ & $0.98(3)$ \\
\hline $\mathrm{O}(54 \mathrm{~W})-\mathrm{H}(54 \mathrm{~B})$ & $1.00(3)$ & $\mathrm{O}(55 \mathrm{~W})-\mathrm{H}(55 \mathrm{~B})$ & $0.954(11)$ \\
\hline $\mathrm{O}(55 \mathrm{~W})-\mathrm{H}(55 \mathrm{~A})$ & $0.956(11)$ & $\mathrm{O}(56 \mathrm{~W})-\mathrm{H}(56 \mathrm{~B})$ & $0.960(11)$ \\
\hline $\mathrm{O}(56 \mathrm{~W})-\mathrm{H}(56 \mathrm{~A})$ & $0.960(11)$ & $\mathrm{O}(57 \mathrm{~W})-\mathrm{H}(57 \mathrm{~B})$ & $0.957(11)$ \\
\hline $\mathrm{O}(57 \mathrm{~W})-\mathrm{H}(57 \mathrm{~A})$ & $0.958(11)$ & $\mathrm{O}(58 \mathrm{~W})-\mathrm{H}(58 \mathrm{~A})$ & $0.956(11)$ \\
\hline $\mathrm{O}(58 \mathrm{~W})-\mathrm{H}(58 \mathrm{~B})$ & $0.956(11)$ & $\mathrm{O}(59 \mathrm{~W})-\mathrm{H}(59 \mathrm{C})$ & $0.76(5)$ \\
\hline $\mathrm{O}(59 \mathrm{~W})-\mathrm{O}(59 \mathrm{~V})$ & $0.801(19)$ & $\mathrm{O}(59 \mathrm{~W})-\mathrm{H}(59 \mathrm{~B})$ & $0.961(11)$ \\
\hline $\mathrm{O}(59 \mathrm{~W})-\mathrm{H}(59 \mathrm{~A})$ & $0.962(11)$ & $\mathrm{O}(59 \mathrm{~V})-\mathrm{H}(59 \mathrm{C})$ & $0.962(11)$ \\
\hline $\mathrm{O}(59 \mathrm{~V})-\mathrm{H}(59 \mathrm{D})$ & $0.963(11)$ & $\mathrm{O}(59 \mathrm{~V})-\mathrm{H}(59 \mathrm{~A})$ & $1.45(3)$ \\
\hline$H(59 A)-H(59 C)$ & $0.53(4)$ & $H(59 C)-H(59 A)$ & $0.53(4)$ \\
\hline $\mathrm{O}(60 \mathrm{~W})-\mathrm{H}(60 \mathrm{~B})$ & $0.955(11)$ & $\mathrm{O}(60 \mathrm{~W})-\mathrm{H}(60 \mathrm{~A})$ & $0.958(11)$ \\
\hline $\mathrm{O}(61 \mathrm{~W})-\mathrm{H}(61 \mathrm{~B})$ & $0.954(11)$ & $\mathrm{O}(61 \mathrm{~W})-\mathrm{H}(61 \mathrm{~A})$ & $0.957(11)$ \\
\hline $\mathrm{O}(61 \mathrm{~W})-\mathrm{Na}(11) \# 1$ & $2.92(3)$ & $\mathrm{O}(62 \mathrm{~W})-\mathrm{H}(62 \mathrm{~B})$ & $0.961(11)$ \\
\hline $\mathrm{O}(62 \mathrm{~W})-\mathrm{H}(62 \mathrm{~A})$ & $0.965(11)$ & $H(62 A)-H(62 B)$ & $0.97(7)$ \\
\hline$H(62 B)-H(62 A)$ & $0.97(7)$ & $O(63 W)-O(63 V)$ & $0.78(3)$ \\
\hline $\mathrm{O}(63 \mathrm{~W})-\mathrm{H}(63 \mathrm{~A})$ & $0.965(11)$ & $\mathrm{O}(63 \mathrm{~W})-\mathrm{H}(63 \mathrm{~B})$ & $0.965(11)$ \\
\hline $\mathrm{O}(63 \mathrm{~W})-\mathrm{H}(63 \mathrm{C})$ & $1.06(6)$ & $\mathrm{O}(63 \mathrm{~V})-\mathrm{H}(63 \mathrm{~B})$ & $0.94(6)$ \\
\hline $\mathrm{O}(63 \mathrm{~V})-\mathrm{H}(63 \mathrm{~A})$ & $1.04(4)$ & $\mathrm{O}(64 \mathrm{~W})-\mathrm{H}(64 \mathrm{D})$ & $0.969(11)$ \\
\hline $\mathrm{O}(64 \mathrm{~W})-\mathrm{H}(64 \mathrm{~A})$ & $0.971(11)$ & $\mathrm{O}(64 \mathrm{~W})-\mathrm{H}(64 \mathrm{~B})$ & $0.968(11)$ \\
\hline $\mathrm{O}(64 \mathrm{~W})-\mathrm{H}(64 \mathrm{C})$ & $0.972(11)$ & $H(64 B)-H(64 D)$ & $0.96(4)$ \\
\hline$H(64 D)-H(64 B)$ & $0.96(4)$ & $\mathrm{O}(65 \mathrm{~W})-\mathrm{H}(65 \mathrm{~A})$ & $0.957(11)$ \\
\hline $\mathrm{O}(65 \mathrm{~W})-\mathrm{H}(65 \mathrm{~B})$ & $0.958(11)$ & $\mathrm{O}(66 \mathrm{~W})-\mathrm{H}(66 \mathrm{~A})$ & $0.964(11)$ \\
\hline $\mathrm{O}(66 \mathrm{~W})-\mathrm{H}(66 \mathrm{~B})$ & $0.99(8)$ & $\mathrm{O}(67 \mathrm{~W})-\mathrm{H}(67 \mathrm{~B})$ & $0.970(11)$ \\
\hline $\mathrm{O}(67 \mathrm{~W})-\mathrm{H}(67 \mathrm{~A})$ & $0.97(2)$ & $\mathrm{O}(68 \mathrm{~W})-\mathrm{H}(68 \mathrm{~A})$ & $0.957(11)$ \\
\hline $\mathrm{O}(68 \mathrm{~W})-\mathrm{H}(68 \mathrm{~B})$ & $0.959(11)$ & $\mathrm{O}(69 \mathrm{~W})-\mathrm{O}(69 \mathrm{~V})$ & $0.67(2)$ \\
\hline $\mathrm{O}(69 \mathrm{~W})-\mathrm{H}(69 \mathrm{C})$ & $0.88(8)$ & $\mathrm{O}(69 \mathrm{~W})-\mathrm{H}(69 \mathrm{~A})$ & $0.94(4)$ \\
\hline
\end{tabular}




$\begin{array}{llll}\mathrm{O}(69 \mathrm{~W})-\mathrm{H}(69 \mathrm{~B}) & 0.957(11) & \mathrm{O}(69 \mathrm{~V})-\mathrm{H}(69 \mathrm{~A}) & 1.01(3) \\ \mathrm{O}(69 \mathrm{~V})-\mathrm{H}(69 \mathrm{C}) & 1.04(8) & \mathrm{H}(69 \mathrm{~B})-\mathrm{H}(69 \mathrm{C}) & 1.06(7) \\ \mathrm{H}(69 \mathrm{C})-\mathrm{H}(69 \mathrm{~B}) & 1.06(7) & \mathrm{O}(70 \mathrm{~W})-\mathrm{H}(70 \mathrm{~A}) & 0.961(11) \\ \mathrm{O}(70 \mathrm{~W})-\mathrm{H}(70 \mathrm{~B}) & 0.961(11) & \mathrm{O}(71 \mathrm{~W})-\mathrm{H}(71 \mathrm{~A}) & 0.951(11) \\ \mathrm{O}(71 \mathrm{~W})-\mathrm{H}(71 \mathrm{~A}) \# 12 & 0.951(11) & \mathrm{O}(71 \mathrm{~W})-\mathrm{H}(71 \mathrm{~B}) & 0.973(11) \\ \mathrm{O}(71 \mathrm{~W})-\mathrm{H}(71 \mathrm{~B}) \# 12 & 0.973(11) & \mathrm{O}(72 \mathrm{~W})-\mathrm{H}(72 \mathrm{~A}) & 0.95(5) \\ \mathrm{O}(72 \mathrm{~W})-\mathrm{H}(72 \mathrm{~B}) & 0.960(11) & \mathrm{O}(73 \mathrm{~W})-\mathrm{H}(73 \mathrm{~A}) & 0.958(11) \\ \mathrm{O}(73 \mathrm{~W})-\mathrm{H}(73 \mathrm{~B}) & 0.959(11) & \mathrm{O}(74 \mathrm{~W})-\mathrm{H}(74 \mathrm{~B}) & 0.958(11) \\ \mathrm{O}(74 \mathrm{~W})-\mathrm{H}(74 \mathrm{C}) & 0.959(11) & \mathrm{O}(74 \mathrm{~W})-\mathrm{H}(74 \mathrm{~A}) & 0.959(11) \\ \mathrm{O}(75 \mathrm{~W})-\mathrm{H}(75 \mathrm{~A}) & 0.955(11) & \mathrm{O}(75 \mathrm{~W})-\mathrm{H}(75 \mathrm{~B}) & 0.960(11) \\ \mathrm{O}(76 \mathrm{~W})-\mathrm{H}(76 \mathrm{~B}) & 0.969(11) & \mathrm{O}(76 \mathrm{~W})-\mathrm{H}(76 \mathrm{~A}) & 0.99(3) \\ \mathrm{O}(77 \mathrm{~W})-\mathrm{H}(77 \mathrm{~A}) & 0.957(11) & \mathrm{O}(77 \mathrm{~W})-\mathrm{H}(77 \mathrm{~B}) & 0.957(11)\end{array}$

Symmetry transformations used to generate equivalent atoms:

$\# 1-x+2,-y+3,-z-1 \quad \# 2-x+2,-y+3,-z \quad \# 3-x+3,-y+3,-z-1$

$\# 4 x, y+1, z \quad \# 5-x+1,-y+4,-z-1 \quad \# 6-x+2,-y+2,-z$

\#7-x+2,-y+4,-z-1 \#8x,y-1,z \#9-x+3,-y+2,-z

$\# 10 x+1, y, z \quad \# 11 x-1, y, z \quad \# 12-x+2,-y+1,-z+1$ 
Table S8: Bond angles [ $\left.{ }^{\circ}\right]$ for $\mathrm{Na} / \mathrm{K}\left\{\mathrm{U}_{24} \mathrm{Pp}_{12}\right\}$.

\begin{tabular}{|c|c|c|c|}
\hline atom-atom-atom & angle & atom-atom-atom & angle \\
\hline$O(5)-U(1)-O(3)$ & $178.5(5)$ & $O(5)-U(1)-O(1)$ & $89.9(4)$ \\
\hline $\mathrm{O}(3)-\mathrm{U}(1)-\mathrm{O}(1)$ & $90.5(4)$ & $O(5)-U(1)-O(4)$ & $87.3(4)$ \\
\hline$O(3)-U(1)-O(4)$ & $94.2(4)$ & $O(1)-U(1)-O(4)$ & $72.1(3)$ \\
\hline$O(5)-U(1)-O(2)$ & $91.8(4)$ & $O(3)-U(1)-O(2)$ & $89.4(4)$ \\
\hline$O(1)-U(1)-O(2)$ & $107.6(3)$ & $O(4)-U(1)-O(2)$ & $35.9(3)$ \\
\hline$O(5)-U(1)-O(6)$ & $91.0(4)$ & $O(3)-U(1)-O(6)$ & $88.5(4)$ \\
\hline$O(1)-U(1)-O(6)$ & $178.8(4)$ & $O(4)-U(1)-O(6)$ & $107.4(3)$ \\
\hline$O(2)-U(1)-O(6)$ & $71.7(3)$ & $O(5)-U(1)-O(8)$ & $94.1(4)$ \\
\hline$O(3)-U(1)-O(8)$ & $85.4(4)$ & $O(1)-U(1)-O(8)$ & $35.7(3)$ \\
\hline $\mathrm{O}(4)-\mathrm{U}(1)-\mathrm{O}(8)$ & $107.6(3)$ & $O(2)-U(1)-O(8)$ & $142.7(4)$ \\
\hline$O(6)-U(1)-O(8)$ & $144.8(3)$ & $O(5)-U(1)-O(7) \# 1$ & $84.2(4)$ \\
\hline$O(3)-U(1)-O(7) \# 1$ & $94.4(4)$ & $O(1)-U(1)-O(7) \# 1$ & $105.8(3)$ \\
\hline$O(4)-U(1)-O(7) \# 1$ & $171.3(3)$ & $O(2)-U(1)-O(7) \# 1$ & $146.3(4)$ \\
\hline$O(6)-U(1)-O(7) \# 1$ & 74.9(3) & $O(8)-U(1)-O(7) \# 1$ & $71.1(3)$ \\
\hline $\mathrm{O}(5)-\mathrm{U}(1)-\mathrm{Na}(1)$ & $158.3(4)$ & $\mathrm{O}(3)-\mathrm{U}(1)-\mathrm{Na}(1)$ & $22.2(3)$ \\
\hline $\mathrm{O}(1)-\mathrm{U}(1)-\mathrm{Na}(1)$ & $107.8(3)$ & $\mathrm{O}(4)-\mathrm{U}(1)-\mathrm{Na}(1)$ & $86.5(3)$ \\
\hline $\mathrm{O}(2)-\mathrm{U}(1)-\mathrm{Na}(1)$ & $71.2(3)$ & $\mathrm{O}(6)-\mathrm{U}(1)-\mathrm{Na}(1)$ & 71.1(3) \\
\hline $\mathrm{O}(8)-\mathrm{U}(1)-\mathrm{Na}(1)$ & $107.6(3)$ & $\mathrm{O}(7) \# 1-\mathrm{U}(1)-\mathrm{Na}(1)$ & $102.2(3)$ \\
\hline$O(12)-U(2)-O(14)$ & $177.7(5)$ & $\mathrm{O}(12)-\mathrm{U}(2)-\mathrm{O}(13)$ & $90.7(4)$ \\
\hline$O(14)-U(2)-O(13)$ & $90.5(4)$ & $\mathrm{O}(12)-\mathrm{U}(2)-\mathrm{O}(10)$ & $93.2(4)$ \\
\hline$O(14)-U(2)-O(10)$ & $89.0(4)$ & $O(13)-U(2)-O(10)$ & $72.7(3)$ \\
\hline$O(12)-U(2)-O(9)$ & $88.2(4)$ & $O(14)-U(2)-O(9)$ & $90.6(4)$ \\
\hline $\mathrm{O}(13)-\mathrm{U}(2)-\mathrm{O}(9)$ & $178.3(3)$ & $O(10)-U(2)-O(9)$ & $106.0(3)$ \\
\hline$O(12)-U(2)-O(15)$ & $86.2(4)$ & $O(14)-U(2)-O(15)$ & $93.6(4)$ \\
\hline$O(13)-U(2)-O(15)$ & $36.3(3)$ & $O(10)-U(2)-O(15)$ & $108.9(4)$ \\
\hline$O(9)-U(2)-O(15)$ & $144.8(4)$ & $O(12)-U(2)-O(42)$ & $89.0(3)$ \\
\hline$O(14)-U(2)-O(42)$ & $92.5(4)$ & $O(13)-U(2)-O(42)$ & $108.5(3)$ \\
\hline$O(10)-U(2)-O(42)$ & $36.0(3)$ & $O(9)-U(2)-O(42)$ & $70.2(3)$ \\
\hline$O(15)-U(2)-O(42)$ & $144.2(4)$ & $\mathrm{O}(12)-\mathrm{U}(2)-\mathrm{O}(11)$ & $91.9(4)$ \\
\hline$O(14)-U(2)-O(11)$ & $85.8(4)$ & $\mathrm{O}(13)-\mathrm{U}(2)-\mathrm{O}(11)$ & 106.9(3) \\
\hline$O(10)-U(2)-O(11)$ & $174.8(4)$ & $O(9)-U(2)-O(11)$ & $74.5(3)$ \\
\hline$O(15)-U(2)-O(11)$ & 71.1(3) & $\mathrm{O}(42)-\mathrm{U}(2)-\mathrm{O}(11)$ & $144.6(4)$ \\
\hline$O(12)-U(2)-K(1)$ & $45.9(3)$ & $O(14)-U(2)-K(1)$ & $132.2(4)$ \\
\hline$O(13)-U(2)-K(1)$ & $87.0(3)$ & $O(10)-U(2)-K(1)$ & $134.7(3)$ \\
\hline$O(9)-U(2)-K(1)$ & $93.2(3)$ & $\mathrm{O}(15)-\mathrm{U}(2)-\mathrm{K}(1)$ & $58.9(3)$ \\
\hline$O(42)-U(2)-K(1)$ & $133.3(3)$ & $O(11)-U(2)-K(1)$ & $50.0(3)$ \\
\hline $\mathrm{O}(12)-\mathrm{U}(2)-\mathrm{Na}(11)$ & $24.6(3)$ & $\mathrm{O}(14)-\mathrm{U}(2)-\mathrm{Na}(11)$ & $155.7(4)$ \\
\hline $\mathrm{O}(13)-\mathrm{U}(2)-\mathrm{Na}(11)$ & $109.0(3)$ & $\mathrm{O}(10)-\mathrm{U}(2)-\mathrm{Na}(11)$ & $83.4(3)$ \\
\hline $\mathrm{O}(9)-\mathrm{U}(2)-\mathrm{Na}(11)$ & $69.6(3)$ & $\mathrm{O}(15)-\mathrm{U}(2)-\mathrm{Na}(11)$ & $110.7(3)$ \\
\hline
\end{tabular}




\begin{tabular}{|c|c|c|c|}
\hline $\mathrm{O}(42)-\mathrm{U}(2)-\mathrm{Na}(11)$ & $68.0(3)$ & $\mathrm{O}(11)-\mathrm{U}(2)-\mathrm{Na}(11)$ & 101.6(3) \\
\hline $\mathrm{K}(1)-\mathrm{U}(2)-\mathrm{Na}(11)$ & $65.3(3)$ & $\mathrm{O}(12)-\mathrm{U}(2)-\mathrm{Na}(2)$ & $163.8(4)$ \\
\hline $\mathrm{O}(14)-\mathrm{U}(2)-\mathrm{Na}(2)$ & $18.3(4)$ & $\mathrm{O}(13)-\mathrm{U}(2)-\mathrm{Na}(2)$ & $76.0(3)$ \\
\hline $\mathrm{O}(10)-\mathrm{U}(2)-\mathrm{Na}(2)$ & $74.1(3)$ & $\mathrm{O}(9)-\mathrm{U}(2)-\mathrm{Na}(2)$ & $104.8(3)$ \\
\hline $\mathrm{O}(15)-\mathrm{U}(2)-\mathrm{Na}(2)$ & $88.4(3)$ & $\mathrm{O}(42)-\mathrm{U}(2)-\mathrm{Na}(2)$ & $86.5(3)$ \\
\hline $\mathrm{O}(11)-\mathrm{U}(2)-\mathrm{Na}(2)$ & $100.7(3)$ & $\mathrm{K}(1)-\mathrm{U}(2)-\mathrm{Na}(2)$ & $140.1(3)$ \\
\hline $\mathrm{Na}(11)-\mathrm{U}(2)-\mathrm{Na}(2)$ & $154.4(3)$ & $O(43)-U(3)-O(22)$ & $178.8(5)$ \\
\hline$O(43)-U(3)-O(16)$ & $86.6(4)$ & $O(22)-U(3)-O(16)$ & $94.5(4)$ \\
\hline $\mathrm{O}(43)-\mathrm{U}(3)-\mathrm{O}(18)$ & $87.9(4)$ & $\mathrm{O}(22)-\mathrm{U}(3)-\mathrm{O}(18)$ & $92.3(4)$ \\
\hline $\mathrm{O}(16)-\mathrm{U}(3)-\mathrm{O}(18)$ & $69.0(3)$ & $O(43)-U(3)-O(19)$ & $90.0(4)$ \\
\hline $\mathrm{O}(22)-\mathrm{U}(3)-\mathrm{O}(19)$ & $91.1(4)$ & $O(16)-U(3)-O(19)$ & $36.4(3)$ \\
\hline $\mathrm{O}(18)-\mathrm{U}(3)-\mathrm{O}(19)$ & $105.3(3)$ & $\mathrm{O}(43)-\mathrm{U}(3)-\mathrm{O}(21)$ & $86.9(4)$ \\
\hline$O(22)-U(3)-O(21)$ & $91.9(4)$ & $O(16)-U(3)-O(21)$ & $172.1(4)$ \\
\hline $\mathrm{O}(18)-\mathrm{U}(3)-\mathrm{O}(21)$ & $106.2(3)$ & $O(19)-U(3)-O(21)$ & $148.2(3)$ \\
\hline $\mathrm{O}(43)-U(3)-O(20) \# 1$ & $91.5(4)$ & $\mathrm{O}(22)-\mathrm{U}(3)-\mathrm{O}(20) \# 1$ & $88.3(4)$ \\
\hline $\mathrm{O}(16)-\mathrm{U}(3)-\mathrm{O}(20) \# 1$ & $111.4(3)$ & $\mathrm{O}(18)-\mathrm{U}(3)-\mathrm{O}(20) \# 1$ & $179.3(4)$ \\
\hline $\mathrm{O}(19)-U(3)-\mathrm{O}(20) \# 1$ & $75.1(3)$ & $\mathrm{O}(21)-\mathrm{U}(3)-\mathrm{O}(20) \# 1$ & $73.4(3)$ \\
\hline$O(43)-U(3)-O(17)$ & $91.3(4)$ & $\mathrm{O}(22)-\mathrm{U}(3)-\mathrm{O}(17)$ & $88.2(4)$ \\
\hline $\mathrm{O}(16)-\mathrm{U}(3)-\mathrm{O}(17)$ & $105.1(3)$ & $\mathrm{O}(18)-\mathrm{U}(3)-\mathrm{O}(17)$ & $36.2(2)$ \\
\hline $\mathrm{O}(19)-\mathrm{U}(3)-\mathrm{O}(17)$ & $141.4(3)$ & $\mathrm{O}(21)-\mathrm{U}(3)-\mathrm{O}(17)$ & $70.4(3)$ \\
\hline $\mathrm{O}(20) \# 1-\mathrm{U}(3)-\mathrm{O}(17)$ & $143.4(3)$ & $\mathrm{O}(43)-\mathrm{U}(3)-\mathrm{Na}(19)$ & $69.5(5)$ \\
\hline $\mathrm{O}(22)-\mathrm{U}(3)-\mathrm{Na}(19)$ & $109.5(5)$ & $\mathrm{O}(16)-\mathrm{U}(3)-\mathrm{Na}(19)$ & $129.8(4)$ \\
\hline $\mathrm{O}(18)-\mathrm{U}(3)-\mathrm{Na}(19)$ & $66.8(4)$ & $\mathrm{O}(19)-\mathrm{U}(3)-\mathrm{Na}(19)$ & $157.8(5)$ \\
\hline $\mathrm{O}(21)-\mathrm{U}(3)-\mathrm{Na}(19)$ & $43.0(4)$ & $\mathrm{O}(20) \# 1-\mathrm{U}(3)-\mathrm{Na}(19)$ & $112.6(4)$ \\
\hline $\mathrm{O}(17)-\mathrm{U}(3)-\mathrm{Na}(19)$ & $36.5(4)$ & $\mathrm{O}(43)-\mathrm{U}(3)-\mathrm{Na}(3)$ & $156.9(4)$ \\
\hline $\mathrm{O}(22)-\mathrm{U}(3)-\mathrm{Na}(3)$ & $24.0(3)$ & $\mathrm{O}(16)-\mathrm{U}(3)-\mathrm{Na}(3)$ & $73.7(3)$ \\
\hline $\mathrm{O}(18)-\mathrm{U}(3)-\mathrm{Na}(3)$ & $73.9(3)$ & $\mathrm{O}(19)-\mathrm{U}(3)-\mathrm{Na}(3)$ & $81.5(3)$ \\
\hline $\mathrm{O}(21)-\mathrm{U}(3)-\mathrm{Na}(3)$ & $111.5(3)$ & $\mathrm{O}(20) \# 1-\mathrm{U}(3)-\mathrm{Na}(3)$ & $106.8(3)$ \\
\hline $\mathrm{O}(17)-\mathrm{U}(3)-\mathrm{Na}(3)$ & $82.5(3)$ & $\mathrm{Na}(19)-\mathrm{U}(3)-\mathrm{Na}(3)$ & $114.1(4)$ \\
\hline $\mathrm{O}(43)-\mathrm{U}(3)-\mathrm{Na}(1) \# 1$ & $18.7(3)$ & $\mathrm{O}(22)-\mathrm{U}(3)-\mathrm{Na}(1) \# 1$ & $162.4(4)$ \\
\hline $\mathrm{O}(16)-\mathrm{U}(3)-\mathrm{Na}(1) \# 1$ & $71.0(3)$ & $\mathrm{O}(18)-\mathrm{U}(3)-\mathrm{Na}(1) \# 1$ & $91.7(3)$ \\
\hline $\mathrm{O}(19)-\mathrm{U}(3)-\mathrm{Na}(1) \# 1$ & $71.3(3)$ & $\mathrm{O}(21)-\mathrm{U}(3)-\mathrm{Na}(1) \# 1$ & 103.3(3) \\
\hline $\mathrm{O}(20) \# 1-\mathrm{U}(3)-\mathrm{Na}(1) \# 1$ & $87.8(3)$ & $\mathrm{O}(17)-\mathrm{U}(3)-\mathrm{Na}(1) \# 1$ & $105.0(3)$ \\
\hline $\mathrm{Na}(19)-\mathrm{U}(3)-\mathrm{Na}(1) \# 1$ & $87.8(4)$ & $\mathrm{Na}(3)-\mathrm{U}(3)-\mathrm{Na}(1) \# 1$ & $144.7(3)$ \\
\hline $\mathrm{O}(25)-\mathrm{U}(4)-\mathrm{O}(45)$ & $179.0(5)$ & $\mathrm{O}(25)-\mathrm{U}(4)-\mathrm{O}(18)$ & $87.2(4)$ \\
\hline $\mathrm{O}(45)-\mathrm{U}(4)-\mathrm{O}(18)$ & $93.8(4)$ & $\mathrm{O}(25)-\mathrm{U}(4)-\mathrm{O}(24)$ & $86.1(4)$ \\
\hline $\mathrm{O}(45)-\mathrm{U}(4)-\mathrm{O}(24)$ & $94.1(4)$ & $\mathrm{O}(18)-\mathrm{U}(4)-\mathrm{O}(24)$ & $68.0(3)$ \\
\hline $\mathrm{O}(25)-\mathrm{U}(4)-\mathrm{O}(17)$ & $89.6(4)$ & $\mathrm{O}(45)-\mathrm{U}(4)-\mathrm{O}(17)$ & $91.3(4)$ \\
\hline $\mathrm{O}(18)-\mathrm{U}(4)-\mathrm{O}(17)$ & $36.4(2)$ & $\mathrm{O}(24)-\mathrm{U}(4)-\mathrm{O}(17)$ & $104.4(3)$ \\
\hline $\mathrm{O}(25)-\mathrm{U}(4)-\mathrm{O}(85)$ & $88.5(4)$ & $\mathrm{O}(45)-\mathrm{U}(4)-\mathrm{O}(85)$ & $91.0(4)$ \\
\hline $\mathrm{O}(18)-\mathrm{U}(4)-\mathrm{O}(85)$ & $104.6(3)$ & $\mathrm{O}(24)-\mathrm{U}(4)-\mathrm{O}(85)$ & $36.6(3)$ \\
\hline $\mathrm{O}(17)-U(4)-O(85)$ & $141.0(3)$ & $\mathrm{O}(25)-\mathrm{U}(4)-\mathrm{O}(23)$ & $87.1(4)$ \\
\hline $\mathrm{O}(45)-\mathrm{U}(4)-\mathrm{O}(23)$ & $91.9(4)$ & $\mathrm{O}(18)-\mathrm{U}(4)-\mathrm{O}(23)$ & $172.3(4)$ \\
\hline $\mathrm{O}(24)-\mathrm{U}(4)-\mathrm{O}(23)$ & 106.6(3) & $\mathrm{O}(17)-\mathrm{U}(4)-\mathrm{O}(23)$ & $148.6(3)$ \\
\hline
\end{tabular}




\begin{tabular}{|c|c|c|c|}
\hline $\mathrm{O}(85)-\mathrm{U}(4)-\mathrm{O}(23)$ & $70.2(3)$ & $\mathrm{O}(25)-\mathrm{U}(4)-\mathrm{O}(44)$ & $90.2(4)$ \\
\hline $\mathrm{O}(45)-\mathrm{U}(4)-\mathrm{O}(44)$ & $89.5(4)$ & $\mathrm{O}(18)-\mathrm{U}(4)-\mathrm{O}(44)$ & $112.2(3)$ \\
\hline $\mathrm{O}(24)-\mathrm{U}(4)-\mathrm{O}(44)$ & $176.4(4)$ & $\mathrm{O}(17)-\mathrm{U}(4)-\mathrm{O}(44)$ & $75.8(3)$ \\
\hline $\mathrm{O}(85)-\mathrm{U}(4)-\mathrm{O}(44)$ & $143.2(3)$ & $\mathrm{O}(23)-\mathrm{U}(4)-\mathrm{O}(44)$ & $73.0(3)$ \\
\hline $\mathrm{O}(25)-\mathrm{U}(4)-\mathrm{K}(1)$ & $44.9(3)$ & $\mathrm{O}(45)-\mathrm{U}(4)-\mathrm{K}(1)$ & $134.3(4)$ \\
\hline $\mathrm{O}(18)-\mathrm{U}(4)-\mathrm{K}(1)$ & $115.5(3)$ & $\mathrm{O}(24)-\mathrm{U}(4)-\mathrm{K}(1)$ & $68.6(3)$ \\
\hline $\mathrm{O}(17)-\mathrm{U}(4)-\mathrm{K}(1)$ & $133.3(3)$ & $\mathrm{O}(85)-\mathrm{U}(4)-\mathrm{K}(1)$ & $49.5(3)$ \\
\hline $\mathrm{O}(23)-U(4)-K(1)$ & $56.8(3)$ & $\mathrm{O}(44)-\mathrm{U}(4)-\mathrm{K}(1)$ & $108.6(3)$ \\
\hline $\mathrm{O}(25)-\mathrm{U}(4)-\mathrm{Na}(3)$ & $156.7(4)$ & $\mathrm{O}(45)-\mathrm{U}(4)-\mathrm{Na}(3)$ & $24.1(3)$ \\
\hline $\mathrm{O}(18)-\mathrm{U}(4)-\mathrm{Na}(3)$ & $72.9(3)$ & $\mathrm{O}(24)-\mathrm{U}(4)-\mathrm{Na}(3)$ & $75.4(3)$ \\
\hline $\mathrm{O}(17)-\mathrm{U}(4)-\mathrm{Na}(3)$ & $81.6(3)$ & $\mathrm{O}(85)-\mathrm{U}(4)-\mathrm{Na}(3)$ & $85.0(3)$ \\
\hline $\mathrm{O}(23)-\mathrm{U}(4)-\mathrm{Na}(3)$ & $111.5(3)$ & $\mathrm{O}(44)-\mathrm{U}(4)-\mathrm{Na}(3)$ & $108.2(3)$ \\
\hline $\mathrm{K}(1)-\mathrm{U}(4)-\mathrm{Na}(3)$ & $134.5(3)$ & $\mathrm{O}(25)-\mathrm{U}(4)-\mathrm{Na}(11)$ & $19.8(3)$ \\
\hline $\mathrm{O}(45)-\mathrm{U}(4)-\mathrm{Na}(11)$ & $161.2(4)$ & $\mathrm{O}(18)-\mathrm{U}(4)-\mathrm{Na}(11)$ & $70.6(3)$ \\
\hline $\mathrm{O}(24)-\mathrm{U}(4)-\mathrm{Na}(11)$ & $89.9(3)$ & $\mathrm{O}(17)-\mathrm{U}(4)-\mathrm{Na}(11)$ & $69.9(3)$ \\
\hline $\mathrm{O}(85)-\mathrm{U}(4)-\mathrm{Na}(11)$ & $102.9(3)$ & $\mathrm{O}(23)-\mathrm{U}(4)-\mathrm{Na}(11)$ & 104.6(3) \\
\hline $\mathrm{O}(44)-\mathrm{U}(4)-\mathrm{Na}(11)$ & $86.8(3)$ & $\mathrm{K}(1)-\mathrm{U}(4)-\mathrm{Na}(11)$ & $64.1(3)$ \\
\hline $\mathrm{Na}(3)-\mathrm{U}(4)-\mathrm{Na}(11)$ & $143.5(3)$ & $O(29)-U(5)-O(46)$ & $178.1(5)$ \\
\hline$O(29)-U(5)-O(16)$ & $86.3(4)$ & $O(46)-U(5)-O(16)$ & $93.8(4)$ \\
\hline $\mathrm{O}(29)-\mathrm{U}(5)-\mathrm{O}(26)$ & $87.6(4)$ & $\mathrm{O}(46)-\mathrm{U}(5)-\mathrm{O}(26)$ & $94.3(4)$ \\
\hline $\mathrm{O}(16)-\mathrm{U}(5)-\mathrm{O}(26)$ & $68.7(3)$ & $\mathrm{O}(29)-\mathrm{U}(5)-\mathrm{O}(28) \# 1$ & $89.4(4)$ \\
\hline $\mathrm{O}(46)-\mathrm{U}(5)-\mathrm{O}(28) \# 1$ & $90.4(4)$ & $\mathrm{O}(16)-\mathrm{U}(5)-\mathrm{O}(28) \# 1$ & $175.7(4)$ \\
\hline $\mathrm{O}(26)-\mathrm{U}(5)-\mathrm{O}(28) \# 1$ & $111.3(3)$ & $O(29)-U(5)-O(19)$ & $87.5(4)$ \\
\hline $\mathrm{O}(46)-\mathrm{U}(5)-\mathrm{O}(19)$ & $91.5(4)$ & $O(16)-U(5)-O(19)$ & $36.1(3)$ \\
\hline$O(26)-U(5)-O(19)$ & $104.8(3)$ & $\mathrm{O}(28) \# 1-\mathrm{U}(5)-\mathrm{O}(19)$ & $143.6(4)$ \\
\hline$O(29)-U(5)-O(30)$ & $90.7(4)$ & $O(46)-U(5)-O(30)$ & $91.1(4)$ \\
\hline $\mathrm{O}(16)-\mathrm{U}(5)-\mathrm{O}(30)$ & $104.9(3)$ & $\mathrm{O}(26)-\mathrm{U}(5)-\mathrm{O}(30)$ & $36.2(3)$ \\
\hline $\mathrm{O}(28) \# 1-\mathrm{U}(5)-\mathrm{O}(30)$ & $75.3(3)$ & $O(19)-U(5)-O(30)$ & $141.0(4)$ \\
\hline $\mathrm{O}(29)-U(5)-O(27) \# 1$ & $88.3(4)$ & $\mathrm{O}(46)-\mathrm{U}(5)-\mathrm{O}(27) \# 1$ & $89.8(4)$ \\
\hline $\mathrm{O}(16)-\mathrm{U}(5)-\mathrm{O}(27) \# 1$ & 107.7(3) & $\mathrm{O}(26)-\mathrm{U}(5)-\mathrm{O}(27) \# 1$ & $174.7(4)$ \\
\hline $\mathrm{O}(28) \# 1-\mathrm{U}(5)-\mathrm{O}(27) \# 1$ & $72.0(3)$ & $\mathrm{O}(19)-\mathrm{U}(5)-\mathrm{O}(27) \# 1$ & $71.6(3)$ \\
\hline $\mathrm{O}(30)-U(5)-\mathrm{O}(27) \# 1$ & $147.3(3)$ & $O(29)-U(5)-K(2) \# 1$ & $43.5(3)$ \\
\hline$O(46)-U(5)-K(2) \# 1$ & $134.9(4)$ & $O(16)-U(5)-K(2) \# 1$ & $68.5(3)$ \\
\hline $\mathrm{O}(26)-\mathrm{U}(5)-\mathrm{K}(2) \# 1$ & $114.9(4)$ & $\mathrm{O}(28) \# 1-\mathrm{U}(5)-\mathrm{K}(2) \# 1$ & $108.1(3)$ \\
\hline $\mathrm{O}(19)-\mathrm{U}(5)-\mathrm{K}(2) \# 1$ & $49.5(3)$ & $O(30)-U(5)-K(2) \# 1$ & $132.8(3)$ \\
\hline $\mathrm{O}(27) \# 1-\mathrm{U}(5)-\mathrm{K}(2) \# 1$ & $59.8(3)$ & $\mathrm{O}(29)-\mathrm{U}(5)-\mathrm{Na}(3)$ & $158.7(4)$ \\
\hline $\mathrm{O}(46)-\mathrm{U}(5)-\mathrm{Na}(3)$ & $22.2(4)$ & $\mathrm{O}(16)-\mathrm{U}(5)-\mathrm{Na}(3)$ & $74.2(3)$ \\
\hline $\mathrm{O}(26)-\mathrm{U}(5)-\mathrm{Na}(3)$ & $77.6(3)$ & $\mathrm{O}(28) \# 1-\mathrm{U}(5)-\mathrm{Na}(3)$ & $110.1(3)$ \\
\hline $\mathrm{O}(19)-\mathrm{U}(5)-\mathrm{Na}(3)$ & $81.7(3)$ & $\mathrm{O}(30)-\mathrm{U}(5)-\mathrm{Na}(3)$ & $86.3(3)$ \\
\hline $\mathrm{O}(27) \# 1-\mathrm{U}(5)-\mathrm{Na}(3)$ & $105.4(3)$ & $\mathrm{K}(2) \# 1-\mathrm{U}(5)-\mathrm{Na}(3)$ & $131.0(3)$ \\
\hline $\mathrm{O}(47)-\mathrm{U}(6)-\mathrm{O}(48)$ & $176.1(5)$ & $\mathrm{O}(47)-\mathrm{U}(6)-\mathrm{O}(33)$ & $91.7(4)$ \\
\hline $\mathrm{O}(48)-\mathrm{U}(6)-\mathrm{O}(33)$ & $91.2(4)$ & $\mathrm{O}(47)-\mathrm{U}(6)-\mathrm{O}(1)$ & $94.5(4)$ \\
\hline$O(48)-U(6)-O(1)$ & $88.8(4)$ & $O(33)-U(6)-O(1)$ & $70.1(3)$ \\
\hline $\mathrm{O}(47)-U(6)-O(8)$ & $93.8(4)$ & $\mathrm{O}(48)-U(6)-O(8)$ & $87.8(4)$ \\
\hline
\end{tabular}




\begin{tabular}{|c|c|c|c|}
\hline $\mathrm{O}(33)-\mathrm{U}(6)-\mathrm{O}(8)$ & $106.0(3)$ & $\mathrm{O}(1)-\mathrm{U}(6)-\mathrm{O}(8)$ & $35.9(3)$ \\
\hline$O(47)-U(6)-O(31)$ & $83.1(4)$ & $\mathrm{O}(48)-\mathrm{U}(6)-\mathrm{O}(31)$ & $94.0(4)$ \\
\hline$O(33)-U(6)-O(31)$ & $174.6(4)$ & $O(1)-U(6)-O(31)$ & $108.5(4)$ \\
\hline $\mathrm{O}(8)-U(6)-O(31)$ & $72.7(3)$ & $\mathrm{O}(47)-\mathrm{U}(6)-\mathrm{O}(49)$ & $88.4(4)$ \\
\hline$O(48)-U(6)-O(49)$ & $92.5(4)$ & $\mathrm{O}(33)-\mathrm{U}(6)-\mathrm{O}(49)$ & $35.8(3)$ \\
\hline $\mathrm{O}(1)-\mathrm{U}(6)-\mathrm{O}(49)$ & $105.9(3)$ & $\mathrm{O}(8)-\mathrm{U}(6)-\mathrm{O}(49)$ & $141.8(4)$ \\
\hline$O(31)-U(6)-O(49)$ & $145.1(4)$ & $\mathrm{O}(47)-\mathrm{U}(6)-\mathrm{O}(32)$ & $88.6(4)$ \\
\hline$O(48)-U(6)-O(32)$ & $88.1(4)$ & $\mathrm{O}(33)-\mathrm{U}(6)-\mathrm{O}(32)$ & $108.7(3)$ \\
\hline$O(1)-U(6)-O(32)$ & $176.7(4)$ & $O(8)-U(6)-O(32)$ & $145.2(4)$ \\
\hline$O(31)-U(6)-O(32)$ & $73.1(3)$ & $\mathrm{O}(49)-\mathrm{U}(6)-\mathrm{O}(32)$ & $72.9(3)$ \\
\hline $\mathrm{O}(47)-\mathrm{U}(6)-\mathrm{K}(3)$ & $50.2(4)$ & $\mathrm{O}(48)-\mathrm{U}(6)-\mathrm{K}(3)$ & $128.0(4)$ \\
\hline$O(33)-U(6)-K(3)$ & $80.8(3)$ & $O(1)-U(6)-K(3)$ & $133.6(4)$ \\
\hline$O(8)-U(6)-K(3)$ & $144.0(4)$ & $\mathrm{O}(31)-U(6)-K(3)$ & $97.2(3)$ \\
\hline $\mathrm{O}(49)-U(6)-K(3)$ & $53.2(3)$ & $\mathrm{O}(32)-U(6)-K(3)$ & $48.2(3)$ \\
\hline $\mathrm{O}(38)-\mathrm{U}(7)-\mathrm{O}(36)$ & $179.3(5)$ & $\mathrm{O}(38)-\mathrm{U}(7)-\mathrm{O}(26)$ & $94.4(4)$ \\
\hline$O(36)-U(7)-O(26)$ & $86.1(4)$ & $\mathrm{O}(38)-\mathrm{U}(7)-\mathrm{O}(24)$ & $91.9(4)$ \\
\hline$O(36)-U(7)-O(24)$ & $88.7(4)$ & $\mathrm{O}(26)-\mathrm{U}(7)-\mathrm{O}(24)$ & $68.6(3)$ \\
\hline$O(38)-U(7)-O(30)$ & $92.0(4)$ & $\mathrm{O}(36)-\mathrm{U}(7)-\mathrm{O}(30)$ & $88.1(4)$ \\
\hline$O(26)-U(7)-O(30)$ & $36.6(3)$ & $\mathrm{O}(24)-\mathrm{U}(7)-\mathrm{O}(30)$ & $105.2(3)$ \\
\hline $\mathrm{O}(38)-\mathrm{U}(7)-\mathrm{O}(37)$ & $89.0(4)$ & $\mathrm{O}(36)-\mathrm{U}(7)-\mathrm{O}(37)$ & $90.4(4)$ \\
\hline$O(26)-U(7)-O(37)$ & $108.0(3)$ & $\mathrm{O}(24)-\mathrm{U}(7)-\mathrm{O}(37)$ & $176.5(4)$ \\
\hline$O(30)-U(7)-O(37)$ & $71.5(3)$ & $\mathrm{O}(38)-\mathrm{U}(7)-\mathrm{O}(34)$ & $89.8(4)$ \\
\hline$O(36)-U(7)-O(34)$ & $89.7(4)$ & $\mathrm{O}(26)-\mathrm{U}(7)-\mathrm{O}(34)$ & $175.6(4)$ \\
\hline$O(24)-U(7)-O(34)$ & $110.3(3)$ & $\mathrm{O}(30)-\mathrm{U}(7)-\mathrm{O}(34)$ & $144.4(3)$ \\
\hline$O(37)-U(7)-O(34)$ & $73.0(3)$ & $\mathrm{O}(38)-\cup(7)-O(85)$ & $89.2(4)$ \\
\hline$O(36)-U(7)-O(85)$ & $91.1(4)$ & $\mathrm{O}(26)-\mathrm{U}(7)-\mathrm{O}(85)$ & $105.0(3)$ \\
\hline$O(24)-U(7)-O(85)$ & $36.4(3)$ & $\mathrm{O}(30)-\mathrm{U}(7)-\mathrm{O}(85)$ & $141.5(3)$ \\
\hline $\mathrm{O}(37)-\mathrm{U}(7)-\mathrm{O}(85)$ & $147.0(3)$ & $\mathrm{O}(34)-\mathrm{U}(7)-\mathrm{O}(85)$ & $74.1(3)$ \\
\hline $\mathrm{O}(38)-U(7)-K(3)$ & $135.1(4)$ & $\mathrm{O}(36)-U(7)-K(3)$ & $44.6(4)$ \\
\hline $\mathrm{O}(26)-U(7)-K(3)$ & $71.0(3)$ & $\mathrm{O}(24)-U(7)-K(3)$ & $118.9(4)$ \\
\hline $\mathrm{O}(30)-U(7)-K(3)$ & $50.9(3)$ & $\mathrm{O}(37)-U(7)-K(3)$ & $58.5(4)$ \\
\hline $\mathrm{O}(34)-U(7)-K(3)$ & $106.6(3)$ & $\mathrm{O}(85)-U(7)-K(3)$ & $135.1(4)$ \\
\hline $\mathrm{O}(38)-\mathrm{U}(7)-\mathrm{Na}(7)$ & $159.1(4)$ & $\mathrm{O}(36)-\mathrm{U}(7)-\mathrm{Na}(7)$ & $21.3(3)$ \\
\hline $\mathrm{O}(26)-\mathrm{U}(7)-\mathrm{Na}(7)$ & $90.7(3)$ & $\mathrm{O}(24)-\mathrm{U}(7)-\mathrm{Na}(7)$ & 71.1(3) \\
\hline $\mathrm{O}(30)-\mathrm{U}(7)-\mathrm{Na}(7)$ & $103.9(3)$ & $\mathrm{O}(37)-\mathrm{U}(7)-\mathrm{Na}(7)$ & $108.7(3)$ \\
\hline $\mathrm{O}(34)-\mathrm{U}(7)-\mathrm{Na}(7)$ & $85.0(3)$ & $\mathrm{O}(85)-\mathrm{U}(7)-\mathrm{Na}(7)$ & $69.9(3)$ \\
\hline $\mathrm{K}(3)-\mathrm{U}(7)-\mathrm{Na}(7)$ & $65.6(3)$ & $\mathrm{O}(38)-\mathrm{U}(7)-\mathrm{Na}(3)$ & $23.6(3)$ \\
\hline $\mathrm{O}(36)-\mathrm{U}(7)-\mathrm{Na}(3)$ & $157.1(4)$ & $\mathrm{O}(26)-\mathrm{U}(7)-\mathrm{Na}(3)$ & $74.3(3)$ \\
\hline $\mathrm{O}(24)-\mathrm{U}(7)-\mathrm{Na}(3)$ & $73.4(3)$ & $\mathrm{O}(30)-\mathrm{U}(7)-\mathrm{Na}(3)$ & $83.1(3)$ \\
\hline $\mathrm{O}(37)-\mathrm{U}(7)-\mathrm{Na}(3)$ & $106.6(3)$ & $\mathrm{O}(34)-\mathrm{U}(7)-\mathrm{Na}(3)$ & 109.7(3) \\
\hline $\mathrm{O}(85)-\mathrm{U}(7)-\mathrm{Na}(3)$ & $82.9(3)$ & $\mathrm{K}(3)-\mathrm{U}(7)-\mathrm{Na}(3)$ & $133.7(3)$ \\
\hline $\mathrm{Na}(7)-\mathrm{U}(7)-\mathrm{Na}(3)$ & $144.4(3)$ & $\mathrm{O}(40)-\mathrm{U}(8)-\mathrm{O}(41)$ & $176.0(5)$ \\
\hline$O(40)-U(8)-O(39)$ & $90.2(4)$ & $\mathrm{O}(41)-\mathrm{U}(8)-\mathrm{O}(39)$ & $93.2(4)$ \\
\hline$O(40)-U(8)-O(13)$ & $88.8(4)$ & $\mathrm{O}(41)-\mathrm{U}(8)-\mathrm{O}(13)$ & $94.4(4)$ \\
\hline
\end{tabular}




\begin{tabular}{|c|c|c|c|}
\hline $\mathrm{O}(39)-\mathrm{U}(8)-\mathrm{O}(13)$ & $69.3(3)$ & $\mathrm{O}(40)-\mathrm{U}(8)-\mathrm{O}(86)$ & $91.4(4)$ \\
\hline $\mathrm{O}(41)-\mathrm{U}(8)-\mathrm{O}(86)$ & $85.5(4)$ & $\mathrm{O}(39)-\mathrm{U}(8)-\mathrm{O}(86)$ & $109.8(4)$ \\
\hline $\mathrm{O}(13)-\mathrm{U}(8)-\mathrm{O}(86)$ & $179.2(4)$ & $\mathrm{O}(40)-\mathrm{U}(8)-\mathrm{O}(35)$ & $89.9(4)$ \\
\hline $\mathrm{O}(41)-\mathrm{U}(8)-\mathrm{O}(35)$ & $91.6(4)$ & $\mathrm{O}(39)-\mathrm{U}(8)-\mathrm{O}(35)$ & $36.1(3)$ \\
\hline $\mathrm{O}(13)-\mathrm{U}(8)-\mathrm{O}(35)$ & $105.4(3)$ & $\mathrm{O}(86)-\mathrm{U}(8)-\mathrm{O}(35)$ & $73.8(3)$ \\
\hline $\mathrm{O}(40)-\mathrm{U}(8)-\mathrm{O}(50)$ & $92.2(4)$ & $\mathrm{O}(41)-\mathrm{U}(8)-\mathrm{O}(50)$ & $84.4(4)$ \\
\hline $\mathrm{O}(39)-\mathrm{U}(8)-\mathrm{O}(50)$ & $176.6(4)$ & $\mathrm{O}(13)-\mathrm{U}(8)-\mathrm{O}(50)$ & $108.3(4)$ \\
\hline $\mathrm{O}(86)-\mathrm{U}(8)-\mathrm{O}(50)$ & $72.5(4)$ & $O(35)-U(8)-O(50)$ & $146.3(4)$ \\
\hline $\mathrm{O}(40)-\mathrm{U}(8)-\mathrm{O}(15)$ & $89.1(4)$ & $\mathrm{O}(41)-\mathrm{U}(8)-\mathrm{O}(15)$ & $92.0(4)$ \\
\hline $\mathrm{O}(39)-\mathrm{U}(8)-\mathrm{O}(15)$ & $105.2(3)$ & $\mathrm{O}(13)-\mathrm{U}(8)-\mathrm{O}(15)$ & $35.8(3)$ \\
\hline $\mathrm{O}(86)-\mathrm{U}(8)-\mathrm{O}(15)$ & $145.0(4)$ & $\mathrm{O}(35)-\mathrm{U}(8)-\mathrm{O}(15)$ & $141.2(3)$ \\
\hline $\mathrm{O}(50)-\mathrm{U}(8)-\mathrm{O}(15)$ & $72.5(3)$ & $\mathrm{O}(40)-\mathrm{U}(8)-\mathrm{K}(1)$ & $126.9(4)$ \\
\hline $\mathrm{O}(41)-\mathrm{U}(8)-\mathrm{K}(1)$ & $51.4(4)$ & $\mathrm{O}(39)-U(8)-K(1)$ & $132.6(3)$ \\
\hline $\mathrm{O}(13)-\mathrm{U}(8)-\mathrm{K}(1)$ & $81.8(3)$ & $\mathrm{O}(86)-\mathrm{U}(8)-\mathrm{K}(1)$ & $98.8(4)$ \\
\hline $\mathrm{O}(35)-\mathrm{U}(8)-\mathrm{K}(1)$ & $143.0(3)$ & $\mathrm{O}(50)-\mathrm{U}(8)-\mathrm{K}(1)$ & $44.0(3)$ \\
\hline $\mathrm{O}(15)-\mathrm{U}(8)-\mathrm{K}(1)$ & $54.8(3)$ & $\mathrm{O}(40)-U(8)-K(2)$ & $123.0(4)$ \\
\hline $\mathrm{O}(41)-\mathrm{U}(8)-\mathrm{K}(2)$ & $55.7(4)$ & $\mathrm{O}(39)-U(8)-K(2)$ & $80.8(3)$ \\
\hline $\mathrm{O}(13)-\mathrm{U}(8)-\mathrm{K}(2)$ & $136.6(3)$ & $\mathrm{O}(86)-\mathrm{U}(8)-\mathrm{K}(2)$ & $42.8(3)$ \\
\hline$O(35)-U(8)-K(2)$ & $52.2(3)$ & $\mathrm{O}(50)-\mathrm{U}(8)-\mathrm{K}(2)$ & $99.9(3)$ \\
\hline $\mathrm{O}(15)-\mathrm{U}(8)-\mathrm{K}(2)$ & $147.7(4)$ & $K(1)-U(8)-K(2)$ & $97.4(3)$ \\
\hline$O(54)-U(9)-O(56)$ & $177.2(5)$ & $\mathrm{O}(54)-U(9)-O(52) \# 1$ & $92.1(4)$ \\
\hline $\mathrm{O}(56)-\mathrm{U}(9)-\mathrm{O}(52) \# 1$ & $90.5(4)$ & $\mathrm{O}(54)-\mathrm{U}(9)-\mathrm{O}(10) \# 1$ & $92.4(4)$ \\
\hline $\mathrm{O}(56)-U(9)-O(10) \# 1$ & $89.5(4)$ & $\mathrm{O}(52) \# 1-\mathrm{U}(9)-\mathrm{O}(10) \# 1$ & $68.3(3)$ \\
\hline $\mathrm{O}(54)-U(9)-O(42) \# 1$ & $87.9(4)$ & $\mathrm{O}(56)-\mathrm{U}(9)-\mathrm{O}(42) \# 1$ & $92.4(4)$ \\
\hline $\mathrm{O}(52) \# 1-\mathrm{U}(9)-\mathrm{O}(42) \# 1$ & $104.3(3)$ & $\mathrm{O}(10) \# 1-\mathrm{U}(9)-\mathrm{O}(42) \# 1$ & $36.2(3)$ \\
\hline $\mathrm{O}(54)-U(9)-O(55) \# 1$ & $91.7(4)$ & $\mathrm{O}(56)-\mathrm{U}(9)-\mathrm{O}(55) \# 1$ & $89.8(4)$ \\
\hline $\mathrm{O}(52) \# 1-U(9)-O(55) \# 1$ & $36.5(3)$ & $\mathrm{O}(10) \# 1-\mathrm{U}(9)-\mathrm{O}(55) \# 1$ & $104.7(3)$ \\
\hline $\mathrm{O}(42) \# 1-\mathrm{U}(9)-\mathrm{O}(55) \# 1$ & $140.8(3)$ & $\mathrm{O}(54)-\mathrm{U}(9)-\mathrm{O}(51)$ & $86.9(4)$ \\
\hline$O(56)-U(9)-O(51)$ & $91.3(4)$ & $\mathrm{O}(52) \# 1-U(9)-O(51)$ & $108.6(3)$ \\
\hline $\mathrm{O}(10) \# 1-U(9)-O(51)$ & $176.7(4)$ & $\mathrm{O}(42) \# 1-\mathrm{U}(9)-\mathrm{O}(51)$ & $146.9(4)$ \\
\hline $\mathrm{O}(55) \# 1-U(9)-O(51)$ & $72.1(3)$ & $O(54)-U(9)-O(53)$ & $89.6(4)$ \\
\hline $\mathrm{O}(56)-\mathrm{U}(9)-\mathrm{O}(53)$ & $87.9(4)$ & $\mathrm{O}(52) \# 1-U(9)-O(53)$ & $177.6(4)$ \\
\hline $\mathrm{O}(10) \# 1-U(9)-O(53)$ & $110.0(3)$ & $\mathrm{O}(42) \# 1-U(9)-\mathrm{O}(53)$ & $74.1(3)$ \\
\hline $\mathrm{O}(55) \# 1-U(9)-O(53)$ & $145.1(3)$ & $O(51)-U(9)-O(53)$ & $73.2(3)$ \\
\hline $\mathrm{O}(54)-\mathrm{U}(9)-\mathrm{Na}(11) \# 1$ & $23.7(3)$ & $\mathrm{O}(56)-\mathrm{U}(9)-\mathrm{Na}(11) \# 1$ & $155.0(4)$ \\
\hline $\mathrm{O}(52) \# 1-\mathrm{U}(9)-\mathrm{Na}(11) \# 1$ & $108.5(3)$ & $\mathrm{O}(10) \# 1-\mathrm{U}(9)-\mathrm{Na}(11) \# 1$ & $83.1(3)$ \\
\hline $\mathrm{O}(42) \# 1-\mathrm{U}(9)-\mathrm{Na}(11) \# 1$ & $67.8(3)$ & $\mathrm{O}(55) \# 1-\mathrm{U}(9)-\mathrm{Na}(11) \# 1$ & $115.1(3)$ \\
\hline $\mathrm{O}(51)-\mathrm{U}(9)-\mathrm{Na}(11) \# 1$ & $97.5(3)$ & $\mathrm{O}(53)-\mathrm{U}(9)-\mathrm{Na}(11) \# 1$ & $72.6(3)$ \\
\hline$O(59)-U(10)-O(60)$ & $178.4(4)$ & $\mathrm{O}(59)-U(10)-O(39)$ & $92.3(4)$ \\
\hline$O(60)-U(10)-O(39)$ & $87.8(4)$ & $\mathrm{O}(59)-\mathrm{U}(10)-\mathrm{O}(52)$ & $92.4(4)$ \\
\hline$O(60)-U(10)-O(52)$ & $89.1(4)$ & $\mathrm{O}(39)-\mathrm{U}(10)-\mathrm{O}(52)$ & $70.4(3)$ \\
\hline$O(59)-U(10)-O(57)$ & $90.6(4)$ & $\mathrm{O}(60)-\mathrm{U}(10)-\mathrm{O}(57)$ & $87.9(4)$ \\
\hline $\mathrm{O}(39)-U(10)-O(57)$ & $106.4(3)$ & $\mathrm{O}(52)-\mathrm{U}(10)-\mathrm{O}(57)$ & $175.7(4)$ \\
\hline$O(59)-U(10)-O(35)$ & $88.9(4)$ & $\mathrm{O}(60)-\mathrm{U}(10)-\mathrm{O}(35)$ & $90.3(4)$ \\
\hline
\end{tabular}




\begin{tabular}{|c|c|c|c|}
\hline$O(39)-U(10)-O(35)$ & $35.9(2)$ & $O(52)-U(10)-O(35)$ & $106.2(3)$ \\
\hline$O(57)-U(10)-O(35)$ & $70.7(3)$ & $\mathrm{O}(59)-\mathrm{U}(10)-\mathrm{O}(55)$ & $90.6(4)$ \\
\hline$O(60)-U(10)-O(55)$ & $90.8(4)$ & $\mathrm{O}(39)-\mathrm{U}(10)-\mathrm{O}(55)$ & $106.4(3)$ \\
\hline$O(52)-U(10)-O(55)$ & $36.1(3)$ & $\mathrm{O}(57)-\mathrm{U}(10)-\mathrm{O}(55)$ & $147.0(4)$ \\
\hline$O(35)-U(10)-O(55)$ & $142.3(3)$ & $O(59)-U(10)-O(58)$ & $92.5(4)$ \\
\hline $\mathrm{O}(60)-\mathrm{U}(10)-\mathrm{O}(58)$ & $87.5(4)$ & $\mathrm{O}(39)-\mathrm{U}(10)-\mathrm{O}(58)$ & $174.4(4)$ \\
\hline$O(52)-U(10)-O(58)$ & $106.6(3)$ & $\mathrm{O}(57)-\mathrm{U}(10)-\mathrm{O}(58)$ & $76.3(3)$ \\
\hline$O(35)-U(10)-O(58)$ & $147.0(3)$ & $O(55)-U(10)-O(58)$ & $70.7(3)$ \\
\hline $\mathrm{O}(59)-\mathrm{U}(10)-\mathrm{Na}(13)$ & $162.0(4)$ & $\mathrm{O}(60)-\mathrm{U}(10)-\mathrm{Na}(13)$ & $19.0(3)$ \\
\hline $\mathrm{O}(39)-\mathrm{U}(10)-\mathrm{Na}(13)$ & $101.1(3)$ & $\mathrm{O}(52)-\mathrm{U}(10)-\mathrm{Na}(13)$ & $81.1(3)$ \\
\hline $\mathrm{O}(57)-\mathrm{U}(10)-\mathrm{Na}(13)$ & $96.9(3)$ & $\mathrm{O}(35)-\mathrm{U}(10)-\mathrm{Na}(13)$ & $109.0(3)$ \\
\hline $\mathrm{O}(55)-\mathrm{U}(10)-\mathrm{Na}(13)$ & $74.2(3)$ & $\mathrm{O}(58)-\mathrm{U}(10)-\mathrm{Na}(13)$ & $73.6(3)$ \\
\hline $\mathrm{O}(59)-\mathrm{U}(10)-\mathrm{K}(2)$ & $52.4(4)$ & $O(60)-U(10)-K(2)$ & $126.1(4)$ \\
\hline $\mathrm{O}(39)-U(10)-\mathrm{K}(2)$ & $87.2(3)$ & $O(52)-U(10)-K(2)$ & $138.1(3)$ \\
\hline $\mathrm{O}(57)-\mathrm{U}(10)-\mathrm{K}(2)$ & $43.2(3)$ & $\mathrm{O}(35)-U(10)-\mathrm{K}(2)$ & $57.8(3)$ \\
\hline $\mathrm{O}(55)-U(10)-K(2)$ & $141.6(4)$ & $\mathrm{O}(58)-\mathrm{U}(10)-\mathrm{K}(2)$ & $97.9(3)$ \\
\hline $\mathrm{Na}(13)-\mathrm{U}(10)-\mathrm{K}(2)$ & $139.3(4)$ & $\mathrm{O}(61)-\mathrm{U}(11)-\mathrm{O}(87)$ & $177.4(5)$ \\
\hline $\mathrm{O}(61)-\mathrm{U}(11)-\mathrm{O}(4)$ & $92.6(4)$ & $\mathrm{O}(87)-\mathrm{U}(11)-\mathrm{O}(4)$ & $89.0(4)$ \\
\hline$O(61)-U(11)-O(63)$ & $93.2(4)$ & $O(87)-U(11)-O(63)$ & $89.2(4)$ \\
\hline$O(4)-U(11)-O(63)$ & $69.9(3)$ & $O(61)-U(11)-O(64)$ & $90.3(4)$ \\
\hline $\mathrm{O}(87)-\mathrm{U}(11)-\mathrm{O}(64)$ & $91.1(4)$ & $\mathrm{O}(4)-\mathrm{U}(11)-\mathrm{O}(64)$ & $106.3(3)$ \\
\hline$O(63)-U(11)-O(64)$ & $36.4(3)$ & $O(61)-U(11)-O(2)$ & $89.0(4)$ \\
\hline $\mathrm{O}(87)-\mathrm{U}(11)-\mathrm{O}(2)$ & $91.2(4)$ & $\mathrm{O}(4)-\mathrm{U}(11)-\mathrm{O}(2)$ & $36.0(3)$ \\
\hline$O(63)-U(11)-O(2)$ & $105.9(3)$ & $O(64)-U(11)-O(2)$ & $142.1(4)$ \\
\hline$O(61)-U(11)-O(65)$ & $86.8(4)$ & $\mathrm{O}(87)-\mathrm{U}(11)-\mathrm{O}(65)$ & $90.7(4)$ \\
\hline $\mathrm{O}(4)-\mathrm{U}(11)-\mathrm{O}(65)$ & $109.0(4)$ & $O(63)-U(11)-O(65)$ & $178.9(4)$ \\
\hline$O(64)-U(11)-O(65)$ & $144.7(4)$ & $\mathrm{O}(2)-\mathrm{U}(11)-\mathrm{O}(65)$ & $73.0(3)$ \\
\hline$O(61)-U(11)-O(62)$ & $89.1(4)$ & $O(87)-U(11)-O(62)$ & $89.3(4)$ \\
\hline$O(4)-U(11)-O(62)$ & $178.0(4)$ & $O(63)-U(11)-O(62)$ & $109.1(3)$ \\
\hline$O(64)-U(11)-O(62)$ & $72.8(3)$ & $O(2)-U(11)-O(62)$ & $145.0(4)$ \\
\hline$O(65)-U(11)-O(62)$ & $72.0(3)$ & $\mathrm{O}(61)-\mathrm{U}(11)-\mathrm{Na}(7)$ & $25.3(3)$ \\
\hline $\mathrm{O}(87)-\mathrm{U}(11)-\mathrm{Na}(7)$ & $154.5(4)$ & $\mathrm{O}(4)-\mathrm{U}(11)-\mathrm{Na}(7)$ & $112.7(3)$ \\
\hline $\mathrm{O}(63)-\mathrm{U}(11)-\mathrm{Na}(7)$ & $86.1(3)$ & $\mathrm{O}(64)-\mathrm{U}(11)-\mathrm{Na}(7)$ & $70.5(3)$ \\
\hline $\mathrm{O}(2)-\mathrm{U}(11)-\mathrm{Na}(7)$ & $114.2(3)$ & $\mathrm{O}(65)-\mathrm{U}(11)-\mathrm{Na}(7)$ & $94.3(3)$ \\
\hline $\mathrm{O}(62)-\mathrm{U}(11)-\mathrm{Na}(7)$ & $68.7(3)$ & $\mathrm{O}(61)-\mathrm{U}(11)-\mathrm{Na}(16)$ & $162.0(3)$ \\
\hline $\mathrm{O}(87)-\mathrm{U}(11)-\mathrm{Na}(16)$ & $20.6(3)$ & $\mathrm{O}(4)-\mathrm{U}(11)-\mathrm{Na}(16)$ & $73.4(2)$ \\
\hline $\mathrm{O}(63)-\mathrm{U}(11)-\mathrm{Na}(16)$ & $71.6(2)$ & $\mathrm{O}(64)-\mathrm{U}(11)-\mathrm{Na}(16)$ & $83.1(2)$ \\
\hline $\mathrm{O}(2)-\mathrm{U}(11)-\mathrm{Na}(16)$ & $86.1(2)$ & $\mathrm{O}(65)-\mathrm{U}(11)-\mathrm{Na}(16)$ & $108.2(3)$ \\
\hline $\mathrm{O}(62)-\mathrm{U}(11)-\mathrm{Na}(16)$ & $104.7(2)$ & $\mathrm{Na}(7)-\mathrm{U}(11)-\mathrm{Na}(16)$ & $153.6(2)$ \\
\hline$O(66)-U(12)-O(69)$ & $178.5(5)$ & $\mathrm{O}(66)-\mathrm{U}(12)-\mathrm{O}(33)$ & $90.4(4)$ \\
\hline$O(69)-U(12)-O(33)$ & $90.5(4)$ & $\mathrm{O}(66)-\mathrm{U}(12)-\mathrm{O}(63)$ & $92.7(4)$ \\
\hline$O(69)-U(12)-O(63)$ & $88.7(4)$ & $O(33)-U(12)-O(63)$ & $71.8(3)$ \\
\hline$O(66)-U(12)-O(68)$ & $89.2(4)$ & $\mathrm{O}(69)-\mathrm{U}(12)-\mathrm{O}(68)$ & $89.4(4)$ \\
\hline $\mathrm{O}(33)-\mathrm{U}(12)-\mathrm{O}(68)$ & $106.0(3)$ & $\mathrm{O}(63)-\mathrm{U}(12)-\mathrm{O}(68)$ & $177.2(4)$ \\
\hline
\end{tabular}




\begin{tabular}{|c|c|c|c|}
\hline$O(66)-U(12)-O(64)$ & $89.0(4)$ & $O(69)-U(12)-O(64)$ & $91.9(4)$ \\
\hline$O(33)-U(12)-O(64)$ & $107.9(3)$ & $O(63)-U(12)-O(64)$ & $36.3(3)$ \\
\hline$O(68)-U(12)-O(64)$ & $146.0(4)$ & $O(66)-U(12)-O(67)$ & $88.9(4)$ \\
\hline$O(69)-U(12)-O(67)$ & $90.3(4)$ & $\mathrm{O}(33)-\mathrm{U}(12)-\mathrm{O}(67)$ & $178.5(4)$ \\
\hline $\mathrm{O}(63)-\mathrm{U}(12)-\mathrm{O}(67)$ & $106.9(3)$ & $\mathrm{O}(68)-\mathrm{U}(12)-\mathrm{O}(67)$ & $75.3(3)$ \\
\hline$O(64)-U(12)-O(67)$ & $70.7(3)$ & $O(66)-U(12)-O(49)$ & $87.1(4)$ \\
\hline$O(69)-U(12)-O(49)$ & $92.9(4)$ & $\mathrm{O}(33)-\mathrm{U}(12)-\mathrm{O}(49)$ & $35.7(3)$ \\
\hline $\mathrm{O}(63)-\mathrm{U}(12)-\mathrm{O}(49)$ & $107.4(3)$ & $O(68)-U(12)-O(49)$ & $70.5(3)$ \\
\hline$O(64)-U(12)-O(49)$ & $143.3(4)$ & $\mathrm{O}(67)-\mathrm{U}(12)-\mathrm{O}(49)$ & $145.6(4)$ \\
\hline $\mathrm{O}(66)-\mathrm{U}(12)-\mathrm{K}(3)$ & $49.7(4)$ & $\mathrm{O}(69)-U(12)-\mathrm{K}(3)$ & $129.3(5)$ \\
\hline $\mathrm{O}(33)-\mathrm{U}(12)-\mathrm{K}(3)$ & $84.1(3)$ & $\mathrm{O}(63)-\mathrm{U}(12)-\mathrm{K}(3)$ & $135.5(4)$ \\
\hline $\mathrm{O}(68)-\mathrm{U}(12)-\mathrm{K}(3)$ & $45.1(3)$ & $\mathrm{O}(64)-U(12)-\mathrm{K}(3)$ & $137.7(4)$ \\
\hline $\mathrm{O}(67)-\mathrm{U}(12)-\mathrm{K}(3)$ & $96.4(3)$ & $\mathrm{O}(49)-\mathrm{U}(12)-\mathrm{K}(3)$ & $56.2(3)$ \\
\hline $\mathrm{O}(66)-\mathrm{U}(12)-\mathrm{Na}(7)$ & $23.5(3)$ & $\mathrm{O}(69)-\mathrm{U}(12)-\mathrm{Na}(7)$ & $156.4(4)$ \\
\hline $\mathrm{O}(33)-\mathrm{U}(12)-\mathrm{Na}(7)$ & 109.7(3) & $\mathrm{O}(63)-\mathrm{U}(12)-\mathrm{Na}(7)$ & $86.3(3)$ \\
\hline $\mathrm{O}(68)-\mathrm{U}(12)-\mathrm{Na}(7)$ & $96.2(3)$ & $\mathrm{O}(64)-\mathrm{U}(12)-\mathrm{Na}(7)$ & $70.8(3)$ \\
\hline $\mathrm{O}(67)-\mathrm{U}(12)-\mathrm{Na}(7)$ & $69.2(3)$ & $\mathrm{O}(49)-\mathrm{U}(12)-\mathrm{Na}(7)$ & $110.7(3)$ \\
\hline $\mathrm{K}(3)-\mathrm{U}(12)-\mathrm{Na}(7)$ & $67.0(4)$ & $O(2 \mathrm{I})-\mathrm{I}(1)-\mathrm{O}(1 \mathrm{I})$ & $102.1(6)$ \\
\hline$O(2 I)-I(1)-O(3 I)$ & $101.3(7)$ & $O(1 I)-I(1)-O(3 I)$ & $98.9(7)$ \\
\hline $\mathrm{O}(2 \mathrm{I})-\mathrm{I}(1)-\mathrm{Na}(3)$ & $121.3(5)$ & $\mathrm{O}(1 \mathrm{I})-\mathrm{I}(1)-\mathrm{Na}(3)$ & $122.1(6)$ \\
\hline $\mathrm{O}(3 \mathrm{I})-\mathrm{I}(1)-\mathrm{Na}(3)$ & $40.6(4)$ & $\mathrm{O}(4 \mathrm{~W})-\mathrm{K}(1)-\mathrm{O}(25)$ & $129.3(6)$ \\
\hline $\mathrm{O}(4 \mathrm{~W})-\mathrm{K}(1)-\mathrm{O}(50)$ & $75.5(4)$ & $\mathrm{O}(25)-\mathrm{K}(1)-\mathrm{O}(50)$ & $150.6(6)$ \\
\hline $\mathrm{O}(4 \mathrm{~W})-\mathrm{K}(1)-\mathrm{O}(85)$ & $73.4(4)$ & $\mathrm{O}(25)-\mathrm{K}(1)-\mathrm{O}(85)$ & $60.3(4)$ \\
\hline $\mathrm{O}(50)-\mathrm{K}(1)-\mathrm{O}(85)$ & $148.3(5)$ & $\mathrm{O}(4 \mathrm{~W})-\mathrm{K}(1)-\mathrm{O}(12)$ & $161.9(6)$ \\
\hline $\mathrm{O}(25)-\mathrm{K}(1)-\mathrm{O}(12)$ & $55.3(3)$ & $\mathrm{O}(50)-\mathrm{K}(1)-\mathrm{O}(12)$ & $96.1(5)$ \\
\hline $\mathrm{O}(85)-\mathrm{K}(1)-\mathrm{O}(12)$ & $115.5(5)$ & $O(4 W)-K(1)-H(15 A) \# 2$ & $101.7(6)$ \\
\hline $\mathrm{O}(25)-\mathrm{K}(1)-\mathrm{H}(15 \mathrm{~A}) \# 2$ & $113.7(6)$ & $\mathrm{O}(50)-\mathrm{K}(1)-\mathrm{H}(15 \mathrm{~A}) \# 2$ & $68.1(6)$ \\
\hline $\mathrm{O}(85)-\mathrm{K}(1)-\mathrm{H}(15 \mathrm{~A}) \# 2$ & $112.5(6)$ & $\mathrm{O}(12)-\mathrm{K}(1)-\mathrm{H}(15 \mathrm{~A}) \# 2$ & $89.6(6)$ \\
\hline $\mathrm{O}(4 \mathrm{~W})-\mathrm{K}(1)-\mathrm{O}(11)$ & $134.4(6)$ & $\mathrm{O}(25)-\mathrm{K}(1)-\mathrm{O}(11)$ & $75.4(4)$ \\
\hline $\mathrm{O}(50)-\mathrm{K}(1)-\mathrm{O}(11)$ & $98.5(5)$ & $\mathrm{O}(85)-\mathrm{K}(1)-\mathrm{O}(11)$ & $98.8(5)$ \\
\hline $\mathrm{O}(12)-\mathrm{K}(1)-\mathrm{O}(11)$ & $62.0(3)$ & $\mathrm{H}(15 \mathrm{~A}) \# 2-\mathrm{K}(1)-\mathrm{O}(11)$ & $38.8(5)$ \\
\hline $\mathrm{O}(4 \mathrm{~W})-\mathrm{K}(1)-\mathrm{O}(76 \mathrm{~W})$ & $77.1(4)$ & $\mathrm{O}(25)-\mathrm{K}(1)-\mathrm{O}(76 \mathrm{~W})$ & $66.2(4)$ \\
\hline$O(50)-K(1)-O(76 W)$ & $113.1(5)$ & $\mathrm{O}(85)-\mathrm{K}(1)-\mathrm{O}(76 \mathrm{~W})$ & $65.5(4)$ \\
\hline$O(12)-K(1)-O(76 W)$ & $92.1(4)$ & $\mathrm{H}(15 \mathrm{~A}) \# 2-\mathrm{K}(1)-\mathrm{O}(76 \mathrm{~W})$ & $177.8(7)$ \\
\hline$O(11)-K(1)-O(76 W)$ & $141.5(5)$ & $\mathrm{O}(4 \mathrm{~W})-\mathrm{K}(1)-\mathrm{O}(23)$ & $110.1(5)$ \\
\hline $\mathrm{O}(25)-\mathrm{K}(1)-\mathrm{O}(23)$ & $56.0(3)$ & $\mathrm{O}(50)-\mathrm{K}(1)-\mathrm{O}(23)$ & $137.8(6)$ \\
\hline $\mathrm{O}(85)-\mathrm{K}(1)-\mathrm{O}(23)$ & $52.1(3)$ & $\mathrm{O}(12)-\mathrm{K}(1)-\mathrm{O}(23)$ & $87.0(4)$ \\
\hline $\mathrm{H}(15 \mathrm{~A}) \# 2-\mathrm{K}(1)-\mathrm{O}(23)$ & $69.8(5)$ & $\mathrm{O}(11)-\mathrm{K}(1)-\mathrm{O}(23)$ & $46.6(3)$ \\
\hline $\mathrm{O}(76 \mathrm{~W})-\mathrm{K}(1)-\mathrm{O}(23)$ & $108.9(5)$ & $\mathrm{O}(4 \mathrm{~W})-\mathrm{K}(1)-\mathrm{O}(41)$ & $110.7(5)$ \\
\hline $\mathrm{O}(25)-\mathrm{K}(1)-\mathrm{O}(41)$ & $97.8(4)$ & $\mathrm{O}(50)-\mathrm{K}(1)-\mathrm{O}(41)$ & $54.3(4)$ \\
\hline $\mathrm{O}(85)-\mathrm{K}(1)-\mathrm{O}(41)$ & $145.8(6)$ & $\mathrm{O}(12)-\mathrm{K}(1)-\mathrm{O}(41)$ & $52.8(3)$ \\
\hline $\mathrm{H}(15 \mathrm{~A}) \# 2-\mathrm{K}(1)-\mathrm{O}(41)$ & $100.2(6)$ & $\mathrm{O}(11)-\mathrm{K}(1)-\mathrm{O}(41)$ & $100.5(5)$ \\
\hline$O(76 W)-K(1)-O(41)$ & $82.0(4)$ & $\mathrm{O}(23)-\mathrm{K}(1)-\mathrm{O}(41)$ & $139.2(5)$ \\
\hline$O(4 W)-K(1)-O(15 W) \# 2$ & $85.4(4)$ & $\mathrm{O}(25)-\mathrm{K}(1)-\mathrm{O}(15 \mathrm{~W}) \# 2$ & $126.5(5)$ \\
\hline
\end{tabular}




\begin{tabular}{|c|c|c|c|}
\hline $\mathrm{O}(50)-\mathrm{K}(1)-\mathrm{O}(15 \mathrm{~W}) \# 2$ & $62.9(4)$ & $\mathrm{O}(85)-\mathrm{K}(1)-\mathrm{O}(15 \mathrm{~W}) \# 2$ & $108.2(5)$ \\
\hline $\mathrm{O}(12)-\mathrm{K}(1)-\mathrm{O}(15 \mathrm{~W}) \# 2$ & $105.3(5)$ & $\mathrm{H}(15 \mathrm{~A}) \# 2-\mathrm{K}(1)-\mathrm{O}(15 \mathrm{~W}) \# 2$ & $16.3(5)$ \\
\hline $\mathrm{O}(11)-\mathrm{K}(1)-\mathrm{O}(15 \mathrm{~W}) \# 2$ & $53.7(3)$ & $\mathrm{O}(76 \mathrm{~W})-\mathrm{K}(1)-\mathrm{O}(15 \mathrm{~W}) \# 2$ & $162.4(5)$ \\
\hline $\mathrm{O}(23)-\mathrm{K}(1)-\mathrm{O}(15 \mathrm{~W}) \# 2$ & $75.6(4)$ & $\mathrm{O}(41)-\mathrm{K}(1)-\mathrm{O}(15 \mathrm{~W}) \# 2$ & $106.0(5)$ \\
\hline$O(4 W)-K(1)-O(15)$ & $126.0(5)$ & $\mathrm{O}(25)-\mathrm{K}(1)-\mathrm{O}(15)$ & $104.7(4)$ \\
\hline $\mathrm{O}(50)-\mathrm{K}(1)-\mathrm{O}(15)$ & $53.5(3)$ & $\mathrm{O}(85)-\mathrm{K}(1)-\mathrm{O}(15)$ & $150.6(5)$ \\
\hline $\mathrm{O}(12)-\mathrm{K}(1)-\mathrm{O}(15)$ & $53.7(3)$ & $\mathrm{H}(15 \mathrm{~A}) \# 2-\mathrm{K}(1)-\mathrm{O}(15)$ & $47.1(5)$ \\
\hline $\mathrm{O}(11)-\mathrm{K}(1)-\mathrm{O}(15)$ & $51.8(3)$ & $O(76 W)-K(1)-O(15)$ & $135.1(5)$ \\
\hline $\mathrm{O}(23)-\mathrm{K}(1)-\mathrm{O}(15)$ & $98.5(4)$ & $\mathrm{O}(41)-\mathrm{K}(1)-\mathrm{O}(15)$ & $54.8(3)$ \\
\hline$O(15 W) \# 2-K(1)-O(15)$ & $58.2(3)$ & $O(5 W)-K(2)-O(57)$ & $143.8(7)$ \\
\hline$O(5 W)-K(2)-O(29) \# 1$ & $128.3(6)$ & $\mathrm{O}(57)-\mathrm{K}(2)-\mathrm{O}(29) \# 1$ & $69.0(4)$ \\
\hline$O(5 W)-K(2)-O(31 W) \# 3$ & $84.9(5)$ & $O(57)-K(2)-O(31 W) \# 3$ & $63.6(4)$ \\
\hline $\mathrm{O}(29) \# 1-\mathrm{K}(2)-\mathrm{O}(31 \mathrm{~W}) \# 3$ & $128.6(6)$ & $O(5 W)-K(2)-O(86)$ & $77.7(4)$ \\
\hline $\mathrm{O}(57)-\mathrm{K}(2)-\mathrm{O}(86)$ & $101.5(5)$ & $\mathrm{O}(29) \# 1-\mathrm{K}(2)-\mathrm{O}(86)$ & $148.2(5)$ \\
\hline $\mathrm{O}(31 \mathrm{~W}) \# 3-\mathrm{K}(2)-\mathrm{O}(86)$ & $63.4(4)$ & $\mathrm{O}(5 \mathrm{~W})-\mathrm{K}(2)-\mathrm{H}(31 \mathrm{~B}) \# 3$ & $103.1(6)$ \\
\hline $\mathrm{O}(57)-\mathrm{K}(2)-\mathrm{H}(31 \mathrm{~B}) \# 3$ & $48.3(5)$ & $\mathrm{O}(29) \# 1-\mathrm{K}(2)-\mathrm{H}(31 \mathrm{~B}) \# 3$ & $116.9(6)$ \\
\hline $\mathrm{O}(31 \mathrm{~W}) \# 3-\mathrm{K}(2)-\mathrm{H}(31 \mathrm{~B}) \# 3$ & $18.6(3)$ & $\mathrm{O}(86)-\mathrm{K}(2)-\mathrm{H}(31 \mathrm{~B}) \# 3$ & $64.4(7)$ \\
\hline $\mathrm{O}(5 \mathrm{~W})-\mathrm{K}(2)-\mathrm{O}(19) \# 1$ & $74.1(4)$ & $\mathrm{O}(57)-\mathrm{K}(2)-\mathrm{O}(19) \# 1$ & $97.7(5)$ \\
\hline O(29)\#1-K(2)-O(19)\#1 & $59.7(3)$ & $\mathrm{O}(31 \mathrm{~W}) \# 3-\mathrm{K}(2)-\mathrm{O}(19) \# 1$ & $107.7(5)$ \\
\hline$O(86)-K(2)-O(19) \# 1$ & $151.1(5)$ & $H(31 B) \# 3-K(2)-O(19) \# 1$ & $116.4(7)$ \\
\hline$O(5 W)-K(2)-O(33 W)$ & $74.2(5)$ & $O(57)-K(2)-O(33 W)$ & $134.7(6)$ \\
\hline$O(29) \# 1-K(2)-O(33 W)$ & $66.2(4)$ & $\mathrm{O}(31 \mathrm{~W}) \# 3-\mathrm{K}(2)-\mathrm{O}(33 \mathrm{~W})$ & $159.0(6)$ \\
\hline$O(86)-K(2)-O(33 W)$ & $113.3(6)$ & $H(31 B) \# 3-K(2)-O(33 W)$ & $176.9(7)$ \\
\hline $\mathrm{O}(19) \# 1-K(2)-O(33 W)$ & $64.4(4)$ & $O(5 W)-K(2)-H(33 B)$ & $89.4(7)$ \\
\hline $\mathrm{O}(57)-\mathrm{K}(2)-\mathrm{H}(33 \mathrm{~B})$ & $123.7(8)$ & $\mathrm{O}(29) \# 1-\mathrm{K}(2)-\mathrm{H}(33 \mathrm{~B})$ & $60.1(8)$ \\
\hline $\mathrm{O}(31 \mathrm{~W}) \# 3-\mathrm{K}(2)-\mathrm{H}(33 \mathrm{~B})$ & $171.3(9)$ & $\mathrm{O}(86)-\mathrm{K}(2)-\mathrm{H}(33 \mathrm{~B})$ & $108.9(8)$ \\
\hline$H(31 B) \# 3-K(2)-H(33 B)$ & $163.5(7)$ & $\mathrm{O}(19) \# 1-\mathrm{K}(2)-\mathrm{H}(33 \mathrm{~B})$ & $77.0(7)$ \\
\hline $\mathrm{O}(33 \mathrm{~W})-\mathrm{K}(2)-\mathrm{H}(33 \mathrm{~B})$ & $17.8(3)$ & $\mathrm{O}(5 \mathrm{~W})-\mathrm{K}(2)-\mathrm{H}(5 \mathrm{~B})$ & $17.6(4)$ \\
\hline $\mathrm{O}(57)-\mathrm{K}(2)-\mathrm{H}(5 \mathrm{~B})$ & $140.0(7)$ & $\mathrm{O}(29) \# 1-\mathrm{K}(2)-\mathrm{H}(5 \mathrm{~B})$ & $144.9(7)$ \\
\hline$O(31 W) \# 3-K(2)-H(5 B)$ & $76.5(5)$ & $\mathrm{O}(86)-\mathrm{K}(2)-\mathrm{H}(5 \mathrm{~B})$ & $60.1(4)$ \\
\hline$H(31 B) \# 3-K(2)-H(5 B)$ & $92.9(6)$ & $\mathrm{O}(19) \# 1-\mathrm{K}(2)-\mathrm{H}(5 \mathrm{~B})$ & $91.5(5)$ \\
\hline$O(33 W)-K(2)-H(5 B)$ & $84.1(5)$ & $H(33 B)-K(2)-H(5 B)$ & $96.3(8)$ \\
\hline $\mathrm{O}(5 \mathrm{~W})-\mathrm{K}(2)-\mathrm{O}(59)$ & $156.2(6)$ & $\mathrm{O}(57)-\mathrm{K}(2)-\mathrm{O}(59)$ & $59.5(3)$ \\
\hline$O(29) \# 1-K(2)-O(59)$ & $54.5(3)$ & $\mathrm{O}(31 \mathrm{~W}) \# 3-\mathrm{K}(2)-\mathrm{O}(59)$ & $111.6(5)$ \\
\hline $\mathrm{O}(86)-\mathrm{K}(2)-\mathrm{O}(59)$ & $94.2(4)$ & $\mathrm{H}(31 \mathrm{~B}) \# 3-\mathrm{K}(2)-\mathrm{O}(59)$ & $93.1(5)$ \\
\hline $\mathrm{O}(19) \# 1-\mathrm{K}(2)-\mathrm{O}(59)$ & $114.2(5)$ & $\mathrm{O}(33 \mathrm{~W})-\mathrm{K}(2)-\mathrm{O}(59)$ & $89.1(5)$ \\
\hline $\mathrm{H}(33 \mathrm{~B})-\mathrm{K}(2)-\mathrm{O}(59)$ & $71.9(5)$ & $H(5 B)-K(2)-O(59)$ & $147.3(6)$ \\
\hline$O(5 W)-K(2)-O(35)$ & $129.2(5)$ & $\mathrm{O}(57)-\mathrm{K}(2)-\mathrm{O}(35)$ & $52.4(3)$ \\
\hline$O(29) \# 1-K(2)-O(35)$ & $102.3(4)$ & $\mathrm{O}(31 \mathrm{~W}) \# 3-\mathrm{K}(2)-\mathrm{O}(35)$ & $62.9(4)$ \\
\hline $\mathrm{O}(86)-\mathrm{K}(2)-\mathrm{O}(35)$ & $53.5(3)$ & $\mathrm{H}(31 \mathrm{~B}) \# 3-\mathrm{K}(2)-\mathrm{O}(35)$ & $46.9(5)$ \\
\hline $\mathrm{O}(19) \# 1-\mathrm{K}(2)-\mathrm{O}(35)$ & $150.0(5)$ & $O(33 W)-K(2)-O(35)$ & $133.8(5)$ \\
\hline $\mathrm{H}(33 \mathrm{~B})-\mathrm{K}(2)-\mathrm{O}(35)$ & $116.7(6)$ & $\mathrm{H}(5 \mathrm{~B})-\mathrm{K}(2)-\mathrm{O}(35)$ & $111.9(5)$ \\
\hline $\mathrm{O}(59)-\mathrm{K}(2)-\mathrm{O}(35)$ & $53.6(3)$ & $O(7 W)-K(3)-H(59 D)$ & $41.3(6)$ \\
\hline $\mathrm{O}(7 \mathrm{~W})-\mathrm{K}(3)-\mathrm{O}(68)$ & $148.4(9)$ & $H(59 D)-K(3)-O(68)$ & $158.0(8)$ \\
\hline
\end{tabular}




\begin{tabular}{|c|c|c|c|}
\hline$O(7 W)-K(3)-O(77 W)$ & $74.9(6)$ & $H(59 D)-K(3)-O(77 W)$ & $115.6(8)$ \\
\hline$O(68)-K(3)-O(77 W)$ & $76.1(6)$ & $\mathrm{O}(7 \mathrm{~W})-\mathrm{K}(3)-\mathrm{O}(36)$ & 119.1(6) \\
\hline $\mathrm{H}(59 \mathrm{D})-\mathrm{K}(3)-\mathrm{O}(36)$ & $86.0(7)$ & $\mathrm{O}(68)-\mathrm{K}(3)-\mathrm{O}(36)$ & $72.9(4)$ \\
\hline$O(77 W)-K(3)-O(36)$ & $131.3(6)$ & $O(7 W)-K(3)-O(32)$ & $79.1(4)$ \\
\hline $\mathrm{H}(59 \mathrm{D})-\mathrm{K}(3)-\mathrm{O}(32)$ & $100.3(7)$ & $\mathrm{O}(68)-K(3)-O(32)$ & $101.5(6)$ \\
\hline$O(77 W)-K(3)-O(32)$ & $68.3(5)$ & $\mathrm{O}(36)-\mathrm{K}(3)-\mathrm{O}(32)$ & $154.3(7)$ \\
\hline$O(7 W)-K(3)-H(7 A)$ & $17.8(4)$ & $H(59 D)-K(3)-H(7 A)$ & $51.4(6)$ \\
\hline $\mathrm{O}(68)-\mathrm{K}(3)-\mathrm{H}(7 \mathrm{~A})$ & $146.2(9)$ & $O(77 W)-K(3)-H(7 A)$ & $70.5(6)$ \\
\hline$O(36)-K(3)-H(7 A)$ & $135.3(7)$ & $\mathrm{O}(32)-K(3)-\mathrm{H}(7 \mathrm{~A})$ & $61.2(5)$ \\
\hline $\mathrm{O}(7 \mathrm{~W})-\mathrm{K}(3)-\mathrm{O}(66)$ & $151.4(8)$ & $H(59 D)-K(3)-O(66)$ & $112.6(8)$ \\
\hline $\mathrm{O}(68)-\mathrm{K}(3)-\mathrm{O}(66)$ & $60.2(4)$ & $O(77 W)-K(3)-O(66)$ & $131.7(7)$ \\
\hline$O(36)-K(3)-O(66)$ & $55.3(4)$ & $\mathrm{O}(32)-\mathrm{K}(3)-\mathrm{O}(66)$ & $99.8(5)$ \\
\hline$H(7 A)-K(3)-O(66)$ & $146.0(7)$ & $\mathrm{O}(7 \mathrm{~W})-\mathrm{K}(3)-\mathrm{O}(30)$ & $70.3(4)$ \\
\hline$H(59 D)-K(3)-O(30)$ & $65.0(8)$ & $\mathrm{O}(68)-\mathrm{K}(3)-\mathrm{O}(30)$ & $97.7(5)$ \\
\hline$O(77 W)-K(3)-O(30)$ & $90.3(6)$ & $\mathrm{O}(36)-\mathrm{K}(3)-\mathrm{O}(30)$ & $58.5(4)$ \\
\hline $\mathrm{O}(32)-\mathrm{K}(3)-\mathrm{O}(30)$ & $146.4(6)$ & $H(7 A)-K(3)-O(30)$ & $87.8(6)$ \\
\hline $\mathrm{O}(66)-\mathrm{K}(3)-\mathrm{O}(30)$ & $113.7(5)$ & $O(7 W)-K(3)-O(59 V)$ & $58.3(5)$ \\
\hline$H(59 D)-K(3)-O(59 V)$ & $17.2(3)$ & $\mathrm{O}(68)-\mathrm{K}(3)-\mathrm{O}(59 \mathrm{~V})$ & $143.6(7)$ \\
\hline $\mathrm{O}(77 \mathrm{~W})-\mathrm{K}(3)-\mathrm{O}(59 \mathrm{~V})$ & $131.7(7)$ & $\mathrm{O}(36)-\mathrm{K}(3)-\mathrm{O}(59 \mathrm{~V})$ & $70.8(5)$ \\
\hline$O(32)-K(3)-O(59 V)$ & $110.4(6)$ & $H(7 A)-K(3)-O(59 V)$ & $68.2(6)$ \\
\hline $\mathrm{O}(66)-\mathrm{K}(3)-\mathrm{O}(59 \mathrm{~V})$ & $96.6(7)$ & $\mathrm{O}(30)-\mathrm{K}(3)-\mathrm{O}(59 \mathrm{~V})$ & $64.5(5)$ \\
\hline $\mathrm{O}(7 \mathrm{~W})-\mathrm{K}(3)-\mathrm{O}(47)$ & $105.5(6)$ & $H(59 D)-K(3)-O(47)$ & $88.2(8)$ \\
\hline $\mathrm{O}(68)-\mathrm{K}(3)-\mathrm{O}(47)$ & $100.8(5)$ & $\mathrm{O}(77 \mathrm{~W})-\mathrm{K}(3)-\mathrm{O}(47)$ & $123.7(6)$ \\
\hline $\mathrm{O}(36)-\mathrm{K}(3)-\mathrm{O}(47)$ & $98.5(6)$ & $\mathrm{O}(32)-\mathrm{K}(3)-\mathrm{O}(47)$ & $57.2(3)$ \\
\hline$H(7 A)-K(3)-O(47)$ & $93.4(6)$ & $\mathrm{O}(66)-\mathrm{K}(3)-\mathrm{O}(47)$ & $53.6(3)$ \\
\hline $\mathrm{O}(30)-\mathrm{K}(3)-\mathrm{O}(47)$ & $144.3(7)$ & $\mathrm{O}(59 \mathrm{~V})-\mathrm{K}(3)-\mathrm{O}(47)$ & $82.9(6)$ \\
\hline$O(7 W)-K(3)-O(49)$ & $133.3(6)$ & $H(59 D)-K(3)-O(49)$ & $142.1(9)$ \\
\hline $\mathrm{O}(68)-\mathrm{K}(3)-\mathrm{O}(49)$ & $53.9(4)$ & $\mathrm{O}(77 \mathrm{~W})-\mathrm{K}(3)-\mathrm{O}(49)$ & $83.4(6)$ \\
\hline $\mathrm{O}(36)-\mathrm{K}(3)-\mathrm{O}(49)$ & $106.3(5)$ & $O(32)-K(3)-O(49)$ & $54.5(3)$ \\
\hline$H(7 A)-K(3)-O(49)$ & $115.6(6)$ & $\mathrm{O}(66)-\mathrm{K}(3)-\mathrm{O}(49)$ & $55.3(3)$ \\
\hline $\mathrm{O}(30)-\mathrm{K}(3)-\mathrm{O}(49)$ & $151.6(6)$ & $\mathrm{O}(59 \mathrm{~V})-\mathrm{K}(3)-\mathrm{O}(49)$ & $137.4(7)$ \\
\hline $\mathrm{O}(47)-\mathrm{K}(3)-\mathrm{O}(49)$ & $55.0(3)$ & $\mathrm{O}(33 \mathrm{~W})-\mathrm{Na}(1)-\mathrm{O}(5 \mathrm{~W})$ & $95.5(7)$ \\
\hline $\mathrm{O}(33 \mathrm{~W})-\mathrm{Na}(1)-\mathrm{O}(12 \mathrm{~W})$ & $95.9(6)$ & $\mathrm{O}(5 \mathrm{~W})-\mathrm{Na}(1)-\mathrm{O}(12 \mathrm{~W})$ & $166.6(7)$ \\
\hline $\mathrm{O}(33 \mathrm{~W})-\mathrm{Na}(1)-\mathrm{O}(3)$ & $149.7(7)$ & $\mathrm{O}(5 \mathrm{~W})-\mathrm{Na}(1)-\mathrm{O}(3)$ & $89.4(5)$ \\
\hline $\mathrm{O}(12 \mathrm{~W})-\mathrm{Na}(1)-\mathrm{O}(3)$ & $84.4(5)$ & $\mathrm{O}(33 \mathrm{~W})-\mathrm{Na}(1)-\mathrm{O}(43) \# 1$ & $81.9(5)$ \\
\hline $\mathrm{O}(5 \mathrm{~W})-\mathrm{Na}(1)-\mathrm{O}(43) \# 1$ & $81.8(5)$ & $\mathrm{O}(12 \mathrm{~W})-\mathrm{Na}(1)-\mathrm{O}(43) \# 1$ & $106.8(6)$ \\
\hline $\mathrm{O}(3)-\mathrm{Na}(1)-\mathrm{O}(43) \# 1$ & $69.2(4)$ & $\mathrm{O}(33 \mathrm{~W})-\mathrm{Na}(1)-\mathrm{O}(39 \mathrm{~W})$ & $73.3(6)$ \\
\hline $\mathrm{O}(5 \mathrm{~W})-\mathrm{Na}(1)-\mathrm{O}(39 \mathrm{~W})$ & $83.8(5)$ & $\mathrm{O}(12 \mathrm{~W})-\mathrm{Na}(1)-\mathrm{O}(39 \mathrm{~W})$ & $92.8(6)$ \\
\hline $\mathrm{O}(3)-\mathrm{Na}(1)-\mathrm{O}(39 \mathrm{~W})$ & $137.0(7)$ & $\mathrm{O}(43) \# 1-\mathrm{Na}(1)-\mathrm{O}(39 \mathrm{~W})$ & $149.9(7)$ \\
\hline $\mathrm{O}(33 \mathrm{~W})-\mathrm{Na}(1)-\mathrm{O}(61)$ & $137.5(6)$ & $\mathrm{O}(5 \mathrm{~W})-\mathrm{Na}(1)-\mathrm{O}(61)$ & $87.1(5)$ \\
\hline $\mathrm{O}(12 \mathrm{~W})-\mathrm{Na}(1)-\mathrm{O}(61)$ & $79.7(5)$ & $\mathrm{O}(3)-\mathrm{Na}(1)-\mathrm{O}(61)$ & $72.4(4)$ \\
\hline $\mathrm{O}(43) \# 1-\mathrm{Na}(1)-\mathrm{O}(61)$ & $140.1(6)$ & $\mathrm{O}(39 \mathrm{~W})-\mathrm{Na}(1)-\mathrm{O}(61)$ & $64.8(5)$ \\
\hline $\mathrm{O}(33 \mathrm{~W})-\mathrm{Na}(1)-\mathrm{Na}(7)$ & $98.6(6)$ & $\mathrm{O}(5 \mathrm{~W})-\mathrm{Na}(1)-\mathrm{Na}(7)$ & $124.6(6)$ \\
\hline $\mathrm{O}(12 \mathrm{~W})-\mathrm{Na}(1)-\mathrm{Na}(7)$ & $46.4(4)$ & $\mathrm{O}(3)-\mathrm{Na}(1)-\mathrm{Na}(7)$ & $103.1(5)$ \\
\hline
\end{tabular}




\begin{tabular}{|c|c|c|c|}
\hline $\mathrm{O}(43) \# 1-\mathrm{Na}(1)-\mathrm{Na}(7)$ & $153.1(6)$ & $\mathrm{O}(39 \mathrm{~W})-\mathrm{Na}(1)-\mathrm{Na}(7)$ & $50.7(5)$ \\
\hline $\mathrm{O}(61)-\mathrm{Na}(1)-\mathrm{Na}(7)$ & $48.6(4)$ & $\mathrm{O}(33 \mathrm{~W})-\mathrm{Na}(1)-\mathrm{K}(2)$ & $53.4(6)$ \\
\hline $\mathrm{O}(5 \mathrm{~W})-\mathrm{Na}(1)-\mathrm{K}(2)$ & $44.7(4)$ & $\mathrm{O}(12 \mathrm{~W})-\mathrm{Na}(1)-\mathrm{K}(2)$ & $142.6(5)$ \\
\hline $\mathrm{O}(3)-\mathrm{Na}(1)-\mathrm{K}(2)$ & $133.0(6)$ & $\mathrm{O}(43) \# 1-\mathrm{Na}(1)-\mathrm{K}(2)$ & $90.9(5)$ \\
\hline $\mathrm{O}(39 \mathrm{~W})-\mathrm{Na}(1)-\mathrm{K}(2)$ & $60.7(5)$ & $\mathrm{O}(61)-\mathrm{Na}(1)-\mathrm{K}(2)$ & $107.7(5)$ \\
\hline $\mathrm{Na}(7)-\mathrm{Na}(1)-\mathrm{K}(2)$ & $111.2(5)$ & $\mathrm{O}(33 \mathrm{~W})-\mathrm{Na}(1)-\mathrm{U}(1)$ & $151.6(6)$ \\
\hline $\mathrm{O}(5 \mathrm{~W})-\mathrm{Na}(1)-\mathrm{U}(1)$ & $73.6(4)$ & $\mathrm{O}(12 \mathrm{~W})-\mathrm{Na}(1)-\mathrm{U}(1)$ & $99.1(4)$ \\
\hline $\mathrm{O}(3)-\mathrm{Na}(1)-\mathrm{U}(1)$ & $16.0(2)$ & $\mathrm{O}(43) \# 1-\mathrm{Na}(1)-\mathrm{U}(1)$ & $70.7(3)$ \\
\hline $\mathrm{O}(39 \mathrm{~W})-\mathrm{Na}(1)-\mathrm{U}(1)$ & $129.5(5)$ & $\mathrm{O}(61)-\mathrm{Na}(1)-\mathrm{U}(1)$ & $69.4(3)$ \\
\hline $\mathrm{Na}(7)-\mathrm{Na}(1)-\mathrm{U}(1)$ & $109.2(4)$ & $\mathrm{K}(2)-\mathrm{Na}(1)-\mathrm{U}(1)$ & $117.9(4)$ \\
\hline $\mathrm{O}(33 \mathrm{~W})-\mathrm{Na}(1)-\mathrm{U}(3) \# 1$ & $72.1(4)$ & $\mathrm{O}(5 \mathrm{~W})-\mathrm{Na}(1)-\mathrm{U}(3) \# 1$ & $74.4(4)$ \\
\hline $\mathrm{O}(12 \mathrm{~W})-\mathrm{Na}(1)-\mathrm{U}(3) \# 1$ & $116.0(5)$ & $\mathrm{O}(3)-\mathrm{Na}(1)-\mathrm{U}(3) \# 1$ & $80.6(4)$ \\
\hline $\mathrm{O}(43) \# 1-\mathrm{Na}(1)-\mathrm{U}(3) \# 1$ & $13.3(2)$ & $\mathrm{O}(39 \mathrm{~W})-\mathrm{Na}(1)-\mathrm{U}(3) \# 1$ & $136.6(6)$ \\
\hline $\mathrm{O}(61)-\mathrm{Na}(1)-\mathrm{U}(3) \# 1$ & $147.4(5)$ & $\mathrm{Na}(7)-\mathrm{Na}(1)-\mathrm{U}(3) \# 1$ & $160.3(5)$ \\
\hline $\mathrm{K}(2)-\mathrm{Na}(1)-\mathrm{U}(3) \# 1$ & $77.8(3)$ & $\mathrm{U}(1)-\mathrm{Na}(1)-\mathrm{U}(3) \# 1$ & $79.6(3)$ \\
\hline $\mathrm{O}(10 \mathrm{~W})-\mathrm{Na}(2)-\mathrm{O}(36 \mathrm{~W})$ & $91.0(5)$ & $\mathrm{O}(10 \mathrm{~W})-\mathrm{Na}(2)-\mathrm{O}(25 \mathrm{~W})$ & $86.5(5)$ \\
\hline $\mathrm{O}(36 \mathrm{~W})-\mathrm{Na}(2)-\mathrm{O}(25 \mathrm{~W})$ & $98.3(5)$ & $\mathrm{O}(10 \mathrm{~W})-\mathrm{Na}(2)-\mathrm{O}(14 \mathrm{~W})$ & $174.0(7)$ \\
\hline $\mathrm{O}(36 \mathrm{~W})-\mathrm{Na}(2)-\mathrm{O}(14 \mathrm{~W})$ & $83.1(5)$ & $\mathrm{O}(25 \mathrm{~W})-\mathrm{Na}(2)-\mathrm{O}(14 \mathrm{~W})$ & $93.5(6)$ \\
\hline $\mathrm{O}(10 \mathrm{~W})-\mathrm{Na}(2)-\mathrm{O}(14)$ & $97.8(6)$ & $\mathrm{O}(36 \mathrm{~W})-\mathrm{Na}(2)-\mathrm{O}(14)$ & $92.9(6)$ \\
\hline $\mathrm{O}(25 \mathrm{~W})-\mathrm{Na}(2)-\mathrm{O}(14)$ & $168.0(7)$ & $\mathrm{O}(14 \mathrm{~W})-\mathrm{Na}(2)-\mathrm{O}(14)$ & $83.4(5)$ \\
\hline $\mathrm{O}(10 \mathrm{~W})-\mathrm{Na}(2)-\mathrm{O}(1 \mathrm{~W}) \# 4$ & $102.8(6)$ & $\mathrm{O}(36 \mathrm{~W})-\mathrm{Na}(2)-\mathrm{O}(1 \mathrm{~W}) \# 4$ & $166.2(7)$ \\
\hline $\mathrm{O}(25 \mathrm{~W})-\mathrm{Na}(2)-\mathrm{O}(1 \mathrm{~W}) \# 4$ & $82.9(6)$ & $\mathrm{O}(14 \mathrm{~W})-\mathrm{Na}(2)-\mathrm{O}(1 \mathrm{~W}) \# 4$ & $83.1(5)$ \\
\hline $\mathrm{O}(14)-\mathrm{Na}(2)-\mathrm{O}(1 \mathrm{~W}) \# 4$ & $85.2(5)$ & $\mathrm{O}(10 \mathrm{~W})-\mathrm{Na}(2)-\mathrm{Na}(9)$ & $132.5(6)$ \\
\hline $\mathrm{O}(36 \mathrm{~W})-\mathrm{Na}(2)-\mathrm{Na}(9)$ & $41.5(4)$ & $\mathrm{O}(25 \mathrm{~W})-\mathrm{Na}(2)-\mathrm{Na}(9)$ & $98.2(5)$ \\
\hline $\mathrm{O}(14 \mathrm{~W})-\mathrm{Na}(2)-\mathrm{Na}(9)$ & $41.6(4)$ & $\mathrm{O}(14)-\mathrm{Na}(2)-\mathrm{Na}(9)$ & $87.2(5)$ \\
\hline $\mathrm{O}(1 \mathrm{~W}) \# 4-\mathrm{Na}(2)-\mathrm{Na}(9)$ & $124.7(5)$ & $\mathrm{O}(10 \mathrm{~W})-\mathrm{Na}(2)-\mathrm{Na}(12) \# 4$ & $125.8(6)$ \\
\hline $\mathrm{O}(36 \mathrm{~W})-\mathrm{Na}(2)-\mathrm{Na}(12) \# 4$ & $134.3(5)$ & $\mathrm{O}(25 \mathrm{~W})-\mathrm{Na}(2)-\mathrm{Na}(12) \# 4$ & $62.9(4)$ \\
\hline $\mathrm{O}(14 \mathrm{~W})-\mathrm{Na}(2)-\mathrm{Na}(12) \# 4$ & $59.0(4)$ & $\mathrm{O}(14)-\mathrm{Na}(2)-\mathrm{Na}(12) \# 4$ & $106.0(5)$ \\
\hline $\mathrm{O}(1 \mathrm{~W}) \# 4-\mathrm{Na}(2)-\mathrm{Na}(12) \# 4$ & $35.0(4)$ & $\mathrm{Na}(9)-\mathrm{Na}(2)-\mathrm{Na}(12) \# 4$ & $97.2(4)$ \\
\hline $\mathrm{O}(10 \mathrm{~W})-\mathrm{Na}(2)-\mathrm{U}(2)$ & $107.6(5)$ & $\mathrm{O}(36 \mathrm{~W})-\mathrm{Na}(2)-\mathrm{U}(2)$ & $101.6(5)$ \\
\hline $\mathrm{O}(25 \mathrm{~W})-\mathrm{Na}(2)-\mathrm{U}(2)$ & $155.3(5)$ & $\mathrm{O}(14 \mathrm{~W})-\mathrm{Na}(2)-\mathrm{U}(2)$ & $74.6(4)$ \\
\hline $\mathrm{O}(14)-\mathrm{Na}(2)-\mathrm{U}(2)$ & $13.5(2)$ & $\mathrm{O}(1 \mathrm{~W}) \# 4-\mathrm{Na}(2)-\mathrm{U}(2)$ & $74.2(4)$ \\
\hline $\mathrm{Na}(9)-\mathrm{Na}(2)-\mathrm{U}(2)$ & $87.3(4)$ & $\mathrm{Na}(12) \# 4-\mathrm{Na}(2)-\mathrm{U}(2)$ & $92.6(3)$ \\
\hline $\mathrm{O}(62 \mathrm{~W})-\mathrm{Na}(3)-\mathrm{O}(46)$ & $90.9(7)$ & $\mathrm{O}(62 \mathrm{~W})-\mathrm{Na}(3)-\mathrm{O}(3 \mathrm{I})$ & $85.2(7)$ \\
\hline $\mathrm{O}(46)-\mathrm{Na}(3)-\mathrm{O}(3 \mathrm{I})$ & $133.6(7)$ & $\mathrm{O}(62 \mathrm{~W})-\mathrm{Na}(3)-\mathrm{O}(22)$ & $85.0(6)$ \\
\hline $\mathrm{O}(46)-\mathrm{Na}(3)-\mathrm{O}(22)$ & $84.3(5)$ & $\mathrm{O}(3 \mathrm{I})-\mathrm{Na}(3)-\mathrm{O}(22)$ & $140.9(6)$ \\
\hline $\mathrm{O}(62 \mathrm{~W})-\mathrm{Na}(3)-\mathrm{O}(45)$ & $134.6(7)$ & $\mathrm{O}(46)-\mathrm{Na}(3)-\mathrm{O}(45)$ & $129.7(7)$ \\
\hline $\mathrm{O}(3 \mathrm{I})-\mathrm{Na}(3)-\mathrm{O}(45)$ & $79.7(5)$ & $\mathrm{O}(22)-\mathrm{Na}(3)-\mathrm{O}(45)$ & $80.7(5)$ \\
\hline $\mathrm{O}(62 \mathrm{~W})-\mathrm{Na}(3)-\mathrm{O}(38)$ & $141.3(8)$ & $\mathrm{O}(46)-\mathrm{Na}(3)-\mathrm{O}(38)$ & $77.1(4)$ \\
\hline $\mathrm{O}(3 \mathrm{I})-\mathrm{Na}(3)-\mathrm{O}(38)$ & $77.7(5)$ & $\mathrm{O}(22)-\mathrm{Na}(3)-\mathrm{O}(38)$ & $129.0(7)$ \\
\hline $\mathrm{O}(45)-\mathrm{Na}(3)-\mathrm{O}(38)$ & $76.1(5)$ & $\mathrm{O}(62 \mathrm{~W})-\mathrm{Na}(3)-\mathrm{I}(1)$ & $114.4(7)$ \\
\hline $\mathrm{O}(46)-\mathrm{Na}(3)-\mathrm{I}(1)$ & $130.0(5)$ & $\mathrm{O}(3 \mathrm{I})-\mathrm{Na}(3)-\mathrm{I}(1)$ & $29.3(3)$ \\
\hline $\mathrm{O}(22)-\mathrm{Na}(3)-\mathrm{I}(1)$ & $137.2(6)$ & $\mathrm{O}(45)-\mathrm{Na}(3)-\mathrm{I}(1)$ & $58.3(4)$ \\
\hline $\mathrm{O}(38)-\mathrm{Na}(3)-\mathrm{I}(1)$ & $55.8(4)$ & $\mathrm{O}(62 \mathrm{~W})-\mathrm{Na}(3)-\mathrm{U}(5)$ & $104.4(7)$ \\
\hline
\end{tabular}




\begin{tabular}{|c|c|c|c|}
\hline $\mathrm{O}(46)-\mathrm{Na}(3)-\mathrm{U}(5)$ & $16.7(3)$ & $\mathrm{O}(3 \mathrm{I})-\mathrm{Na}(3)-\mathrm{U}(5)$ & $142.8(5)$ \\
\hline $\mathrm{O}(22)-\mathrm{Na}(3)-\mathrm{U}(5)$ & $76.2(4)$ & $\mathrm{O}(45)-\mathrm{Na}(3)-\mathrm{U}(5)$ & $113.4(5)$ \\
\hline $\mathrm{O}(38)-\mathrm{Na}(3)-\mathrm{U}(5)$ & $72.7(3)$ & $\mathrm{I}(1)-\mathrm{Na}(3)-\mathrm{U}(5)$ & $128.4(4)$ \\
\hline $\mathrm{O}(62 \mathrm{~W})-\mathrm{Na}(3)-\mathrm{U}(3)$ & $100.7(6)$ & $\mathrm{O}(46)-\mathrm{Na}(3)-\mathrm{U}(3)$ & $75.9(4)$ \\
\hline $\mathrm{O}(3 \mathrm{I})-\mathrm{Na}(3)-\mathrm{U}(3)$ & $150.2(6)$ & $\mathrm{O}(22)-\mathrm{Na}(3)-\mathrm{U}(3)$ & $17.8(2)$ \\
\hline $\mathrm{O}(45)-\mathrm{Na}(3)-\mathrm{U}(3)$ & $75.4(4)$ & $\mathrm{O}(38)-\mathrm{Na}(3)-\mathrm{U}(3)$ & $111.4(5)$ \\
\hline $\mathrm{I}(1)-\mathrm{Na}(3)-\mathrm{U}(3)$ & $133.5(5)$ & $U(5)-N a(3)-U(3)$ & $64.3(2)$ \\
\hline $\mathrm{O}(62 \mathrm{~W})-\mathrm{Na}(3)-\mathrm{U}(4)$ & $145.5(7)$ & $\mathrm{O}(46)-\mathrm{Na}(3)-\mathrm{U}(4)$ & $112.6(5)$ \\
\hline $\mathrm{O}(3 \mathrm{I})-\mathrm{Na}(3)-\mathrm{U}(4)$ & $94.9(5)$ & $\mathrm{O}(22)-\mathrm{Na}(3)-\mathrm{U}(4)$ & $73.3(4)$ \\
\hline $\mathrm{O}(45)-\mathrm{Na}(3)-\mathrm{U}(4)$ & $17.4(2)$ & $\mathrm{O}(38)-\mathrm{Na}(3)-\mathrm{U}(4)$ & $71.1(4)$ \\
\hline $\mathrm{I}(1)-\mathrm{Na}(3)-\mathrm{U}(4)$ & $70.0(3)$ & $U(5)-N a(3)-U(4)$ & $96.1(3)$ \\
\hline $\mathrm{U}(3)-\mathrm{Na}(3)-\mathrm{U}(4)$ & $63.8(3)$ & $\mathrm{O}(62 \mathrm{~W})-\mathrm{Na}(3)-\mathrm{U}(7)$ & $152.0(7)$ \\
\hline $\mathrm{O}(46)-\mathrm{Na}(3)-\mathrm{U}(7)$ & $70.8(4)$ & $\mathrm{O}(3 \mathrm{I})-\mathrm{Na}(3)-\mathrm{U}(7)$ & $92.3(4)$ \\
\hline $\mathrm{O}(22)-\mathrm{Na}(3)-\mathrm{U}(7)$ & $112.9(5)$ & $\mathrm{O}(45)-\mathrm{Na}(3)-\mathrm{U}(7)$ & $71.7(4)$ \\
\hline $\mathrm{O}(38)-\mathrm{Na}(3)-\mathrm{U}(7)$ & $16.2(2)$ & $\mathrm{I}(1)-\mathrm{Na}(3)-\mathrm{U}(7)$ & $67.5(3)$ \\
\hline$U(5)-N a(3)-U(7)$ & $62.4(2)$ & $U(3)-N a(3)-U(7)$ & $95.2(3)$ \\
\hline $\mathrm{U}(4)-\mathrm{Na}(3)-\mathrm{U}(7)$ & $62.4(2)$ & $\mathrm{O}(77)-\mathrm{Na}(4)-\mathrm{O}(20 \mathrm{~W})$ & $165.7(7)$ \\
\hline $\mathrm{O}(77)-\mathrm{Na}(4)-\mathrm{O}(27 \mathrm{~W})$ & $95.6(6)$ & $\mathrm{O}(20 \mathrm{~W})-\mathrm{Na}(4)-\mathrm{O}(27 \mathrm{~W})$ & $93.0(6)$ \\
\hline $\mathrm{O}(77)-\mathrm{Na}(4)-\mathrm{O}(18 \mathrm{~W})$ & $106.0(5)$ & $\mathrm{O}(20 \mathrm{~W})-\mathrm{Na}(4)-\mathrm{O}(18 \mathrm{~W})$ & $84.5(5)$ \\
\hline $\mathrm{O}(27 \mathrm{~W})-\mathrm{Na}(4)-\mathrm{O}(18 \mathrm{~W})$ & $95.1(6)$ & $\mathrm{O}(77)-\mathrm{Na}(4)-\mathrm{O}(16 \mathrm{~W}) \# 5$ & $88.0(5)$ \\
\hline $\mathrm{O}(20 \mathrm{~W})-\mathrm{Na}(4)-\mathrm{O}(16 \mathrm{~W}) \# 5$ & $82.3(5)$ & $\mathrm{O}(27 \mathrm{~W})-\mathrm{Na}(4)-\mathrm{O}(16 \mathrm{~W}) \# 5$ & $173.0(7)$ \\
\hline $\mathrm{O}(18 \mathrm{~W})-\mathrm{Na}(4)-\mathrm{O}(16 \mathrm{~W}) \# 5$ & $89.7(5)$ & $\mathrm{O}(77)-\mathrm{Na}(4)-\mathrm{O}(77) \# 5$ & $87.3(5)$ \\
\hline $\mathrm{O}(20 \mathrm{~W})-\mathrm{Na}(4)-\mathrm{O}(77) \# 5$ & $82.4(5)$ & $\mathrm{O}(27 \mathrm{~W})-\mathrm{Na}(4)-\mathrm{O}(77) \# 5$ & $83.3(5)$ \\
\hline $\mathrm{O}(18 \mathrm{~W})-\mathrm{Na}(4)-\mathrm{O}(77) \# 5$ & $166.7(6)$ & $\mathrm{O}(16 \mathrm{~W}) \# 5-\mathrm{Na}(4)-\mathrm{O}(77) \# 5$ & $90.9(5)$ \\
\hline $\mathrm{O}(77)-\mathrm{Na}(4)-\mathrm{Na}(14) \# 5$ & $93.0(5)$ & $\mathrm{O}(20 \mathrm{~W})-\mathrm{Na}(4)-\mathrm{Na}(14) \# 5$ & $72.7(5)$ \\
\hline $\mathrm{O}(27 \mathrm{~W})-\mathrm{Na}(4)-\mathrm{Na}(14) \# 5$ & $128.4(6)$ & $\mathrm{O}(18 \mathrm{~W})-\mathrm{Na}(4)-\mathrm{Na}(14) \# 5$ & $130.7(6)$ \\
\hline $\mathrm{O}(16 \mathrm{~W}) \# 5-\mathrm{Na}(4)-\mathrm{Na}(14) \# 5$ & $45.2(4)$ & $\mathrm{O}(77) \# 5-\mathrm{Na}(4)-\mathrm{Na}(14) \# 5$ & $46.4(4)$ \\
\hline $\mathrm{O}(77)-\mathrm{Na}(4)-\mathrm{Na}(4) \# 5$ & $44.7(3)$ & $\mathrm{O}(20 \mathrm{~W})-\mathrm{Na}(4)-\mathrm{Na}(4) \mathrm{\# 5}$ & $124.3(7)$ \\
\hline $\mathrm{O}(27 \mathrm{~W})-\mathrm{Na}(4)-\mathrm{Na}(4) \# 5$ & $89.1(6)$ & $\mathrm{O}(18 \mathrm{~W})-\mathrm{Na}(4)-\mathrm{Na}(4) \mathrm{\# 5}$ & $150.7(6)$ \\
\hline $\mathrm{O}(16 \mathrm{~W}) \# 5-\mathrm{Na}(4)-\mathrm{Na}(4) \# 5$ & $89.2(6)$ & $\mathrm{O}(77) \# 5-\mathrm{Na}(4)-\mathrm{Na}(4) \# 5$ & $42.6(3)$ \\
\hline $\mathrm{Na}(14) \# 5-\mathrm{Na}(4)-\mathrm{Na}(4) \# 5$ & $63.3(5)$ & $\mathrm{O}(77)-\mathrm{Na}(4)-\mathrm{Na}(19) \# 5$ & $143.2(6)$ \\
\hline $\mathrm{O}(20 \mathrm{~W})-\mathrm{Na}(4)-\mathrm{Na}(19) \# 5$ & $43.1(5)$ & $\mathrm{O}(27 \mathrm{~W})-\mathrm{Na}(4)-\mathrm{Na}(19) \# 5$ & $50.0(6)$ \\
\hline $\mathrm{O}(18 \mathrm{~W})-\mathrm{Na}(4)-\mathrm{Na}(19) \# 5$ & $91.6(6)$ & $\mathrm{O}(16 \mathrm{~W}) \# 5-\mathrm{Na}(4)-\mathrm{Na}(19) \# 5$ & $124.9(6)$ \\
\hline $\mathrm{O}(77) \# 5-\mathrm{Na}(4)-\mathrm{Na}(19) \# 5$ & $77.2(5)$ & $\mathrm{Na}(14) \# 5-\mathrm{Na}(4)-\mathrm{Na}(19) \# 5$ & $99.5(6)$ \\
\hline $\mathrm{Na}(4) \# 5-\mathrm{Na}(4)-\mathrm{Na}(19) \# 5$ & $112.8(6)$ & $\mathrm{O}(77)-\mathrm{Na}(4)-\mathrm{Na}(14)$ & $42.5(4)$ \\
\hline $\mathrm{O}(20 \mathrm{~W})-\mathrm{Na}(4)-\mathrm{Na}(14)$ & $145.6(6)$ & $\mathrm{O}(27 \mathrm{~W})-\mathrm{Na}(4)-\mathrm{Na}(14)$ & $53.3(5)$ \\
\hline $\mathrm{O}(18 \mathrm{~W})-\mathrm{Na}(4)-\mathrm{Na}(14)$ & $103.4(5)$ & $\mathrm{O}(16 \mathrm{~W}) \# 5-\mathrm{Na}(4)-\mathrm{Na}(14)$ & $130.4(5)$ \\
\hline $\mathrm{O}(77) \# 5-\mathrm{Na}(4)-\mathrm{Na}(14)$ & $86.3(5)$ & $\mathrm{Na}(14) \# 5-\mathrm{Na}(4)-\mathrm{Na}(14)$ & $120.2(4)$ \\
\hline $\mathrm{Na}(4) \# 5-\mathrm{Na}(4)-\mathrm{Na}(14)$ & $56.9(5)$ & $\mathrm{Na}(19) \# 5-\mathrm{Na}(4)-\mathrm{Na}(14)$ & $102.6(5)$ \\
\hline $\mathrm{O}(2 \mathrm{l})-\mathrm{Na}(5)-\mathrm{O}(9 \mathrm{~W}) \# 2$ & $106.1(6)$ & $\mathrm{O}(2 \mathrm{I})-\mathrm{Na}(5)-\mathrm{O}(34 \mathrm{~W})$ & $80.8(6)$ \\
\hline $\mathrm{O}(9 \mathrm{~W}) \# 2-\mathrm{Na}(5)-\mathrm{O}(34 \mathrm{~W})$ & $84.7(5)$ & $\mathrm{O}(2 \mathrm{I})-\mathrm{Na}(5)-\mathrm{O}(43 \mathrm{~W})$ & $84.1(6)$ \\
\hline $\mathrm{O}(9 \mathrm{~W}) \# 2-\mathrm{Na}(5)-\mathrm{O}(43 \mathrm{~W})$ & $169.5(8)$ & $\mathrm{O}(34 \mathrm{~W})-\mathrm{Na}(5)-\mathrm{O}(43 \mathrm{~W})$ & $94.4(6)$ \\
\hline $\mathrm{O}(2 \mathrm{I})-\mathrm{Na}(5)-\mathrm{O}(76)$ & $113.7(7)$ & $\mathrm{O}(9 \mathrm{~W}) \# 2-\mathrm{Na}(5)-\mathrm{O}(76)$ & $90.8(5)$ \\
\hline $\mathrm{O}(34 \mathrm{~W})-\mathrm{Na}(5)-\mathrm{O}(76)$ & $165.4(8)$ & $\mathrm{O}(43 \mathrm{~W})-\mathrm{Na}(5)-\mathrm{O}(76)$ & $87.4(6)$ \\
\hline
\end{tabular}




\begin{tabular}{|c|c|c|c|}
\hline $\mathrm{O}(2 \mathrm{I})-\mathrm{Na}(5)-\mathrm{O}(15 \mathrm{~W})$ & $156.4(7)$ & $\mathrm{O}(9 \mathrm{~W}) \# 2-\mathrm{Na}(5)-\mathrm{O}(15 \mathrm{~W})$ & $84.1(5)$ \\
\hline $\mathrm{O}(34 \mathrm{~W})-\mathrm{Na}(5)-\mathrm{O}(15 \mathrm{~W})$ & $78.9(5)$ & $\mathrm{O}(43 \mathrm{~W})-\mathrm{Na}(5)-\mathrm{O}(15 \mathrm{~W})$ & $85.5(6)$ \\
\hline $\mathrm{O}(76)-\mathrm{Na}(5)-\mathrm{O}(15 \mathrm{~W})$ & $86.8(5)$ & $\mathrm{O}(2 \mathrm{I})-\mathrm{Na}(5)-\mathrm{Na}(6) \# 2$ & $113.2(6)$ \\
\hline $\mathrm{O}(9 \mathrm{~W}) \# 2-\mathrm{Na}(5)-\mathrm{Na}(6) \# 2$ & $44.4(4)$ & $\mathrm{O}(34 \mathrm{~W})-\mathrm{Na}(5)-\mathrm{Na}(6) \# 2$ & $128.9(6)$ \\
\hline $\mathrm{O}(43 \mathrm{~W})-\mathrm{Na}(5)-\mathrm{Na}(6) \# 2$ & $134.3(7)$ & $\mathrm{O}(76)-\mathrm{Na}(5)-\mathrm{Na}(6) \# 2$ & $47.0(4)$ \\
\hline $\mathrm{O}(15 \mathrm{~W})-\mathrm{Na}(5)-\mathrm{Na}(6) \# 2$ & $89.1(5)$ & $\mathrm{O}(2 \mathrm{I})-\mathrm{Na}(5)-\mathrm{Na}(6)$ & $154.5(6)$ \\
\hline $\mathrm{O}(9 \mathrm{~W}) \# 2-\mathrm{Na}(5)-\mathrm{Na}(6)$ & $88.8(5)$ & $\mathrm{O}(34 \mathrm{~W})-\mathrm{Na}(5)-\mathrm{Na}(6)$ & $121.9(6)$ \\
\hline $\mathrm{O}(43 \mathrm{~W})-\mathrm{Na}(5)-\mathrm{Na}(6)$ & $82.8(6)$ & $\mathrm{O}(76)-\mathrm{Na}(5)-\mathrm{Na}(6)$ & $43.9(4)$ \\
\hline $\mathrm{O}(15 \mathrm{~W})-\mathrm{Na}(5)-\mathrm{Na}(6)$ & $43.0(4)$ & $\mathrm{Na}(6) \# 2-\mathrm{Na}(5)-\mathrm{Na}(6)$ & $63.5(4)$ \\
\hline $\mathrm{O}(2 \mathrm{I})-\mathrm{Na}(5)-\mathrm{Na}(20)$ & $44.3(6)$ & $\mathrm{O}(9 \mathrm{~W}) \# 2-\mathrm{Na}(5)-\mathrm{Na}(20)$ & $143.7(8)$ \\
\hline $\mathrm{O}(34 \mathrm{~W})-\mathrm{Na}(5)-\mathrm{Na}(20)$ & $71.8(6)$ & $\mathrm{O}(43 \mathrm{~W})-\mathrm{Na}(5)-\mathrm{Na}(20)$ & $44.2(6)$ \\
\hline $\mathrm{O}(76)-\mathrm{Na}(5)-\mathrm{Na}(20)$ & $118.2(7)$ & $\mathrm{O}(15 \mathrm{~W})-\mathrm{Na}(5)-\mathrm{Na}(20)$ & $116.6(7)$ \\
\hline $\mathrm{Na}(6) \# 2-\mathrm{Na}(5)-\mathrm{Na}(20)$ & $151.4(7)$ & $\mathrm{Na}(6)-\mathrm{Na}(5)-\mathrm{Na}(20)$ & $127.0(6)$ \\
\hline $\mathrm{O}(2 \mathrm{I})-\mathrm{Na}(5)-\mathrm{Na}(21)$ & $40.2(10)$ & $\mathrm{O}(9 \mathrm{~W}) \# 2-\mathrm{Na}(5)-\mathrm{Na}(21)$ & $127.0(11)$ \\
\hline $\mathrm{O}(34 \mathrm{~W})-\mathrm{Na}(5)-\mathrm{Na}(21)$ & $56.4(10)$ & $\mathrm{O}(43 \mathrm{~W})-\mathrm{Na}(5)-\mathrm{Na}(21)$ & $59.5(10)$ \\
\hline $\mathrm{O}(76)-\mathrm{Na}(5)-\mathrm{Na}(21)$ & $135.1(11)$ & $\mathrm{O}(15 \mathrm{~W})-\mathrm{Na}(5)-\mathrm{Na}(21)$ & $116.8(11)$ \\
\hline $\mathrm{Na}(6) \# 2-\mathrm{Na}(5)-\mathrm{Na}(21)$ & $153.2(11)$ & $\mathrm{Na}(6)-\mathrm{Na}(5)-\mathrm{Na}(21)$ & $140.6(10)$ \\
\hline $\mathrm{Na}(20)-\mathrm{Na}(5)-\mathrm{Na}(21)$ & $17.9(11)$ & $\mathrm{O}(9 \mathrm{~W})-\mathrm{Na}(6)-\mathrm{O}(15 \mathrm{~W})$ & $172.0(7)$ \\
\hline $\mathrm{O}(9 \mathrm{~W})-\mathrm{Na}(6)-\mathrm{O}(67 \mathrm{~W})$ & $92.6(6)$ & $\mathrm{O}(15 \mathrm{~W})-\mathrm{Na}(6)-\mathrm{O}(67 \mathrm{~W})$ & $94.9(6)$ \\
\hline $\mathrm{O}(9 \mathrm{~W})-\mathrm{Na}(6)-\mathrm{O}(76)$ & $83.8(5)$ & $\mathrm{O}(15 \mathrm{~W})-\mathrm{Na}(6)-\mathrm{O}(76)$ & $91.2(6)$ \\
\hline $\mathrm{O}(67 \mathrm{~W})-\mathrm{Na}(6)-\mathrm{O}(76)$ & $108.5(6)$ & $\mathrm{O}(9 \mathrm{~W})-\mathrm{Na}(6)-\mathrm{O}(46 \mathrm{~W})$ & $77.5(5)$ \\
\hline $\mathrm{O}(15 \mathrm{~W})-\mathrm{Na}(6)-\mathrm{O}(46 \mathrm{~W})$ & $105.4(6)$ & $\mathrm{O}(67 \mathrm{~W})-\mathrm{Na}(6)-\mathrm{O}(46 \mathrm{~W})$ & $89.1(6)$ \\
\hline $\mathrm{O}(76)-\mathrm{Na}(6)-\mathrm{O}(46 \mathrm{~W})$ & $154.9(7)$ & $\mathrm{O}(9 \mathrm{~W})-\mathrm{Na}(6)-\mathrm{O}(76) \# 2$ & $89.4(5)$ \\
\hline $\mathrm{O}(15 \mathrm{~W})-\mathrm{Na}(6)-\mathrm{O}(76) \# 2$ & $83.9(5)$ & $\mathrm{O}(67 \mathrm{~W})-\mathrm{Na}(6)-\mathrm{O}(76) \# 2$ & $166.7(7)$ \\
\hline $\mathrm{O}(76)-\mathrm{Na}(6)-\mathrm{O}(76) \# 2$ & $84.8(5)$ & $\mathrm{O}(46 \mathrm{~W})-\mathrm{Na}(6)-\mathrm{O}(76) \# 2$ & $78.5(5)$ \\
\hline $\mathrm{O}(9 \mathrm{~W})-\mathrm{Na}(6)-\mathrm{Na}(5) \# 2$ & $44.1(4)$ & $\mathrm{O}(15 \mathrm{~W})-\mathrm{Na}(6)-\mathrm{Na}(5) \# 2$ & $129.7(6)$ \\
\hline $\mathrm{O}(67 \mathrm{~W})-\mathrm{Na}(6)-\mathrm{Na}(5) \# 2$ & $132.9(7)$ & $\mathrm{O}(76)-\mathrm{Na}(6)-\mathrm{Na}(5) \# 2$ & $87.5(5)$ \\
\hline $\mathrm{O}(46 \mathrm{~W})-\mathrm{Na}(6)-\mathrm{Na}(5) \# 2$ & $67.4(5)$ & $\mathrm{O}(76) \# 2-\mathrm{Na}(6)-\mathrm{Na}(5) \# 2$ & $45.8(4)$ \\
\hline $\mathrm{O}(9 \mathrm{~W})-\mathrm{Na}(6)-\mathrm{Na}(5)$ & $128.1(6)$ & $\mathrm{O}(15 \mathrm{~W})-\mathrm{Na}(6)-\mathrm{Na}(5)$ & $47.0(4)$ \\
\hline $\mathrm{O}(67 \mathrm{~W})-\mathrm{Na}(6)-\mathrm{Na}(5)$ & $104.5(6)$ & $\mathrm{O}(76)-\mathrm{Na}(6)-\mathrm{Na}(5)$ & $44.3(4)$ \\
\hline $\mathrm{O}(46 \mathrm{~W})-\mathrm{Na}(6)-\mathrm{Na}(5)$ & $149.3(6)$ & $\mathrm{O}(76) \# 2-\mathrm{Na}(6)-\mathrm{Na}(5)$ & $84.4(5)$ \\
\hline $\mathrm{Na}(5) \# 2-\mathrm{Na}(6)-\mathrm{Na}(5)$ & $116.5(4)$ & $\mathrm{O}(9 \mathrm{~W})-\mathrm{Na}(6)-\mathrm{Na}(6) \# 2$ & $85.5(5)$ \\
\hline $\mathrm{O}(15 \mathrm{~W})-\mathrm{Na}(6)-\mathrm{Na}(6) \# 2$ & $86.6(6)$ & $\mathrm{O}(67 \mathrm{~W})-\mathrm{Na}(6)-\mathrm{Na}(6) \# 2$ & $151.6(8)$ \\
\hline $\mathrm{O}(76)-\mathrm{Na}(6)-\mathrm{Na}(6) \# 2$ & $43.1(3)$ & $\mathrm{O}(46 \mathrm{~W})-\mathrm{Na}(6)-\mathrm{Na}(6) \# 2$ & $117.9(7)$ \\
\hline $\mathrm{O}(76) \# 2-\mathrm{Na}(6)-\mathrm{Na}(6) \# 2$ & $41.7(3)$ & $\mathrm{Na}(5) \# 2-\mathrm{Na}(6)-\mathrm{Na}(6) \# 2$ & $59.5(4)$ \\
\hline $\mathrm{Na}(5)-\mathrm{Na}(6)-\mathrm{Na}(6) \# 2$ & $57.0(4)$ & $\mathrm{O}(4 \mathrm{~W})-\mathrm{Na}(7)-\mathrm{O}(12 \mathrm{~W})$ & $171.4(7)$ \\
\hline $\mathrm{O}(4 \mathrm{~W})-\mathrm{Na}(7)-\mathrm{O}(76 \mathrm{~W})$ & $100.2(6)$ & $\mathrm{O}(12 \mathrm{~W})-\mathrm{Na}(7)-\mathrm{O}(76 \mathrm{~W})$ & $88.4(6)$ \\
\hline $\mathrm{O}(4 \mathrm{~W})-\mathrm{Na}(7)-\mathrm{O}(66)$ & $92.8(5)$ & $\mathrm{O}(12 \mathrm{~W})-\mathrm{Na}(7)-\mathrm{O}(66)$ & $79.5(5)$ \\
\hline $\mathrm{O}(76 \mathrm{~W})-\mathrm{Na}(7)-\mathrm{O}(66)$ & $148.2(8)$ & $\mathrm{O}(4 \mathrm{~W})-\mathrm{Na}(7)-\mathrm{O}(36)$ & $85.2(5)$ \\
\hline $\mathrm{O}(12 \mathrm{~W})-\mathrm{Na}(7)-\mathrm{O}(36)$ & $95.3(6)$ & $\mathrm{O}(76 \mathrm{~W})-\mathrm{Na}(7)-\mathrm{O}(36)$ & $84.4(5)$ \\
\hline $\mathrm{O}(66)-\mathrm{Na}(7)-\mathrm{O}(36)$ & $67.8(4)$ & $\mathrm{O}(4 \mathrm{~W})-\mathrm{Na}(7)-\mathrm{O}(61)$ & $91.8(5)$ \\
\hline $\mathrm{O}(12 \mathrm{~W})-\mathrm{Na}(7)-\mathrm{O}(61)$ & $82.0(5)$ & $\mathrm{O}(76 \mathrm{~W})-\mathrm{Na}(7)-\mathrm{O}(61)$ & $137.0(7)$ \\
\hline $\mathrm{O}(66)-\mathrm{Na}(7)-\mathrm{O}(61)$ & $70.5(4)$ & $\mathrm{O}(36)-\mathrm{Na}(7)-\mathrm{O}(61)$ & $138.0(6)$ \\
\hline $\mathrm{O}(4 \mathrm{~W})-\mathrm{Na}(7)-\mathrm{O}(39 \mathrm{~W})$ & $91.9(6)$ & $\mathrm{O}(12 \mathrm{~W})-\mathrm{Na}(7)-\mathrm{O}(39 \mathrm{~W})$ & $91.0(6)$ \\
\hline
\end{tabular}




\begin{tabular}{|c|c|c|c|}
\hline $\mathrm{O}(76 \mathrm{~W})-\mathrm{Na}(7)-\mathrm{O}(39 \mathrm{~W})$ & $73.1(5)$ & $\mathrm{O}(66)-\mathrm{Na}(7)-\mathrm{O}(39 \mathrm{~W})$ & $135.7(6)$ \\
\hline $\mathrm{O}(36)-\mathrm{Na}(7)-\mathrm{O}(39 \mathrm{~W})$ & $156.5(6)$ & $\mathrm{O}(61)-\mathrm{Na}(7)-\mathrm{O}(39 \mathrm{~W})$ & $65.3(5)$ \\
\hline $\mathrm{O}(4 \mathrm{~W})-\mathrm{Na}(7)-\mathrm{Na}(1)$ & $132.6(7)$ & $\mathrm{O}(12 \mathrm{~W})-\mathrm{Na}(7)-\mathrm{Na}(1)$ & $46.4(4)$ \\
\hline $\mathrm{O}(76 \mathrm{~W})-\mathrm{Na}(7)-\mathrm{Na}(1)$ & $92.7(5)$ & $\mathrm{O}(66)-\mathrm{Na}(7)-\mathrm{Na}(1)$ & $99.6(5)$ \\
\hline $\mathrm{O}(36)-\mathrm{Na}(7)-\mathrm{Na}(1)$ & $141.7(6)$ & $\mathrm{O}(61)-\mathrm{Na}(7)-\mathrm{Na}(1)$ & $51.5(4)$ \\
\hline $\mathrm{O}(39 \mathrm{~W})-\mathrm{Na}(7)-\mathrm{Na}(1)$ & $48.8(4)$ & $\mathrm{O}(4 \mathrm{~W})-\mathrm{Na}(7)-\mathrm{K}(1)$ & $45.6(4)$ \\
\hline $\mathrm{O}(12 \mathrm{~W})-\mathrm{Na}(7)-\mathrm{K}(1)$ & $142.7(5)$ & $\mathrm{O}(76 \mathrm{~W})-\mathrm{Na}(7)-\mathrm{K}(1)$ & $56.9(5)$ \\
\hline $\mathrm{O}(66)-\mathrm{Na}(7)-\mathrm{K}(1)$ & $137.3(5)$ & $\mathrm{O}(36)-\mathrm{Na}(7)-\mathrm{K}(1)$ & $94.6(5)$ \\
\hline $\mathrm{O}(61)-\mathrm{Na}(7)-\mathrm{K}(1)$ & $112.8(6)$ & $\mathrm{O}(39 \mathrm{~W})-\mathrm{Na}(7)-\mathrm{K}(1)$ & $67.5(5)$ \\
\hline $\mathrm{Na}(1)-\mathrm{Na}(7)-\mathrm{K}(1)$ & $115.6(5)$ & $\mathrm{O}(4 \mathrm{~W})-\mathrm{Na}(7)-\mathrm{U}(12)$ & $76.5(4)$ \\
\hline $\mathrm{O}(12 \mathrm{~W})-\mathrm{Na}(7)-\mathrm{U}(12)$ & $95.6(4)$ & $\mathrm{O}(76 \mathrm{~W})-\mathrm{Na}(7)-\mathrm{U}(12)$ & $153.7(6)$ \\
\hline $\mathrm{O}(66)-\mathrm{Na}(7)-\mathrm{U}(12)$ & $16.5(2)$ & $\mathrm{O}(36)-\mathrm{Na}(7)-\mathrm{U}(12)$ & $69.3(3)$ \\
\hline $\mathrm{O}(61)-\mathrm{Na}(7)-\mathrm{U}(12)$ & $69.2(3)$ & $\mathrm{O}(39 \mathrm{~W})-\mathrm{Na}(7)-\mathrm{U}(12)$ & $132.6(5)$ \\
\hline $\mathrm{Na}(1)-\mathrm{Na}(7)-\mathrm{U}(12)$ & $108.9(4)$ & $\mathrm{K}(1)-\mathrm{Na}(7)-\mathrm{U}(12)$ & $121.6(4)$ \\
\hline $\mathrm{O}(4 \mathrm{~W})-\mathrm{Na}(7)-\mathrm{U}(11)$ & $74.6(4)$ & $\mathrm{O}(12 \mathrm{~W})-\mathrm{Na}(7)-\mathrm{U}(11)$ & $98.8(5)$ \\
\hline $\mathrm{O}(76 \mathrm{~W})-\mathrm{Na}(7)-\mathrm{U}(11)$ & $142.9(6)$ & $\mathrm{O}(66)-\mathrm{Na}(7)-\mathrm{U}(11)$ & $68.5(3)$ \\
\hline $\mathrm{O}(36)-\mathrm{Na}(7)-\mathrm{U}(11)$ & $130.4(5)$ & $\mathrm{O}(61)-\mathrm{Na}(7)-\mathrm{U}(11)$ & $17.6(2)$ \\
\hline $\mathrm{O}(39 \mathrm{~W})-\mathrm{Na}(7)-\mathrm{U}(11)$ & $70.4(4)$ & $\mathrm{Na}(1)-\mathrm{Na}(7)-\mathrm{U}(11)$ & $68.2(3)$ \\
\hline $\mathrm{K}(1)-\mathrm{Na}(7)-\mathrm{U}(11)$ & $101.8(4)$ & $U(12)-\mathrm{Na}(7)-U(11)$ & $62.1(2)$ \\
\hline $\mathrm{O}(4 \mathrm{~W})-\mathrm{Na}(7)-\mathrm{U}(7)$ & $76.4(4)$ & $\mathrm{O}(12 \mathrm{~W})-\mathrm{Na}(7)-\mathrm{U}(7)$ & $105.9(5)$ \\
\hline $\mathrm{O}(76 \mathrm{~W})-\mathrm{Na}(7)-\mathrm{U}(7)$ & $74.3(4)$ & $\mathrm{O}(66)-\mathrm{Na}(7)-\mathrm{U}(7)$ & $81.0(4)$ \\
\hline $\mathrm{O}(36)-\mathrm{Na}(7)-\mathrm{U}(7)$ & $15.2(2)$ & $\mathrm{O}(61)-\mathrm{Na}(7)-\mathrm{U}(7)$ & $148.6(5)$ \\
\hline $\mathrm{O}(39 \mathrm{~W})-\mathrm{Na}(7)-\mathrm{U}(7)$ & $142.6(5)$ & $\mathrm{Na}(1)-\mathrm{Na}(7)-\mathrm{U}(7)$ & $150.6(5)$ \\
\hline $\mathrm{K}(1)-\mathrm{Na}(7)-\mathrm{U}(7)$ & $79.6(4)$ & $\mathrm{U}(12)-\mathrm{Na}(7)-\mathrm{U}(7)$ & $79.7(3)$ \\
\hline $\mathrm{U}(11)-\mathrm{Na}(7)-\mathrm{U}(7)$ & $136.2(3)$ & $\mathrm{O}(80) \# 6-\mathrm{Na}(8)-\mathrm{O}(24 \mathrm{~W})$ & $89.3(5)$ \\
\hline $\mathrm{O}(80) \# 6-\mathrm{Na}(8)-\mathrm{O}(80)$ & $81.9(5)$ & $\mathrm{O}(24 \mathrm{~W})-\mathrm{Na}(8)-\mathrm{O}(80)$ & $94.7(6)$ \\
\hline $\mathrm{O}(80) \# 6-\mathrm{Na}(8)-\mathrm{O}(35 \mathrm{~W})$ & $108.6(6)$ & $\mathrm{O}(24 \mathrm{~W})-\mathrm{Na}(8)-\mathrm{O}(35 \mathrm{~W})$ & $97.7(6)$ \\
\hline $\mathrm{O}(80)-\mathrm{Na}(8)-\mathrm{O}(35 \mathrm{~W})$ & $163.8(7)$ & $\mathrm{O}(80) \# 6-\mathrm{Na}(8)-\mathrm{O}(41 \mathrm{~W})$ & $94.7(6)$ \\
\hline $\mathrm{O}(24 \mathrm{~W})-\mathrm{Na}(8)-\mathrm{O}(41 \mathrm{~W})$ & $175.4(7)$ & $\mathrm{O}(80)-\mathrm{Na}(8)-\mathrm{O}(41 \mathrm{~W})$ & $88.2(6)$ \\
\hline $\mathrm{O}(35 \mathrm{~W})-\mathrm{Na}(8)-\mathrm{O}(41 \mathrm{~W})$ & $78.8(6)$ & $\mathrm{O}(80) \# 6-\mathrm{Na}(8)-\mathrm{O}(28 \mathrm{~W})$ & $168.2(7)$ \\
\hline $\mathrm{O}(24 \mathrm{~W})-\mathrm{Na}(8)-\mathrm{O}(28 \mathrm{~W})$ & $86.8(5)$ & $\mathrm{O}(80)-\mathrm{Na}(8)-\mathrm{O}(28 \mathrm{~W})$ & $87.4(5)$ \\
\hline $\mathrm{O}(35 \mathrm{~W})-\mathrm{Na}(8)-\mathrm{O}(28 \mathrm{~W})$ & $83.0(5)$ & $\mathrm{O}(41 \mathrm{~W})-\mathrm{Na}(8)-\mathrm{O}(28 \mathrm{~W})$ & $89.7(6)$ \\
\hline $\mathrm{O}(80) \# 6-\mathrm{Na}(8)-\mathrm{Na}(17) \# 6$ & $45.5(5)$ & $\mathrm{O}(24 \mathrm{~W})-\mathrm{Na}(8)-\mathrm{Na}(17) \# 6$ & $43.8(5)$ \\
\hline $\mathrm{O}(80)-\mathrm{Na}(8)-\mathrm{Na}(17) \# 6$ & $86.5(6)$ & $\mathrm{O}(35 \mathrm{~W})-\mathrm{Na}(8)-\mathrm{Na}(17) \# 6$ & 109.7(7) \\
\hline $\mathrm{O}(41 \mathrm{~W})-\mathrm{Na}(8)-\mathrm{Na}(17) \# 6$ & $140.2(6)$ & $\mathrm{O}(28 \mathrm{~W})-\mathrm{Na}(8)-\mathrm{Na}(17) \# 6$ & $129.3(7)$ \\
\hline $\mathrm{O}(80) \# 6-\mathrm{Na}(8)-\mathrm{P}(12)$ & $98.9(5)$ & $\mathrm{O}(24 \mathrm{~W})-\mathrm{Na}(8)-\mathrm{P}(12)$ & $81.3(5)$ \\
\hline $\mathrm{O}(80)-\mathrm{Na}(8)-\mathrm{P}(12)$ & $21.9(3)$ & $\mathrm{O}(35 \mathrm{~W})-\mathrm{Na}(8)-\mathrm{P}(12)$ & $152.5(6)$ \\
\hline $\mathrm{O}(41 \mathrm{~W})-\mathrm{Na}(8)-\mathrm{P}(12)$ & $100.4(5)$ & $\mathrm{O}(28 \mathrm{~W})-\mathrm{Na}(8)-\mathrm{P}(12)$ & $69.5(4)$ \\
\hline $\mathrm{Na}(17) \# 6-\mathrm{Na}(8)-\mathrm{P}(12)$ & $88.7(6)$ & $\mathrm{O}(80) \# 6-\mathrm{Na}(8)-\mathrm{P}(12) \# 6$ & $20.4(3)$ \\
\hline $\mathrm{O}(24 \mathrm{~W})-\mathrm{Na}(8)-\mathrm{P}(12) \# 6$ & $101.5(4)$ & $\mathrm{O}(80)-\mathrm{Na}(8)-\mathrm{P}(12) \# 6$ & $97.1(4)$ \\
\hline $\mathrm{O}(35 \mathrm{~W})-\mathrm{Na}(8)-\mathrm{P}(12) \# 6$ & $90.5(5)$ & $\mathrm{O}(41 \mathrm{~W})-\mathrm{Na}(8)-\mathrm{P}(12) \# 6$ & $81.7(5)$ \\
\hline $\mathrm{O}(28 \mathrm{~W})-\mathrm{Na}(8)-\mathrm{P}(12) \# 6$ & $170.1(6)$ & $\mathrm{Na}(17) \# 6-\mathrm{Na}(8)-\mathrm{P}(12) \# 6$ & $60.0(4)$ \\
\hline $\mathrm{P}(12)-\mathrm{Na}(8)-\mathrm{P}(12) \# 6$ & $116.7(4)$ & $\mathrm{O}(80) \# 6-\mathrm{Na}(8)-\mathrm{Na}(17)$ & $84.3(5)$ \\
\hline $\mathrm{O}(24 \mathrm{~W})-\mathrm{Na}(8)-\mathrm{Na}(17)$ & $138.1(7)$ & $\mathrm{O}(80)-\mathrm{Na}(8)-\mathrm{Na}(17)$ & $43.4(5)$ \\
\hline
\end{tabular}




\begin{tabular}{|c|c|c|c|}
\hline $\mathrm{O}(35 \mathrm{~W})-\mathrm{Na}(8)-\mathrm{Na}(17)$ & $123.6(7)$ & $\mathrm{O}(41 \mathrm{~W})-\mathrm{Na}(8)-\mathrm{Na}(17)$ & $45.0(6)$ \\
\hline $\mathrm{O}(28 \mathrm{~W})-\mathrm{Na}(8)-\mathrm{Na}(17)$ & $91.2(5)$ & $\mathrm{Na}(17) \# 6-\mathrm{Na}(8)-\mathrm{Na}(17)$ & $116.6(5)$ \\
\hline $\mathrm{P}(12)-\mathrm{Na}(8)-\mathrm{Na}(17)$ & $59.1(5)$ & $\mathrm{P}(12) \# 6-\mathrm{Na}(8)-\mathrm{Na}(17)$ & $86.2(4)$ \\
\hline $\mathrm{O}(80) \# 6-\mathrm{Na}(8)-\mathrm{Na}(8) \# 6$ & $41.4(3)$ & $\mathrm{O}(24 \mathrm{~W})-\mathrm{Na}(8)-\mathrm{Na}(8) \# 6$ & $92.7(6)$ \\
\hline $\mathrm{O}(80)-\mathrm{Na}(8)-\mathrm{Na}(8) \# 6$ & $40.5(3)$ & $\mathrm{O}(35 \mathrm{~W})-\mathrm{Na}(8)-\mathrm{Na}(8) \# 6$ & $148.2(7)$ \\
\hline $\mathrm{O}(41 \mathrm{~W})-\mathrm{Na}(8)-\mathrm{Na}(8) \# 6$ & $91.8(6)$ & $\mathrm{O}(28 \mathrm{~W})-\mathrm{Na}(8)-\mathrm{Na}(8) \# 6$ & $127.7(7)$ \\
\hline $\mathrm{Na}(17) \# 6-\mathrm{Na}(8)-\mathrm{Na}(8) \# 6$ & $60.0(5)$ & $\mathrm{P}(12)-\mathrm{Na}(8)-\mathrm{Na}(8) \# 6$ & $58.8(4)$ \\
\hline $\mathrm{P}(12) \# 6-\mathrm{Na}(8)-\mathrm{Na}(8) \# 6$ & $57.9(3)$ & $\mathrm{Na}(17)-\mathrm{Na}(8)-\mathrm{Na}(8) \# 6$ & $56.6(5)$ \\
\hline $\mathrm{O}(14 \mathrm{~W})-\mathrm{Na}(9)-\mathrm{O}(36 \mathrm{~W})$ & $84.8(5)$ & $\mathrm{O}(14 \mathrm{~W})-\mathrm{Na}(9)-\mathrm{O}(13 \mathrm{~W}) \# 7$ & $169.6(7)$ \\
\hline $\mathrm{O}(36 \mathrm{~W})-\mathrm{Na}(9)-\mathrm{O}(13 \mathrm{~W}) \# 7$ & $105.0(6)$ & $\mathrm{O}(14 \mathrm{~W})-\mathrm{Na}(9)-\mathrm{O}(19 \mathrm{~W})$ & $93.6(5)$ \\
\hline $\mathrm{O}(36 \mathrm{~W})-\mathrm{Na}(9)-\mathrm{O}(19 \mathrm{~W})$ & $88.7(6)$ & $\mathrm{O}(13 \mathrm{~W}) \# 7-\mathrm{Na}(9)-\mathrm{O}(19 \mathrm{~W})$ & $83.6(5)$ \\
\hline $\mathrm{O}(14 \mathrm{~W})-\mathrm{Na}(9)-\mathrm{O}(11 \mathrm{~W}) \# 7$ & $87.5(5)$ & $\mathrm{O}(36 \mathrm{~W})-\mathrm{Na}(9)-\mathrm{O}(11 \mathrm{~W}) \# 7$ & $171.5(7)$ \\
\hline $\mathrm{O}(13 \mathrm{~W}) \# 7-\mathrm{Na}(9)-\mathrm{O}(11 \mathrm{~W}) \# 7$ & $82.5(5)$ & $\mathrm{O}(19 \mathrm{~W})-\mathrm{Na}(9)-\mathrm{O}(11 \mathrm{~W}) \# 7$ & $88.3(5)$ \\
\hline $\mathrm{O}(14 \mathrm{~W})-\mathrm{Na}(9)-\mathrm{O}(26 \mathrm{~W})$ & $84.5(6)$ & $\mathrm{O}(36 \mathrm{~W})-\mathrm{Na}(9)-\mathrm{O}(26 \mathrm{~W})$ & $97.0(6)$ \\
\hline $\mathrm{O}(13 \mathrm{~W}) \# 7-\mathrm{Na}(9)-\mathrm{O}(26 \mathrm{~W})$ & $97.2(5)$ & $\mathrm{O}(19 \mathrm{~W})-\mathrm{Na}(9)-\mathrm{O}(26 \mathrm{~W})$ & $173.8(8)$ \\
\hline $\mathrm{O}(11 \mathrm{~W}) \# 7-\mathrm{Na}(9)-\mathrm{O}(26 \mathrm{~W})$ & $85.8(6)$ & $\mathrm{O}(14 \mathrm{~W})-\mathrm{Na}(9)-\mathrm{Na}(2)$ & $43.0(4)$ \\
\hline $\mathrm{O}(36 \mathrm{~W})-\mathrm{Na}(9)-\mathrm{Na}(2)$ & $41.9(4)$ & $\mathrm{O}(13 \mathrm{~W}) \# 7-\mathrm{Na}(9)-\mathrm{Na}(2)$ & $146.8(6)$ \\
\hline $\mathrm{O}(19 \mathrm{~W})-\mathrm{Na}(9)-\mathrm{Na}(2)$ & $91.3(5)$ & $\mathrm{O}(11 \mathrm{~W}) \# 7-\mathrm{Na}(9)-\mathrm{Na}(2)$ & $130.3(6)$ \\
\hline $\mathrm{O}(26 \mathrm{~W})-\mathrm{Na}(9)-\mathrm{Na}(2)$ & $91.4(5)$ & $\mathrm{O}(14 \mathrm{~W})-\mathrm{Na}(9)-\mathrm{Na}(13) \# 7$ & $128.4(6)$ \\
\hline $\mathrm{O}(36 \mathrm{~W})-\mathrm{Na}(9)-\mathrm{Na}(13) \# 7$ & $144.4(6)$ & $\mathrm{O}(13 \mathrm{~W}) \# 7-\mathrm{Na}(9)-\mathrm{Na}(13) \# 7$ & $41.2(4)$ \\
\hline $\mathrm{O}(19 \mathrm{~W})-\mathrm{Na}(9)-\mathrm{Na}(13) \# 7$ & $78.1(5)$ & $\mathrm{O}(11 \mathrm{~W}) \# 7-\mathrm{Na}(9)-\mathrm{Na}(13) \# 7$ & $42.0(4)$ \\
\hline $\mathrm{O}(26 \mathrm{~W})-\mathrm{Na}(9)-\mathrm{Na}(13) \# 7$ & $98.4(5)$ & $\mathrm{Na}(2)-\mathrm{Na}(9)-\mathrm{Na}(13) \# 7$ & $166.4(5)$ \\
\hline $\mathrm{O}(8 \mathrm{~W})-\mathrm{Na}(10)-\mathrm{O}(31 \mathrm{~W})$ & $98.4(7)$ & $\mathrm{O}(8 \mathrm{~W})-\mathrm{Na}(10)-\mathrm{O}(21 \mathrm{~W})$ & $168.2(8)$ \\
\hline $\mathrm{O}(31 \mathrm{~W})-\mathrm{Na}(10)-\mathrm{O}(21 \mathrm{~W})$ & $87.9(6)$ & $\mathrm{O}(8 \mathrm{~W})-\mathrm{Na}(10)-\mathrm{O}(72)$ & $86.0(5)$ \\
\hline $\mathrm{O}(31 \mathrm{~W})-\mathrm{Na}(10)-\mathrm{O}(72)$ & $164.5(8)$ & $\mathrm{O}(21 \mathrm{~W})-\mathrm{Na}(10)-\mathrm{O}(72)$ & $85.4(6)$ \\
\hline $\mathrm{O}(8 \mathrm{~W})-\mathrm{Na}(10)-\mathrm{O}(22 \mathrm{~W})$ & $87.9(6)$ & $\mathrm{O}(31 \mathrm{~W})-\mathrm{Na}(10)-\mathrm{O}(22 \mathrm{~W})$ & $84.4(6)$ \\
\hline $\mathrm{O}(21 \mathrm{~W})-\mathrm{Na}(10)-\mathrm{O}(22 \mathrm{~W})$ & $102.7(6)$ & $\mathrm{O}(72)-\mathrm{Na}(10)-\mathrm{O}(22 \mathrm{~W})$ & $110.6(6)$ \\
\hline $\mathrm{O}(8 \mathrm{~W})-\mathrm{Na}(10)-\mathrm{O}(72) \# 3$ & $84.5(5)$ & $\mathrm{O}(31 \mathrm{~W})-\mathrm{Na}(10)-\mathrm{O}(72) \# 3$ & $80.3(5)$ \\
\hline $\mathrm{O}(21 \mathrm{~W})-\mathrm{Na}(10)-\mathrm{O}(72) \# 3$ & $86.7(6)$ & $\mathrm{O}(72)-\mathrm{Na}(10)-\mathrm{O}(72) \# 3$ & $85.5(5)$ \\
\hline $\mathrm{O}(22 \mathrm{~W})-\mathrm{Na}(10)-\mathrm{O}(72) \# 3$ & $161.7(7)$ & $\mathrm{O}(8 \mathrm{~W})-\mathrm{Na}(10)-\mathrm{Na}(15) \# 3$ & $127.1(6)$ \\
\hline $\mathrm{O}(31 \mathrm{~W})-\mathrm{Na}(10)-\mathrm{Na}(15) \# 3$ & $76.6(5)$ & $\mathrm{O}(21 \mathrm{~W})-\mathrm{Na}(10)-\mathrm{Na}(15) \# 3$ & $44.7(4)$ \\
\hline $\mathrm{O}(72)-\mathrm{Na}(10)-\mathrm{Na}(15) \# 3$ & $88.9(5)$ & $\mathrm{O}(22 \mathrm{~W})-\mathrm{Na}(10)-\mathrm{Na}(15) \# 3$ & $141.9(6)$ \\
\hline $\mathrm{O}(72) \# 3-\mathrm{Na}(10)-\mathrm{Na}(15) \# 3$ & $42.6(4)$ & $\mathrm{O}(8 \mathrm{~W})-\mathrm{Na}(10)-\mathrm{Na}(15)$ & $44.0(4)$ \\
\hline $\mathrm{O}(31 \mathrm{~W})-\mathrm{Na}(10)-\mathrm{Na}(15)$ & $141.3(7)$ & $\mathrm{O}(21 \mathrm{~W})-\mathrm{Na}(10)-\mathrm{Na}(15)$ & $127.6(7)$ \\
\hline $\mathrm{O}(72)-\mathrm{Na}(10)-\mathrm{Na}(15)$ & $42.3(4)$ & $\mathrm{O}(22 \mathrm{~W})-\mathrm{Na}(10)-\mathrm{Na}(15)$ & $99.6(6)$ \\
\hline $\mathrm{O}(72) \# 3-\mathrm{Na}(10)-\mathrm{Na}(15)$ & $86.4(5)$ & $\mathrm{Na}(15) \# 3-\mathrm{Na}(10)-\mathrm{Na}(15)$ & $115.9(5)$ \\
\hline $\mathrm{O}(8 \mathrm{~W})-\mathrm{Na}(10)-\mathrm{Na}(10) \# 3$ & $83.5(6)$ & $\mathrm{O}(31 \mathrm{~W})-\mathrm{Na}(10)-\mathrm{Na}(10) \# 3$ & $121.2(7)$ \\
\hline $\mathrm{O}(21 \mathrm{~W})-\mathrm{Na}(10)-\mathrm{Na}(10) \# 3$ & $84.7(6)$ & $\mathrm{O}(72)-\mathrm{Na}(10)-\mathrm{Na}(10) \# 3$ & $44.2(4)$ \\
\hline $\mathrm{O}(22 \mathrm{~W})-\mathrm{Na}(10)-\mathrm{Na}(10) \# 3$ & $153.8(8)$ & $\mathrm{O}(72) \# 3-\mathrm{Na}(10)-\mathrm{Na}(10) \# 3$ & $41.2(3)$ \\
\hline $\mathrm{Na}(15) \# 3-\mathrm{Na}(10)-\mathrm{Na}(10) \# 3$ & $58.1(5)$ & $\mathrm{Na}(15)-\mathrm{Na}(10)-\mathrm{Na}(10) \# 3$ & $57.8(4)$ \\
\hline $\mathrm{O}(8 \mathrm{~W})-\mathrm{Na}(10)-\mathrm{K}(2) \# 3$ & $63.9(4)$ & $\mathrm{O}(31 \mathrm{~W})-\mathrm{Na}(10)-\mathrm{K}(2) \# 3$ & $35.0(4)$ \\
\hline $\mathrm{O}(21 \mathrm{~W})-\mathrm{Na}(10)-\mathrm{K}(2) \# 3$ & $120.5(5)$ & $\mathrm{O}(72)-\mathrm{Na}(10)-\mathrm{K}(2) \# 3$ & $143.5(5)$ \\
\hline $\mathrm{O}(22 \mathrm{~W})-\mathrm{Na}(10)-\mathrm{K}(2) \# 3$ & $89.6(5)$ & $\mathrm{O}(72) \# 3-\mathrm{Na}(10)-\mathrm{K}(2) \# 3$ & $72.1(4)$ \\
\hline $\mathrm{Na}(15) \# 3-\mathrm{Na}(10)-\mathrm{K}(2) \# 3$ & $93.1(4)$ & $\mathrm{Na}(15)-\mathrm{Na}(10)-\mathrm{K}(2) \# 3$ & $106.3(5)$ \\
\hline
\end{tabular}




\begin{tabular}{|c|c|c|c|}
\hline $\mathrm{Na}(10) \# 3-\mathrm{Na}(10)-\mathrm{K}(2) \# 3$ & $108.4(5)$ & $\mathrm{O}(32 \mathrm{~W})-\mathrm{Na}(11)-\mathrm{O}(6 \mathrm{~W})$ & $98.6(6)$ \\
\hline $\mathrm{O}(32 \mathrm{~W})-\mathrm{Na}(11)-\mathrm{O}(54) \# 1$ & $131.1(7)$ & $\mathrm{O}(6 \mathrm{~W})-\mathrm{Na}(11)-\mathrm{O}(54) \# 1$ & $89.5(6)$ \\
\hline $\mathrm{O}(32 \mathrm{~W})-\mathrm{Na}(11)-\mathrm{O}(12)$ & $148.1(8)$ & $\mathrm{O}(6 \mathrm{~W})-\mathrm{Na}(11)-\mathrm{O}(12)$ & $94.9(6)$ \\
\hline $\mathrm{O}(54) \# 1-\mathrm{Na}(11)-\mathrm{O}(12)$ & $77.5(5)$ & $\mathrm{O}(32 \mathrm{~W})-\mathrm{Na}(11)-\mathrm{O}(64 \mathrm{~W})$ & $84.5(5)$ \\
\hline $\mathrm{O}(6 \mathrm{~W})-\mathrm{Na}(11)-\mathrm{O}(64 \mathrm{~W})$ & $171.0(8)$ & $\mathrm{O}(54) \# 1-\mathrm{Na}(11)-\mathrm{O}(64 \mathrm{~W})$ & $82.1(5)$ \\
\hline $\mathrm{O}(12)-\mathrm{Na}(11)-\mathrm{O}(64 \mathrm{~W})$ & $86.6(6)$ & $\mathrm{O}(32 \mathrm{~W})-\mathrm{Na}(11)-\mathrm{O}(25)$ & $84.9(5)$ \\
\hline $\mathrm{O}(6 \mathrm{~W})-\mathrm{Na}(11)-\mathrm{O}(25)$ & $83.7(5)$ & $\mathrm{O}(54) \# 1-\mathrm{Na}(11)-\mathrm{O}(25)$ & $144.0(7)$ \\
\hline $\mathrm{O}(12)-\mathrm{Na}(11)-\mathrm{O}(25)$ & $67.9(5)$ & $\mathrm{O}(64 \mathrm{~W})-\mathrm{Na}(11)-\mathrm{O}(25)$ & $105.0(6)$ \\
\hline $\mathrm{O}(32 \mathrm{~W})-\mathrm{Na}(11)-\mathrm{H}(64 \mathrm{~A})$ & $75.8(9)$ & $\mathrm{O}(6 \mathrm{~W})-\mathrm{Na}(11)-\mathrm{H}(64 \mathrm{~A})$ & 166.7(9) \\
\hline $\mathrm{O}(54) \# 1-\mathrm{Na}(11)-\mathrm{H}(64 \mathrm{~A})$ & $103.3(7)$ & $\mathrm{O}(12)-\mathrm{Na}(11)-\mathrm{H}(64 \mathrm{~A})$ & $84.5(10)$ \\
\hline $\mathrm{O}(64 \mathrm{~W})-\mathrm{Na}(11)-\mathrm{H}(64 \mathrm{~A})$ & $22.2(4)$ & $\mathrm{O}(25)-\mathrm{Na}(11)-\mathrm{H}(64 \mathrm{~A})$ & $83.8(7)$ \\
\hline $\mathrm{O}(32 \mathrm{~W})-\mathrm{Na}(11)-\mathrm{H}(32 \mathrm{C})$ & $21.5(4)$ & $\mathrm{O}(6 \mathrm{~W})-\mathrm{Na}(11)-\mathrm{H}(32 \mathrm{C})$ & $82.5(8)$ \\
\hline $\mathrm{O}(54) \# 1-\mathrm{Na}(11)-\mathrm{H}(32 \mathrm{C})$ & $117.0(9)$ & $\mathrm{O}(12)-\mathrm{Na}(11)-\mathrm{H}(32 \mathrm{C})$ & $165.1(10)$ \\
\hline $\mathrm{O}(64 \mathrm{~W})-\mathrm{Na}(11)-\mathrm{H}(32 \mathrm{C})$ & $98.2(9)$ & $\mathrm{O}(25)-\mathrm{Na}(11)-\mathrm{H}(32 \mathrm{C})$ & $97.2(9)$ \\
\hline $\mathrm{H}(64 \mathrm{~A})-\mathrm{Na}(11)-\mathrm{H}(32 \mathrm{C})$ & $94.6(11)$ & $\mathrm{O}(32 \mathrm{~W})-\mathrm{Na}(11)-\mathrm{H}(64 \mathrm{D})$ & $103.9(7)$ \\
\hline $\mathrm{O}(6 \mathrm{~W})-\mathrm{Na}(11)-\mathrm{H}(64 \mathrm{D})$ & $157.4(8)$ & $\mathrm{O}(54) \# 1-\mathrm{Na}(11)-\mathrm{H}(64 \mathrm{D})$ & $77.0(9)$ \\
\hline $\mathrm{O}(12)-\mathrm{Na}(11)-\mathrm{H}(64 \mathrm{D})$ & $64.8(6)$ & $\mathrm{O}(64 \mathrm{~W})-\mathrm{Na}(11)-\mathrm{H}(64 \mathrm{D})$ & $21.9(4)$ \\
\hline $\mathrm{O}(25)-\mathrm{Na}(11)-\mathrm{H}(64 \mathrm{D})$ & $96.6(9)$ & $\mathrm{H}(64 \mathrm{~A})-\mathrm{Na}(11)-\mathrm{H}(64 \mathrm{D})$ & $30.0(9)$ \\
\hline $\mathrm{H}(32 \mathrm{C})-\mathrm{Na}(11)-\mathrm{H}(64 \mathrm{D})$ & 119.7(9) & $\mathrm{O}(32 \mathrm{~W})-\mathrm{Na}(11)-\mathrm{H}(6 \mathrm{~B})$ & $112.2(8)$ \\
\hline $\mathrm{O}(6 \mathrm{~W})-\mathrm{Na}(11)-\mathrm{H}(6 \mathrm{~B})$ & $21.0(6)$ & $\mathrm{O}(54) \# 1-\mathrm{Na}(11)-\mathrm{H}(6 \mathrm{~B})$ & $91.8(6)$ \\
\hline $\mathrm{O}(12)-\mathrm{Na}(11)-\mathrm{H}(6 \mathrm{~B})$ & $75.1(6)$ & $\mathrm{O}(64 \mathrm{~W})-\mathrm{Na}(11)-\mathrm{H}(6 \mathrm{~B})$ & $161.6(8)$ \\
\hline $\mathrm{O}(25)-\mathrm{Na}(11)-\mathrm{H}(6 \mathrm{~B})$ & $70.1(6)$ & $\mathrm{H}(64 \mathrm{~A})-\mathrm{Na}(11)-\mathrm{H}(6 \mathrm{~B})$ & $151.4(10)$ \\
\hline $\mathrm{H}(32 \mathrm{C})-\mathrm{Na}(11)-\mathrm{H}(6 \mathrm{~B})$ & $100.0(10)$ & $\mathrm{H}(64 \mathrm{D})-\mathrm{Na}(11)-\mathrm{H}(6 \mathrm{~B})$ & $139.7(8)$ \\
\hline $\mathrm{O}(32 \mathrm{~W})-\mathrm{Na}(11)-\mathrm{O}(61 \mathrm{~W}) \# 1$ & $70.7(6)$ & $\mathrm{O}(6 \mathrm{~W})-\mathrm{Na}(11)-\mathrm{O}(61 \mathrm{~W}) \# 1$ & $90.6(6)$ \\
\hline $\mathrm{O}(54) \# 1-\mathrm{Na}(11)-\mathrm{O}(61 \mathrm{~W}) \# 1$ & $61.0(5)$ & $\mathrm{O}(12)-\mathrm{Na}(11)-\mathrm{O}(61 \mathrm{~W}) \# 1$ & $138.1(7)$ \\
\hline $\mathrm{O}(64 \mathrm{~W})-\mathrm{Na}(11)-\mathrm{O}(61 \mathrm{~W}) \# 1$ & $82.4(6)$ & $\mathrm{O}(25)-\mathrm{Na}(11)-\mathrm{O}(61 \mathrm{~W}) \# 1$ & $153.9(7)$ \\
\hline $\mathrm{H}(64 \mathrm{~A})-\mathrm{Na}(11)-\mathrm{O}(61 \mathrm{~W}) \# 1$ & $98.6(9)$ & $\mathrm{H}(32 \mathrm{C})-\mathrm{Na}(11)-\mathrm{O}(61 \mathrm{~W}) \# 1$ & $56.8(9)$ \\
\hline $\mathrm{H}(64 \mathrm{D})-\mathrm{Na}(11)-\mathrm{O}(61 \mathrm{~W}) \# 1$ & $98.2(8)$ & $\mathrm{H}(6 \mathrm{~B})-\mathrm{Na}(11)-\mathrm{O}(61 \mathrm{~W}) \# 1$ & $110.0(8)$ \\
\hline $\mathrm{O}(32 \mathrm{~W})-\mathrm{Na}(11)-\mathrm{U}(2)$ & $155.5(6)$ & $\mathrm{O}(6 \mathrm{~W})-\mathrm{Na}(11)-\mathrm{U}(2)$ & $77.5(4)$ \\
\hline $\mathrm{O}(54) \# 1-\mathrm{Na}(11)-\mathrm{U}(2)$ & $73.4(4)$ & $\mathrm{O}(12)-\mathrm{Na}(11)-\mathrm{U}(2)$ & $18.0(2)$ \\
\hline $\mathrm{O}(64 \mathrm{~W})-\mathrm{Na}(11)-\mathrm{U}(2)$ & $103.1(5)$ & $\mathrm{O}(25)-\mathrm{Na}(11)-\mathrm{U}(2)$ & $70.6(4)$ \\
\hline $\mathrm{H}(64 \mathrm{~A})-\mathrm{Na}(11)-\mathrm{U}(2)$ & $102.5(10)$ & $\mathrm{H}(32 \mathrm{C})-\mathrm{Na}(11)-\mathrm{U}(2)$ & $157.5(9)$ \\
\hline $\mathrm{H}(64 \mathrm{D})-\mathrm{Na}(11)-\mathrm{U}(2)$ & $81.3(5)$ & $\mathrm{H}(6 \mathrm{~B})-\mathrm{Na}(11)-\mathrm{U}(2)$ & $58.4(5)$ \\
\hline $\mathrm{O}(61 \mathrm{~W}) \# 1-\mathrm{Na}(11)-\mathrm{U}(2)$ & $132.9(6)$ & $\mathrm{O}(\mathrm{OW})-\mathrm{Na}(12)-\mathrm{O}(3 \mathrm{~W})$ & $149.5(8)$ \\
\hline $\mathrm{O}(\mathrm{OW})-\mathrm{Na}(12)-\mathrm{O}(1 \mathrm{~W})$ & $107.8(7)$ & $\mathrm{O}(3 \mathrm{~W})-\mathrm{Na}(12)-\mathrm{O}(1 \mathrm{~W})$ & $102.4(6)$ \\
\hline $\mathrm{O}(\mathrm{OW})-\mathrm{Na}(12)-\mathrm{O}(2 \mathrm{~W})$ & $84.8(6)$ & $\mathrm{O}(3 \mathrm{~W})-\mathrm{Na}(12)-\mathrm{O}(2 \mathrm{~W})$ & $91.6(7)$ \\
\hline $\mathrm{O}(1 \mathrm{~W})-\mathrm{Na}(12)-\mathrm{O}(2 \mathrm{~W})$ & $88.2(6)$ & $\mathrm{O}(0 \mathrm{~W})-\mathrm{Na}(12)-\mathrm{O}(89)$ & $83.7(5)$ \\
\hline $\mathrm{O}(3 \mathrm{~W})-\mathrm{Na}(12)-\mathrm{O}(89)$ & $94.0(6)$ & $\mathrm{O}(1 \mathrm{~W})-\mathrm{Na}(12)-\mathrm{O}(89)$ & $103.4(6)$ \\
\hline $\mathrm{O}(2 \mathrm{~W})-\mathrm{Na}(12)-\mathrm{O}(89)$ & $165.7(7)$ & $\mathrm{O}(0 \mathrm{~W})-\mathrm{Na}(12)-\mathrm{O}(82)$ & $73.6(5)$ \\
\hline $\mathrm{O}(3 \mathrm{~W})-\mathrm{Na}(12)-\mathrm{O}(82)$ & $80.6(5)$ & $\mathrm{O}(1 \mathrm{~W})-\mathrm{Na}(12)-\mathrm{O}(82)$ & $157.0(6)$ \\
\hline $\mathrm{O}(2 \mathrm{~W})-\mathrm{Na}(12)-\mathrm{O}(82)$ & $114.7(6)$ & $\mathrm{O}(89)-\mathrm{Na}(12)-\mathrm{O}(82)$ & $53.6(4)$ \\
\hline $\mathrm{O}(\mathrm{OW})-\mathrm{Na}(12)-\mathrm{P}(5)$ & $80.0(5)$ & $\mathrm{O}(3 \mathrm{~W})-\mathrm{Na}(12)-\mathrm{P}(5)$ & $85.4(5)$ \\
\hline $\mathrm{O}(1 \mathrm{~W})-\mathrm{Na}(12)-\mathrm{P}(5)$ & $127.7(6)$ & $\mathrm{O}(2 \mathrm{~W})-\mathrm{Na}(12)-\mathrm{P}(5)$ & $143.7(6)$ \\
\hline $\mathrm{O}(89)-\mathrm{Na}(12)-\mathrm{P}(5)$ & $24.5(3)$ & $\mathrm{O}(82)-\mathrm{Na}(12)-\mathrm{P}(5)$ & $29.2(2)$ \\
\hline
\end{tabular}




\begin{tabular}{|c|c|c|c|}
\hline $\mathrm{O}(\mathrm{OW})-\mathrm{Na}(12)-\mathrm{Na}(2) \# 8$ & $96.8(6)$ & $\mathrm{O}(3 \mathrm{~W})-\mathrm{Na}(12)-\mathrm{Na}(2) \# 8$ & $110.6(6)$ \\
\hline $\mathrm{O}(1 \mathrm{~W})-\mathrm{Na}(12)-\mathrm{Na}(2) \# 8$ & $35.9(4)$ & $\mathrm{O}(2 \mathrm{~W})-\mathrm{Na}(12)-\mathrm{Na}(2) \# 8$ & $121.9(6)$ \\
\hline $\mathrm{O}(89)-\mathrm{Na}(12)-\mathrm{Na}(2) \# 8$ & $68.1(4)$ & $\mathrm{O}(82)-\mathrm{Na}(12)-\mathrm{Na}(2) \# 8$ & $121.4(5)$ \\
\hline $\mathrm{P}(5)-\mathrm{Na}(12)-\mathrm{Na}(2) \# 8$ & $92.6(4)$ & $\mathrm{O}(60)-\mathrm{Na}(13)-\mathrm{O}(30 \mathrm{~W})$ & $82.7(6)$ \\
\hline $\mathrm{O}(60)-\mathrm{Na}(13)-\mathrm{O}(13 \mathrm{~W})$ & $111.8(7)$ & $\mathrm{O}(30 \mathrm{~W})-\mathrm{Na}(13)-\mathrm{O}(13 \mathrm{~W})$ & $163.6(8)$ \\
\hline $\mathrm{O}(60)-\mathrm{Na}(13)-\mathrm{O}(23 \mathrm{~W})$ & $163.2(7)$ & $\mathrm{O}(30 \mathrm{~W})-\mathrm{Na}(13)-\mathrm{O}(23 \mathrm{~W})$ & $81.2(6)$ \\
\hline $\mathrm{O}(13 \mathrm{~W})-\mathrm{Na}(13)-\mathrm{O}(23 \mathrm{~W})$ & $84.8(5)$ & $\mathrm{O}(60)-\mathrm{Na}(13)-\mathrm{O}(11 \mathrm{~W})$ & $89.3(6)$ \\
\hline $\mathrm{O}(30 \mathrm{~W})-\mathrm{Na}(13)-\mathrm{O}(11 \mathrm{~W})$ & $106.4(7)$ & $\mathrm{O}(13 \mathrm{~W})-\mathrm{Na}(13)-\mathrm{O}(11 \mathrm{~W})$ & $82.2(5)$ \\
\hline $\mathrm{O}(23 \mathrm{~W})-\mathrm{Na}(13)-\mathrm{O}(11 \mathrm{~W})$ & $90.3(6)$ & $\mathrm{O}(60)-\mathrm{Na}(13)-\mathrm{O}(29 \mathrm{~W})$ & $75.4(5)$ \\
\hline $\mathrm{O}(30 \mathrm{~W})-\mathrm{Na}(13)-\mathrm{O}(29 \mathrm{~W})$ & $91.9(6)$ & $\mathrm{O}(13 \mathrm{~W})-\mathrm{Na}(13)-\mathrm{O}(29 \mathrm{~W})$ & $84.8(6)$ \\
\hline $\mathrm{O}(23 \mathrm{~W})-\mathrm{Na}(13)-\mathrm{O}(29 \mathrm{~W})$ & $110.1(7)$ & $\mathrm{O}(11 \mathrm{~W})-\mathrm{Na}(13)-\mathrm{O}(29 \mathrm{~W})$ & $154.6(7)$ \\
\hline $\mathrm{O}(60)-\mathrm{Na}(13)-\mathrm{Na}(18)$ & $77.2(6)$ & $\mathrm{O}(30 \mathrm{~W})-\mathrm{Na}(13)-\mathrm{Na}(18)$ & $41.6(5)$ \\
\hline $\mathrm{O}(13 \mathrm{~W})-\mathrm{Na}(13)-\mathrm{Na}(18)$ & $131.8(7)$ & $\mathrm{O}(23 \mathrm{~W})-\mathrm{Na}(13)-\mathrm{Na}(18)$ & $94.0(7)$ \\
\hline $\mathrm{O}(11 \mathrm{~W})-\mathrm{Na}(13)-\mathrm{Na}(18)$ & $146.0(7)$ & $\mathrm{O}(29 \mathrm{~W})-\mathrm{Na}(13)-\mathrm{Na}(18)$ & $50.4(5)$ \\
\hline $\mathrm{O}(60)-\mathrm{Na}(13)-\mathrm{Na}(9) \# 7$ & $110.0(6)$ & $\mathrm{O}(30 \mathrm{~W})-\mathrm{Na}(13)-\mathrm{Na}(9) \# 7$ & $142.8(7)$ \\
\hline $\mathrm{O}(13 \mathrm{~W})-\mathrm{Na}(13)-\mathrm{Na}(9) \# 7$ & $41.0(4)$ & $\mathrm{O}(23 \mathrm{~W})-\mathrm{Na}(13)-\mathrm{Na}(9) \# 7$ & $80.4(5)$ \\
\hline $\mathrm{O}(11 \mathrm{~W})-\mathrm{Na}(13)-\mathrm{Na}(9) \# 7$ & $42.0(4)$ & $\mathrm{O}(29 \mathrm{~W})-\mathrm{Na}(13)-\mathrm{Na}(9) \# 7$ & $124.8(6)$ \\
\hline $\mathrm{Na}(18)-\mathrm{Na}(13)-\mathrm{Na}(9) \# 7$ & $170.8(7)$ & $\mathrm{O}(60)-\mathrm{Na}(13)-\mathrm{U}(10)$ & $14.6(3)$ \\
\hline $\mathrm{O}(30 \mathrm{~W})-\mathrm{Na}(13)-\mathrm{U}(10)$ & $93.9(5)$ & $\mathrm{O}(13 \mathrm{~W})-\mathrm{Na}(13)-\mathrm{U}(10)$ & $101.7(5)$ \\
\hline $\mathrm{O}(23 \mathrm{~W})-\mathrm{Na}(13)-\mathrm{U}(10)$ & $164.8(7)$ & $\mathrm{O}(11 \mathrm{~W})-\mathrm{Na}(13)-\mathrm{U}(10)$ & $77.1(4)$ \\
\hline $\mathrm{O}(29 \mathrm{~W})-\mathrm{Na}(13)-\mathrm{U}(10)$ & $84.3(4)$ & $\mathrm{Na}(18)-\mathrm{Na}(13)-\mathrm{U}(10)$ & $91.8(6)$ \\
\hline $\mathrm{Na}(9) \# 7-\mathrm{Na}(13)-\mathrm{U}(10)$ & $95.5(4)$ & $\mathrm{O}(42 \mathrm{~W})-\mathrm{Na}(14)-\mathrm{O}(69 \mathrm{~W})$ & 104.3(9) \\
\hline $\mathrm{O}(42 \mathrm{~W})-\mathrm{Na}(14)-\mathrm{O}(16 \mathrm{~W})$ & $101.5(8)$ & $\mathrm{O}(69 \mathrm{~W})-\mathrm{Na}(14)-\mathrm{O}(16 \mathrm{~W})$ & $83.5(7)$ \\
\hline $\mathrm{O}(42 \mathrm{~W})-\mathrm{Na}(14)-\mathrm{O}(69 \mathrm{~V})$ & $92.4(9)$ & $\mathrm{O}(69 \mathrm{~W})-\mathrm{Na}(14)-\mathrm{O}(69 \mathrm{~V})$ & $16.0(6)$ \\
\hline $\mathrm{O}(16 \mathrm{~W})-\mathrm{Na}(14)-\mathrm{O}(69 \mathrm{~V})$ & $96.4(8)$ & $\mathrm{O}(42 \mathrm{~W})-\mathrm{Na}(14)-\mathrm{O}(77)$ & $103.2(8)$ \\
\hline $\mathrm{O}(69 \mathrm{~W})-\mathrm{Na}(14)-\mathrm{O}(77)$ & $152.5(9)$ & $\mathrm{O}(16 \mathrm{~W})-\mathrm{Na}(14)-\mathrm{O}(77)$ & $91.2(7)$ \\
\hline $\mathrm{O}(69 \mathrm{~V})-\mathrm{Na}(14)-\mathrm{O}(77)$ & $160.9(10)$ & $\mathrm{O}(42 \mathrm{~W})-\mathrm{Na}(14)-\mathrm{O}(37 \mathrm{~W})$ & $91.6(7)$ \\
\hline $\mathrm{O}(69 \mathrm{~W})-\mathrm{Na}(14)-\mathrm{O}(37 \mathrm{~W})$ & $92.4(8)$ & $\mathrm{O}(16 \mathrm{~W})-\mathrm{Na}(14)-\mathrm{O}(37 \mathrm{~W})$ & $166.9(9)$ \\
\hline $\mathrm{O}(69 \mathrm{~V})-\mathrm{Na}(14)-\mathrm{O}(37 \mathrm{~W})$ & $81.9(8)$ & $\mathrm{O}(77)-\mathrm{Na}(14)-\mathrm{O}(37 \mathrm{~W})$ & $86.6(6)$ \\
\hline $\mathrm{O}(42 \mathrm{~W})-\mathrm{Na}(14)-\mathrm{O}(27 \mathrm{~W})$ & $174.4(9)$ & $\mathrm{O}(69 \mathrm{~W})-\mathrm{Na}(14)-\mathrm{O}(27 \mathrm{~W})$ & $70.3(7)$ \\
\hline $\mathrm{O}(16 \mathrm{~W})-\mathrm{Na}(14)-\mathrm{O}(27 \mathrm{~W})$ & $80.0(5)$ & $\mathrm{O}(69 \mathrm{~V})-\mathrm{Na}(14)-\mathrm{O}(27 \mathrm{~W})$ & $82.0(8)$ \\
\hline $\mathrm{O}(77)-\mathrm{Na}(14)-\mathrm{O}(27 \mathrm{~W})$ & $82.2(5)$ & $\mathrm{O}(37 \mathrm{~W})-\mathrm{Na}(14)-\mathrm{O}(27 \mathrm{~W})$ & $86.9(7)$ \\
\hline $\mathrm{O}(42 \mathrm{~W})-\mathrm{Na}(14)-\mathrm{Na}(4) \# 5$ & $101.3(8)$ & $\mathrm{O}(69 \mathrm{~W})-\mathrm{Na}(14)-\mathrm{Na}(4) \# 5$ & $126.5(8)$ \\
\hline $\mathrm{O}(16 \mathrm{~W})-\mathrm{Na}(14)-\mathrm{Na}(4) \# 5$ & $45.5(4)$ & $\mathrm{O}(69 \mathrm{~V})-\mathrm{Na}(14)-\mathrm{Na}(4) \# 5$ & $141.2(9)$ \\
\hline $\mathrm{O}(77)-\mathrm{Na}(14)-\mathrm{Na}(4) \# 5$ & $46.5(4)$ & $\mathrm{O}(37 \mathrm{~W})-\mathrm{Na}(14)-\mathrm{Na}(4) \# 5$ & $133.0(7)$ \\
\hline $\mathrm{O}(27 \mathrm{~W})-\mathrm{Na}(14)-\mathrm{Na}(4) \# 5$ & $83.6(5)$ & $\mathrm{O}(42 \mathrm{~W})-\mathrm{Na}(14)-\mathrm{P}(11)$ & $84.7(6)$ \\
\hline $\mathrm{O}(69 \mathrm{~W})-\mathrm{Na}(14)-\mathrm{P}(11)$ & $165.2(9)$ & $\mathrm{O}(16 \mathrm{~W})-\mathrm{Na}(14)-\mathrm{P}(11)$ & $106.4(6)$ \\
\hline $\mathrm{O}(69 \mathrm{~V})-\mathrm{Na}(14)-\mathrm{P}(11)$ & 157.1(9) & $\mathrm{O}(77)-\mathrm{Na}(14)-\mathrm{P}(11)$ & $21.8(3)$ \\
\hline $\mathrm{O}(37 \mathrm{~W})-\mathrm{Na}(14)-\mathrm{P}(11)$ & $75.5(5)$ & $\mathrm{O}(27 \mathrm{~W})-\mathrm{Na}(14)-\mathrm{P}(11)$ & $100.1(5)$ \\
\hline $\mathrm{Na}(4) \# 5-\mathrm{Na}(14)-\mathrm{P}(11)$ & $61.2(4)$ & $\mathrm{O}(42 \mathrm{~W})-\mathrm{Na}(14)-\mathrm{Na}(4)$ & $143.5(8)$ \\
\hline $\mathrm{O}(69 \mathrm{~W})-\mathrm{Na}(14)-\mathrm{Na}(4)$ & $111.9(8)$ & $\mathrm{O}(16 \mathrm{~W})-\mathrm{Na}(14)-\mathrm{Na}(4)$ & $86.7(5)$ \\
\hline $\mathrm{O}(69 \mathrm{~V})-\mathrm{Na}(14)-\mathrm{Na}(4)$ & $122.2(8)$ & $\mathrm{O}(77)-\mathrm{Na}(14)-\mathrm{Na}(4)$ & $40.6(4)$ \\
\hline $\mathrm{O}(37 \mathrm{~W})-\mathrm{Na}(14)-\mathrm{Na}(4)$ & $83.3(6)$ & $\mathrm{O}(27 \mathrm{~W})-\mathrm{Na}(14)-\mathrm{Na}(4)$ & $41.7(4)$ \\
\hline $\mathrm{Na}(4) \# 5-\mathrm{Na}(14)-\mathrm{Na}(4)$ & $59.8(4)$ & $\mathrm{P}(11)-\mathrm{Na}(14)-\mathrm{Na}(4)$ & $59.0(3)$ \\
\hline
\end{tabular}




\begin{tabular}{|c|c|c|c|}
\hline $\mathrm{O}(72)-\mathrm{Na}(15)-\mathrm{O}(38 \mathrm{~W})$ & $107.0(7)$ & $\mathrm{O}(72)-\mathrm{Na}(15)-\mathrm{O}(8 \mathrm{~W})$ & $86.3(5)$ \\
\hline $\mathrm{O}(38 \mathrm{~W})-\mathrm{Na}(15)-\mathrm{O}(8 \mathrm{~W})$ & $166.7(7)$ & $\mathrm{O}(72)-\mathrm{Na}(15)-\mathrm{O}(17 \mathrm{~W})$ & $164.6(8)$ \\
\hline $\mathrm{O}(38 \mathrm{~W})-\mathrm{Na}(15)-\mathrm{O}(17 \mathrm{~W})$ & $84.7(6)$ & $\mathrm{O}(8 \mathrm{~W})-\mathrm{Na}(15)-\mathrm{O}(17 \mathrm{~W})$ & $82.3(6)$ \\
\hline $\mathrm{O}(72)-\mathrm{Na}(15)-\mathrm{O}(21 \mathrm{~W}) \# 3$ & $92.0(5)$ & $\mathrm{O}(38 \mathrm{~W})-\mathrm{Na}(15)-\mathrm{O}(21 \mathrm{~W}) \# 3$ & $96.7(7)$ \\
\hline $\mathrm{O}(8 \mathrm{~W})-\mathrm{Na}(15)-\mathrm{O}(21 \mathrm{~W}) \# 3$ & $82.3(5)$ & $\mathrm{O}(17 \mathrm{~W})-\mathrm{Na}(15)-\mathrm{O}(21 \mathrm{~W}) \# 3$ & $96.8(6)$ \\
\hline $\mathrm{O}(72)-\mathrm{Na}(15)-\mathrm{O}(29 \mathrm{~W})$ & $89.5(5)$ & $\mathrm{O}(38 \mathrm{~W})-\mathrm{Na}(15)-\mathrm{O}(29 \mathrm{~W})$ & $86.3(6)$ \\
\hline $\mathrm{O}(8 \mathrm{~W})-\mathrm{Na}(15)-\mathrm{O}(29 \mathrm{~W})$ & $94.2(6)$ & $\mathrm{O}(17 \mathrm{~W})-\mathrm{Na}(15)-\mathrm{O}(29 \mathrm{~W})$ & $81.0(5)$ \\
\hline $\mathrm{O}(21 \mathrm{~W}) \# 3-\mathrm{Na}(15)-\mathrm{O}(29 \mathrm{~W})$ & $176.1(8)$ & $\mathrm{O}(72)-\mathrm{Na}(15)-\mathrm{Na}(18)$ & $135.4(7)$ \\
\hline $\mathrm{O}(38 \mathrm{~W})-\mathrm{Na}(15)-\mathrm{Na}(18)$ & $52.7(7)$ & $\mathrm{O}(8 \mathrm{~W})-\mathrm{Na}(15)-\mathrm{Na}(18)$ & $117.6(7)$ \\
\hline $\mathrm{O}(17 \mathrm{~W})-\mathrm{Na}(15)-\mathrm{Na}(18)$ & $45.2(6)$ & $\mathrm{O}(21 \mathrm{~W}) \# 3-\mathrm{Na}(15)-\mathrm{Na}(18)$ & $126.2(7)$ \\
\hline $\mathrm{O}(29 \mathrm{~W})-\mathrm{Na}(15)-\mathrm{Na}(18)$ & $54.1(6)$ & $\mathrm{O}(72)-\mathrm{Na}(15)-\mathrm{Na}(10) \# 3$ & $48.1(4)$ \\
\hline $\mathrm{O}(38 \mathrm{~W})-\mathrm{Na}(15)-\mathrm{Na}(10) \# 3$ & $101.2(7)$ & $\mathrm{O}(8 \mathrm{~W})-\mathrm{Na}(15)-\mathrm{Na}(10) \# 3$ & $87.4(5)$ \\
\hline $\mathrm{O}(17 \mathrm{~W})-\mathrm{Na}(15)-\mathrm{Na}(10) \# 3$ & $141.0(6)$ & $\mathrm{O}(21 \mathrm{~W}) \# 3-\mathrm{Na}(15)-\mathrm{Na}(10) \# 3$ & $44.4(4)$ \\
\hline $\mathrm{O}(29 \mathrm{~W})-\mathrm{Na}(15)-\mathrm{Na}(10) \# 3$ & $137.4(6)$ & $\mathrm{Na}(18)-\mathrm{Na}(15)-\mathrm{Na}(10) \# 3$ & $153.6(7)$ \\
\hline $\mathrm{O}(72)-\mathrm{Na}(15)-\mathrm{P}(2)$ & $20.1(3)$ & $\mathrm{O}(38 \mathrm{~W})-\mathrm{Na}(15)-\mathrm{P}(2)$ & $90.6(5)$ \\
\hline $\mathrm{O}(8 \mathrm{~W})-\mathrm{Na}(15)-\mathrm{P}(2)$ & $102.4(5)$ & $\mathrm{O}(17 \mathrm{~W})-\mathrm{Na}(15)-\mathrm{P}(2)$ & $157.3(6)$ \\
\hline $\mathrm{O}(21 \mathrm{~W}) \# 3-\mathrm{Na}(15)-\mathrm{P}(2)$ & $105.8(5)$ & $\mathrm{O}(29 \mathrm{~W})-\mathrm{Na}(15)-\mathrm{P}(2)$ & $76.6(4)$ \\
\hline $\mathrm{Na}(18)-\mathrm{Na}(15)-\mathrm{P}(2)$ & $116.0(6)$ & $\mathrm{Na}(10) \# 3-\mathrm{Na}(15)-\mathrm{P}(2)$ & $61.6(4)$ \\
\hline $\mathrm{O}(72)-\mathrm{Na}(15)-\mathrm{Na}(10)$ & $44.3(4)$ & $\mathrm{O}(38 \mathrm{~W})-\mathrm{Na}(15)-\mathrm{Na}(10)$ & $151.1(7)$ \\
\hline $\mathrm{O}(8 \mathrm{~W})-\mathrm{Na}(15)-\mathrm{Na}(10)$ & $42.2(4)$ & $\mathrm{O}(17 \mathrm{~W})-\mathrm{Na}(15)-\mathrm{Na}(10)$ & $122.8(6)$ \\
\hline $\mathrm{O}(21 \mathrm{~W}) \# 3-\mathrm{Na}(15)-\mathrm{Na}(10)$ & $89.2(5)$ & $\mathrm{O}(29 \mathrm{~W})-\mathrm{Na}(15)-\mathrm{Na}(10)$ & $89.3(5)$ \\
\hline $\mathrm{Na}(18)-\mathrm{Na}(15)-\mathrm{Na}(10)$ & $140.3(7)$ & $\mathrm{Na}(10) \# 3-\mathrm{Na}(15)-\mathrm{Na}(10)$ & $64.1(5)$ \\
\hline $\mathrm{P}(2)-\mathrm{Na}(15)-\mathrm{Na}(10)$ & $60.6(4)$ & $\mathrm{O}(40 \mathrm{~W})-\mathrm{Na}(16)-\mathrm{O}(40 \mathrm{~W}) \# 9$ & 180.0 \\
\hline $\mathrm{O}(40 \mathrm{~W})-\mathrm{Na}(16)-\mathrm{O}(47 \mathrm{~W})$ & $90.6(4)$ & $\mathrm{O}(40 \mathrm{~W}) \# 9-\mathrm{Na}(16)-\mathrm{O}(47 \mathrm{~W})$ & $89.4(4)$ \\
\hline $\mathrm{O}(40 \mathrm{~W})-\mathrm{Na}(16)-\mathrm{O}(47 \mathrm{~W}) \# 9$ & $89.4(4)$ & $\mathrm{O}(40 \mathrm{~W}) \# 9-\mathrm{Na}(16)-\mathrm{O}(47 \mathrm{~W}) \# 9$ & $90.6(4)$ \\
\hline $\mathrm{O}(47 \mathrm{~W})-\mathrm{Na}(16)-\mathrm{O}(47 \mathrm{~W}) \# 9$ & 180.0 & $\mathrm{O}(40 \mathrm{~W})-\mathrm{Na}(16)-\mathrm{O}(87)$ & $78.3(3)$ \\
\hline $\mathrm{O}(40 \mathrm{~W}) \# 9-\mathrm{Na}(16)-\mathrm{O}(87)$ & $101.7(3)$ & $\mathrm{O}(47 \mathrm{~W})-\mathrm{Na}(16)-\mathrm{O}(87)$ & $92.8(3)$ \\
\hline $\mathrm{O}(47 \mathrm{~W}) \# 9-\mathrm{Na}(16)-\mathrm{O}(87)$ & $87.2(3)$ & $\mathrm{O}(40 \mathrm{~W})-\mathrm{Na}(16)-\mathrm{O}(87) \# 9$ & $101.7(3)$ \\
\hline $\mathrm{O}(40 \mathrm{~W}) \# 9-\mathrm{Na}(16)-\mathrm{O}(87) \# 9$ & $78.3(3)$ & $\mathrm{O}(47 \mathrm{~W})-\mathrm{Na}(16)-\mathrm{O}(87) \# 9$ & $87.2(3)$ \\
\hline $\mathrm{O}(47 \mathrm{~W}) \# 9-\mathrm{Na}(16)-\mathrm{O}(87) \# 9$ & $92.8(3)$ & $\mathrm{O}(87)-\mathrm{Na}(16)-\mathrm{O}(87) \# 9$ & 180.0 \\
\hline $\mathrm{O}(40 \mathrm{~W})-\mathrm{Na}(16)-\mathrm{U}(11) \# 9$ & $109.4(3)$ & $\mathrm{O}(40 \mathrm{~W}) \# 9-\mathrm{Na}(16)-\mathrm{U}(11) \# 9$ & $70.6(3)$ \\
\hline $\mathrm{O}(47 \mathrm{~W})-\mathrm{Na}(16)-\mathrm{U}(11) \# 9$ & $75.1(2)$ & $\mathrm{O}(47 \mathrm{~W}) \# 9-\mathrm{Na}(16)-\mathrm{U}(11) \# 9$ & $104.9(2)$ \\
\hline $\mathrm{O}(87)-\mathrm{Na}(16)-\mathrm{U}(11) \# 9$ & 165.39(19) & $\mathrm{O}(87) \# 9-\mathrm{Na}(16)-\mathrm{U}(11) \# 9$ & 14.61(19) \\
\hline $\mathrm{O}(40 \mathrm{~W})-\mathrm{Na}(16)-\mathrm{U}(11)$ & $70.6(3)$ & $\mathrm{O}(40 \mathrm{~W}) \# 9-\mathrm{Na}(16)-\mathrm{U}(11)$ & $109.4(3)$ \\
\hline $\mathrm{O}(47 \mathrm{~W})-\mathrm{Na}(16)-\mathrm{U}(11)$ & $104.9(2)$ & $\mathrm{O}(47 \mathrm{~W}) \# 9-\mathrm{Na}(16)-\mathrm{U}(11)$ & $75.1(2)$ \\
\hline $\mathrm{O}(87)-\mathrm{Na}(16)-\mathrm{U}(11)$ & 14.61(19) & $\mathrm{O}(87) \# 9-\mathrm{Na}(16)-\mathrm{U}(11)$ & 165.39(19) \\
\hline $\mathrm{U}(11) \# 9-\mathrm{Na}(16)-\mathrm{U}(11)$ & 180.0 & $\mathrm{O}(50 \mathrm{~W})-\mathrm{Na}(17)-\mathrm{O}(24 \mathrm{~W}) \# 6$ & $174.0(13)$ \\
\hline $\mathrm{O}(50 \mathrm{~W})-\mathrm{Na}(17)-\mathrm{O}(77 \mathrm{~W})$ & $90.4(8)$ & $\mathrm{O}(24 \mathrm{~W}) \# 6-\mathrm{Na}(17)-\mathrm{O}(77 \mathrm{~W})$ & $83.6(9)$ \\
\hline $\mathrm{O}(50 \mathrm{~W})-\mathrm{Na}(17)-\mathrm{O}(80)$ & $90.5(8)$ & $\mathrm{O}(24 \mathrm{~W}) \# 6-\mathrm{Na}(17)-\mathrm{O}(80)$ & $90.2(8)$ \\
\hline $\mathrm{O}(77 \mathrm{~W})-\mathrm{Na}(17)-\mathrm{O}(80)$ & $92.1(10)$ & $\mathrm{O}(50 \mathrm{~W})-\mathrm{Na}(17)-\mathrm{H}(41 \mathrm{~B})$ & $74.7(19)$ \\
\hline $\mathrm{O}(24 \mathrm{~W}) \# 6-\mathrm{Na}(17)-\mathrm{H}(41 \mathrm{~B})$ & 110.6(19) & $\mathrm{O}(77 \mathrm{~W})-\mathrm{Na}(17)-\mathrm{H}(41 \mathrm{~B})$ & $153.8(12)$ \\
\hline $\mathrm{O}(80)-\mathrm{Na}(17)-\mathrm{H}(41 \mathrm{~B})$ & $109.2(11)$ & $\mathrm{O}(50 \mathrm{~W})-\mathrm{Na}(17)-\mathrm{O}(49 \mathrm{~W})$ & $93.7(9)$ \\
\hline $\mathrm{O}(24 \mathrm{~W}) \# 6-\mathrm{Na}(17)-\mathrm{O}(49 \mathrm{~W})$ & $86.6(8)$ & $\mathrm{O}(77 \mathrm{~W})-\mathrm{Na}(17)-\mathrm{O}(49 \mathrm{~W})$ & $97.3(9)$ \\
\hline $\mathrm{O}(80)-\mathrm{Na}(17)-\mathrm{O}(49 \mathrm{~W})$ & 169.6(11) & $\mathrm{H}(41 \mathrm{~B})-\mathrm{Na}(17)-\mathrm{O}(49 \mathrm{~W})$ & $63.1(11)$ \\
\hline
\end{tabular}




\begin{tabular}{|c|c|c|c|}
\hline $\mathrm{O}(50 \mathrm{~W})-\mathrm{Na}(17)-\mathrm{O}(41 \mathrm{~W})$ & $81.3(9)$ & $\mathrm{O}(24 \mathrm{~W}) \# 6-\mathrm{Na}(17)-\mathrm{O}(41 \mathrm{~W})$ & $104.6(9)$ \\
\hline $\mathrm{O}(77 \mathrm{~W})-\mathrm{Na}(17)-\mathrm{O}(41 \mathrm{~W})$ & $171.7(10)$ & $\mathrm{O}(80)-\mathrm{Na}(17)-\mathrm{O}(41 \mathrm{~W})$ & $87.7(8)$ \\
\hline $\mathrm{H}(41 \mathrm{~B})-\mathrm{Na}(17)-\mathrm{O}(41 \mathrm{~W})$ & $22.6(5)$ & $\mathrm{O}(49 \mathrm{~W})-\mathrm{Na}(17)-\mathrm{O}(41 \mathrm{~W})$ & $83.6(9)$ \\
\hline $\mathrm{O}(50 \mathrm{~W})-\mathrm{Na}(17)-\mathrm{H}(50 \mathrm{~B})$ & $21.5(7)$ & $\mathrm{O}(24 \mathrm{~W}) \# 6-\mathrm{Na}(17)-\mathrm{H}(50 \mathrm{~B})$ & $156.0(14)$ \\
\hline $\mathrm{O}(77 \mathrm{~W})-\mathrm{Na}(17)-\mathrm{H}(50 \mathrm{~B})$ & $82.3(9)$ & $\mathrm{O}(80)-\mathrm{Na}(17)-\mathrm{H}(50 \mathrm{~B})$ & $71.1(8)$ \\
\hline $\mathrm{H}(41 \mathrm{~B})-\mathrm{Na}(17)-\mathrm{H}(50 \mathrm{~B})$ & $90(2)$ & $\mathrm{O}(49 \mathrm{~W})-\mathrm{Na}(17)-\mathrm{H}(50 \mathrm{~B})$ & $114.4(10)$ \\
\hline $\mathrm{O}(41 \mathrm{~W})-\mathrm{Na}(17)-\mathrm{H}(50 \mathrm{~B})$ & $89.8(9)$ & $\mathrm{O}(50 \mathrm{~W})-\mathrm{Na}(17)-\mathrm{Na}(8) \# 6$ & $135.0(10)$ \\
\hline $\mathrm{O}(24 \mathrm{~W}) \# 6-\mathrm{Na}(17)-\mathrm{Na}(8) \# 6$ & $45.6(5)$ & $\mathrm{O}(77 \mathrm{~W})-\mathrm{Na}(17)-\mathrm{Na}(8) \# 6$ & $88.3(8)$ \\
\hline $\mathrm{O}(80)-\mathrm{Na}(17)-\mathrm{Na}(8) \# 6$ & $44.6(5)$ & $\mathrm{H}(41 \mathrm{~B})-\mathrm{Na}(17)-\mathrm{Na}(8) \# 6$ & $117.6(12)$ \\
\hline $\mathrm{O}(49 \mathrm{~W})-\mathrm{Na}(17)-\mathrm{Na}(8) \# 6$ & $131.1(8)$ & $\mathrm{O}(41 \mathrm{~W})-\mathrm{Na}(17)-\mathrm{Na}(8) \# 6$ & $97.4(7)$ \\
\hline $\mathrm{H}(50 \mathrm{~B})-\mathrm{Na}(17)-\mathrm{Na}(8) \# 6$ & 114.6(10) & $\mathrm{O}(50 \mathrm{~W})-\mathrm{Na}(17)-\mathrm{P}(12)$ & $74.6(7)$ \\
\hline $\mathrm{O}(24 \mathrm{~W}) \# 6-\mathrm{Na}(17)-\mathrm{P}(12)$ & $104.4(9)$ & $\mathrm{O}(77 \mathrm{~W})-\mathrm{Na}(17)-\mathrm{P}(12)$ & $77.3(8)$ \\
\hline $\mathrm{O}(80)-\mathrm{Na}(17)-\mathrm{P}(12)$ & $22.0(3)$ & $H(41 B)-N a(17)-P(12)$ & $117.7(14)$ \\
\hline $\mathrm{O}(49 \mathrm{~W})-\mathrm{Na}(17)-\mathrm{P}(12)$ & $166.9(8)$ & $\mathrm{O}(41 \mathrm{~W})-\mathrm{Na}(17)-\mathrm{P}(12)$ & $100.1(7)$ \\
\hline $\mathrm{H}(50 \mathrm{~B})-\mathrm{Na}(17)-\mathrm{P}(12)$ & $53.4(7)$ & $\mathrm{Na}(8) \# 6-\mathrm{Na}(17)-\mathrm{P}(12)$ & $61.3(5)$ \\
\hline $\mathrm{O}(50 \mathrm{~W})-\mathrm{Na}(17)-\mathrm{Na}(8)$ & $87.6(7)$ & $\mathrm{O}(24 \mathrm{~W}) \# 6-\mathrm{Na}(17)-\mathrm{Na}(8)$ & $97.0(7)$ \\
\hline $\mathrm{O}(77 \mathrm{~W})-\mathrm{Na}(17)-\mathrm{Na}(8)$ & $135.5(10)$ & $\mathrm{O}(80)-\mathrm{Na}(17)-\mathrm{Na}(8)$ & $43.5(5)$ \\
\hline $\mathrm{H}(41 \mathrm{~B})-\mathrm{Na}(17)-\mathrm{Na}(8)$ & $66.5(8)$ & $\mathrm{O}(49 \mathrm{~W})-\mathrm{Na}(17)-\mathrm{Na}(8)$ & $127.2(10)$ \\
\hline $\mathrm{O}(41 \mathrm{~W})-\mathrm{Na}(17)-\mathrm{Na}(8)$ & $44.4(5)$ & $\mathrm{H}(50 \mathrm{~B})-\mathrm{Na}(17)-\mathrm{Na}(8)$ & $79.9(8)$ \\
\hline $\mathrm{Na}(8) \# 6-\mathrm{Na}(17)-\mathrm{Na}(8)$ & $63.4(5)$ & $\mathrm{P}(12)-\mathrm{Na}(17)-\mathrm{Na}(8)$ & $59.4(4)$ \\
\hline $\mathrm{O}(17 \mathrm{~W})-\mathrm{Na}(18)-\mathrm{O}(30 \mathrm{~W})$ & $169.0(14)$ & $\mathrm{O}(17 \mathrm{~W})-\mathrm{Na}(18)-\mathrm{O}(37 \mathrm{~W}) \# 10$ & $92.2(10)$ \\
\hline $\mathrm{O}(30 \mathrm{~W})-\mathrm{Na}(18)-\mathrm{O}(37 \mathrm{~W}) \# 10$ & $78.3(9)$ & $\mathrm{O}(17 \mathrm{~W})-\mathrm{Na}(18)-\mathrm{O}(38 \mathrm{~W})$ & $83.2(8)$ \\
\hline $\mathrm{O}(30 \mathrm{~W})-\mathrm{Na}(18)-\mathrm{O}(38 \mathrm{~W})$ & $104.6(11)$ & $\mathrm{O}(37 \mathrm{~W}) \# 10-\mathrm{Na}(18)-\mathrm{O}(38 \mathrm{~W})$ & $164.8(11)$ \\
\hline $\mathrm{O}(17 \mathrm{~W})-\mathrm{Na}(18)-\mathrm{H}(30 \mathrm{~B})$ & $168.8(15)$ & $\mathrm{O}(30 \mathrm{~W})-\mathrm{Na}(18)-\mathrm{H}(30 \mathrm{~B})$ & $21.9(8)$ \\
\hline $\mathrm{O}(37 \mathrm{~W}) \# 10-\mathrm{Na}(18)-\mathrm{H}(30 \mathrm{~B})$ & $98.9(11)$ & $\mathrm{O}(38 \mathrm{~W})-\mathrm{Na}(18)-\mathrm{H}(30 \mathrm{~B})$ & $86.5(11)$ \\
\hline $\mathrm{O}(17 \mathrm{~W})-\mathrm{Na}(18)-\mathrm{O}(29 \mathrm{~W})$ & $81.0(7)$ & $\mathrm{O}(30 \mathrm{~W})-\mathrm{Na}(18)-\mathrm{O}(29 \mathrm{~W})$ & $92.7(9)$ \\
\hline $\mathrm{O}(37 \mathrm{~W}) \# 10-\mathrm{Na}(18)-\mathrm{O}(29 \mathrm{~W})$ & $85.2(8)$ & $\mathrm{O}(38 \mathrm{~W})-\mathrm{Na}(18)-\mathrm{O}(29 \mathrm{~W})$ & $79.8(8)$ \\
\hline $\mathrm{H}(30 \mathrm{~B})-\mathrm{Na}(18)-\mathrm{O}(29 \mathrm{~W})$ & $101.8(11)$ & $\mathrm{O}(17 \mathrm{~W})-\mathrm{Na}(18)-\mathrm{Na}(15)$ & $49.0(6)$ \\
\hline $\mathrm{O}(30 \mathrm{~W})-\mathrm{Na}(18)-\mathrm{Na}(15)$ & $131.9(12)$ & $\mathrm{O}(37 \mathrm{~W}) \# 10-\mathrm{Na}(18)-\mathrm{Na}(15)$ & 119.9(9) \\
\hline $\mathrm{O}(38 \mathrm{~W})-\mathrm{Na}(18)-\mathrm{Na}(15)$ & $47.2(5)$ & $\mathrm{H}(30 \mathrm{~B})-\mathrm{Na}(18)-\mathrm{Na}(15)$ & $124.9(12)$ \\
\hline $\mathrm{O}(29 \mathrm{~W})-\mathrm{Na}(18)-\mathrm{Na}(15)$ & $50.5(5)$ & $\mathrm{O}(17 \mathrm{~W})-\mathrm{Na}(18)-\mathrm{Na}(13)$ & $128.6(9)$ \\
\hline $\mathrm{O}(30 \mathrm{~W})-\mathrm{Na}(18)-\mathrm{Na}(13)$ & $43.8(6)$ & $\mathrm{O}(37 \mathrm{~W}) \# 10-\mathrm{Na}(18)-\mathrm{Na}(13)$ & $75.2(7)$ \\
\hline $\mathrm{O}(38 \mathrm{~W})-\mathrm{Na}(18)-\mathrm{Na}(13)$ & $96.3(9)$ & $\mathrm{H}(30 \mathrm{~B})-\mathrm{Na}(18)-\mathrm{Na}(13)$ & $57.1(8)$ \\
\hline $\mathrm{O}(29 \mathrm{~W})-\mathrm{Na}(18)-\mathrm{Na}(13)$ & $49.0(5)$ & $\mathrm{Na}(15)-\mathrm{Na}(18)-\mathrm{Na}(13)$ & $94.7(7)$ \\
\hline $\mathrm{H}(20 \mathrm{~B}) \# 5-\mathrm{Na}(19)-\mathrm{O}(56 \mathrm{~W})$ & $146.8(14)$ & $\mathrm{H}(20 \mathrm{~B}) \# 5-\mathrm{Na}(19)-\mathrm{O}(6 \mathrm{~W})$ & $126.0(14)$ \\
\hline $\mathrm{O}(56 \mathrm{~W})-\mathrm{Na}(19)-\mathrm{O}(6 \mathrm{~W})$ & $82.3(10)$ & $\mathrm{H}(20 \mathrm{~B}) \# 5-\mathrm{Na}(19)-\mathrm{O}(17)$ & $66.3(13)$ \\
\hline $\mathrm{O}(56 \mathrm{~W})-\mathrm{Na}(19)-\mathrm{O}(17)$ & $98.3(9)$ & $\mathrm{O}(6 \mathrm{~W})-\mathrm{Na}(19)-\mathrm{O}(17)$ & $93.5(9)$ \\
\hline $\mathrm{H}(20 \mathrm{~B}) \# 5-\mathrm{Na}(19)-\mathrm{O}(20 \mathrm{~W}) \# 5$ & $22.9(5)$ & $\mathrm{O}(56 \mathrm{~W})-\mathrm{Na}(19)-\mathrm{O}(20 \mathrm{~W}) \# 5$ & $169.5(14)$ \\
\hline $\mathrm{O}(6 \mathrm{~W})-\mathrm{Na}(19)-\mathrm{O}(20 \mathrm{~W}) \# 5$ & $107.4(10)$ & $\mathrm{O}(17)-\mathrm{Na}(19)-\mathrm{O}(20 \mathrm{~W}) \# 5$ & $77.5(8)$ \\
\hline $\mathrm{H}(20 \mathrm{~B}) \# 5-\mathrm{Na}(19)-\mathrm{O}(21)$ & $45.5(8)$ & $\mathrm{O}(56 \mathrm{~W})-\mathrm{Na}(19)-\mathrm{O}(21)$ & $101.9(10)$ \\
\hline $\mathrm{O}(6 \mathrm{~W})-\mathrm{Na}(19)-\mathrm{O}(21)$ & $161.2(11)$ & $\mathrm{O}(17)-\mathrm{Na}(19)-\mathrm{O}(21)$ & $67.8(7)$ \\
\hline $\mathrm{O}(20 \mathrm{~W}) \# 5-\mathrm{Na}(19)-\mathrm{O}(21)$ & $67.6(7)$ & $\mathrm{H}(20 \mathrm{~B}) \# 5-\mathrm{Na}(19)-\mathrm{H}(56 \mathrm{~B})$ & $160.8(16)$ \\
\hline $\mathrm{O}(56 \mathrm{~W})-\mathrm{Na}(19)-\mathrm{H}(56 \mathrm{~B})$ & $21.2(5)$ & $\mathrm{O}(6 \mathrm{~W})-\mathrm{Na}(19)-\mathrm{H}(56 \mathrm{~B})$ & $73.2(10)$ \\
\hline $\mathrm{O}(17)-\mathrm{Na}(19)-\mathrm{H}(56 \mathrm{~B})$ & $117.3(10)$ & $\mathrm{O}(20 \mathrm{~W}) \# 5-\mathrm{Na}(19)-\mathrm{H}(56 \mathrm{~B})$ & $165.2(12)$ \\
\hline
\end{tabular}




\begin{tabular}{|c|c|c|c|}
\hline $\mathrm{O}(21)-\mathrm{Na}(19)-\mathrm{H}(56 \mathrm{~B})$ & $116.6(11)$ & $\mathrm{H}(20 \mathrm{~B}) \# 5-\mathrm{Na}(19)-\mathrm{O}(27 \mathrm{~W}) \# 5$ & $91.7(13)$ \\
\hline $\mathrm{O}(56 \mathrm{~W})-\mathrm{Na}(19)-\mathrm{O}(27 \mathrm{~W}) \# 5$ & $98.0(9)$ & $\mathrm{O}(6 \mathrm{~W})-\mathrm{Na}(19)-\mathrm{O}(27 \mathrm{~W}) \# 5$ & 104.1(10) \\
\hline $\mathrm{O}(17)-\mathrm{Na}(19)-\mathrm{O}(27 \mathrm{~W}) \# 5$ & $157.4(12)$ & $\mathrm{O}(20 \mathrm{~W}) \# 5-\mathrm{Na}(19)-\mathrm{O}(27 \mathrm{~W}) \# 5$ & $83.8(7)$ \\
\hline $\mathrm{O}(21)-\mathrm{Na}(19)-\mathrm{O}(27 \mathrm{~W}) \# 5$ & $93.5(8)$ & $\mathrm{H}(56 \mathrm{~B})-\mathrm{Na}(19)-\mathrm{O}(27 \mathrm{~W}) \# 5$ & $81.8(9)$ \\
\hline $\mathrm{H}(20 \mathrm{~B}) \# 5-\mathrm{Na}(19)-\mathrm{P}(11)$ & $56.1(11)$ & $\mathrm{O}(56 \mathrm{~W})-\mathrm{Na}(19)-\mathrm{P}(11)$ & $98.7(9)$ \\
\hline $\mathrm{O}(6 \mathrm{~W})-\mathrm{Na}(19)-\mathrm{P}(11)$ & $171.7(10)$ & $\mathrm{O}(17)-\mathrm{Na}(19)-\mathrm{P}(11)$ & $94.4(9)$ \\
\hline $\mathrm{O}(20 \mathrm{~W}) \# 5-\mathrm{Na}(19)-\mathrm{P}(11)$ & $72.3(7)$ & $\mathrm{O}(21)-\mathrm{Na}(19)-\mathrm{P}(11)$ & $26.6(3)$ \\
\hline $\mathrm{H}(56 \mathrm{~B})-\mathrm{Na}(19)-\mathrm{P}(11)$ & $105.0(10)$ & $\mathrm{O}(27 \mathrm{~W}) \# 5-\mathrm{Na}(19)-\mathrm{P}(11)$ & $67.6(6)$ \\
\hline $\mathrm{H}(20 \mathrm{~B}) \# 5-\mathrm{Na}(19)-\mathrm{Na}(4) \# 5$ & $52.1(11)$ & $\mathrm{O}(56 \mathrm{~W})-\mathrm{Na}(19)-\mathrm{Na}(4) \# 5$ & $138.6(10)$ \\
\hline $\mathrm{O}(6 \mathrm{~W})-\mathrm{Na}(19)-\mathrm{Na}(4) \# 5$ & $113.4(9)$ & $\mathrm{O}(17)-\mathrm{Na}(19)-\mathrm{Na}(4) \# 5$ & $117.6(9)$ \\
\hline $\mathrm{O}(20 \mathrm{~W}) \# 5-\mathrm{Na}(19)-\mathrm{Na}(4) \# 5$ & $41.7(4)$ & $\mathrm{O}(21)-\mathrm{Na}(19)-\mathrm{Na}(4) \# 5$ & $75.6(6)$ \\
\hline $\mathrm{H}(56 \mathrm{~B})-\mathrm{Na}(19)-\mathrm{Na}(4) \mathrm{\# 5}$ & $124.0(10)$ & $\mathrm{O}(27 \mathrm{~W}) \# 5-\mathrm{Na}(19)-\mathrm{Na}(4) \# 5$ & $42.2(4)$ \\
\hline $\mathrm{P}(11)-\mathrm{Na}(19)-\mathrm{Na}(4) \# 5$ & $60.6(5)$ & $\mathrm{H}(20 \mathrm{~B}) \# 5-\mathrm{Na}(19)-\mathrm{U}(3)$ & $66.4(9)$ \\
\hline $\mathrm{O}(56 \mathrm{~W})-\mathrm{Na}(19)-\mathrm{U}(3)$ & $83.3(8)$ & $\mathrm{O}(6 \mathrm{~W})-\mathrm{Na}(19)-\mathrm{U}(3)$ & $125.6(8)$ \\
\hline $\mathrm{O}(17)-\mathrm{Na}(19)-\mathrm{U}(3)$ & $37.9(5)$ & $\mathrm{O}(20 \mathrm{~W}) \# 5-\mathrm{Na}(19)-\mathrm{U}(3)$ & $87.6(8)$ \\
\hline $\mathrm{O}(21)-\mathrm{Na}(19)-\mathrm{U}(3)$ & $38.6(4)$ & $\mathrm{H}(56 \mathrm{~B})-\mathrm{Na}(19)-\mathrm{U}(3)$ & $104.2(8)$ \\
\hline $\mathrm{O}(27 \mathrm{~W}) \# 5-\mathrm{Na}(19)-\mathrm{U}(3)$ & $129.7(9)$ & $\mathrm{P}(11)-\mathrm{Na}(19)-\mathrm{U}(3)$ & $62.6(5)$ \\
\hline $\mathrm{Na}(4) \# 5-\mathrm{Na}(19)-\mathrm{U}(3)$ & $111.9(7)$ & $\mathrm{H}(20 \mathrm{~B}) \# 5-\mathrm{Na}(19)-\mathrm{Na}(11)$ & $130.7(14)$ \\
\hline $\mathrm{O}(56 \mathrm{~W})-\mathrm{Na}(19)-\mathrm{Na}(11)$ & $63.2(7)$ & $\mathrm{O}(6 \mathrm{~W})-\mathrm{Na}(19)-\mathrm{Na}(11)$ & $31.8(4)$ \\
\hline $\mathrm{O}(17)-\mathrm{Na}(19)-\mathrm{Na}(11)$ & $72.5(6)$ & $\mathrm{O}(20 \mathrm{~W}) \# 5-\mathrm{Na}(19)-\mathrm{Na}(11)$ & $123.3(9)$ \\
\hline $\mathrm{O}(21)-\mathrm{Na}(19)-\mathrm{Na}(11)$ & $134.9(8)$ & $\mathrm{H}(56 \mathrm{~B})-\mathrm{Na}(19)-\mathrm{Na}(11)$ & $65.0(9)$ \\
\hline $\mathrm{O}(27 \mathrm{~W}) \# 5-\mathrm{Na}(19)-\mathrm{Na}(11)$ & $129.2(9)$ & $\mathrm{P}(11)-\mathrm{Na}(19)-\mathrm{Na}(11)$ & $154.8(8)$ \\
\hline $\mathrm{Na}(4) \# 5-\mathrm{Na}(19)-\mathrm{Na}(11)$ & $144.5(8)$ & $\mathrm{U}(3)-\mathrm{Na}(19)-\mathrm{Na}(11)$ & $96.3(5)$ \\
\hline $\mathrm{Na}(21)-\mathrm{Na}(20)-\mathrm{O}(73 \mathrm{~W})$ & $139(4)$ & $\mathrm{Na}(21)-\mathrm{Na}(20)-\mathrm{O}(70 \mathrm{~W})$ & $39(3)$ \\
\hline $\mathrm{O}(73 \mathrm{~W})-\mathrm{Na}(20)-\mathrm{O}(70 \mathrm{~W})$ & $99.4(15)$ & $\mathrm{Na}(21)-\mathrm{Na}(20)-\mathrm{O}(60 \mathrm{~W})$ & $61(3)$ \\
\hline $\mathrm{O}(73 \mathrm{~W})-\mathrm{Na}(20)-\mathrm{O}(60 \mathrm{~W})$ & $156.7(16)$ & $\mathrm{O}(70 \mathrm{~W})-\mathrm{Na}(20)-\mathrm{O}(60 \mathrm{~W})$ & $99.3(14)$ \\
\hline $\mathrm{Na}(21)-\mathrm{Na}(20)-\mathrm{O}(43 \mathrm{~W})$ & $134(4)$ & $\mathrm{O}(73 \mathrm{~W})-\mathrm{Na}(20)-\mathrm{O}(43 \mathrm{~W})$ & $85.3(12)$ \\
\hline $\mathrm{O}(70 \mathrm{~W})-\mathrm{Na}(20)-\mathrm{O}(43 \mathrm{~W})$ & $162.6(14)$ & $\mathrm{O}(60 \mathrm{~W})-\mathrm{Na}(20)-\mathrm{O}(43 \mathrm{~W})$ & $81.0(11)$ \\
\hline $\mathrm{Na}(21)-\mathrm{Na}(20)-\mathrm{O}(2 \mathrm{I})$ & $85(3)$ & $\mathrm{O}(73 \mathrm{~W})-\mathrm{Na}(20)-\mathrm{O}(2 \mathrm{I})$ & $92.2(11)$ \\
\hline $\mathrm{O}(70 \mathrm{~W})-\mathrm{Na}(20)-\mathrm{O}(2 \mathrm{I})$ & $83.9(11)$ & $\mathrm{O}(60 \mathrm{~W})-\mathrm{Na}(20)-\mathrm{O}(2 \mathrm{I})$ & $103.5(11)$ \\
\hline $\mathrm{O}(43 \mathrm{~W})-\mathrm{Na}(20)-\mathrm{O}(2 \mathrm{I})$ & $79.2(9)$ & $\mathrm{Na}(21)-\mathrm{Na}(20)-\mathrm{H}(73 \mathrm{~B})$ & $143(4)$ \\
\hline $\mathrm{O}(73 \mathrm{~W})-\mathrm{Na}(20)-\mathrm{H}(73 \mathrm{~B})$ & $20.8(13)$ & $\mathrm{O}(70 \mathrm{~W})-\mathrm{Na}(20)-\mathrm{H}(73 \mathrm{~B})$ & $107(3)$ \\
\hline $\mathrm{O}(60 \mathrm{~W})-\mathrm{Na}(20)-\mathrm{H}(73 \mathrm{~B})$ & 137.3(19) & $\mathrm{O}(43 \mathrm{~W})-\mathrm{Na}(20)-\mathrm{H}(73 \mathrm{~B})$ & $83(3)$ \\
\hline $\mathrm{O}(2 \mathrm{I})-\mathrm{Na}(20)-\mathrm{H}(73 \mathrm{~B})$ & $111.7(19)$ & $\mathrm{Na}(21)-\mathrm{Na}(20)-\mathrm{Na}(5)$ & $102(3)$ \\
\hline $\mathrm{O}(73 \mathrm{~W})-\mathrm{Na}(20)-\mathrm{Na}(5)$ & $102.4(11)$ & $\mathrm{O}(70 \mathrm{~W})-\mathrm{Na}(20)-\mathrm{Na}(5)$ & $120.1(12)$ \\
\hline $\mathrm{O}(60 \mathrm{~W})-\mathrm{Na}(20)-\mathrm{Na}(5)$ & $79.9(9)$ & $\mathrm{O}(43 \mathrm{~W})-\mathrm{Na}(20)-\mathrm{Na}(5)$ & $42.7(6)$ \\
\hline $\mathrm{O}(2 \mathrm{I})-\mathrm{Na}(20)-\mathrm{Na}(5)$ & $40.5(5)$ & $\mathrm{H}(73 \mathrm{~B})-\mathrm{Na}(20)-\mathrm{Na}(5)$ & $112(3)$ \\
\hline $\mathrm{Na}(20)-\mathrm{Na}(21)-\mathrm{O}(70 \mathrm{~W})$ & $112(5)$ & $\mathrm{Na}(20)-\mathrm{Na}(21)-\mathrm{O}(60 \mathrm{~W})$ & $87(4)$ \\
\hline $\mathrm{O}(70 \mathrm{~W})-\mathrm{Na}(21)-\mathrm{O}(60 \mathrm{~W})$ & $156(4)$ & $\mathrm{Na}(20)-\mathrm{Na}(21)-\mathrm{H}(70 \mathrm{~A})$ & $101(4)$ \\
\hline $\mathrm{O}(70 \mathrm{~W})-\mathrm{Na}(21)-\mathrm{H}(70 \mathrm{~A})$ & $25.0(15)$ & $\mathrm{O}(60 \mathrm{~W})-\mathrm{Na}(21)-\mathrm{H}(70 \mathrm{~A})$ & $168(4)$ \\
\hline $\mathrm{Na}(20)-\mathrm{Na}(21)-\mathrm{H}(60 \mathrm{~A})$ & $105(4)$ & $\mathrm{O}(70 \mathrm{~W})-\mathrm{Na}(21)-\mathrm{H}(60 \mathrm{~A})$ & 132(4) \\
\hline $\mathrm{O}(60 \mathrm{~W})-\mathrm{Na}(21)-\mathrm{H}(60 \mathrm{~A})$ & $24.5(10)$ & $\mathrm{H}(70 \mathrm{~A})-\mathrm{Na}(21)-\mathrm{H}(60 \mathrm{~A})$ & $153(4)$ \\
\hline $\mathrm{Na}(20)-\mathrm{Na}(21)-\mathrm{H}(70 \mathrm{~B})$ & $109(5)$ & $\mathrm{O}(70 \mathrm{~W})-\mathrm{Na}(21)-\mathrm{H}(70 \mathrm{~B})$ & $15(3)$ \\
\hline $\mathrm{O}(60 \mathrm{~W})-\mathrm{Na}(21)-\mathrm{H}(70 \mathrm{~B})$ & $146(5)$ & $\mathrm{H}(70 \mathrm{~A})-\mathrm{Na}(21)-\mathrm{H}(70 \mathrm{~B})$ & $39(3)$ \\
\hline
\end{tabular}




\begin{tabular}{|c|c|c|c|}
\hline $\mathrm{H}(60 \mathrm{~A})-\mathrm{Na}(21)-\mathrm{H}(70 \mathrm{~B})$ & $122(4)$ & $\mathrm{Na}(20)-\mathrm{Na}(21)-\mathrm{O}(2 \mathrm{I})$ & $67(3)$ \\
\hline $\mathrm{O}(70 \mathrm{~W})-\mathrm{Na}(21)-\mathrm{O}(2 \mathrm{I})$ & $95(3)$ & $\mathrm{O}(60 \mathrm{~W})-\mathrm{Na}(21)-\mathrm{O}(2 \mathrm{I})$ & $106(3)$ \\
\hline $\mathrm{H}(70 \mathrm{~A})-\mathrm{Na}(21)-\mathrm{O}(2 \mathrm{I})$ & $70(2)$ & $\mathrm{H}(60 \mathrm{~A})-\mathrm{Na}(21)-\mathrm{O}(2 \mathrm{I})$ & $128(3)$ \\
\hline $\mathrm{H}(70 \mathrm{~B})-\mathrm{Na}(21)-\mathrm{O}(2 \mathrm{I})$ & $108(4)$ & $\mathrm{Na}(20)-\mathrm{Na}(21)-\mathrm{Na}(5)$ & $60(3)$ \\
\hline $\mathrm{O}(70 \mathrm{~W})-\mathrm{Na}(21)-\mathrm{Na}(5)$ & $129(3)$ & $\mathrm{O}(60 \mathrm{~W})-\mathrm{Na}(21)-\mathrm{Na}(5)$ & $73(2)$ \\
\hline $\mathrm{H}(70 \mathrm{~A})-\mathrm{Na}(21)-\mathrm{Na}(5)$ & $104(2)$ & $\mathrm{H}(60 \mathrm{~A})-\mathrm{Na}(21)-\mathrm{Na}(5)$ & $96(2)$ \\
\hline $\mathrm{H}(70 \mathrm{~B})-\mathrm{Na}(21)-\mathrm{Na}(5)$ & $142(4)$ & $\mathrm{O}(2 \mathrm{I})-\mathrm{Na}(21)-\mathrm{Na}(5)$ & $33.8(9)$ \\
\hline $\mathrm{O}(9)-\mathrm{P}(1)-\mathrm{O}(70)$ & $113.8(7)$ & $\mathrm{O}(9)-\mathrm{P}(1)-\mathrm{O}(44)$ & $111.8(7)$ \\
\hline $\mathrm{O}(70)-\mathrm{P}(1)-\mathrm{O}(44)$ & $112.7(7)$ & $\mathrm{O}(9)-\mathrm{P}(1)-\mathrm{O}(71)$ & $106.6(6)$ \\
\hline $\mathrm{O}(70)-\mathrm{P}(1)-\mathrm{O}(71)$ & $105.5(6)$ & $\mathrm{O}(44)-\mathrm{P}(1)-\mathrm{O}(71)$ & $105.6(6)$ \\
\hline $\mathrm{O}(72)-\mathrm{P}(2)-\mathrm{O}(57)$ & $115.9(7)$ & $\mathrm{O}(72)-\mathrm{P}(2)-\mathrm{O}(27)$ & $113.6(7)$ \\
\hline $\mathrm{O}(57)-\mathrm{P}(2)-\mathrm{O}(27)$ & $110.6(7)$ & $\mathrm{O}(72)-\mathrm{P}(2)-\mathrm{O}(73)$ & $105.3(7)$ \\
\hline $\mathrm{O}(57)-\mathrm{P}(2)-\mathrm{O}(73)$ & $105.3(6)$ & $\mathrm{O}(27)-\mathrm{P}(2)-\mathrm{O}(73)$ & $105.0(6)$ \\
\hline $\mathrm{O}(72)-\mathrm{P}(2)-\mathrm{Na}(15)$ & $32.4(4)$ & $\mathrm{O}(57)-\mathrm{P}(2)-\mathrm{Na}(15)$ & $89.8(5)$ \\
\hline $\mathrm{O}(27)-\mathrm{P}(2)-\mathrm{Na}(15)$ & $145.3(5)$ & $\mathrm{O}(73)-\mathrm{P}(2)-\mathrm{Na}(15)$ & $95.7(5)$ \\
\hline$O(72)-P(2)-K(2)$ & $115.1(6)$ & $\mathrm{O}(57)-\mathrm{P}(2)-\mathrm{K}(2)$ & $45.5(4)$ \\
\hline$O(27)-P(2)-K(2)$ & $70.9(5)$ & $\mathrm{O}(73)-\mathrm{P}(2)-\mathrm{K}(2)$ & $137.6(5)$ \\
\hline $\mathrm{Na}(15)-\mathrm{P}(2)-\mathrm{K}(2)$ & $111.2(4)$ & $\mathrm{O}(88)-\mathrm{P}(3)-\mathrm{O}(62)$ & $114.1(8)$ \\
\hline $\mathrm{O}(88)-\mathrm{P}(3)-\mathrm{O}(50)$ & $113.0(8)$ & $\mathrm{O}(62)-\mathrm{P}(3)-\mathrm{O}(50)$ & $110.5(8)$ \\
\hline $\mathrm{O}(88)-\mathrm{P}(3)-\mathrm{O}(84)$ & $105.5(8)$ & $\mathrm{O}(62)-\mathrm{P}(3)-\mathrm{O}(84)$ & $106.2(7)$ \\
\hline $\mathrm{O}(50)-\mathrm{P}(3)-\mathrm{O}(84)$ & $107.0(7)$ & $\mathrm{O}(75)-\mathrm{P}(4)-\mathrm{O}(6)$ & $114.2(7)$ \\
\hline $\mathrm{O}(75)-\mathrm{P}(4)-\mathrm{O}(20)$ & $112.7(7)$ & $\mathrm{O}(6)-\mathrm{P}(4)-\mathrm{O}(20)$ & $112.4(7)$ \\
\hline $\mathrm{O}(75)-\mathrm{P}(4)-\mathrm{O}(74) \# 1$ & $104.5(6)$ & $\mathrm{O}(6)-\mathrm{P}(4)-\mathrm{O}(74) \# 1$ & $106.3(6)$ \\
\hline $\mathrm{O}(20)-\mathrm{P}(4)-\mathrm{O}(74) \# 1$ & $105.8(6)$ & $\mathrm{O}(89)-\mathrm{P}(5)-\mathrm{O}(32)$ & $115.1(7)$ \\
\hline $\mathrm{O}(89)-\mathrm{P}(5)-\mathrm{O}(51)$ & $114.3(8)$ & $\mathrm{O}(32)-\mathrm{P}(5)-\mathrm{O}(51)$ & $110.0(7)$ \\
\hline $\mathrm{O}(89)-\mathrm{P}(5)-\mathrm{O}(82)$ & $104.5(6)$ & $\mathrm{O}(32)-\mathrm{P}(5)-\mathrm{O}(82)$ & $106.5(7)$ \\
\hline $\mathrm{O}(51)-\mathrm{P}(5)-\mathrm{O}(82)$ & $105.6(6)$ & $\mathrm{O}(89)-\mathrm{P}(5)-\mathrm{Na}(12)$ & $43.8(5)$ \\
\hline $\mathrm{O}(32)-\mathrm{P}(5)-\mathrm{Na}(12)$ & $120.2(5)$ & $\mathrm{O}(51)-\mathrm{P}(5)-\mathrm{Na}(12)$ & $129.8(6)$ \\
\hline $\mathrm{O}(82)-\mathrm{P}(5)-\mathrm{Na}(12)$ & $61.1(4)$ & $\mathrm{O}(90)-\mathrm{P}(6)-\mathrm{O}(86)$ & $115.2(9)$ \\
\hline $\mathrm{O}(90)-\mathrm{P}(6)-\mathrm{O}(65)$ & $111.8(8)$ & $\mathrm{O}(86)-\mathrm{P}(6)-\mathrm{O}(65)$ & $108.4(7)$ \\
\hline $\mathrm{O}(90)-\mathrm{P}(6)-\mathrm{O}(84)$ & $107.9(7)$ & $\mathrm{O}(86)-\mathrm{P}(6)-\mathrm{O}(84)$ & $107.0(7)$ \\
\hline $\mathrm{O}(65)-\mathrm{P}(6)-\mathrm{O}(84)$ & $106.0(7)$ & $\mathrm{O}(76)-\mathrm{P}(7)-\mathrm{O}(11)$ & $114.7(7)$ \\
\hline $\mathrm{O}(76)-\mathrm{P}(7)-\mathrm{O}(23)$ & $113.0(7)$ & $\mathrm{O}(11)-\mathrm{P}(7)-\mathrm{O}(23)$ & $109.8(7)$ \\
\hline $\mathrm{O}(76)-\mathrm{P}(7)-\mathrm{O}(71)$ & $106.1(6)$ & $\mathrm{O}(11)-\mathrm{P}(7)-\mathrm{O}(71)$ & $106.6(6)$ \\
\hline $\mathrm{O}(23)-\mathrm{P}(7)-\mathrm{O}(71)$ & $105.9(6)$ & $\mathrm{O}(76)-\mathrm{P}(7)-\mathrm{K}(1)$ & $118.9(5)$ \\
\hline$O(11)-P(7)-K(1)$ & $51.3(5)$ & $\mathrm{O}(23)-\mathrm{P}(7)-\mathrm{K}(1)$ & $61.5(5)$ \\
\hline$O(71)-P(7)-K(1)$ & $134.7(5)$ & $\mathrm{O}(81)-\mathrm{P}(8)-\mathrm{O}(28)$ & $112.2(7)$ \\
\hline $\mathrm{O}(81)-\mathrm{P}(8)-\mathrm{O}(58)$ & $114.6(7)$ & $\mathrm{O}(28)-\mathrm{P}(8)-\mathrm{O}(58)$ & $111.9(7)$ \\
\hline $\mathrm{O}(81)-\mathrm{P}(8)-\mathrm{O}(73)$ & $104.8(7)$ & $\mathrm{O}(28)-\mathrm{P}(8)-\mathrm{O}(73)$ & $106.9(6)$ \\
\hline $\mathrm{O}(58)-\mathrm{P}(8)-\mathrm{O}(73)$ & $105.8(6)$ & $\mathrm{O}(78)-\mathrm{P}(9)-\mathrm{O}(67)$ & $114.3(7)$ \\
\hline $\mathrm{O}(78)-\mathrm{P}(9)-\mathrm{O}(34)$ & $112.6(7)$ & $\mathrm{O}(67)-\mathrm{P}(9)-\mathrm{O}(34)$ & $111.1(7)$ \\
\hline $\mathrm{O}(78)-\mathrm{P}(9)-\mathrm{O}(79)$ & $105.5(6)$ & $\mathrm{O}(67)-\mathrm{P}(9)-\mathrm{O}(79)$ & 106.7(6) \\
\hline $\mathrm{O}(34)-\mathrm{P}(9)-\mathrm{O}(79)$ & $105.9(7)$ & $\mathrm{O}(83)-\mathrm{P}(10)-\mathrm{O}(31)$ & $112.5(7)$ \\
\hline $\mathrm{O}(83)-\mathrm{P}(10)-\mathrm{O}(53)$ & $112.4(7)$ & $\mathrm{O}(31)-\mathrm{P}(10)-\mathrm{O}(53)$ & $112.4(6)$ \\
\hline
\end{tabular}




\begin{tabular}{|c|c|c|c|}
\hline $\mathrm{O}(83)-\mathrm{P}(10)-\mathrm{O}(82)$ & $105.8(6)$ & $\mathrm{O}(31)-\mathrm{P}(10)-\mathrm{O}(82)$ & $106.7(6)$ \\
\hline $\mathrm{O}(53)-\mathrm{P}(10)-\mathrm{O}(82)$ & $106.3(7)$ & $\mathrm{O}(77)-\mathrm{P}(11)-\mathrm{O}(21)$ & $113.8(7)$ \\
\hline $\mathrm{O}(77)-\mathrm{P}(11)-\mathrm{O}(7)$ & $114.8(7)$ & $\mathrm{O}(21)-\mathrm{P}(11)-\mathrm{O}(7)$ & $111.2(7)$ \\
\hline $\mathrm{O}(77)-\mathrm{P}(11)-\mathrm{O}(74)$ & $105.1(6)$ & $\mathrm{O}(21)-\mathrm{P}(11)-\mathrm{O}(74)$ & $105.7(6)$ \\
\hline $\mathrm{O}(7)-\mathrm{P}(11)-\mathrm{O}(74)$ & $105.3(6)$ & $\mathrm{O}(77)-\mathrm{P}(11)-\mathrm{Na}(19)$ & $99.5(6)$ \\
\hline $\mathrm{O}(21)-\mathrm{P}(11)-\mathrm{Na}(19)$ & $50.2(6)$ & $\mathrm{O}(7)-\mathrm{P}(11)-\mathrm{Na}(19)$ & $76.3(6)$ \\
\hline $\mathrm{O}(74)-\mathrm{P}(11)-\mathrm{Na}(19)$ & $151.6(6)$ & $\mathrm{O}(77)-\mathrm{P}(11)-\mathrm{Na}(14)$ & $37.3(5)$ \\
\hline $\mathrm{O}(21)-\mathrm{P}(11)-\mathrm{Na}(14)$ & $87.3(5)$ & $\mathrm{O}(7)-\mathrm{P}(11)-\mathrm{Na}(14)$ & $152.0(6)$ \\
\hline $\mathrm{O}(74)-\mathrm{P}(11)-\mathrm{Na}(14)$ & $88.6(5)$ & $\mathrm{Na}(19)-\mathrm{P}(11)-\mathrm{Na}(14)$ & $102.9(6)$ \\
\hline $\mathrm{O}(80)-\mathrm{P}(12)-\mathrm{O}(68)$ & $115.1(7)$ & $\mathrm{O}(80)-\mathrm{P}(12)-\mathrm{O}(37)$ & $112.3(7)$ \\
\hline $\mathrm{O}(68)-\mathrm{P}(12)-\mathrm{O}(37)$ & $111.3(7)$ & $\mathrm{O}(80)-\mathrm{P}(12)-\mathrm{O}(79)$ & $106.7(6)$ \\
\hline $\mathrm{O}(68)-\mathrm{P}(12)-\mathrm{O}(79)$ & $105.6(7)$ & $\mathrm{O}(37)-\mathrm{P}(12)-\mathrm{O}(79)$ & $105.1(6)$ \\
\hline $\mathrm{O}(80)-\mathrm{P}(12)-\mathrm{Na}(17)$ & $37.3(5)$ & $\mathrm{O}(68)-\mathrm{P}(12)-\mathrm{Na}(17)$ & $107.3(7)$ \\
\hline $\mathrm{O}(37)-\mathrm{P}(12)-\mathrm{Na}(17)$ & $83.7(6)$ & $\mathrm{O}(79)-\mathrm{P}(12)-\mathrm{Na}(17)$ & $139.8(6)$ \\
\hline $\mathrm{O}(80)-\mathrm{P}(12)-\mathrm{Na}(8)$ & $37.2(4)$ & $\mathrm{O}(68)-\mathrm{P}(12)-\mathrm{Na}(8)$ & $148.1(6)$ \\
\hline $\mathrm{O}(37)-\mathrm{P}(12)-\mathrm{Na}(8)$ & $97.6(5)$ & $\mathrm{O}(79)-\mathrm{P}(12)-\mathrm{Na}(8)$ & $78.4(5)$ \\
\hline $\mathrm{Na}(17)-\mathrm{P}(12)-\mathrm{Na}(8)$ & $61.5(5)$ & $\mathrm{O}(80)-\mathrm{P}(12)-\mathrm{Na}(8) \# 6$ & $33.7(4)$ \\
\hline $\mathrm{O}(68)-\mathrm{P}(12)-\mathrm{Na}(8) \# 6$ & $85.2(5)$ & $\mathrm{O}(37)-\mathrm{P}(12)-\mathrm{Na}(8) \# 6$ & $142.3(6)$ \\
\hline $\mathrm{O}(79)-\mathrm{P}(12)-\mathrm{Na}(8) \# 6$ & $102.4(5)$ & $\mathrm{Na}(17)-\mathrm{P}(12)-\mathrm{Na}(8) \# 6$ & $58.7(4)$ \\
\hline $\mathrm{Na}(8)-\mathrm{P}(12)-\mathrm{Na}(8) \# 6$ & $63.3(4)$ & $\mathrm{O}(80)-\mathrm{P}(12)-\mathrm{K}(3)$ & $115.3(6)$ \\
\hline $\mathrm{O}(68)-\mathrm{P}(12)-\mathrm{K}(3)$ & $46.5(5)$ & $\mathrm{O}(37)-\mathrm{P}(12)-\mathrm{K}(3)$ & $69.5(5)$ \\
\hline $\mathrm{O}(79)-\mathrm{P}(12)-\mathrm{K}(3)$ & $136.6(6)$ & $\mathrm{Na}(17)-\mathrm{P}(12)-\mathrm{K}(3)$ & $83.5(5)$ \\
\hline $\mathrm{Na}(8)-\mathrm{P}(12)-\mathrm{K}(3)$ & $144.2(5)$ & $\mathrm{Na}(8) \# 6-\mathrm{P}(12)-\mathrm{K}(3)$ & $106.1(4)$ \\
\hline $\mathrm{O}(8)-\mathrm{O}(1)-\mathrm{U}(1)$ & $75.6(5)$ & $\mathrm{O}(8)-\mathrm{O}(1)-\mathrm{U}(6)$ & $74.2(5)$ \\
\hline$U(1)-O(1)-U(6)$ & $127.9(4)$ & $O(4)-O(2)-U(1)$ & $70.7(4)$ \\
\hline$O(4)-O(2)-U(11)$ & $69.5(4)$ & $U(1)-O(2)-U(11)$ & $122.8(4)$ \\
\hline $\mathrm{U}(1)-\mathrm{O}(3)-\mathrm{Na}(1)$ & $141.8(6)$ & $\mathrm{O}(2)-\mathrm{O}(4)-\mathrm{U}(11)$ & $74.5(4)$ \\
\hline $\mathrm{O}(2)-\mathrm{O}(4)-\mathrm{U}(1)$ & $73.5(4)$ & $U(11)-O(4)-U(1)$ & $127.7(4)$ \\
\hline$P(4)-O(6)-U(1)$ & $142.6(6)$ & $\mathrm{P}(11)-\mathrm{O}(7)-\mathrm{U}(1) \# 1$ & $137.8(6)$ \\
\hline $\mathrm{O}(1)-\mathrm{O}(8)-\mathrm{U}(6)$ & $69.9(5)$ & $\mathrm{O}(1)-\mathrm{O}(8)-\mathrm{U}(1)$ & $68.7(5)$ \\
\hline$U(6)-O(8)-U(1)$ & $121.1(4)$ & $P(1)-O(9)-U(2)$ & $142.7(5)$ \\
\hline $\mathrm{O}(42)-\mathrm{O}(10)-\mathrm{U}(9) \# 1$ & $73.6(4)$ & $O(42)-O(10)-U(2)$ & $74.1(4)$ \\
\hline$U(9) \# 1-O(10)-U(2)$ & $128.3(4)$ & $\mathrm{P}(7)-\mathrm{O}(11)-\mathrm{U}(2)$ & $137.6(5)$ \\
\hline $\mathrm{P}(7)-\mathrm{O}(11)-\mathrm{K}(1)$ & $105.3(6)$ & $U(2)-O(11)-K(1)$ & $91.6(4)$ \\
\hline $\mathrm{U}(2)-\mathrm{O}(12)-\mathrm{Na}(11)$ & $137.4(5)$ & $U(2)-O(12)-K(1)$ & $108.4(4)$ \\
\hline $\mathrm{Na}(11)-\mathrm{O}(12)-\mathrm{K}(1)$ & $103.2(5)$ & $O(15)-O(13)-U(2)$ & $74.3(5)$ \\
\hline $\mathrm{O}(15)-\mathrm{O}(13)-\mathrm{U}(8)$ & $75.2(4)$ & $U(2)-O(13)-U(8)$ & $125.1(4)$ \\
\hline$U(2)-O(14)-\mathrm{Na}(2)$ & $148.2(6)$ & $O(13)-O(15)-U(2)$ & $69.4(4)$ \\
\hline $\mathrm{O}(13)-\mathrm{O}(15)-\mathrm{U}(8)$ & $69.0(4)$ & $U(2)-O(15)-U(8)$ & $118.5(3)$ \\
\hline $\mathrm{O}(13)-\mathrm{O}(15)-\mathrm{K}(1)$ & $128.9(6)$ & $U(2)-O(15)-K(1)$ & $83.7(4)$ \\
\hline $\mathrm{U}(8)-\mathrm{O}(15)-\mathrm{K}(1)$ & $89.1(4)$ & $O(19)-O(16)-U(3)$ & $74.8(4)$ \\
\hline $\mathrm{O}(19)-\mathrm{O}(16)-\mathrm{U}(5)$ & $75.5(5)$ & $U(3)-O(16)-U(5)$ & $132.1(4)$ \\
\hline $\mathrm{O}(18)-\mathrm{O}(17)-\mathrm{Na}(19)$ & $138.4(8)$ & $\mathrm{O}(18)-\mathrm{O}(17)-\mathrm{U}(4)$ & $68.4(4)$ \\
\hline $\mathrm{Na}(19)-\mathrm{O}(17)-\mathrm{U}(4)$ & $131.1(7)$ & $\mathrm{O}(18)-\mathrm{O}(17)-\mathrm{U}(3)$ & $68.2(4)$ \\
\hline
\end{tabular}




\begin{tabular}{|c|c|c|c|}
\hline $\mathrm{Na}(19)-\mathrm{O}(17)-\mathrm{U}(3)$ & $105.6(7)$ & $U(4)-O(17)-U(3)$ & $123.3(3)$ \\
\hline $\mathrm{O}(17)-\mathrm{O}(18)-\mathrm{U}(4)$ & $75.2(4)$ & $\mathrm{O}(17)-\mathrm{O}(18)-\mathrm{U}(3)$ & $75.6(4)$ \\
\hline$U(4)-O(18)-U(3)$ & $132.8(4)$ & $\mathrm{O}(16)-\mathrm{O}(19)-\mathrm{U}(3)$ & $68.7(4)$ \\
\hline $\mathrm{O}(16)-\mathrm{O}(19)-\mathrm{U}(5)$ & $68.4(4)$ & $U(3)-O(19)-U(5)$ & $123.2(4)$ \\
\hline$O(16)-O(19)-K(2) \# 1$ & $110.1(6)$ & $U(3)-O(19)-K(2) \# 1$ & $136.5(4)$ \\
\hline$U(5)-O(19)-K(2) \# 1$ & $92.7(3)$ & $P(4)-O(20)-U(3) \# 1$ & $131.7(6)$ \\
\hline$P(11)-O(21)-U(3)$ & $142.0(6)$ & $\mathrm{P}(11)-\mathrm{O}(21)-\mathrm{Na}(19)$ & $103.2(7)$ \\
\hline $\mathrm{U}(3)-\mathrm{O}(21)-\mathrm{Na}(19)$ & $98.3(6)$ & $\mathrm{U}(3)-\mathrm{O}(22)-\mathrm{Na}(3)$ & $138.3(5)$ \\
\hline $\mathrm{P}(7)-\mathrm{O}(23)-\mathrm{U}(4)$ & $140.1(5)$ & $\mathrm{P}(7)-\mathrm{O}(23)-\mathrm{K}(1)$ & $94.3(6)$ \\
\hline $\mathrm{U}(4)-\mathrm{O}(23)-\mathrm{K}(1)$ & $85.3(4)$ & $\mathrm{O}(85)-\mathrm{O}(24)-\mathrm{U}(4)$ & $74.9(4)$ \\
\hline $\mathrm{O}(85)-\mathrm{O}(24)-\mathrm{U}(7)$ & $75.0(4)$ & $U(4)-O(24)-U(7)$ & $130.7(4)$ \\
\hline $\mathrm{U}(4)-\mathrm{O}(25)-\mathrm{Na}(11)$ & $146.0(5)$ & $U(4)-O(25)-K(1)$ & $109.3(5)$ \\
\hline $\mathrm{Na}(11)-\mathrm{O}(25)-\mathrm{K}(1)$ & $103.5(5)$ & $O(30)-O(26)-U(7)$ & $74.2(4)$ \\
\hline $\mathrm{O}(30)-\mathrm{O}(26)-\mathrm{U}(5)$ & $75.1(5)$ & $U(7)-O(26)-U(5)$ & $128.4(4)$ \\
\hline$P(2)-O(27)-U(5) \# 1$ & $132.7(5)$ & $P(2)-O(27)-K(2)$ & $84.0(5)$ \\
\hline$U(5) \# 1-O(27)-K(2)$ & $82.6(4)$ & $\mathrm{P}(8)-\mathrm{O}(28)-\mathrm{U}(5) \# 1$ & $133.8(5)$ \\
\hline$U(5)-O(29)-K(2) \# 1$ & $111.5(4)$ & $O(26)-O(30)-U(7)$ & $69.2(4)$ \\
\hline$O(26)-O(30)-U(5)$ & $68.7(4)$ & $U(7)-O(30)-U(5)$ & $121.2(3)$ \\
\hline $\mathrm{O}(26)-\mathrm{O}(30)-\mathrm{K}(3)$ & $112.3(6)$ & $U(7)-O(30)-K(3)$ & $92.1(4)$ \\
\hline$U(5)-O(30)-K(3)$ & $141.6(4)$ & $P(10)-O(31)-U(6)$ & $131.5(6)$ \\
\hline$P(5)-O(32)-U(6)$ & $132.7(6)$ & $P(5)-O(32)-K(3)$ & $126.2(6)$ \\
\hline$U(6)-O(32)-K(3)$ & $95.1(4)$ & $O(49)-O(33)-U(6)$ & $75.3(5)$ \\
\hline $\mathrm{O}(49)-\mathrm{O}(33)-\mathrm{U}(12)$ & $74.7(4)$ & $U(6)-O(33)-U(12)$ & $127.1(4)$ \\
\hline$P(9)-O(34)-U(7)$ & $132.5(5)$ & $\mathrm{O}(39)-\mathrm{O}(35)-\mathrm{U}(8)$ & $69.2(4)$ \\
\hline $\mathrm{O}(39)-\mathrm{O}(35)-\mathrm{U}(10)$ & $70.0(4)$ & $U(8)-O(35)-U(10)$ & $120.5(4)$ \\
\hline $\mathrm{O}(39)-\mathrm{O}(35)-\mathrm{K}(2)$ & $133.8(6)$ & $U(8)-O(35)-K(2)$ & $93.5(4)$ \\
\hline$U(10)-O(35)-K(2)$ & $85.1(3)$ & $\mathrm{U}(7)-\mathrm{O}(36)-\mathrm{Na}(7)$ & $143.5(5)$ \\
\hline$U(7)-O(36)-K(3)$ & $109.8(5)$ & $\mathrm{Na}(7)-\mathrm{O}(36)-\mathrm{K}(3)$ & $106.0(5)$ \\
\hline$P(12)-O(37)-U(7)$ & $134.7(6)$ & $P(12)-O(37)-K(3)$ & $85.2(5)$ \\
\hline$U(7)-O(37)-K(3)$ & $84.4(4)$ & $\mathrm{U}(7)-\mathrm{O}(38)-\mathrm{Na}(3)$ & $140.2(5)$ \\
\hline $\mathrm{O}(35)-\mathrm{O}(39)-\mathrm{U}(8)$ & $74.7(5)$ & $O(35)-O(39)-U(10)$ & $74.0(4)$ \\
\hline$U(8)-O(39)-U(10)$ & $126.2(4)$ & $U(8)-O(41)-K(1)$ & $103.3(5)$ \\
\hline $\mathrm{O}(10)-\mathrm{O}(42)-\mathrm{U}(9) \# 1$ & $70.2(4)$ & $\mathrm{O}(10)-\mathrm{O}(42)-\mathrm{U}(2)$ & $69.9(4)$ \\
\hline$U(9) \# 1-O(42)-U(2)$ & $123.4(3)$ & $\mathrm{U}(3)-\mathrm{O}(43)-\mathrm{Na}(1) \# 1$ & $148.0(5)$ \\
\hline$P(1)-O(44)-U(4)$ & $130.2(5)$ & $\mathrm{U}(4)-\mathrm{O}(45)-\mathrm{Na}(3)$ & $138.5(5)$ \\
\hline $\mathrm{U}(5)-\mathrm{O}(46)-\mathrm{Na}(3)$ & $141.1(6)$ & $U(6)-O(47)-K(3)$ & $104.1(5)$ \\
\hline $\mathrm{O}(33)-\mathrm{O}(49)-\mathrm{U}(6)$ & $68.9(4)$ & $O(33)-O(49)-U(12)$ & $69.6(4)$ \\
\hline$U(6)-O(49)-U(12)$ & $120.2(4)$ & $\mathrm{O}(33)-\mathrm{O}(49)-\mathrm{K}(3)$ & $130.4(6)$ \\
\hline$U(6)-O(49)-K(3)$ & $90.2(4)$ & $U(12)-O(49)-K(3)$ & $85.6(4)$ \\
\hline$P(3)-O(50)-U(8)$ & $130.2(7)$ & $P(3)-O(50)-K(1)$ & $128.4(7)$ \\
\hline $\mathrm{U}(8)-\mathrm{O}(50)-\mathrm{K}(1)$ & $101.0(4)$ & $P(5)-O(51)-U(9)$ & $138.4(6)$ \\
\hline $\mathrm{O}(55)-\mathrm{O}(52)-\mathrm{U}(9) \# 1$ & $74.6(5)$ & $O(55)-O(52)-U(10)$ & $74.3(4)$ \\
\hline$U(9) \# 1-O(52)-U(10)$ & $127.0(4)$ & $P(10)-O(53)-U(9)$ & $137.2(6)$ \\
\hline $\mathrm{U}(9)-\mathrm{O}(54)-\mathrm{Na}(11) \# 1$ & $139.0(5)$ & $\mathrm{O}(52)-\mathrm{O}(55)-\mathrm{U}(9) \# 1$ & $69.0(4)$ \\
\hline
\end{tabular}




\begin{tabular}{|c|c|c|c|}
\hline$O(52)-O(55)-U(10)$ & $69.6(4)$ & $U(9) \# 1-O(55)-U(10)$ & $120.7(4)$ \\
\hline$P(2)-O(57)-U(10)$ & $139.4(5)$ & $P(2)-O(57)-K(2)$ & $111.4(6)$ \\
\hline$U(10)-O(57)-K(2)$ & $100.9(4)$ & $\mathrm{P}(8)-\mathrm{O}(58)-\mathrm{U}(10)$ & $136.4(5)$ \\
\hline$U(10)-O(59)-K(2)$ & $101.3(4)$ & $\mathrm{U}(10)-\mathrm{O}(60)-\mathrm{Na}(13)$ & $146.4(6)$ \\
\hline $\mathrm{U}(11)-\mathrm{O}(61)-\mathrm{Na}(7)$ & $137.1(5)$ & $\mathrm{U}(11)-\mathrm{O}(61)-\mathrm{Na}(1)$ & $139.0(5)$ \\
\hline $\mathrm{Na}(7)-\mathrm{O}(61)-\mathrm{Na}(1)$ & $79.8(5)$ & $P(3)-O(62)-U(11)$ & $140.0(6)$ \\
\hline $\mathrm{O}(64)-\mathrm{O}(63)-\mathrm{U}(11)$ & $73.4(5)$ & $\mathrm{O}(64)-\mathrm{O}(63)-\mathrm{U}(12)$ & $73.3(5)$ \\
\hline$U(11)-O(63)-U(12)$ & $124.9(4)$ & $\mathrm{O}(63)-\mathrm{O}(64)-\mathrm{U}(11)$ & $70.2(5)$ \\
\hline $\mathrm{O}(63)-\mathrm{O}(64)-\mathrm{U}(12)$ & $70.4(5)$ & $U(11)-O(64)-U(12)$ & $121.3(4)$ \\
\hline$P(6)-O(65)-U(11)$ & $140.6(6)$ & $\mathrm{U}(12)-\mathrm{O}(66)-\mathrm{Na}(7)$ & $140.0(5)$ \\
\hline$U(12)-O(66)-K(3)$ & $104.1(5)$ & $\mathrm{Na}(7)-\mathrm{O}(66)-\mathrm{K}(3)$ & $103.3(5)$ \\
\hline$P(9)-O(67)-U(12)$ & $138.6(6)$ & $\mathrm{P}(12)-\mathrm{O}(68)-\mathrm{U}(12)$ & $140.3(6)$ \\
\hline$P(12)-O(68)-K(3)$ & $110.3(6)$ & $\mathrm{U}(12)-\mathrm{O}(68)-\mathrm{K}(3)$ & $97.8(5)$ \\
\hline$P(7)-O(71)-P(1)$ & $123.0(6)$ & $\mathrm{P}(2)-\mathrm{O}(72)-\mathrm{Na}(15)$ & $127.5(6)$ \\
\hline $\mathrm{P}(2)-\mathrm{O}(72)-\mathrm{Na}(10)$ & $124.0(6)$ & $\mathrm{Na}(15)-\mathrm{O}(72)-\mathrm{Na}(10)$ & $93.4(6)$ \\
\hline $\mathrm{P}(2)-\mathrm{O}(72)-\mathrm{Na}(10) \# 3$ & $118.8(6)$ & $\mathrm{Na}(15)-\mathrm{O}(72)-\mathrm{Na}(10) \# 3$ & $89.4(5)$ \\
\hline $\mathrm{Na}(10)-\mathrm{O}(72)-\mathrm{Na}(10) \# 3$ & $94.5(5)$ & $P(8)-O(73)-P(2)$ & $123.9(6)$ \\
\hline$P(4) \# 1-O(74)-P(11)$ & $123.1(6)$ & $\mathrm{P}(7)-\mathrm{O}(76)-\mathrm{Na}(6)$ & $127.8(6)$ \\
\hline $\mathrm{P}(7)-\mathrm{O}(76)-\mathrm{Na}(5)$ & $124.3(6)$ & $\mathrm{Na}(6)-\mathrm{O}(76)-\mathrm{Na}(5)$ & $91.8(5)$ \\
\hline $\mathrm{P}(7)-\mathrm{O}(76)-\mathrm{Na}(6) \# 2$ & $120.2(6)$ & $\mathrm{Na}(6)-\mathrm{O}(76)-\mathrm{Na}(6) \# 2$ & $95.2(5)$ \\
\hline $\mathrm{Na}(5)-\mathrm{O}(76)-\mathrm{Na}(6) \# 2$ & $87.2(5)$ & $\mathrm{P}(11)-\mathrm{O}(77)-\mathrm{Na}(4)$ & $127.3(6)$ \\
\hline $\mathrm{P}(11)-\mathrm{O}(77)-\mathrm{Na}(14)$ & $120.9(6)$ & $\mathrm{Na}(4)-\mathrm{O}(77)-\mathrm{Na}(14)$ & $96.9(6)$ \\
\hline $\mathrm{P}(11)-\mathrm{O}(77)-\mathrm{Na}(4) \# 5$ & $122.0(6)$ & $\mathrm{Na}(4)-\mathrm{O}(77)-\mathrm{Na}(4) \# 5$ & $92.7(5)$ \\
\hline $\mathrm{Na}(14)-\mathrm{O}(77)-\mathrm{Na}(4) \# 5$ & $87.1(6)$ & $P(9)-O(79)-P(12)$ & $124.0(6)$ \\
\hline $\mathrm{P}(12)-\mathrm{O}(80)-\mathrm{Na}(8) \# 6$ & $125.9(6)$ & $\mathrm{P}(12)-\mathrm{O}(80)-\mathrm{Na}(17)$ & $120.7(7)$ \\
\hline $\mathrm{Na}(8) \# 6-\mathrm{O}(80)-\mathrm{Na}(17)$ & $89.8(6)$ & $\mathrm{P}(12)-\mathrm{O}(80)-\mathrm{Na}(8)$ & $120.9(6)$ \\
\hline $\mathrm{Na}(8) \# 6-\mathrm{O}(80)-\mathrm{Na}(8)$ & $98.1(5)$ & $\mathrm{Na}(17)-\mathrm{O}(80)-\mathrm{Na}(8)$ & $93.1(7)$ \\
\hline$P(10)-O(82)-P(5)$ & $121.7(6)$ & $\mathrm{P}(10)-\mathrm{O}(82)-\mathrm{Na}(12)$ & $148.7(5)$ \\
\hline $\mathrm{P}(5)-\mathrm{O}(82)-\mathrm{Na}(12)$ & $89.6(5)$ & $P(6)-O(84)-P(3)$ & $122.3(7)$ \\
\hline $\mathrm{O}(24)-\mathrm{O}(85)-\mathrm{U}(4)$ & $68.5(4)$ & $\mathrm{O}(24)-\mathrm{O}(85)-\mathrm{U}(7)$ & $68.7(4)$ \\
\hline$U(4)-O(85)-U(7)$ & $122.4(4)$ & $\mathrm{O}(24)-\mathrm{O}(85)-\mathrm{K}(1)$ & $109.6(6)$ \\
\hline$U(4)-O(85)-K(1)$ & $92.6(4)$ & $\mathrm{U}(7)-\mathrm{O}(85)-\mathrm{K}(1)$ & $137.6(4)$ \\
\hline$P(6)-O(86)-U(8)$ & 131.1(6) & $P(6)-O(86)-K(2)$ & $124.7(6)$ \\
\hline$U(8)-O(86)-K(2)$ & $104.1(4)$ & $\mathrm{U}(11)-\mathrm{O}(87)-\mathrm{Na}(16)$ & $144.8(4)$ \\
\hline $\mathrm{P}(5)-\mathrm{O}(89)-\mathrm{Na}(12)$ & $111.7(6)$ & $\mathrm{I}(1)-\mathrm{O}(2 \mathrm{I})-\mathrm{Na}(5)$ & $131.2(7)$ \\
\hline $\mathrm{I}(1)-\mathrm{O}(2 \mathrm{I})-\mathrm{Na}(20)$ & $131.0(9)$ & $\mathrm{Na}(5)-\mathrm{O}(2 \mathrm{I})-\mathrm{Na}(20)$ & $95.2(8)$ \\
\hline $\mathrm{I}(1)-\mathrm{O}(2 \mathrm{I})-\mathrm{Na}(21)$ & $122.2(16)$ & $\mathrm{Na}(5)-\mathrm{O}(2 \mathrm{I})-\mathrm{Na}(21)$ & $106.0(16)$ \\
\hline $\mathrm{Na}(20)-\mathrm{O}(2 \mathrm{I})-\mathrm{Na}(21)$ & $27.7(16)$ & $\mathrm{I}(1)-\mathrm{O}(3 \mathrm{I})-\mathrm{Na}(3)$ & $110.1(6)$ \\
\hline $\mathrm{H}(\mathrm{OB})-\mathrm{O}(\mathrm{OW})-\mathrm{H}(\mathrm{OA})$ & $106.2(19)$ & $\mathrm{H}(\mathrm{OB})-\mathrm{O}(\mathrm{OW})-\mathrm{Na}(12)$ & $127.6(16)$ \\
\hline $\mathrm{H}(0 \mathrm{~A})-\mathrm{O}(\mathrm{OW})-\mathrm{Na}(12)$ & $126.2(13)$ & $H(1 B)-O(1 W)-H(1 A)$ & $106.0(18)$ \\
\hline $\mathrm{H}(1 \mathrm{~B})-\mathrm{O}(1 \mathrm{~W})-\mathrm{Na}(12)$ & $112.3(14)$ & $\mathrm{H}(1 \mathrm{~A})-\mathrm{O}(1 \mathrm{~W})-\mathrm{Na}(12)$ & $112.8(14)$ \\
\hline $\mathrm{H}(1 \mathrm{~B})-\mathrm{O}(1 \mathrm{~W})-\mathrm{Na}(2) \# 8$ & $107.7(13)$ & $\mathrm{H}(1 \mathrm{~A})-\mathrm{O}(1 \mathrm{~W})-\mathrm{Na}(2) \# 8$ & $108.6(13)$ \\
\hline $\mathrm{Na}(12)-\mathrm{O}(1 \mathrm{~W})-\mathrm{Na}(2) \# 8$ & $109.0(6)$ & $\mathrm{H}(2 \mathrm{~A})-\mathrm{O}(2 \mathrm{~W})-\mathrm{H}(2 \mathrm{~B})$ & $107(2)$ \\
\hline $\mathrm{H}(2 \mathrm{~A})-\mathrm{O}(2 \mathrm{~W})-\mathrm{Na}(12)$ & $118.0(14)$ & $\mathrm{H}(2 \mathrm{~B})-\mathrm{O}(2 \mathrm{~W})-\mathrm{Na}(12)$ & $113(2)$ \\
\hline
\end{tabular}




\begin{tabular}{|c|c|c|c|}
\hline$H(3 B)-O(3 W)-H(3 A)$ & $106(4)$ & $\mathrm{H}(3 \mathrm{~B})-\mathrm{O}(3 \mathrm{~W})-\mathrm{Na}(12)$ & $120(3)$ \\
\hline $\mathrm{H}(3 \mathrm{~A})-\mathrm{O}(3 \mathrm{~W})-\mathrm{Na}(12)$ & 116.3(19) & $\mathrm{H}(4 \mathrm{~A})-\mathrm{O}(4 \mathrm{~W})-\mathrm{H}(4 \mathrm{~B})$ & $102.3(16)$ \\
\hline $\mathrm{H}(4 \mathrm{~A})-\mathrm{O}(4 \mathrm{~W})-\mathrm{Na}(7)$ & $105.4(11)$ & $\mathrm{H}(4 \mathrm{~B})-\mathrm{O}(4 \mathrm{~W})-\mathrm{Na}(7)$ & $100.1(12)$ \\
\hline $\mathrm{H}(4 \mathrm{~A})-\mathrm{O}(4 \mathrm{~W})-\mathrm{K}(1)$ & 107.7(11) & $\mathrm{H}(4 \mathrm{~B})-\mathrm{O}(4 \mathrm{~W})-\mathrm{K}(1)$ & $140.5(12)$ \\
\hline $\mathrm{Na}(7)-\mathrm{O}(4 \mathrm{~W})-\mathrm{K}(1)$ & $96.1(5)$ & $H(5 A)-O(5 W)-H(5 B)$ & $105.1(16)$ \\
\hline $\mathrm{H}(5 \mathrm{~A})-\mathrm{O}(5 \mathrm{~W})-\mathrm{Na}(1)$ & $99.8(13)$ & $\mathrm{H}(5 \mathrm{~B})-\mathrm{O}(5 \mathrm{~W})-\mathrm{Na}(1)$ & $105.1(13)$ \\
\hline $\mathrm{H}(5 \mathrm{~A})-\mathrm{O}(5 \mathrm{~W})-\mathrm{K}(2)$ & $139.9(12)$ & $H(5 B)-O(5 W)-K(2)$ & $105.1(12)$ \\
\hline $\mathrm{Na}(1)-\mathrm{O}(5 \mathrm{~W})-\mathrm{K}(2)$ & $97.2(5)$ & $\mathrm{O}(5 \mathrm{~W})-\mathrm{H}(5 \mathrm{~B})-\mathrm{K}(2)$ & $57.3(10)$ \\
\hline$H(6 B)-O(6 W)-H(6 A)$ & $104.9(18)$ & $\mathrm{H}(6 \mathrm{~B})-\mathrm{O}(6 \mathrm{~W})-\mathrm{Na}(19)$ & $115.7(15)$ \\
\hline $\mathrm{H}(6 \mathrm{~A})-\mathrm{O}(6 \mathrm{~W})-\mathrm{Na}(19)$ & $115.5(13)$ & $\mathrm{H}(6 \mathrm{~B})-\mathrm{O}(6 \mathrm{~W})-\mathrm{Na}(11)$ & $96.5(13)$ \\
\hline $\mathrm{H}(6 \mathrm{~A})-\mathrm{O}(6 \mathrm{~W})-\mathrm{Na}(11)$ & $103.9(12)$ & $\mathrm{Na}(19)-\mathrm{O}(6 \mathrm{~W})-\mathrm{Na}(11)$ & $117.9(8)$ \\
\hline $\mathrm{O}(6 \mathrm{~W})-\mathrm{H}(6 \mathrm{~B})-\mathrm{Na}(11)$ & $62.5(12)$ & $\mathrm{H}(7 \mathrm{~B})-\mathrm{O}(7 \mathrm{~W})-\mathrm{H}(7 \mathrm{~A})$ & $109(2)$ \\
\hline $\mathrm{H}(7 \mathrm{~B})-\mathrm{O}(7 \mathrm{~W})-\mathrm{K}(3)$ & $132.5(18)$ & $H(7 A)-O(7 W)-K(3)$ & $103.4(14)$ \\
\hline$O(7 W)-H(7 A)-K(3)$ & $58.8(12)$ & $\mathrm{H}(8 \mathrm{~B})-\mathrm{O}(8 \mathrm{~W})-\mathrm{H}(8 \mathrm{~A})$ & $104.9(17)$ \\
\hline $\mathrm{H}(8 \mathrm{~B})-\mathrm{O}(8 \mathrm{~W})-\mathrm{Na}(10)$ & $130.4(13)$ & $\mathrm{H}(8 \mathrm{~A})-\mathrm{O}(8 \mathrm{~W})-\mathrm{Na}(10)$ & $104.6(12)$ \\
\hline $\mathrm{H}(8 \mathrm{~B})-\mathrm{O}(8 \mathrm{~W})-\mathrm{Na}(15)$ & $111.8(13)$ & $\mathrm{H}(8 \mathrm{~A})-\mathrm{O}(8 \mathrm{~W})-\mathrm{Na}(15)$ & $110.2(13)$ \\
\hline $\mathrm{Na}(10)-\mathrm{O}(8 \mathrm{~W})-\mathrm{Na}(15)$ & $93.8(6)$ & $H(9 B)-O(9 W)-H(9 A)$ & $107.6(17)$ \\
\hline $\mathrm{H}(9 \mathrm{~B})-\mathrm{O}(9 \mathrm{~W})-\mathrm{Na}(5) \# 2$ & $121.4(13)$ & $\mathrm{H}(9 \mathrm{~A})-\mathrm{O}(9 \mathrm{~W})-\mathrm{Na}(5) \# 2$ & $116.6(12)$ \\
\hline $\mathrm{H}(9 \mathrm{~B})-\mathrm{O}(9 \mathrm{~W})-\mathrm{Na}(6)$ & $112.7(14)$ & $\mathrm{H}(9 \mathrm{~A})-\mathrm{O}(9 \mathrm{~W})-\mathrm{Na}(6)$ & $104.9(13)$ \\
\hline $\mathrm{Na}(5) \# 2-\mathrm{O}(9 \mathrm{~W})-\mathrm{Na}(6)$ & $91.5(5)$ & $H(10 B)-O(10 W)-H(10 A)$ & $103.0(16)$ \\
\hline $\mathrm{H}(10 \mathrm{~B})-\mathrm{O}(10 \mathrm{~W})-\mathrm{Na}(2)$ & $125.4(13)$ & $\mathrm{H}(10 \mathrm{~A})-\mathrm{O}(10 \mathrm{~W})-\mathrm{Na}(2)$ & $118.0(11)$ \\
\hline$H(11 A)-O(11 W)-H(11 B)$ & $105.6(17)$ & $\mathrm{H}(11 \mathrm{~A})-\mathrm{O}(11 \mathrm{~W})-\mathrm{Na}(9) \# 7$ & $110.7(12)$ \\
\hline $\mathrm{H}(11 \mathrm{~B})-\mathrm{O}(11 \mathrm{~W})-\mathrm{Na}(9) \# 7$ & $107.9(12)$ & $\mathrm{H}(11 \mathrm{~A})-\mathrm{O}(11 \mathrm{~W})-\mathrm{Na}(13)$ & $128.8(13)$ \\
\hline $\mathrm{H}(11 \mathrm{~B})-\mathrm{O}(11 \mathrm{~W})-\mathrm{Na}(13)$ & $106.6(11)$ & $\mathrm{Na}(9) \# 7-\mathrm{O}(11 \mathrm{~W})-\mathrm{Na}(13)$ & $95.9(5)$ \\
\hline$H(12 A)-O(12 W)-H(12 B)$ & $108.9(18)$ & $\mathrm{H}(12 \mathrm{~A})-\mathrm{O}(12 \mathrm{~W})-\mathrm{Na}(7)$ & $102.4(12)$ \\
\hline $\mathrm{H}(12 \mathrm{~B})-\mathrm{O}(12 \mathrm{~W})-\mathrm{Na}(7)$ & $101.9(12)$ & $\mathrm{H}(12 \mathrm{~A})-\mathrm{O}(12 \mathrm{~W})-\mathrm{Na}(1)$ & $144.8(13)$ \\
\hline $\mathrm{H}(12 \mathrm{~B})-\mathrm{O}(12 \mathrm{~W})-\mathrm{Na}(1)$ & $101.8(13)$ & $\mathrm{Na}(7)-\mathrm{O}(12 \mathrm{~W})-\mathrm{Na}(1)$ & $87.2(6)$ \\
\hline$H(13 A)-O(13 W)-H(13 B)$ & $107.5(16)$ & $\mathrm{H}(13 \mathrm{~A})-\mathrm{O}(13 \mathrm{~W})-\mathrm{Na}(9) \# 7$ & $104.6(12)$ \\
\hline $\mathrm{H}(13 \mathrm{~B})-\mathrm{O}(13 \mathrm{~W})-\mathrm{Na}(9) \# 7$ & $119.9(11)$ & $\mathrm{H}(13 \mathrm{~A})-\mathrm{O}(13 \mathrm{~W})-\mathrm{Na}(13)$ & $117.2(12)$ \\
\hline $\mathrm{H}(13 \mathrm{~B})-\mathrm{O}(13 \mathrm{~W})-\mathrm{Na}(13)$ & $110.1(12)$ & $\mathrm{Na}(9) \# 7-\mathrm{O}(13 \mathrm{~W})-\mathrm{Na}(13)$ & $97.8(6)$ \\
\hline$H(14 B)-O(14 W)-H(14 A)$ & $106.9(18)$ & $\mathrm{H}(14 \mathrm{~B})-\mathrm{O}(14 \mathrm{~W})-\mathrm{Na}(9)$ & $114.7(13)$ \\
\hline $\mathrm{H}(14 \mathrm{~A})-\mathrm{O}(14 \mathrm{~W})-\mathrm{Na}(9)$ & $119.4(13)$ & $\mathrm{H}(14 \mathrm{~B})-\mathrm{O}(14 \mathrm{~W})-\mathrm{Na}(2)$ & $110.3(13)$ \\
\hline $\mathrm{H}(14 \mathrm{~A})-\mathrm{O}(14 \mathrm{~W})-\mathrm{Na}(2)$ & $109.4(12)$ & $\mathrm{Na}(9)-\mathrm{O}(14 \mathrm{~W})-\mathrm{Na}(2)$ & $95.4(5)$ \\
\hline$H(15 B)-O(15 W)-H(15 A)$ & $108.2(19)$ & $\mathrm{H}(15 \mathrm{~B})-\mathrm{O}(15 \mathrm{~W})-\mathrm{Na}(6)$ & $136.8(15)$ \\
\hline $\mathrm{H}(15 \mathrm{~A})-\mathrm{O}(15 \mathrm{~W})-\mathrm{Na}(6)$ & $112.2(14)$ & $\mathrm{H}(15 \mathrm{~B})-\mathrm{O}(15 \mathrm{~W})-\mathrm{Na}(5)$ & $99.9(15)$ \\
\hline $\mathrm{H}(15 \mathrm{~A})-\mathrm{O}(15 \mathrm{~W})-\mathrm{Na}(5)$ & $96.5(14)$ & $\mathrm{Na}(6)-\mathrm{O}(15 \mathrm{~W})-\mathrm{Na}(5)$ & $90.0(5)$ \\
\hline $\mathrm{H}(15 \mathrm{~B})-\mathrm{O}(15 \mathrm{~W})-\mathrm{K}(1) \# 2$ & $80.5(14)$ & $H(15 A)-O(15 W)-K(1) \# 2$ & $58.9(13)$ \\
\hline $\mathrm{Na}(6)-\mathrm{O}(15 \mathrm{~W})-\mathrm{K}(1) \# 2$ & $108.1(5)$ & $\mathrm{Na}(5)-\mathrm{O}(15 \mathrm{~W})-\mathrm{K}(1) \# 2$ & $153.3(5)$ \\
\hline $\mathrm{O}(15 \mathrm{~W})-\mathrm{H}(15 \mathrm{~A})-\mathrm{K}(1) \# 2$ & $104.8(14)$ & $H(16 B)-O(16 W)-H(16 A)$ & 109.3(19) \\
\hline $\mathrm{H}(16 \mathrm{~B})-\mathrm{O}(16 \mathrm{~W})-\mathrm{Na}(14)$ & $122.5(15)$ & $\mathrm{H}(16 \mathrm{~A})-\mathrm{O}(16 \mathrm{~W})-\mathrm{Na}(14)$ & $120.3(14)$ \\
\hline $\mathrm{H}(16 \mathrm{~B})-\mathrm{O}(16 \mathrm{~W})-\mathrm{Na}(4) \# 5$ & $103.2(14)$ & $\mathrm{H}(16 \mathrm{~A})-\mathrm{O}(16 \mathrm{~W})-\mathrm{Na}(4) \# 5$ & $106.7(14)$ \\
\hline $\mathrm{Na}(14)-\mathrm{O}(16 \mathrm{~W})-\mathrm{Na}(4) \# 5$ & $89.3(6)$ & $H(17 B)-O(17 W)-H(17 A)$ & $107(3)$ \\
\hline $\mathrm{H}(17 \mathrm{~B})-\mathrm{O}(17 \mathrm{~W})-\mathrm{Na}(18)$ & $118(2)$ & $\mathrm{H}(17 \mathrm{~A})-\mathrm{O}(17 \mathrm{~W})-\mathrm{Na}(18)$ & $127.9(18)$ \\
\hline $\mathrm{H}(17 \mathrm{~B})-\mathrm{O}(17 \mathrm{~W})-\mathrm{Na}(15)$ & $109(2)$ & $\mathrm{H}(17 \mathrm{~A})-\mathrm{O}(17 \mathrm{~W})-\mathrm{Na}(15)$ & $104.0(17)$ \\
\hline
\end{tabular}




\begin{tabular}{|c|c|c|c|}
\hline $\mathrm{Na}(18)-\mathrm{O}(17 \mathrm{~W})-\mathrm{Na}(15)$ & $85.9(8)$ & $\mathrm{H}(18 \mathrm{~A})-\mathrm{O}(18 \mathrm{~W})-\mathrm{H}(18 \mathrm{~B})$ & $105.3(18)$ \\
\hline $\mathrm{H}(18 \mathrm{~A})-\mathrm{O}(18 \mathrm{~W})-\mathrm{Na}(4)$ & $110.8(13)$ & $\mathrm{H}(18 \mathrm{~B})-\mathrm{O}(18 \mathrm{~W})-\mathrm{Na}(4)$ & $107.0(14)$ \\
\hline$H(19 B)-O(19 W)-H(19 A)$ & $102(2)$ & $\mathrm{H}(19 \mathrm{~B})-\mathrm{O}(19 \mathrm{~W})-\mathrm{Na}(9)$ & $130.7(18)$ \\
\hline $\mathrm{H}(19 \mathrm{~A})-\mathrm{O}(19 \mathrm{~W})-\mathrm{Na}(9)$ & $105.1(14)$ & $\mathrm{H}(20 \mathrm{~B})-\mathrm{O}(20 \mathrm{~W})-\mathrm{H}(20 \mathrm{C})$ & $38(3)$ \\
\hline$H(20 B)-O(20 W)-H(20 A)$ & $111(3)$ & $H(20 C)-O(20 W)-H(20 A)$ & $108(3)$ \\
\hline $\mathrm{H}(20 \mathrm{~B})-\mathrm{O}(20 \mathrm{~W})-\mathrm{Na}(4)$ & $107(3)$ & $\mathrm{H}(20 \mathrm{C})-\mathrm{O}(20 \mathrm{~W})-\mathrm{Na}(4)$ & $110(2)$ \\
\hline $\mathrm{H}(20 \mathrm{~A})-\mathrm{O}(20 \mathrm{~W})-\mathrm{Na}(4)$ & $139.8(14)$ & $\mathrm{H}(20 \mathrm{~B})-\mathrm{O}(20 \mathrm{~W})-\mathrm{Na}(19) \# 5$ & $57(3)$ \\
\hline $\mathrm{H}(20 \mathrm{C})-\mathrm{O}(20 \mathrm{~W})-\mathrm{Na}(19) \# 5$ & $95(3)$ & $\mathrm{H}(20 \mathrm{~A})-\mathrm{O}(20 \mathrm{~W})-\mathrm{Na}(19) \# 5$ & $94.5(14)$ \\
\hline $\mathrm{Na}(4)-\mathrm{O}(20 \mathrm{~W})-\mathrm{Na}(19) \# 5$ & $95.2(7)$ & $H(20 C)-H(20 B)-O(20 W)$ & $71(5)$ \\
\hline $\mathrm{H}(20 \mathrm{C})-\mathrm{H}(20 \mathrm{~B})-\mathrm{Na}(19) \# 5$ & 169(6) & $\mathrm{O}(20 \mathrm{~W})-\mathrm{H}(20 \mathrm{~B})-\mathrm{Na}(19) \# 5$ & $100(4)$ \\
\hline$H(20 B)-H(20 C)-O(20 W)$ & $70(5)$ & $H(21 A)-O(21 W)-H(21 B)$ & $107(2)$ \\
\hline $\mathrm{H}(21 \mathrm{~A})-\mathrm{O}(21 \mathrm{~W})-\mathrm{Na}(10)$ & $107.0(15)$ & $\mathrm{H}(21 \mathrm{~B})-\mathrm{O}(21 \mathrm{~W})-\mathrm{Na}(10)$ & $122(2)$ \\
\hline $\mathrm{H}(21 \mathrm{~A})-\mathrm{O}(21 \mathrm{~W})-\mathrm{Na}(15) \# 3$ & $108.9(15)$ & $\mathrm{H}(21 \mathrm{~B})-\mathrm{O}(21 \mathrm{~W})-\mathrm{Na}(15) \# 3$ & 119.7(19) \\
\hline $\mathrm{Na}(10)-\mathrm{O}(21 \mathrm{~W})-\mathrm{Na}(15) \# 3$ & $90.9(6)$ & $H(22 B)-O(22 W)-H(22 A)$ & $103(2)$ \\
\hline $\mathrm{H}(22 \mathrm{~B})-\mathrm{O}(22 \mathrm{~W})-\mathrm{Na}(10)$ & $130.2(17)$ & $\mathrm{H}(22 \mathrm{~A})-\mathrm{O}(22 \mathrm{~W})-\mathrm{Na}(10)$ & $106.0(14)$ \\
\hline $\mathrm{H}(23 \mathrm{~B})-\mathrm{O}(23 \mathrm{~W})-\mathrm{H}(23 \mathrm{~A})$ & 109.1(17) & $\mathrm{H}(23 \mathrm{~B})-\mathrm{O}(23 \mathrm{~W})-\mathrm{Na}(13)$ & $107.7(14)$ \\
\hline $\mathrm{H}(23 \mathrm{~A})-\mathrm{O}(23 \mathrm{~W})-\mathrm{Na}(13)$ & $99.2(13)$ & $\mathrm{H}(24 \mathrm{~B})-\mathrm{O}(24 \mathrm{~W})-\mathrm{H}(24 \mathrm{~A})$ & $110.5(19)$ \\
\hline $\mathrm{H}(24 \mathrm{~B})-\mathrm{O}(24 \mathrm{~W})-\mathrm{Na}(17) \# 6$ & $116.3(15)$ & $\mathrm{H}(24 \mathrm{~A})-\mathrm{O}(24 \mathrm{~W})-\mathrm{Na}(17) \# 6$ & $119.1(13)$ \\
\hline $\mathrm{H}(24 \mathrm{~B})-\mathrm{O}(24 \mathrm{~W})-\mathrm{Na}(8)$ & $103.7(14)$ & $\mathrm{H}(24 \mathrm{~A})-\mathrm{O}(24 \mathrm{~W})-\mathrm{Na}(8)$ & $114.0(14)$ \\
\hline $\mathrm{Na}(17) \# 6-\mathrm{O}(24 \mathrm{~W})-\mathrm{Na}(8)$ & $90.6(7)$ & $H(25 B)-O(25 W)-H(25 A)$ & $106(2)$ \\
\hline $\mathrm{H}(25 \mathrm{~B})-\mathrm{O}(25 \mathrm{~W})-\mathrm{Na}(2)$ & $106.1(13)$ & $\mathrm{H}(25 \mathrm{~A})-\mathrm{O}(25 \mathrm{~W})-\mathrm{Na}(2)$ & $113.1(17)$ \\
\hline$H(26 B)-O(26 W)-H(26 A)$ & $100(3)$ & $\mathrm{H}(26 \mathrm{~B})-\mathrm{O}(26 \mathrm{~W})-\mathrm{Na}(9)$ & $110(2)$ \\
\hline $\mathrm{H}(26 \mathrm{~A})-\mathrm{O}(26 \mathrm{~W})-\mathrm{Na}(9)$ & $106.3(16)$ & $\mathrm{H}(27 \mathrm{~B})-\mathrm{O}(27 \mathrm{~W})-\mathrm{H}(27 \mathrm{~A})$ & $109(2)$ \\
\hline $\mathrm{H}(27 \mathrm{~B})-\mathrm{O}(27 \mathrm{~W})-\mathrm{Na}(4)$ & $115.9(15)$ & $\mathrm{H}(27 \mathrm{~A})-\mathrm{O}(27 \mathrm{~W})-\mathrm{Na}(4)$ & $134.8(14)$ \\
\hline $\mathrm{H}(27 \mathrm{~B})-\mathrm{O}(27 \mathrm{~W})-\mathrm{Na}(19) \# 5$ & $83.4(16)$ & $\mathrm{H}(27 \mathrm{~A})-\mathrm{O}(27 \mathrm{~W})-\mathrm{Na}(19) \# 5$ & $104.9(17)$ \\
\hline $\mathrm{Na}(4)-\mathrm{O}(27 \mathrm{~W})-\mathrm{Na}(19) \# 5$ & $87.9(7)$ & $\mathrm{H}(27 \mathrm{~B})-\mathrm{O}(27 \mathrm{~W})-\mathrm{Na}(14)$ & $90.1(16)$ \\
\hline $\mathrm{H}(27 \mathrm{~A})-\mathrm{O}(27 \mathrm{~W})-\mathrm{Na}(14)$ & $87.7(16)$ & $\mathrm{Na}(4)-\mathrm{O}(27 \mathrm{~W})-\mathrm{Na}(14)$ & $85.0(6)$ \\
\hline $\mathrm{Na}(19) \# 5-\mathrm{O}(27 \mathrm{~W})-\mathrm{Na}(14)$ & $167.1(8)$ & $\mathrm{H}(28 \mathrm{~B})-\mathrm{O}(28 \mathrm{~W})-\mathrm{H}(28 \mathrm{~A})$ & $104(2)$ \\
\hline $\mathrm{H}(28 \mathrm{~B})-\mathrm{O}(28 \mathrm{~W})-\mathrm{Na}(8)$ & $128.3(14)$ & $\mathrm{H}(28 \mathrm{~A})-\mathrm{O}(28 \mathrm{~W})-\mathrm{Na}(8)$ & $104.7(14)$ \\
\hline $\mathrm{H}(29 \mathrm{~A})-\mathrm{O}(29 \mathrm{~W})-\mathrm{H}(29 B)$ & $106(2)$ & $\mathrm{H}(29 \mathrm{~A})-\mathrm{O}(29 \mathrm{~W})-\mathrm{Na}(15)$ & $103.9(12)$ \\
\hline $\mathrm{H}(29 \mathrm{~B})-\mathrm{O}(29 \mathrm{~W})-\mathrm{Na}(15)$ & $94.1(14)$ & $\mathrm{H}(29 \mathrm{~A})-\mathrm{O}(29 \mathrm{~W})-\mathrm{Na}(13)$ & $112.9(12)$ \\
\hline $\mathrm{H}(29 \mathrm{~B})-\mathrm{O}(29 \mathrm{~W})-\mathrm{Na}(13)$ & $87.0(15)$ & $\mathrm{Na}(15)-\mathrm{O}(29 \mathrm{~W})-\mathrm{Na}(13)$ & $141.4(6)$ \\
\hline $\mathrm{H}(29 \mathrm{~A})-\mathrm{O}(29 \mathrm{~W})-\mathrm{Na}(18)$ & $111.4(14)$ & $\mathrm{H}(29 \mathrm{~B})-\mathrm{O}(29 \mathrm{~W})-\mathrm{Na}(18)$ & $142.9(17)$ \\
\hline $\mathrm{Na}(15)-\mathrm{O}(29 \mathrm{~W})-\mathrm{Na}(18)$ & $75.5(7)$ & $\mathrm{Na}(13)-\mathrm{O}(29 \mathrm{~W})-\mathrm{Na}(18)$ & $80.6(6)$ \\
\hline $\mathrm{H}(30 \mathrm{~A})-\mathrm{O}(30 \mathrm{~W})-\mathrm{H}(30 \mathrm{~B})$ & $110(2)$ & $\mathrm{H}(30 \mathrm{~A})-\mathrm{O}(30 \mathrm{~W})-\mathrm{Na}(18)$ & $126.5(17)$ \\
\hline $\mathrm{H}(30 \mathrm{~B})-\mathrm{O}(30 \mathrm{~W})-\mathrm{Na}(18)$ & $98.0(17)$ & $\mathrm{H}(30 \mathrm{~A})-\mathrm{O}(30 \mathrm{~W})-\mathrm{Na}(13)$ & $109.4(13)$ \\
\hline $\mathrm{H}(30 \mathrm{~B})-\mathrm{O}(30 \mathrm{~W})-\mathrm{Na}(13)$ & $118.2(17)$ & $\mathrm{Na}(18)-\mathrm{O}(30 \mathrm{~W})-\mathrm{Na}(13)$ & $94.6(8)$ \\
\hline $\mathrm{O}(30 \mathrm{~W})-\mathrm{H}(30 \mathrm{~B})-\mathrm{Na}(18)$ & $60.1(16)$ & $H(31 B)-O(31 W)-H(31 A)$ & $109(2)$ \\
\hline $\mathrm{H}(31 \mathrm{~B})-\mathrm{O}(31 \mathrm{~W})-\mathrm{Na}(10)$ & $112.7(19)$ & $\mathrm{H}(31 \mathrm{~A})-\mathrm{O}(31 \mathrm{~W})-\mathrm{Na}(10)$ & $122.1(17)$ \\
\hline $\mathrm{H}(31 \mathrm{~B})-\mathrm{O}(31 \mathrm{~W})-\mathrm{K}(2) \# 3$ & $85.3(19)$ & $H(31 A)-O(31 W)-K(2) \# 3$ & $104.8(18)$ \\
\hline $\mathrm{Na}(10)-\mathrm{O}(31 \mathrm{~W})-\mathrm{K}(2) \# 3$ & $117.1(6)$ & $\mathrm{O}(31 \mathrm{~W})-\mathrm{H}(31 \mathrm{~B})-\mathrm{K}(2) \# 3$ & $76.1(18)$ \\
\hline $\mathrm{H}(32 \mathrm{~A})-\mathrm{O}(32 \mathrm{~W})-\mathrm{H}(32 \mathrm{C})$ & 107(3) & $\mathrm{H}(32 \mathrm{~A})-\mathrm{O}(32 \mathrm{~W})-\mathrm{H}(32 \mathrm{~B})$ & $120(3)$ \\
\hline $\mathrm{H}(32 \mathrm{C})-\mathrm{O}(32 \mathrm{~W})-\mathrm{H}(32 \mathrm{~B})$ & $63(3)$ & $\mathrm{H}(32 \mathrm{~A})-\mathrm{O}(32 \mathrm{~W})-\mathrm{Na}(11)$ & $105.2(14)$ \\
\hline $\mathrm{H}(32 \mathrm{C})-\mathrm{O}(32 \mathrm{~W})-\mathrm{Na}(11)$ & $94(2)$ & $\mathrm{H}(32 \mathrm{~B})-\mathrm{O}(32 \mathrm{~W})-\mathrm{Na}(11)$ & $134(3)$ \\
\hline
\end{tabular}




\begin{tabular}{|c|c|c|c|}
\hline $\mathrm{O}(32 \mathrm{~W})-\mathrm{H}(32 \mathrm{~B})-\mathrm{H}(32 \mathrm{C})$ & $58.1(17)$ & $\mathrm{O}(32 \mathrm{~W})-\mathrm{H}(32 \mathrm{C})-\mathrm{H}(32 \mathrm{~B})$ & $58.4(17)$ \\
\hline $\mathrm{O}(32 \mathrm{~W})-\mathrm{H}(32 \mathrm{C})-\mathrm{Na}(11)$ & $64(2)$ & $\mathrm{H}(32 \mathrm{~B})-\mathrm{H}(32 \mathrm{C})-\mathrm{Na}(11)$ & $110(3)$ \\
\hline $\mathrm{H}(33 \mathrm{~B})-\mathrm{O}(33 \mathrm{~W})-\mathrm{H}(33 \mathrm{~A})$ & $114(3)$ & $\mathrm{H}(33 \mathrm{~B})-\mathrm{O}(33 \mathrm{~W})-\mathrm{H}(33 \mathrm{C})$ & $34(4)$ \\
\hline $\mathrm{H}(33 \mathrm{~A})-\mathrm{O}(33 \mathrm{~W})-\mathrm{H}(33 \mathrm{C})$ & $117(4)$ & $\mathrm{H}(33 \mathrm{~B})-\mathrm{O}(33 \mathrm{~W})-\mathrm{Na}(1)$ & $133(2)$ \\
\hline $\mathrm{H}(33 \mathrm{~A})-\mathrm{O}(33 \mathrm{~W})-\mathrm{Na}(1)$ & $113.1(15)$ & $\mathrm{H}(33 \mathrm{C})-\mathrm{O}(33 \mathrm{~W})-\mathrm{Na}(1)$ & $122(4)$ \\
\hline $\mathrm{H}(33 \mathrm{~B})-\mathrm{O}(33 \mathrm{~W})-\mathrm{K}(2)$ & $83(3)$ & $H(33 A)-O(33 W)-K(2)$ & $91.3(17)$ \\
\hline $\mathrm{H}(33 \mathrm{C})-\mathrm{O}(33 \mathrm{~W})-\mathrm{K}(2)$ & $117(4)$ & $\mathrm{Na}(1)-\mathrm{O}(33 \mathrm{~W})-\mathrm{K}(2)$ & $88.7(6)$ \\
\hline$H(33 C)-H(33 B)-O(33 W)$ & $73(2)$ & $H(33 C)-H(33 B)-K(2)$ & $150(4)$ \\
\hline $\mathrm{O}(33 \mathrm{~W})-\mathrm{H}(33 \mathrm{~B})-\mathrm{K}(2)$ & $79(3)$ & $\mathrm{H}(33 \mathrm{~B})-\mathrm{H}(33 \mathrm{C})-\mathrm{O}(33 \mathrm{~W})$ & $72(2)$ \\
\hline $\mathrm{H}(34 \mathrm{~B})-\mathrm{O}(34 \mathrm{~W})-\mathrm{H}(34 \mathrm{~A})$ & $110(2)$ & $\mathrm{H}(34 \mathrm{~B})-\mathrm{O}(34 \mathrm{~W})-\mathrm{Na}(5)$ & $122(2)$ \\
\hline $\mathrm{H}(34 \mathrm{~A})-\mathrm{O}(34 \mathrm{~W})-\mathrm{Na}(5)$ & $111.0(15)$ & $\mathrm{H}(35 \mathrm{~B})-\mathrm{O}(35 \mathrm{~W})-\mathrm{H}(35 \mathrm{~A})$ & $108(2)$ \\
\hline $\mathrm{H}(35 \mathrm{~B})-\mathrm{O}(35 \mathrm{~W})-\mathrm{Na}(8)$ & $113(2)$ & $\mathrm{H}(35 \mathrm{~A})-\mathrm{O}(35 \mathrm{~W})-\mathrm{Na}(8)$ & $122.6(16)$ \\
\hline$H(36 B)-O(36 W)-H(36 A)$ & $103(2)$ & $\mathrm{H}(36 \mathrm{~B})-\mathrm{O}(36 \mathrm{~W})-\mathrm{Na}(9)$ & $100.6(13)$ \\
\hline $\mathrm{H}(36 \mathrm{~A})-\mathrm{O}(36 \mathrm{~W})-\mathrm{Na}(9)$ & $109.5(15)$ & $\mathrm{H}(36 \mathrm{~B})-\mathrm{O}(36 \mathrm{~W})-\mathrm{Na}(2)$ & $127.7(15)$ \\
\hline $\mathrm{H}(36 \mathrm{~A})-\mathrm{O}(36 \mathrm{~W})-\mathrm{Na}(2)$ & $116.6(15)$ & $\mathrm{Na}(9)-\mathrm{O}(36 \mathrm{~W})-\mathrm{Na}(2)$ & $96.6(5)$ \\
\hline $\mathrm{H}(37 \mathrm{~A})-\mathrm{O}(37 \mathrm{~W})-\mathrm{H}(37 \mathrm{~B})$ & $95(3)$ & $\mathrm{H}(37 \mathrm{~A})-\mathrm{O}(37 \mathrm{~W})-\mathrm{Na}(18) \# 11$ & $118.2(18)$ \\
\hline $\mathrm{H}(37 \mathrm{~B})-\mathrm{O}(37 \mathrm{~W})-\mathrm{Na}(18) \# 11$ & $104(2)$ & $\mathrm{H}(37 \mathrm{~A})-\mathrm{O}(37 \mathrm{~W})-\mathrm{Na}(14)$ & $100.0(18)$ \\
\hline $\mathrm{H}(37 \mathrm{~B})-\mathrm{O}(37 \mathrm{~W})-\mathrm{Na}(14)$ & $116(2)$ & $\mathrm{Na}(18) \# 11-\mathrm{O}(37 \mathrm{~W})-\mathrm{Na}(14)$ & $120.8(9)$ \\
\hline $\mathrm{H}(38 \mathrm{~B})-\mathrm{O}(38 \mathrm{~W})-\mathrm{H}(38 \mathrm{C})$ & $122(4)$ & $\mathrm{H}(38 \mathrm{~B})-\mathrm{O}(38 \mathrm{~W})-\mathrm{H}(38 \mathrm{~A})$ & $89(4)$ \\
\hline $\mathrm{H}(38 \mathrm{C})-\mathrm{O}(38 \mathrm{~W})-\mathrm{H}(38 \mathrm{~A})$ & $36(3)$ & $\mathrm{H}(38 \mathrm{~B})-\mathrm{O}(38 \mathrm{~W})-\mathrm{Na}(15)$ & $117(2)$ \\
\hline $\mathrm{H}(38 \mathrm{C})-\mathrm{O}(38 \mathrm{~W})-\mathrm{Na}(15)$ & $112(3)$ & $\mathrm{H}(38 \mathrm{~A})-\mathrm{O}(38 \mathrm{~W})-\mathrm{Na}(15)$ & $126(3)$ \\
\hline $\mathrm{H}(38 \mathrm{~B})-\mathrm{O}(38 \mathrm{~W})-\mathrm{Na}(18)$ & $117(3)$ & $\mathrm{H}(38 \mathrm{C})-\mathrm{O}(38 \mathrm{~W})-\mathrm{Na}(18)$ & $100(3)$ \\
\hline $\mathrm{H}(38 \mathrm{~A})-\mathrm{O}(38 \mathrm{~W})-\mathrm{Na}(18)$ & $132(3)$ & $\mathrm{Na}(15)-\mathrm{O}(38 \mathrm{~W})-\mathrm{Na}(18)$ & $80.1(7)$ \\
\hline $\mathrm{H}(38 \mathrm{C})-\mathrm{H}(38 \mathrm{~A})-\mathrm{O}(38 \mathrm{~W})$ & $72(2)$ & $\mathrm{H}(38 \mathrm{~A})-\mathrm{H}(38 \mathrm{C})-\mathrm{O}(38 \mathrm{~W})$ & $72(2)$ \\
\hline$H(39 A)-O(39 W)-H(39 B)$ & $102(3)$ & $\mathrm{H}(39 \mathrm{~A})-\mathrm{O}(39 \mathrm{~W})-\mathrm{Na}(1)$ & $141(2)$ \\
\hline $\mathrm{H}(39 \mathrm{~B})-\mathrm{O}(39 \mathrm{~W})-\mathrm{Na}(1)$ & $103(2)$ & $\mathrm{H}(39 \mathrm{~A})-\mathrm{O}(39 \mathrm{~W})-\mathrm{Na}(7)$ & $125(2)$ \\
\hline $\mathrm{H}(39 \mathrm{~B})-\mathrm{O}(39 \mathrm{~W})-\mathrm{Na}(7)$ & $97(2)$ & $\mathrm{Na}(1)-\mathrm{O}(39 \mathrm{~W})-\mathrm{Na}(7)$ & $80.5(5)$ \\
\hline $\mathrm{H}(39 \mathrm{~A})-\mathrm{O}(39 \mathrm{~W})-\mathrm{K}(2)$ & $71(2)$ & $\mathrm{H}(39 \mathrm{~B})-\mathrm{O}(39 \mathrm{~W})-\mathrm{K}(2)$ & $93(2)$ \\
\hline $\mathrm{Na}(1)-\mathrm{O}(39 \mathrm{~W})-\mathrm{K}(2)$ & $78.5(6)$ & $\mathrm{Na}(7)-\mathrm{O}(39 \mathrm{~W})-\mathrm{K}(2)$ & $158.1(6)$ \\
\hline $\mathrm{H}(40 \mathrm{~B})-\mathrm{O}(40 \mathrm{~W})-\mathrm{H}(40 \mathrm{~A})$ & $109(4)$ & $\mathrm{H}(40 \mathrm{~B})-\mathrm{O}(40 \mathrm{~W})-\mathrm{Na}(16)$ & $122(4)$ \\
\hline $\mathrm{H}(40 \mathrm{~A})-\mathrm{O}(40 \mathrm{~W})-\mathrm{Na}(16)$ & $109.5(17)$ & $\mathrm{H}(41 \mathrm{C})-\mathrm{O}(41 \mathrm{~W})-\mathrm{H}(41 \mathrm{~B})$ & $63(6)$ \\
\hline $\mathrm{H}(41 \mathrm{C})-\mathrm{O}(41 \mathrm{~W})-\mathrm{H}(41 \mathrm{~A})$ & $94(5)$ & $\mathrm{H}(41 \mathrm{~B})-\mathrm{O}(41 \mathrm{~W})-\mathrm{H}(41 \mathrm{~A})$ & $96(5)$ \\
\hline $\mathrm{H}(41 \mathrm{C})-\mathrm{O}(41 \mathrm{~W})-\mathrm{Na}(8)$ & $114(5)$ & $\mathrm{H}(41 \mathrm{~B})-\mathrm{O}(41 \mathrm{~W})-\mathrm{Na}(8)$ & $161(5)$ \\
\hline $\mathrm{H}(41 \mathrm{~A})-\mathrm{O}(41 \mathrm{~W})-\mathrm{Na}(8)$ & $103(2)$ & $\mathrm{H}(41 \mathrm{C})-\mathrm{O}(41 \mathrm{~W})-\mathrm{Na}(17)$ & $119(5)$ \\
\hline $\mathrm{H}(41 \mathrm{~B})-\mathrm{O}(41 \mathrm{~W})-\mathrm{Na}(17)$ & $76(5)$ & $\mathrm{H}(41 \mathrm{~A})-\mathrm{O}(41 \mathrm{~W})-\mathrm{Na}(17)$ & $135(2)$ \\
\hline $\mathrm{Na}(8)-\mathrm{O}(41 \mathrm{~W})-\mathrm{Na}(17)$ & $90.6(8)$ & $\mathrm{O}(41 \mathrm{~W})-\mathrm{H}(41 \mathrm{~B})-\mathrm{H}(41 \mathrm{C})$ & $58(3)$ \\
\hline $\mathrm{O}(41 \mathrm{~W})-\mathrm{H}(41 \mathrm{~B})-\mathrm{Na}(17)$ & $81(5)$ & $\mathrm{H}(41 \mathrm{C})-\mathrm{H}(41 \mathrm{~B})-\mathrm{Na}(17)$ & $120(6)$ \\
\hline $\mathrm{O}(41 \mathrm{~W})-\mathrm{H}(41 \mathrm{C})-\mathrm{H}(41 \mathrm{~B})$ & $59(3)$ & $\mathrm{H}(42 \mathrm{~A})-\mathrm{O}(42 \mathrm{~W})-\mathrm{H}(42 \mathrm{~B})$ & $102(4)$ \\
\hline $\mathrm{H}(42 \mathrm{~A})-\mathrm{O}(42 \mathrm{~W})-\mathrm{Na}(14)$ & $128(2)$ & $\mathrm{H}(42 \mathrm{~B})-\mathrm{O}(42 \mathrm{~W})-\mathrm{Na}(14)$ & $127(3)$ \\
\hline $\mathrm{H}(43 \mathrm{~B})-\mathrm{O}(43 \mathrm{~W})-\mathrm{H}(43 \mathrm{~A})$ & $94(4)$ & $\mathrm{H}(43 \mathrm{~B})-\mathrm{O}(43 \mathrm{~W})-\mathrm{Na}(5)$ & $131(3)$ \\
\hline $\mathrm{H}(43 \mathrm{~A})-\mathrm{O}(43 \mathrm{~W})-\mathrm{Na}(5)$ & $98(2)$ & $\mathrm{H}(43 \mathrm{~B})-\mathrm{O}(43 \mathrm{~W})-\mathrm{Na}(20)$ & 119(3) \\
\hline $\mathrm{H}(43 \mathrm{~A})-\mathrm{O}(43 \mathrm{~W})-\mathrm{Na}(20)$ & $122(3)$ & $\mathrm{Na}(5)-\mathrm{O}(43 \mathrm{~W})-\mathrm{Na}(20)$ & $93.1(8)$ \\
\hline $\mathrm{H}(44 \mathrm{~B})-\mathrm{O}(44 \mathrm{~W})-\mathrm{H}(44 \mathrm{~A})$ & $110(4)$ & $\mathrm{H}(45 \mathrm{~A})-\mathrm{O}(45 \mathrm{~W})-\mathrm{H}(45 \mathrm{~B})$ & $108(3)$ \\
\hline $\mathrm{H}(46 \mathrm{~A})-\mathrm{O}(46 \mathrm{~W})-\mathrm{H}(46 \mathrm{~B})$ & $107.2(16)$ & $\mathrm{H}(46 \mathrm{~A})-\mathrm{O}(46 \mathrm{~W})-\mathrm{Na}(6)$ & $127.0(13)$ \\
\hline
\end{tabular}




\begin{tabular}{|c|c|c|c|}
\hline $\mathrm{H}(46 \mathrm{~B})-\mathrm{O}(46 \mathrm{~W})-\mathrm{Na}(6)$ & $100.6(12)$ & $\mathrm{H}(47 \mathrm{~A})-\mathrm{O}(47 \mathrm{~W})-\mathrm{H}(47 \mathrm{~B})$ & $122(4)$ \\
\hline $\mathrm{H}(47 \mathrm{~A})-\mathrm{O}(47 \mathrm{~W})-\mathrm{H}(47 \mathrm{C})$ & $103(3)$ & $\mathrm{H}(47 \mathrm{~B})-\mathrm{O}(47 \mathrm{~W})-\mathrm{H}(47 \mathrm{C})$ & $110(5)$ \\
\hline $\mathrm{H}(47 \mathrm{~A})-\mathrm{O}(47 \mathrm{~W})-\mathrm{Na}(16)$ & $107.4(17)$ & $\mathrm{H}(47 \mathrm{~B})-\mathrm{O}(47 \mathrm{~W})-\mathrm{Na}(16)$ & $99(4)$ \\
\hline $\mathrm{H}(47 \mathrm{C})-\mathrm{O}(47 \mathrm{~W})-\mathrm{Na}(16)$ & $115(3)$ & $\mathrm{H}(48 \mathrm{~A})-\mathrm{O}(48 \mathrm{~W})-\mathrm{H}(48 \mathrm{~B})$ & $107.0(17)$ \\
\hline $\mathrm{H}(49 \mathrm{~A})-\mathrm{O}(49 \mathrm{~W})-\mathrm{H}(49 \mathrm{~B})$ & $112(2)$ & $\mathrm{H}(49 \mathrm{~A})-\mathrm{O}(49 \mathrm{~W})-\mathrm{Na}(17)$ & $104.3(17)$ \\
\hline $\mathrm{H}(49 \mathrm{~B})-\mathrm{O}(49 \mathrm{~W})-\mathrm{Na}(17)$ & $118.9(16)$ & $H(50 B)-O(50 W)-H(50 A)$ & $108(2)$ \\
\hline $\mathrm{H}(50 \mathrm{~B})-\mathrm{O}(50 \mathrm{~W})-\mathrm{Na}(17)$ & $97.3(17)$ & $\mathrm{H}(50 \mathrm{~A})-\mathrm{O}(50 \mathrm{~W})-\mathrm{Na}(17)$ & $122.7(18)$ \\
\hline $\mathrm{O}(50 \mathrm{~W})-\mathrm{H}(50 \mathrm{~B})-\mathrm{Na}(17)$ & $61.2(16)$ & $H(51 B)-O(51 W)-H(51 A)$ & $104(3)$ \\
\hline $\mathrm{H}(52 \mathrm{~B})-\mathrm{O}(52 \mathrm{~W})-\mathrm{H}(52 \mathrm{~A})$ & $111(3)$ & $H(53 A)-O(53 W)-H(53 B)$ & $107(3)$ \\
\hline $\mathrm{H}(54 \mathrm{~A})-\mathrm{O}(54 \mathrm{~W})-\mathrm{H}(54 \mathrm{~B})$ & $104(2)$ & $H(55 B)-O(55 W)-H(55 A)$ & $106(3)$ \\
\hline$H(56 B)-O(56 W)-H(56 A)$ & $112(3)$ & $\mathrm{H}(56 \mathrm{~B})-\mathrm{O}(56 \mathrm{~W})-\mathrm{Na}(19)$ & $101(2)$ \\
\hline $\mathrm{H}(56 \mathrm{~A})-\mathrm{O}(56 \mathrm{~W})-\mathrm{Na}(19)$ & $118(3)$ & $\mathrm{O}(56 \mathrm{~W})-\mathrm{H}(56 \mathrm{~B})-\mathrm{Na}(19)$ & $58.1(19)$ \\
\hline $\mathrm{H}(57 \mathrm{~B})-\mathrm{O}(57 \mathrm{~W})-\mathrm{H}(57 \mathrm{~A})$ & $108(4)$ & $\mathrm{H}(58 \mathrm{~A})-\mathrm{O}(58 \mathrm{~W})-\mathrm{H}(58 \mathrm{~B})$ & $115(4)$ \\
\hline $\mathrm{H}(59 \mathrm{C})-\mathrm{O}(59 \mathrm{~W})-\mathrm{O}(59 \mathrm{~V})$ & $76(3)$ & $\mathrm{H}(59 \mathrm{C})-\mathrm{O}(59 \mathrm{~W})-\mathrm{H}(59 \mathrm{~B})$ & $142(3)$ \\
\hline $\mathrm{O}(59 \mathrm{~V})-\mathrm{O}(59 \mathrm{~W})-\mathrm{H}(59 \mathrm{~B})$ & $133(4)$ & $H(59 C)-O(59 W)-H(59 A)$ & $34(3)$ \\
\hline $\mathrm{O}(59 \mathrm{~V})-\mathrm{O}(59 \mathrm{~W})-\mathrm{H}(59 \mathrm{~A})$ & $110(4)$ & $H(59 B)-O(59 W)-H(59 A)$ & $113(4)$ \\
\hline $\mathrm{O}(59 \mathrm{~W})-\mathrm{O}(59 \mathrm{~V})-\mathrm{H}(59 \mathrm{C})$ & $50(4)$ & $\mathrm{O}(59 \mathrm{~W})-\mathrm{O}(59 \mathrm{~V})-\mathrm{H}(59 \mathrm{D})$ & $157(3)$ \\
\hline $\mathrm{H}(59 \mathrm{C})-\mathrm{O}(59 \mathrm{~V})-\mathrm{H}(59 \mathrm{D})$ & $109(4)$ & $\mathrm{O}(59 \mathrm{~W})-\mathrm{O}(59 \mathrm{~V})-\mathrm{H}(59 \mathrm{~A})$ & $39(2)$ \\
\hline $\mathrm{H}(59 \mathrm{C})-\mathrm{O}(59 \mathrm{~V})-\mathrm{H}(59 \mathrm{~A})$ & $11(3)$ & $H(59 D)-O(59 V)-H(59 A)$ & $120(3)$ \\
\hline $\mathrm{O}(59 W)-O(59 V)-K(3)$ & $121.0(19)$ & $H(59 C)-O(59 V)-K(3)$ & $87(3)$ \\
\hline $\mathrm{H}(59 \mathrm{D})-\mathrm{O}(59 \mathrm{~V})-\mathrm{K}(3)$ & $58(2)$ & $H(59 A)-O(59 V)-K(3)$ & $94.9(17)$ \\
\hline $\mathrm{H}(59 \mathrm{C})-\mathrm{H}(59 \mathrm{~A})-\mathrm{O}(59 \mathrm{~W})$ & $51(6)$ & $H(59 C)-H(59 A)-O(59 V)$ & $20(5)$ \\
\hline $\mathrm{O}(59 \mathrm{~W})-\mathrm{H}(59 \mathrm{~A})-\mathrm{O}(59 \mathrm{~V})$ & $31.4(18)$ & $H(59 A)-H(59 C)-O(59 W)$ & $95(7)$ \\
\hline $\mathrm{H}(59 \mathrm{~A})-\mathrm{H}(59 \mathrm{C})-\mathrm{O}(59 \mathrm{~V})$ & $149(8)$ & $\mathrm{O}(59 \mathrm{~W})-\mathrm{H}(59 \mathrm{C})-\mathrm{O}(59 \mathrm{~V})$ & $54.0(18)$ \\
\hline $\mathrm{O}(59 \mathrm{~V})-\mathrm{H}(59 \mathrm{D})-\mathrm{K}(3)$ & $104(2)$ & $H(60 B)-O(60 W)-H(60 A)$ & $117(5)$ \\
\hline $\mathrm{H}(60 \mathrm{~B})-\mathrm{O}(60 \mathrm{~W})-\mathrm{Na}(21)$ & $148(4)$ & $\mathrm{H}(60 \mathrm{~A})-\mathrm{O}(60 \mathrm{~W})-\mathrm{Na}(21)$ & $89(4)$ \\
\hline $\mathrm{H}(60 \mathrm{~B})-\mathrm{O}(60 \mathrm{~W})-\mathrm{Na}(20)$ & $116(3)$ & $\mathrm{H}(60 \mathrm{~A})-\mathrm{O}(60 \mathrm{~W})-\mathrm{Na}(20)$ & $111(3)$ \\
\hline $\mathrm{Na}(21)-\mathrm{O}(60 \mathrm{~W})-\mathrm{Na}(20)$ & $32(2)$ & $\mathrm{O}(60 \mathrm{~W})-\mathrm{H}(60 \mathrm{~A})-\mathrm{Na}(21)$ & $67(3)$ \\
\hline$H(61 B)-O(61 W)-H(61 A)$ & $102(5)$ & $\mathrm{H}(61 \mathrm{~B})-\mathrm{O}(61 \mathrm{~W})-\mathrm{Na}(11) \# 1$ & $116(4)$ \\
\hline $\mathrm{H}(61 \mathrm{~A})-\mathrm{O}(61 \mathrm{~W})-\mathrm{Na}(11) \# 1$ & $98(3)$ & $H(61 B)-O(61 W)-K(3)$ & $80(4)$ \\
\hline$H(61 A)-O(61 W)-K(3)$ & $90(3)$ & $\mathrm{Na}(11) \# 1-\mathrm{O}(61 \mathrm{~W})-\mathrm{K}(3)$ & $160.3(7)$ \\
\hline $\mathrm{H}(62 \mathrm{~B})-\mathrm{O}(62 \mathrm{~W})-\mathrm{H}(62 \mathrm{~A})$ & $60(5)$ & $\mathrm{H}(62 \mathrm{~B})-\mathrm{O}(62 \mathrm{~W})-\mathrm{Na}(3)$ & $123(5)$ \\
\hline $\mathrm{H}(62 \mathrm{~A})-\mathrm{O}(62 \mathrm{~W})-\mathrm{Na}(3)$ & $121(4)$ & $\mathrm{O}(62 \mathrm{~W})-\mathrm{H}(62 \mathrm{~A})-\mathrm{H}(62 \mathrm{~B})$ & $60(3)$ \\
\hline $\mathrm{O}(62 \mathrm{~W})-\mathrm{H}(62 \mathrm{~B})-\mathrm{H}(62 \mathrm{~A})$ & $60(3)$ & $\mathrm{O}(63 \mathrm{~V})-\mathrm{O}(63 \mathrm{~W})-\mathrm{H}(63 \mathrm{~A})$ & $72(3)$ \\
\hline $\mathrm{O}(63 \mathrm{~V})-\mathrm{O}(63 \mathrm{~W})-\mathrm{H}(63 \mathrm{~B})$ & $64(5)$ & $H(63 A)-O(63 W)-H(63 B)$ & $101(4)$ \\
\hline $\mathrm{O}(63 \mathrm{~V})-\mathrm{O}(63 \mathrm{~W})-\mathrm{H}(63 \mathrm{C})$ & $161(5)$ & $H(63 A)-O(63 W)-H(63 C)$ & $113(4)$ \\
\hline $\mathrm{H}(63 \mathrm{~B})-\mathrm{O}(63 \mathrm{~W})-\mathrm{H}(63 \mathrm{C})$ & $96(4)$ & $\mathrm{O}(63 \mathrm{~W})-\mathrm{O}(63 \mathrm{~V})-\mathrm{H}(63 \mathrm{~B})$ & $67(3)$ \\
\hline $\mathrm{O}(63 \mathrm{~W})-\mathrm{O}(63 \mathrm{~V})-\mathrm{H}(63 \mathrm{~A})$ & $62(2)$ & $H(63 B)-O(63 V)-H(63 A)$ & $98(4)$ \\
\hline $\mathrm{O}(63 \mathrm{~W})-\mathrm{H}(63 \mathrm{~A})-\mathrm{O}(63 \mathrm{~V})$ & $46.0(19)$ & $\mathrm{O}(63 \mathrm{~V})-\mathrm{H}(63 \mathrm{~B})-\mathrm{O}(63 \mathrm{~W})$ & $49(3)$ \\
\hline$H(64 D)-O(64 W)-H(64 A)$ & $87(3)$ & $\mathrm{H}(64 \mathrm{D})-\mathrm{O}(64 \mathrm{~W})-\mathrm{H}(64 \mathrm{~B})$ & $59(3)$ \\
\hline $\mathrm{H}(64 \mathrm{~A})-\mathrm{O}(64 \mathrm{~W})-\mathrm{H}(64 \mathrm{~B})$ & 144(3) & $\mathrm{H}(64 \mathrm{D})-\mathrm{O}(64 \mathrm{~W})-\mathrm{H}(64 \mathrm{C})$ & $146(4)$ \\
\hline $\mathrm{H}(64 \mathrm{~A})-\mathrm{O}(64 \mathrm{~W})-\mathrm{H}(64 \mathrm{C})$ & $126(4)$ & $\mathrm{H}(64 \mathrm{~B})-\mathrm{O}(64 \mathrm{~W})-\mathrm{H}(64 \mathrm{C})$ & $87(4)$ \\
\hline $\mathrm{H}(64 \mathrm{D})-\mathrm{O}(64 \mathrm{~W})-\mathrm{Na}(11)$ & $88(2)$ & $\mathrm{H}(64 \mathrm{~A})-\mathrm{O}(64 \mathrm{~W})-\mathrm{Na}(11)$ & $86(2)$ \\
\hline $\mathrm{H}(64 \mathrm{~B})-\mathrm{O}(64 \mathrm{~W})-\mathrm{Na}(11)$ & $104(2)$ & $\mathrm{H}(64 \mathrm{C})-\mathrm{O}(64 \mathrm{~W})-\mathrm{Na}(11)$ & $100(3)$ \\
\hline
\end{tabular}




\begin{tabular}{|c|c|c|c|}
\hline $\mathrm{O}(64 \mathrm{~W})-\mathrm{H}(64 \mathrm{~A})-\mathrm{Na}(11)$ & $72(2)$ & $\mathrm{H}(64 \mathrm{D})-\mathrm{H}(64 \mathrm{~B})-\mathrm{O}(64 \mathrm{~W})$ & $60.4(16)$ \\
\hline$H(64 B)-H(64 D)-O(64 W)$ & $60.3(16)$ & $\mathrm{H}(64 \mathrm{~B})-\mathrm{H}(64 \mathrm{D})-\mathrm{Na}(11)$ & $94(3)$ \\
\hline $\mathrm{O}(64 \mathrm{~W})-\mathrm{H}(64 \mathrm{D})-\mathrm{Na}(11)$ & $69.7(18)$ & $\mathrm{H}(65 \mathrm{~A})-\mathrm{O}(65 \mathrm{~W})-\mathrm{H}(65 \mathrm{~B})$ & $102(6)$ \\
\hline$H(66 A)-O(66 W)-H(66 B)$ & $115(5)$ & $H(67 B)-O(67 W)-H(67 A)$ & $103(4)$ \\
\hline $\mathrm{H}(67 \mathrm{~B})-\mathrm{O}(67 \mathrm{~W})-\mathrm{Na}(6)$ & $120(3)$ & $\mathrm{H}(67 \mathrm{~A})-\mathrm{O}(67 \mathrm{~W})-\mathrm{Na}(6)$ & $107.6(13)$ \\
\hline $\mathrm{H}(68 \mathrm{~A})-\mathrm{O}(68 \mathrm{~W})-\mathrm{H}(68 \mathrm{~B})$ & $108(7)$ & $\mathrm{O}(69 \mathrm{~V})-\mathrm{O}(69 \mathrm{~W})-\mathrm{H}(69 \mathrm{C})$ & $83(5)$ \\
\hline $\mathrm{O}(69 \mathrm{~V})-\mathrm{O}(69 \mathrm{~W})-\mathrm{H}(69 \mathrm{~A})$ & $76(3)$ & $H(69 C)-O(69 W)-H(69 A)$ & $119(5)$ \\
\hline $\mathrm{O}(69 \mathrm{~V})-\mathrm{O}(69 \mathrm{~W})-\mathrm{H}(69 \mathrm{~B})$ & $152(4)$ & $H(69 C)-O(69 W)-H(69 B)$ & $71(5)$ \\
\hline$H(69 A)-O(69 W)-H(69 B)$ & $108(3)$ & $\mathrm{O}(69 \mathrm{~V})-\mathrm{O}(69 \mathrm{~W})-\mathrm{Na}(14)$ & $88(3)$ \\
\hline $\mathrm{H}(69 \mathrm{C})-\mathrm{O}(69 \mathrm{~W})-\mathrm{Na}(14)$ & $128(5)$ & $\mathrm{H}(69 \mathrm{~A})-\mathrm{O}(69 \mathrm{~W})-\mathrm{Na}(14)$ & $108(2)$ \\
\hline $\mathrm{H}(69 \mathrm{~B})-\mathrm{O}(69 \mathrm{~W})-\mathrm{Na}(14)$ & $116(3)$ & $\mathrm{O}(69 \mathrm{~W})-\mathrm{O}(69 \mathrm{~V})-\mathrm{H}(69 \mathrm{~A})$ & $64(3)$ \\
\hline $\mathrm{O}(69 \mathrm{~W})-\mathrm{O}(69 \mathrm{~V})-\mathrm{H}(69 \mathrm{C})$ & $57(5)$ & $H(69 A)-O(69 V)-H(69 C)$ & $100(4)$ \\
\hline $\mathrm{O}(69 \mathrm{~W})-\mathrm{O}(69 \mathrm{~V})-\mathrm{Na}(14)$ & $76(3)$ & $\mathrm{H}(69 \mathrm{~A})-\mathrm{O}(69 \mathrm{~V})-\mathrm{Na}(14)$ & $101(2)$ \\
\hline $\mathrm{H}(69 \mathrm{C})-\mathrm{O}(69 \mathrm{~V})-\mathrm{Na}(14)$ & $113(4)$ & $\mathrm{O}(69 \mathrm{~W})-\mathrm{H}(69 \mathrm{~A})-\mathrm{O}(69 \mathrm{~V})$ & $39.8(17)$ \\
\hline $\mathrm{O}(69 \mathrm{~W})-\mathrm{H}(69 \mathrm{~B})-\mathrm{H}(69 \mathrm{C})$ & $51(4)$ & $\mathrm{O}(69 \mathrm{~W})-\mathrm{H}(69 \mathrm{C})-\mathrm{O}(69 \mathrm{~V})$ & $40(3)$ \\
\hline $\mathrm{O}(69 \mathrm{~W})-\mathrm{H}(69 \mathrm{C})-\mathrm{H}(69 \mathrm{~B})$ & $58(4)$ & $\mathrm{O}(69 \mathrm{~V})-\mathrm{H}(69 \mathrm{C})-\mathrm{H}(69 \mathrm{~B})$ & $97(6)$ \\
\hline $\mathrm{H}(70 \mathrm{~A})-\mathrm{O}(70 \mathrm{~W})-\mathrm{H}(70 \mathrm{~B})$ & 107(9) & $\mathrm{H}(70 \mathrm{~A})-\mathrm{O}(70 \mathrm{~W})-\mathrm{Na}(21)$ & $109(4)$ \\
\hline $\mathrm{H}(70 \mathrm{~B})-\mathrm{O}(70 \mathrm{~W})-\mathrm{Na}(21)$ & 139(9) & $\mathrm{H}(70 \mathrm{~A})-\mathrm{O}(70 \mathrm{~W})-\mathrm{Na}(20)$ & $97(4)$ \\
\hline $\mathrm{H}(70 \mathrm{~B})-\mathrm{O}(70 \mathrm{~W})-\mathrm{Na}(20)$ & $128(9)$ & $\mathrm{Na}(21)-\mathrm{O}(70 \mathrm{~W})-\mathrm{Na}(20)$ & $29(3)$ \\
\hline $\mathrm{O}(70 \mathrm{~W})-\mathrm{H}(70 \mathrm{~A})-\mathrm{Na}(21)$ & $46(3)$ & $\mathrm{O}(70 \mathrm{~W})-\mathrm{H}(70 \mathrm{~B})-\mathrm{Na}(21)$ & $26(6)$ \\
\hline $\mathrm{H}(71 \mathrm{~A})-\mathrm{O}(71 \mathrm{~W})-\mathrm{H}(71 \mathrm{~A}) \# 12$ & 179.997(13) & $\mathrm{H}(71 \mathrm{~A})-\mathrm{O}(71 \mathrm{~W})-\mathrm{H}(71 \mathrm{~B})$ & $77(5)$ \\
\hline $\mathrm{H}(71 \mathrm{~A}) \# 12-\mathrm{O}(71 \mathrm{~W})-\mathrm{H}(71 \mathrm{~B})$ & $103(5)$ & $\mathrm{H}(71 \mathrm{~A})-\mathrm{O}(71 \mathrm{~W})-\mathrm{H}(71 \mathrm{~B}) \# 12$ & $103(5)$ \\
\hline \multicolumn{2}{|c|}{$\mathrm{H}(71 \mathrm{~A}) \# 12-\mathrm{O}(71 \mathrm{~W})-\mathrm{H}(71 \mathrm{~B}) \# 12$ 77(5) } & $\mathrm{H}(71 \mathrm{~B})-\mathrm{O}(71 \mathrm{~W})-\mathrm{H}(71 \mathrm{~B}) \# 12$ & $180.00(2)$ \\
\hline $\mathrm{H}(72 \mathrm{~A})-\mathrm{O}(72 \mathrm{~W})-\mathrm{H}(72 \mathrm{~B})$ & $111(7)$ & $\mathrm{H}(73 \mathrm{~A})-\mathrm{O}(73 \mathrm{~W})-\mathrm{H}(73 \mathrm{~B})$ & $109(8)$ \\
\hline $\mathrm{H}(73 \mathrm{~A})-\mathrm{O}(73 \mathrm{~W})-\mathrm{Na}(20)$ & $122(3)$ & $\mathrm{H}(73 \mathrm{~B})-\mathrm{O}(73 \mathrm{~W})-\mathrm{Na}(20)$ & $101(7)$ \\
\hline $\mathrm{O}(73 \mathrm{~W})-\mathrm{H}(73 \mathrm{~B})-\mathrm{Na}(20)$ & $58(6)$ & $\mathrm{H}(74 \mathrm{~B})-\mathrm{O}(74 \mathrm{~W})-\mathrm{H}(74 \mathrm{C})$ & $106(8)$ \\
\hline $\mathrm{H}(74 \mathrm{~B})-\mathrm{O}(74 \mathrm{~W})-\mathrm{H}(74 \mathrm{~A})$ & $150(6)$ & $\mathrm{H}(74 \mathrm{C})-\mathrm{O}(74 W)-\mathrm{H}(74 \mathrm{~A})$ & $103(7)$ \\
\hline $\mathrm{H}(75 \mathrm{~A})-\mathrm{O}(75 \mathrm{~W})-\mathrm{H}(75 \mathrm{~B})$ & $112(5)$ & $\mathrm{H}(76 \mathrm{~B})-\mathrm{O}(76 \mathrm{~W})-\mathrm{H}(76 \mathrm{~A})$ & $100(3)$ \\
\hline $\mathrm{H}(76 \mathrm{~B})-\mathrm{O}(76 \mathrm{~W})-\mathrm{Na}(7)$ & $102(2)$ & $\mathrm{H}(76 \mathrm{~A})-\mathrm{O}(76 \mathrm{~W})-\mathrm{Na}(7)$ & $109.6(13)$ \\
\hline $\mathrm{H}(76 \mathrm{~B})-\mathrm{O}(76 \mathrm{~W})-\mathrm{K}(1)$ & $173(2)$ & $\mathrm{H}(76 \mathrm{~A})-\mathrm{O}(76 \mathrm{~W})-\mathrm{K}(1)$ & $83.3(15)$ \\
\hline $\mathrm{Na}(7)-\mathrm{O}(76 \mathrm{~W})-\mathrm{K}(1)$ & $82.8(6)$ & $\mathrm{H}(77 \mathrm{~A})-\mathrm{O}(77 \mathrm{~W})-\mathrm{H}(77 \mathrm{~B})$ & $104(3)$ \\
\hline $\mathrm{H}(77 \mathrm{~A})-\mathrm{O}(77 \mathrm{~W})-\mathrm{Na}(17)$ & $106(3)$ & $\mathrm{H}(77 \mathrm{~B})-\mathrm{O}(77 \mathrm{~W})-\mathrm{Na}(17)$ & $107(2)$ \\
\hline $\mathrm{H}(77 \mathrm{~A})-\mathrm{O}(77 \mathrm{~W})-\mathrm{K}(3)$ & 102(3) & $\mathrm{H}(77 \mathrm{~B})-\mathrm{O}(77 \mathrm{~W})-\mathrm{K}(3)$ & $112(2)$ \\
\hline $\mathrm{Na}(17)-\mathrm{O}(77 \mathrm{~W})-\mathrm{K}(3)$ & $123.1(7)$ & & \\
\hline
\end{tabular}

Symmetry transformations used to generate equivalent atoms:

$\# 1-x+2,-y+3,-z-1 \quad \# 2-x+2,-y+3,-z \quad \# 3-x+3,-y+3,-z-1$

\#4 $x, y+1, z \quad \# 5-x+1,-y+4,-z-1 \quad \# 6-x+2,-y+2,-z$

\#7 -x+2,-y+4,-z-1 \#8 $x, y-1, z \quad \# 9-x+3,-y+2,-z$

$\# 10 x+1, y, z \quad \# 11 x-1, y, z \quad \# 12-x+2,-y+1,-z+1$ 